\title{
Visual field progression in patients with open-angle glaucoma
}

Citation for published version (APA):

Ernest, P. J. G. (2014). Visual field progression in patients with open-angle glaucoma. [Doctoral Thesis, Maastricht University]. Universitaire Pers Maastricht. https://doi.org/10.26481/dis.20141212pe

Document status and date:

Published: 01/01/2014

DOI:

10.26481/dis.20141212pe

Document Version:

Publisher's PDF, also known as Version of record

\section{Please check the document version of this publication:}

- A submitted manuscript is the version of the article upon submission and before peer-review. There can be important differences between the submitted version and the official published version of record.

People interested in the research are advised to contact the author for the final version of the publication, or visit the DOI to the publisher's website.

- The final author version and the galley proof are versions of the publication after peer review.

- The final published version features the final layout of the paper including the volume, issue and page numbers.

Link to publication

\footnotetext{
General rights rights.

- You may freely distribute the URL identifying the publication in the public portal. please follow below link for the End User Agreement:

www.umlib.nl/taverne-license

Take down policy

If you believe that this document breaches copyright please contact us at:

repository@maastrichtuniversity.nl

providing details and we will investigate your claim.
}

Copyright and moral rights for the publications made accessible in the public portal are retained by the authors and/or other copyright owners and it is a condition of accessing publications that users recognise and abide by the legal requirements associated with these

- Users may download and print one copy of any publication from the public portal for the purpose of private study or research.

- You may not further distribute the material or use it for any profit-making activity or commercial gain

If the publication is distributed under the terms of Article $25 \mathrm{fa}$ of the Dutch Copyright Act, indicated by the "Taverne" license above, 


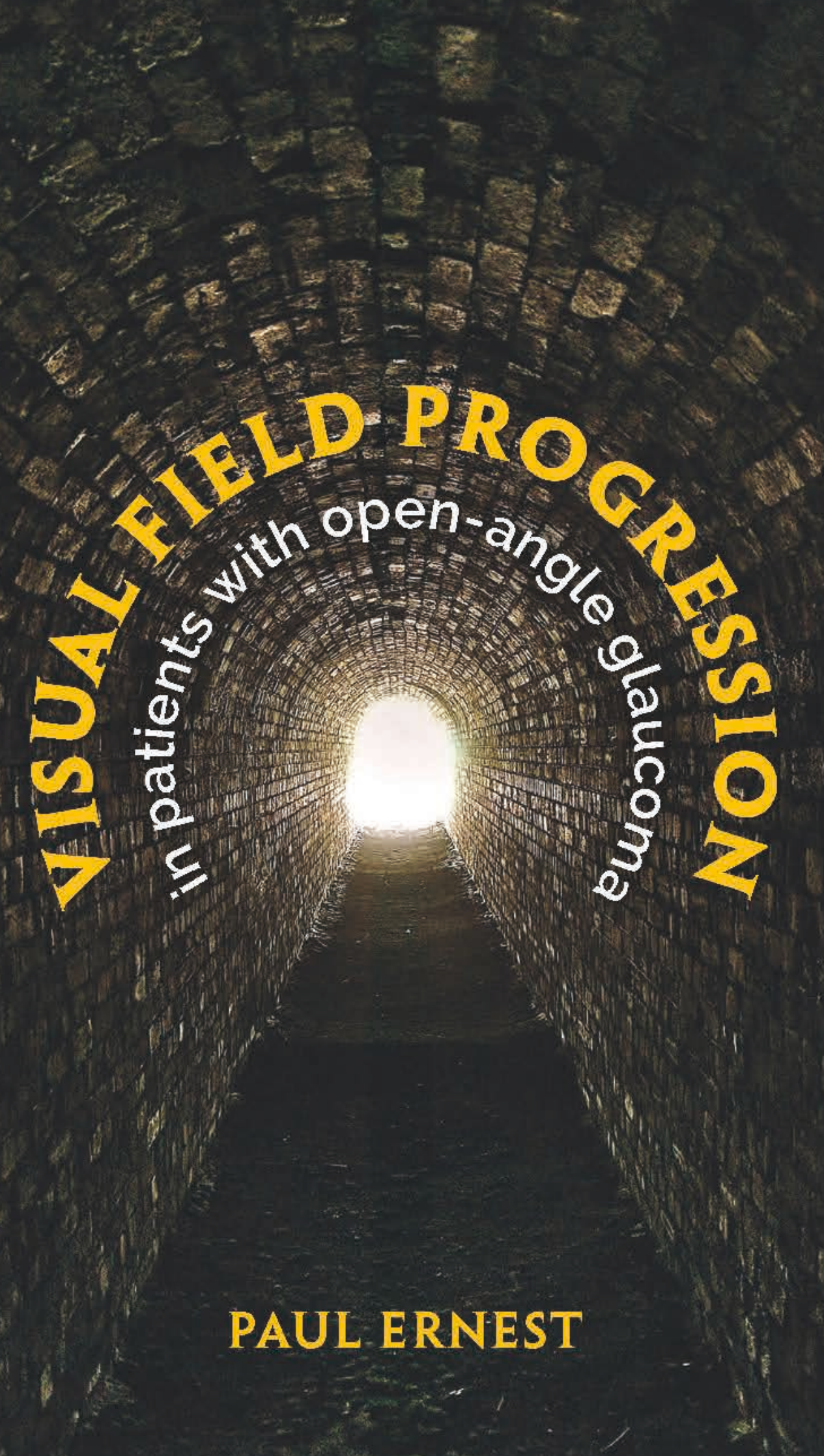


Visual field progression in patients with open-angle glaucoma

Paul J.G. Ernest

ISBN 9789461593603

Cover photograph: Mariusz Kluzniak

Cover and lay-out: Datawyse

Printed by: Datawyse | Universitaire Pers Maastricht

(C) 2014, Paul J.G. Ernest

All rights reserved. No part of this thesis may be reproduced or transmitted in any form or by any means, electronic or mechanical, including photocopying, recording or any information storage or retrieval system, without permission in writing from the author, or, when appropriate, from the publishers of the publications. 


\title{
VISUAL FIELD PROGRESSION IN PATIENTS WITH OPEN-ANGLE GLAUCOMA
}

\author{
PROEFSCHRIFT \\ ter verdediging van de graad van doctor \\ aan de Universiteit Maastricht \\ op gezag van de Rector Magnificus, \\ Prof. dr. L.L.G. Soete, \\ volgens het besluit van het College van Decanen, \\ in het openbaar te verdedigen \\ op vrijdag 12 december 2014 om 14.00 uur \\ door \\ Paul Jakobus Gerardus Ernest \\ Geboren op 24 september 1983 te Halsteren
}

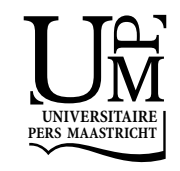




\section{Promotores}

Prof. dr. C.A.B. Webers

Prof. dr. M.H. Prins

\section{Copromotores}

Dr. J.S.A.G. Schouten

Dr. H.J.M. Beckers

\section{Beoordelingscommissie}

Prof. dr. A.E.R.C.H. Boonen (voorzitter)

Prof. dr. J.M.P. Kleijnen

Prof. dr. R.J. van Oostenbrugge

Prof. dr. G.H.M.B. van Rens, VUMC Amsterdam

Dr. L.J. van Rijn, VUMC Amsterdam

The studies in this thesis are partially supported by a grant from "Stichting Oogfonds Nederland" and "Stichting Glaucoomfonds", Utrecht, The Netherlands. 


\section{Table of contents}

Chapter 1: Introduction

Chapter 2: $\quad$ An evidence-based review of prognostic factors for glaucomatous visual field progression Ophthalmology 2013;120:512-519

Chapter 3: $\quad$ Prediction of glaucomatous visual field progression using baseline clinical data Journal of Glaucoma (published ahead-of-print)

Chapter 4: $\quad$ The influence of the assessment method on the incidence of visual field progression in glaucoma:

a network meta-analysis

Acta Ophthalmologica 2012;90:10-19

Chapter 5: $\quad$ The evidence base to select a method for assessing glaucomatous visual field progression Acta Ophthalmologica 2012;90:101-108

Chapter 6: A new strategy to identify the best method for assessing glaucomatous visual field progression Submitted

Chapter 7: $\quad$ Prevalence of end-of-life visual impairment in patients followed for glaucoma Acta Ophthalmologica 2013;91:738-743

Chapter 8: $\quad$ Summarizing discussion

Chapter 9: $\quad$ Valorization addendum

Chapter 10: $\quad$ Samenvatting

Chapter 11: Dankwoord 

CHAPTER 1

Introduction 
The topic of the research presented in this doctoral thesis is glaucomatous visual field progression. This introduction aims to provide a concise context for the subsequent chapters by outlining the background of the research questions that led to this thesis.

\section{Definition of glaucoma}

The term glaucoma refers to a group of diseases that have in common a characteristic optic neuropathy and irreversible loss of visual function. ${ }^{1}$ Glaucomatous optic neuropathy is recognized by progressive optic nerve cupping caused by axonal loss. Patients with glaucoma typically gradually lose their visual field (i.e. peripheral vision) and may lose all vision. Glaucoma is the second leading cause of blindness worldwide. ${ }^{2}$

A major factor in the presumed pathogenesis of glaucoma is intraocular pressure (IOP). Higher IOP has consistently shown to be strongly related with glaucomatous damage and is by far the most important factor and up till now the only proven factor that can be treated. Medical, laser or surgical treatment all aim at lowering IOP. ${ }^{3}$ Although some medications claim to be neuroprotective or have a beneficial effect on perfusion of the optic nerve, there is still not much evidence to support the clinical value of these claims. ${ }^{4,5}$ However, some patients develop glaucomatous damage despite a normal IOP. In this subgroup of normal tension glaucoma (NTG) patients, the disease mechanism is assumed to be mainly caused by additional IOP independent factors. However, lowering intraocular pressure has also shown to slow down disease progression in NTG. ${ }^{6}$ An intraocular pressure above $21 \mathrm{mmHg}$ is generally considered to be outside the range of normal IOP, although this cutoff point is currently seen as highly arbitrary. ${ }^{7}$ Current literature argues that high tension glaucoma and NTG are a continuum of open-angle glaucomas with considerable overlap. ${ }^{8}$

Glaucoma is traditionally classified as open angle or closed angle glaucoma based on the underlying disease mechanism. ${ }^{1} \mathrm{~A}$ high intraocular pressure can be caused by increased production of aqueous humor in the ciliary body of the eye, but more often it is caused by reduced outflow of aqueous humor through the drainage channels of the eye in the angle of the iris and the cornea, the trabecular meshwork. In closed angle glaucoma (ACG), the angle is closed or too narrow leading to a gross obstruction in the outflow of aqueous humor. In contrast, open-angle glaucoma (OAG) is believed to be caused by increased resistance to aqueous outflow in the trabecular meshwork. This thesis is mainly about OAG. The mean worldwide prevalence of $O A G$ is estimated at $2 \%$ in people over 40 years in $2010 .^{9}$ OAG is the most prevalent type of glaucoma in Europe 
with an estimated prevalence of $1.97 \%$ in 2010. In contrast, the estimated European prevalence for ACG in 2010 is $0.25 \%{ }^{9}$

Glaucoma can also be classified as primary or secondary depending on the presence or absence of ocular or systemic disorders contributing to the pressure rise. ${ }^{1}$ Within OAG, primary OAG (POAG) and NTG are the most common manifestations of the disease. Pseudoexfoliation glaucoma (PXG) and pigmentary glaucoma (PG) are examples of secondary OAG that are commonly studied together with POAG, as in this thesis. The secondary condition of PXG is exfoliation syndrome, in which an abnormal fibrillogranular protein (exfoliation material) is produced in the eye. The trabecular outflow of the eye is reduced owing to the accumulation of the exfoliation material in the trabecular meshwork. ${ }^{10}$ In PG, the trabecular meshwork is impeded by melanin granules originated from the iris. ${ }^{11}$ There are several other forms of secondary OAG such as uveitic glaucoma, glaucoma due to corticosteroid treatment, and glaucoma caused by increased episcleral venous pressure. ${ }^{12}$ These subgroups are generally studied separately and are not covered in this thesis.

\section{Treatment of glaucoma}

There is currently no medical intervention that can repair damaged retinal nerve fibers in a glaucomatous eye, so there is no cure for glaucoma. Treatment of glaucoma is directed at lowering IOP to slow down disease progression. The first-line treatment to lower IOP is medication that reduces production of aqueous humor in the ciliary body and/or increases outflow of aqueous humor through the trabecular meshwork or uveoscleral outflow. The second treatment modality is a laser procedure to open up the trabecular meshwork. This could also be the first-line treatment for patients when medications are not available or when the patient's adherence to prescribed medication is low. Glaucoma surgery is the third and most invasive treatment to lower IOP. Surgery aims to improve outflow of aqueous humor through an alternative drainage route like the creation of a filtering bleb or implantation of a drainage device.

\section{Visual function in glaucoma}

Testing in glaucoma can be divided into diagnosis of disease and monitoring of already diagnosed glaucoma patients. This thesis is about monitoring of glaucoma patients to determine whether glaucomatous damage is worsening. Worsening of glaucomatous damage is termed "disease progression". 
Monitoring of patients with glaucoma is based on identification of structural and functional changes in the eye. Measurement of structural changes mainly consists of evaluation of the optic disc with use of a slit lamp or scanning laser ophthalmoscopy, and evaluation of the retinal nerve fiber layer with use of scanning laser polarimetry or optical coherence tomography. Visual field testing (i.e. perimetry) is the most common functional measurement in glaucoma. It should be performed in all patients with glaucoma according to current guidelines. ${ }^{12,13}$ More than $99 \%$ of eye departments in the UK use some form of perimetry. ${ }^{14}$ Because visual field loss and relevant patient outcomes, like visual disability and quality of life, have shown to be related, the research in this thesis is focused on visual field testing. ${ }^{15-19}$

The normal visual field extends approximately $50^{\circ}$ superiorly, $60^{\circ}$ nasally, $70^{\circ}$ inferiorly and $90^{\circ}$ temporally. Visual field function is generally assessed by standardized automated perimetry (SAP), which refers to static computerized threshold perimetry of the central $30^{\circ}$ or $24^{\circ}$ visual field performed with ordinary white stimuli on a white background. ${ }^{12}$ During the test, a stationary test object is increased in intensity from below threshold until perceived by the patient. ${ }^{1}$ This is repeated at different visual field locations. Another type of visual field examinations is manual Goldmann kinetic perimetry. This older method is still useful, e.g. for patients that are unable to perform SAP and for neuro-ophthalmologic patients. In kinetic perimetry, a test object of fixed intensity is moved along several meridians toward fixation. Points where the object is first perceived are plotted in a circle. ${ }^{1}$

Different perimeters are available for the performance of SAP. In this thesis, we focus on the Humphrey Field Analyzer (HFA) because a majority of studies in the literature used this perimeter. The frequency of its use has even more increased to $77 \%$ of the studies published since 2000 (see Chapter 3). An example of a single visual field printout of a glaucoma patient's right eye from the HFA is shown in Figure 1.

One of the concerns in modern perimetry is the significant intertest variability in glaucoma patients, which makes it difficult to determine with statistical confidence whether glaucomatous damage is worsening or not. ${ }^{20}$ Furthermore, it is essential that each SAP test is a high quality measurement of the patient's visual function at that point in time. ${ }^{13}$ An important factor to obtain a high quality measurement is the suitability of the patient for visual field testing. For example, the patient should be physically able to sit at the perimeter and should be sufficiently intellectually competent to undertake the test. The patient should also have any central visual function to enable adequate fixation during the test. Other important factors are appropriate near refractive correction and adequate supervision by a technician during the test. ${ }^{13}$ 


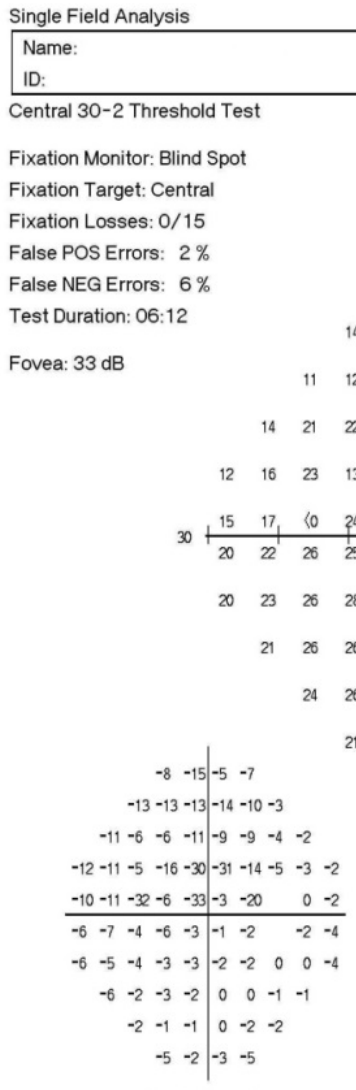

Total Deviation

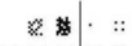

I I I

格四回 :

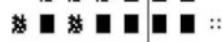

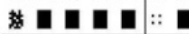

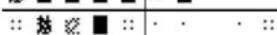

:: 米. : :

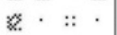

.. $\quad::<5 \%$

\&) $<2 \%$

娄 $<1 \%$

- $<0.5 \%$
Stimulus: III, White

Background: 31.5 ASB

Strategy: SITA-Fast
Pupil Diameter: $4.3 \mathrm{~mm}$

Visual Acuity: 0.8

RX: +0.50 DS DC X
Date: 25-02-2003

Time: 14:01

Age: 87

Eye: Right

$\mathrm{DOB}$ :

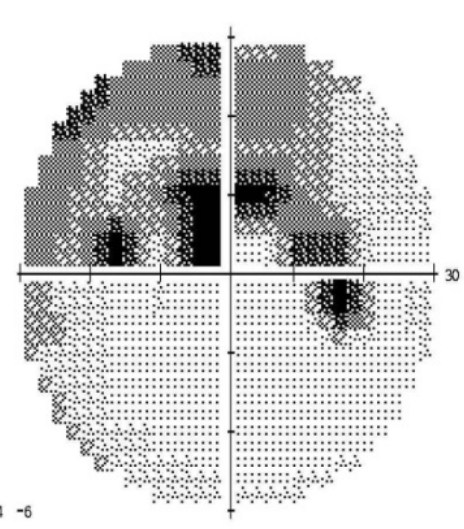

$7|-14|-4 \quad-6$

$-12-12-12-13-9-2$

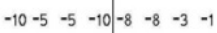

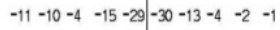

\begin{tabular}{lllllllll}
-9 & -10 & -31 & -5 & -32 & -2 & -19 & 1 & -1 \\
\hline-5 & -6 & -3 & -5 & -2 & 0 & -1 & -1 & -3
\end{tabular}

GHT

\begin{tabular}{lllll|lllll}
-5 & -4 & -3 & -2 & -2 & -1 & -1 & 1 & 1 & -3
\end{tabular}

\begin{tabular}{llll|llll}
-5 & -1 & -2 & -1 & 1 & 1 & 0 & 0
\end{tabular}

Outside normal limits

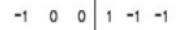

\begin{tabular}{ll|ll}
-4 & -1 & -2 & -4
\end{tabular}

VFI $\quad 75 \%$

MD $\quad-7.41 \mathrm{~dB} P<0.5 \%$

PSD $\quad 9.44 d B P<0.5 \%$

Pattern Deviation

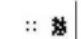

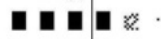

\$: :

\%.

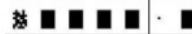

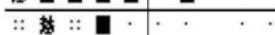

:... . . . . .

: . . . . . .

..

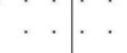

Figure 1: A single visual field printout of a glaucoma patient's right eye from the Humphrey Field Analyzer. 
The visual function as determined by SAP is commonly expressed as a mean value of different points in the visual field, a visual field index. Two frequently used visual field indices from the HFA are the Mean Deviation (MD) value and the Visual field index (VFI). Compared with the MD value, the VFI is more concentrated on central areas of the visual field. ${ }^{21}$ The MD is expressed on a decibel scale, where 0 decibel represents a normal visual field and -30 decibels an almost extinguished visual field. The VFI is expressed as a percentage, where 100\% represents a normal visual field and $0 \%$ represents a perimetrically blind eye.

\section{Progression of glaucoma}

The goal of glaucoma treatment is to maintain the patient's visual function and related quality of life, at a sustainable cost. ${ }^{12}$ Because glaucoma patients have irreversible visual field loss by definition, the goal is thus to prevent progression to a more advanced disease stage which would cause a further loss of quality of life. It is therefore important to regularly and reliably monitor glaucoma patients to detect early deterioration of the visual field. The ability to differentiate progressing patients from patients with stable disease would direct more intensive treatment to those who are at the highest risk for visual disability. ${ }^{22}$ Beside the presence of glaucomatous progression, the individual probability that glaucomatous damage will affect a patient's quality of life is also dependent on the patient's age and the current stage of disease. ${ }^{23}$ On the other hand, a correct method to assess progression will reduce side effects of glaucoma treatment for patients with stable disease. However, the criteria by which progression should be determined are subject of ongoing debate. ${ }^{21,24-29}$

The frequency of visual field testing has also been a subject of debate in the last years. Recently, experts have reached consensus about some important basic principles of performing visual field examinations. ${ }^{13}$ First, a learning effect may occur during the patient's initial field examination. ${ }^{30}$ When an obvious learning effect is present, the first visual field examination should be replaced by a new visual field. There is consensus to perform two baseline visual fields in the first six months of follow-up. ${ }^{13}$ Performing six visual fields in the first two years enables the clinician to rule out rapid visual field progression. ${ }^{31}$ Because time and money constraints, timing of follow-up visual fields could be made dependent on the results of previous visual field examinations. As soon as progression is suspected to occur, the frequency of visual field examinations is increased to confirm or refute the progression. ${ }^{32}$ Following this principle of adaptive testing, the perimetric frequency can be reduced to typically one test per year in patients at low or medium risk to visual field progression. 


\section{Risk factors for glaucoma}

Risk factors can be classified into etiologic factors, prognostic factors, and diagnostic factors. ${ }^{33}$ Etiologic factors need to have a causal relation with a disease. Prognostic factors and diagnostic factors are merely used to predict current disease (i.e. diagnostic factors) or to predict disease in the future (i.e. prognostic factors). Prognostic and diagnostic factors do not need to have a causal relation with the disease, but they should be practical so that they can be used by clinicians to predict disease. Prognostic factors for OAG can be subdivided into prognostic factors for the incidence of OAG, for progression of OAG, and risk factors for treatment response in OAG patients. ${ }^{34}$

Although glaucoma is a leading cause of blindness, most patients with OAG will retain some vision for their entire lives. The ability to differentiate rapidly progressing patients from slowly progressing patients would greatly help clinicians in daily clinical practice to administer the best required treatment. Ultimately, clinical prediction models could be developed to further facilitate the aggregation of knowledge on several prognostic factors for clinicians. ${ }^{35,36}$ We are aware of just a few predictive models for visual field progression in glaucoma that have been published. ${ }^{37,38}$

\section{Research in this thesis}

Following the importance of measuring glaucomatous visual field progression to reach the ultimate goal of glaucoma treatment (i.e. the maintenance of a patient's quality of life), the aim of the research presented in this doctoral thesis was to further investigate the phenomenon of glaucomatous visual field progression. We investigated how accurately it can be predicted and gave an overview of the most important prognostic factors for glaucomatous visual field progression. We investigated the way progression can be determined and how choosing a different method to assess progression will influence study results. Furthermore, the literature was summarized and empirical research was undertaken to give an advice on the best method to assess progression. Finally, we clarified the incidence of end-of-life visual disability and its possible causes. 


\section{Research questions}

From the previous knowledge about glaucoma and the measurement of glaucomatous progression we formulated the following research questions:

1. What are the prognostic factors for glaucomatous visual field progression? (Chapter 2)

2. Can visual field progression be accurately predicted with the use of available prognostic factors? (Chapter 3)

3. To what extent does the method to assess progression influence the incidence of progression? (Chapter 4)

4. To what extent have currently available assessment methods been validated? (Chapter 5)

5. What is the best method to assess glaucomatous visual field progression taking into account the relation with prognostic factors? (Chapter 6 )

6. What is the prevalence of end-of-life visual disability in treated glaucoma patients? (Chapter 7) 


\section{References}

1. American Academy of Ophthalmology. Basic and clinical science course 2010-2011 section 10: Glaucoma. San Francisco: American Academy of Ophthalmology, 2010.

2. Resnikoff S, Pascolini D, Etya'ale D, Kocur I, Pararajasegaram R, Pokharel GP, et al. Global data on visual impairment in the year 2002. Bull World Health Organ 2004;82:844-51.

3. Bahrami H. Causal inference in primary open angle glaucoma: specific discussion on intraocular pressure. Ophthalmic Epidemiol 2006;13:283-9.

4. Sena DF, Lindsley K. Neuroprotection for treatment of glaucoma in adults. Cochrane Database Syst Rev 2013;2:Cd006539.

5. Caprioli J, Coleman AL. Blood pressure, perfusion pressure, and glaucoma. Am J Ophthalmol 2010;149:704-12.

6. Anderson DR, Drance SM, Schulzer M. Comparison of glaucomatous progression between untreated patients with normal-tension glaucoma and patients with therapeutically reduced intraocular pressures. Am J Ophthalmol 1998;126:487.

7. Sommer A. Ocular hypertension and normal-tension glaucoma: time for banishment and burial. Arch Ophthalmol 2011;129:785-7.

8. Shields MB. Normal-tension glaucoma: is it different from primary open-angle glaucoma? Curr Opin Ophthalmol 2008;19:85-8.

9. Quigley HA, Broman AT. The number of people with glaucoma worldwide in 2010 and 2020. Br J Ophthalmol 2006;90:262-7.

10. Ritch R. Exfoliation syndrome. Curr Opin Ophthalmol 2001;12:124-30.

11. Ritch R. Pigment dispersion syndrome. Am J Ophthalmol 1998;126:442-5.

12. European Glaucoma Society. Terminology and Guidelines for Glaucoma (3rd ed.). Savona, Italy: Dogma, 2008.

13. World Glaucoma Association. Progression of Glaucoma, Consensus Series 8. Amsterdam: Kugler Publications, 2011.

14. Gordon Bennett PS, loannidis AS, Papageorgiou K, Andreou PS. A survey of investigations used for the management of glaucoma in hospital service in the United Kingdom. Eye (Lond) 2008;22:1410-8.

15. Viswanathan AC, McNaught Al, Poinoosawmy D, Fontana L, Crabb DP, Fitzke FW, et al. Severity and stability of glaucoma: patient perception compared with objective measurement. Arch Ophthalmol 1999;117:450-4.

16. Gutierrez P, Wilson MR, Johnson C, Gordon M, Cioffi GA, Ritch R, et al. Influence of glaucomatous visual field loss on health-related quality of life. Arch Ophthalmol 1997;115:777-84.

17. Kobelt G, Jonsson B, Bergstrom A, Chen E, Linden C, Alm A. Cost-effectiveness analysis in glaucoma: what drives utility? Results from a pilot study in Sweden. Acta Ophthalmol Scand 2006;84:363-71.

18. McKean Cowdin R, Wang Y, Wu J, Azen SP, Varma R. Impact of visual field loss on health-related quality of life in glaucoma: the Los Angeles Latino Eye Study. Ophthalmology 2008;115:941-8.e1.

19. van Gestel A, Webers CA, Beckers HJ, van Dongen MC, Severens JL, Hendrikse F, et al. The relationship between visual field loss in glaucoma and health-related quality-of-life. Eye 2010;24:1759-69.

20. Heijl A, Lindgren A, Lindgren G. Test-retest variability in glaucomatous visual fields. Am J Ophthalmol 1989;108:130-5.

21. Bengtsson B, Heijl A. A visual field index for calculation of glaucoma rate of progression. Am J Ophthalmol 2008;145:343-53.

22. Caprioli J. The importance of rates in glaucoma. Am J Ophthalmol 2008;145:191-2.

23. Rossetti L, Goni F, Denis P, Bengtsson B, Martinez A, Heijl A. Focusing on glaucoma progression and the clinical importance of progression rate measurement: a review. Eye 2010;24 Suppl 1:S1-7. 
24. Katz J, Congdon N, Friedman DS. Methodological variations in estimating apparent progressive visual field loss in clinical trials of glaucoma treatment. Arch Ophthalmol 1999;117:1137-42.

25. Lee AC, Sample PA, Blumenthal EZ, Berry C, Zangwill L, Weinreb RN. Infrequent confirmation of visual field progression. Ophthalmology 2002;109:1059.

26. Mayama C, Araie M, Suzuki Y, Ishida K, Yamamoto T, Kitazawa Y, et al. Statistical evaluation of the diagnostic accuracy of methods used to determine the progression of visual field defects in glaucoma. Ophthalmology 2004;111:2117.

27. Nouri Mahdavi K, Brigatti L, Weitzman M, Caprioli J. Comparison of methods to detect visual field progression in glaucoma. Ophthalmology 1997;104:1228.

28. Vesti E, Johnson CA, Chauhan BC. Comparison of different methods for detecting glaucomatous visual field progression. Invest Ophthalmol Vis Sci 2003;44:3873.

29. Heijl A, Bengtsson B, Chauhan BC, Lieberman MF, Cunliffe I, Hyman L, et al. A comparison of visual field progression criteria of 3 major glaucoma trials in early manifest glaucoma trial patients. Ophthalmology 2008;115:1557-65.

30. Heijl A, Bengtsson B. The effect of perimetric experience in patients with glaucoma. Arch Ophthalmol 1996;114:19-22.

31. Chauhan BC, Garway Heath DF, Goni FJ, Rossetti L, Bengtsson B, Viswanathan AC, et al. Practical recommendations for measuring rates of visual field change in glaucoma. Br J Ophthalmol 2008;92:56973.

32. Jansonius NM. Towards an optimal perimetric strategy for progression detection in glaucoma: from fixedspace to adaptive inter-test intervals. Graefes Arch Clin Exp Ophthalmol 2006;244:390-3.

33. Grobbee DE, Hoes AW. Clinical epidemiology : principles, methods, and applications for clinical research. Sudbury, Mass.: Jones and Bartlett Publishers, 2009.

34. Boland MV, Quigley HA, Boland MV, Quigley HA. Risk factors and open-angle glaucoma: classification and application. J Glaucoma 2007;16:406-18.

35. Moons KG, Royston P, Vergouwe Y, Grobbee DE, Altman DG. Prognosis and prognostic research: what, why, and how? BMJ 2009;338:b375.

36. Medeiros FA, Weinreb RN. Predictive models to estimate the risk of glaucoma development and progression. Prog Brain Res 2008;173:15-24.

37. Nouri Mahdavi K, Hoffman D, Gaasterland D, Caprioli J. Prediction of visual field progression in glaucoma. Invest Ophthalmol Vis Sci 2004;45:4346-51.

38. De Moraes CG, Sehi M, Greenfield DS, Chung YS, Ritch R, Liebmann JM. A validated risk calculator to assess risk and rate of visual field progression in treated glaucoma patients. Invest Ophthalmol Vis Sci 2012;53:2702-7. 


\title{
CHAPTER 2
}

An evidence-based review of prognostic factors for glaucomatous visual field progression

\author{
Authors \\ Paul J.G. Ernest \\ Jan S.A.G. Schouten \\ Henny J.M. Beckers \\ Fred Hendrikse \\ Martin H. Prins \\ Carroll A.B. Webers
}

Ophthalmology 2013;120:512-519 


\section{ABSTRACT}

Topic: An examination of which prognostic factors are associated with glaucomatous visual field progression.

Clinical Relevance: Knowledge of prognostic factors helps clinicians to select patients at risk of glaucomatous visual field progression and intensify their treatment.

Methods/Literature Reviewed: Consulting relevant databases, we identified 2733 articles published up to September 2010, of which 85 articles investigating prognostic factors for visual field progression in patients with open-angle glaucoma were eligible. We summarized results for each factor in tables, noting the direction of the association between the prognostic factor and progression, and the accompanying $P$ value. Four authors, working blind to the factors, independently judged the extent to which a prognostic factor was associated with glaucomatous visual field progression. If there were different associations for normal-tension glaucoma (NTG) studies, they were judged separately. Consensus was reached at group meetings.

Results: One hundred and three different prognostic factors were investigated in 85 articles. The following factors were clearly associated with glaucomatous visual field progression: age, disc hemorrhages (for NTG), baseline visual field loss, baseline intraocular pressure, and exfoliation syndrome. An association was unlikely for family history of glaucoma, atherosclerosis, systemic hypertension, visual acuity, gender (for NTG), systolic blood pressure, myopic refractive error (for NTG), and Raynaud's phenomenon.

Conclusion: The factors we found clearly associated with progression could be used in clinical practice and for developing clinical prediction models. For many other factors, further research is necessary. 
Open-angle glaucoma (OAG) is a chronic, progressive optic neuropathy with a characteristic appearance of the optic nerve and associated visual field defects. ${ }^{1}$ Even in treated OAG patients, glaucoma may lead to blindness. ${ }^{2,3}$ Hence, knowledge of prognostic factors is important to identify patients at risk of rapid progression, who need more intensive treatment to prevent blindness.

Prognostic factors can be used to create prognostic profiles for individual patients. For example, OAG patients with exfoliation syndrome frequently show higher rates of progression compared to OAG patients without exfoliation syndrome. ${ }^{4} \mathrm{~A}$ likely prognosis for a patient could guide subsequent medical actions, such as intensity of treatment and frequency of follow-up as well as the decision to refrain from certain interventions (e.g., glaucoma surgery). Therefore, a patient's prognosis is of key clinical importance. ${ }^{5}$

Earlier reviews have addressed the issue of prognostic factors for glaucomatous visual field progression. ${ }^{6-8}$ However, the evidence of the association between distinct prognostic factors and visual field progression has not been evaluated systematically. The outcomes of many studies on prognostic factors have been variable, sometimes contradictory, and although current literature argues that both OAG with higher and lower intraocular pressures (IOPs) represent one disease continuum, many studies on prognostic factors judged glaucoma patients with IOP in a normal range separately, making results difficult to interpret.

The purpose of the present review is to systematically study to what extent various prognostic factors have been investigated and which factors are most consistently associated with glaucomatous visual field progression.

\section{Sources and methods of literature search}

\section{Search strategy}

In September 2010, we conducted a systematic search in PubMed, EMBASE, and all databases and registers of The Cochrane Library, using these keywords: (glaucoma*) AND (prognos* OR predict* OR progress* OR longitudinal OR cohort OR follow-up) AND (perimetr* OR visual field* OR HFA (Humphrey Field Analyzer) OR Octopus OR Humphrey (not in author)). The search was limited to articles in English, Dutch, French, or German. Articles in press were also selected. In total, we identified 2733 articles. 


\section{Selection process}

We applied the same broad exclusion criteria as in two previous systematic reviews on visual field progression in glaucoma (Figure 1). ${ }^{9} 10$ Four additional exclusion criteria were applied to find eligible studies on prognostic factors for glaucomatous visual field progression (Figure 1). We were only interested in baseline prognostic factors because they can be used to predict progression. "Baseline" is defined as "present in a treated or untreated glaucoma patient before the follow-up period in which visual field progression could occur." Prognostic factors in studies were included only if an association was statistically tested. Studies that investigated only interaction terms or clinical interventions were excluded. Furthermore, we included only studies that assessed visual field progression quantitatively (i.e., with numeric units for defining progression) and excluded studies that used qualitative methods (i.e., subjective descriptions of progression; e.g., "appearance of a nasal step") and those that did not define the method used to assess progression. All selected studies had to differentiate between eyes with or without baseline visual field loss, because risk factors for glaucoma conversion might differ from prognostic factors for glaucoma progression. Finally, at least $85 \%$ of patients analyzed in a study had to be diagnosed with OAG. Of the 2733 identified articles, 85 fulfilled the predefined selection criteria (Figure 1). We compared the search and selection process of our review with the article selection of a review on methodological quality of studies on prognostic factors. ${ }^{11}$

\section{Study design and overlap}

Studies were included in the review irrespective of their design because all designs were essentially suited to investigating prognostic factors. We included 62 case series, 21 cohort studies, and two case-control studies as defined according to Ophthalmology's study design scheme (Table 1). ${ }^{12}$ We classified all studies in independent study clusters. Studies with populations likely to overlap with populations from another selected study were classified in the same cluster. We determined the possibility of overlap between studies using information on the patient-recruiting hospitals, author names, trial names, references to other studies, and period of recruitment. The 85 articles contained 45 independent study clusters (Table 1).

\section{Study quality}

To determine study quality we used a checklist developed for quality assessment of prognosis studies recommended by the National Institute for Health and Clinical Excellence (NICE). ${ }^{13}$ This checklist contains six items on the representativeness of the population, loss to follow-up, measurement of prognostic factors, outcome measurement, correction for potential confounders, and suitability of the statistical analysis. Table 2 summarizes the quality of included studies. In total, we found two studies (2\%) with 
"yes" for all six items, 42 studies (49\%) for five items, 27 studies (32\%) for four items, six studies (7\%) for three items, five studies (6\%) for two items, and three studies (4\%) with "yes" for one item.

\section{Data analysis and interpretation}

Even studies of similar design were difficult to compare, mainly due to the many different methods used to assess progression, the use of many different prognostic factors, differences in study methodology, the high number of studies with correlated results, and adjustment for different prognostic factors in the multivariable analysis. For example, different methods of assessing progression may give varying incidences of visual field progression, ranging from $2 \%$ to $62 \%$ in six years due solely to the method. ${ }^{9}$ Moreover, the choice of the method to assess progression and the statistical approach used to test the association may influence the strength of the association between a prognostic factor and progression. ${ }^{14}$ Given that the group of studies was insufficiently homogeneous to provide a meaningful summary, we did not conduct a formal metaanalysis. ${ }^{15}$

Instead, we used a semi-quantitative approach to summarize the results from multiple studies, as we had done in a previous review. ${ }^{16}$ First, we summarized the evidence of each prognostic factor in an evidence table for the univariate results and when present the multivariable results corrected for the effects of other variables. The tables classified each study of a prognostic factor according to the direction of the association between the factor and glaucomatous visual field progression (i.e., More progression, No relation, or Less progression in the presence of a prognostic factor), and the reported statistical significance of the association (i.e., statistically significant or nonsignificant, using a $P$ value $\leq 0.05)$. We extracted one conclusion per study for each prognostic factor. A study was classified between statistically significant and nonsignificant when one analysis resulted in a significant association and the other analysis of the same study resulted in a non-significant association. Table 3, shows a sample evidence table. We indicated separately those studies comprising a majority ( $>50 \%)$ of NTG patients (e.g., studies 5 and 9 in Table 3). We used study-specific definitions to define patients with NTG and found 20 studies (24\%) with mainly NTG patients (Table 1). Furthermore, we indicated all clusters of studies with correlated results (e.g., studies $1 a$ and $1 b$ in Table 3).

Tables with univariate and multivariable results were used to determine the extent to which a presumed prognostic factor was associated with glaucomatous visual field progression. Four investigators (JSS, HJB, CAW, and PJE) independently judged whether the factor was not associated, possibly associated, probably associated, or definitely 
associated with visual field progression. All investigators were blinded for the factor under investigation and the study that assessed it. When assessing associations, they took into account the total number of studies that tested an association, any clustering in the results, and the total number of patients investigated in the studies. Higher numbers of studies, clusters, and patients were weighted more heavily than lower numbers. Whenever available, multivariable results were weighted more heavily than univariate results, because these are corrected for the confounding effects of other factors. Results from the 20 NTG studies were initially judged separately. In group meetings, the four investigators reached consensus on all differences in individual judgments. When the investigators noticed a considerable amount of heterogeneity in the study results, this was also recorded. The entire assessment process was repeated one month later to judge the consistency of the assessments. We used the weighted kappa statistic $(k)$ with quadratic weights to indicate the strength of agreement between the categories assigned in both consensus rounds. ${ }^{17,18}$

Finally, we rated the clinical importance of a prognostic factor according to Ophthalmology's guidelines. ${ }^{12}$ If a prognostic factor was prevalent, easy to assess, and judged clearly (i.e., definitely or probably) associated with glaucomatous visual field progression, we rated it as very important, level A. If a factor was judged clearly associated with progression but not prevalent or easy to assess, we rated it as moderately important, level B. Factors with a weaker (i.e., possible) association and in need of more research to clarify clinical relevance were rated as possibly relevant, level $\mathrm{C}$. The body of evidence of a prognostic factor was rated as strong (level I), substantial (level II), or weak (level III), analogous to our judgments as "definitely," "probably," or "possibly" associated with visual field progression. Prognostic factors that we judged as "not associated" with visual field progression were rated as unlikely prognostic factors if they had been investigated in ten or more studies. 
2733 Potentially relevant articles identified and screened for retrieval

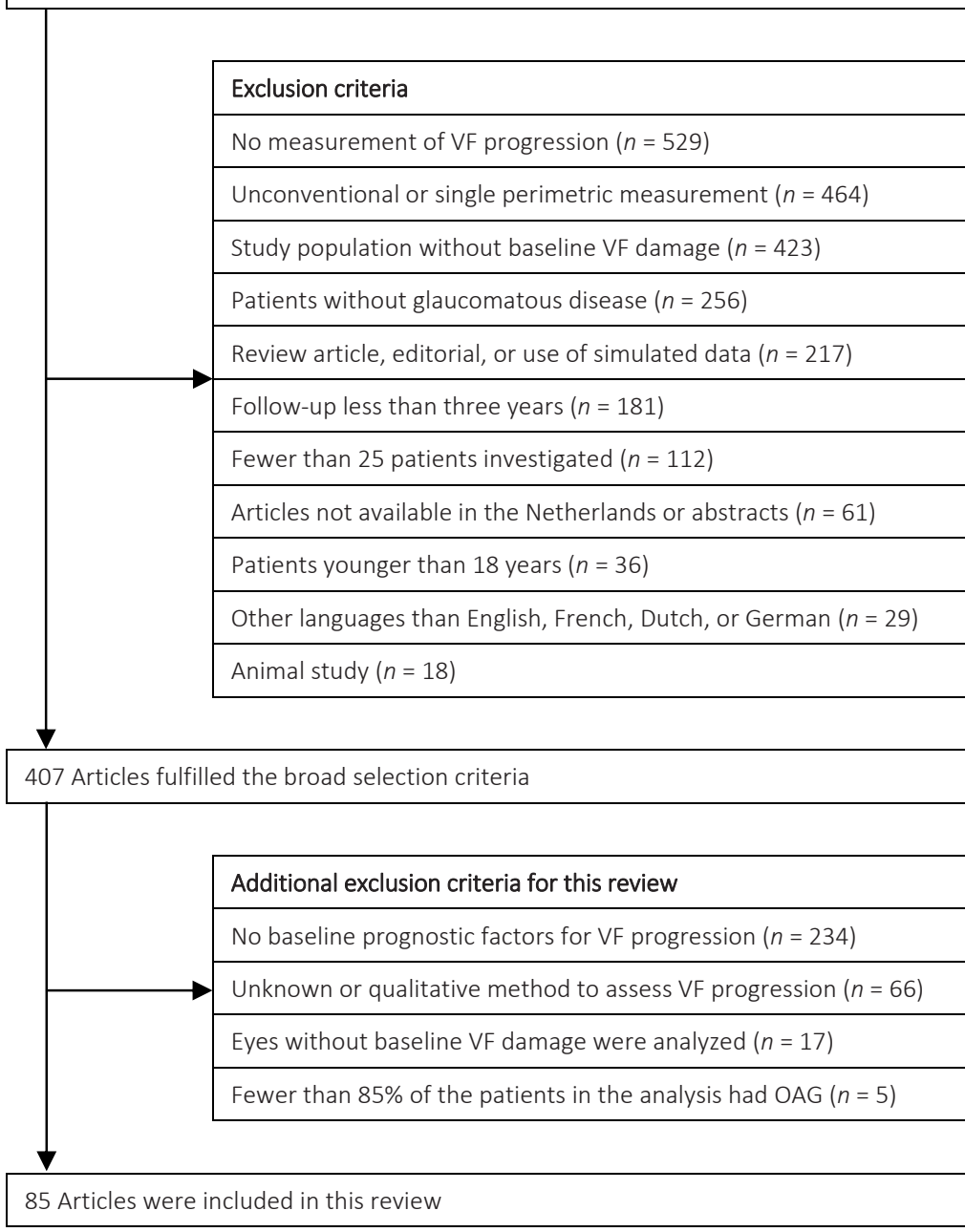

Figure 1: Flow chart of article selection process $\mathrm{VF}=$ visual field, $\mathrm{OAG}=$ open-angle glaucoma 
Table 1: Characteristics of the 85 included studies

\begin{tabular}{|c|c|c|c|c|c|}
\hline \multirow{2}{*}{$\begin{array}{l}\text { Cluster } \\
\text { number }\end{array}$} & \multirow{2}{*}{$\begin{array}{l}\text { Publication } \\
\text { year }\end{array}$} & \multirow[t]{2}{*}{ Source of study population } & \multicolumn{3}{|c|}{ Study references } \\
\hline & & & $\begin{array}{l}\text { Case } \\
\text { series }\end{array}$ & $\begin{array}{l}\text { Cohort } \\
\text { studies }\end{array}$ & $\begin{array}{l}\text { CC } \\
\text { studies }\end{array}$ \\
\hline 1 & 1977 & $\begin{array}{l}\text { University of British Columbia and Vancouver General } \\
\text { Hospital, Vancouver, BC, Canada; University of Utah, } \\
\text { Salt Lake City, UT, USA }\end{array}$ & 1 & & \\
\hline 2 & 1982-1990 & King's College Hospital Glaucoma Centre, London, UK & 2,3 & & \\
\hline 3 & 1984 & University of Cologne, Cologne, Germany & 4 & & \\
\hline 4 & 1986-1992 & $\begin{array}{l}\text { St Paul's Hospital and University of British Columbia } \\
\text { Eye Clinic, Vancouver, BC, Canada }\end{array}$ & 5,6 & & \\
\hline 5 & 1988-1993 & University Eye Clinic Wurzburg, Wurzburg, Germany & 7,8 & & \\
\hline 6 & 1989 & I'Hospital Saint-Joseph, Paris, France & 9 & & \\
\hline 7 & 1989 & Wills Eye Hospital, Philadelphia, PA, USA & $10^{*}$ & & \\
\hline 8 & 1990 & Addenbrooke's Hospital, Cambridge, UK & 11 & & \\
\hline 9 & 1991-2004 & $\begin{array}{l}\text { New England Medical Center Hospitals, and Tutts } \\
\text { University School of Medicine, Boston, MA, USA }\end{array}$ & 12,13 & & \\
\hline 10 & 1993 & Eye Division of Olympia Medical Clinic, Tokyo, Japan & $14^{*}$ & & \\
\hline 11 & 1996 & $\begin{array}{l}\text { Massachusetts Eye and Ear Infirmary, Harvard Medical } \\
\text { School, Boston, MA, USA }\end{array}$ & 15 & & \\
\hline 12 & 1994 & Tokyo University School of Medicine, Tokyo, Japan & $16^{*}$ & & \\
\hline 13 & 1994 & Moorfields Eye Hospital, London, UK & & 17 & \\
\hline 14 & 1996 & $\begin{array}{l}\text { Centre Hospitalier Universitaire d'Angers, Angers, } \\
\text { France }\end{array}$ & 18 & & \\
\hline 15 & 1996-1997 & Wilmer Institute, Baltimore, MD, USA & 19,20 & & \\
\hline 16 & 1996-2000 & Gifu University Hospital, Gifu-shi, Japan & $\begin{array}{l}21^{*}, 22^{*} \\
23^{*}, 24^{*}\end{array}$ & $25^{*}$ & \\
\hline 17 & 1997-1999 & University of British Columbia, Vancouver, BC, Canada & $26^{*}, 27$ & & \\
\hline 18 & 1997-2000 & St Lucas Hospital, Amsterdam, the Netherlands & & 28,29 & \\
\hline 19 & 2001 & $\begin{array}{l}\text { Collaborative Normal-Tension Glaucoma Study, USA, } \\
\text { Canada, the Netherlands, Finland }\end{array}$ & $30 *$ & & \\
\hline 20 & 2000-2005 & Moorfields Eye Hospital, London, UK & $\begin{array}{l}31^{*}, 32^{*} \\
33^{*}\end{array}$ & & \\
\hline 21 & 2000-2006 & $\begin{array}{l}\text { Glaucoma Clinic, Nihon University Hospital, Tokyo, } \\
\text { Japan }\end{array}$ & $34^{*}, 35^{*}$ & & \\
\hline 22 & $2005-2008$ & University Eye Clinic, Basel, Switzerland & 36,37 & & \\
\hline 23 & $2002-2008$ & Advanced Glaucoma Intervention Study, USA & 38 & $\begin{array}{l}39,40, \\
41\end{array}$ & \\
\hline
\end{tabular}


Table 1: continued

\begin{tabular}{|c|c|c|c|c|c|}
\hline \multirow{2}{*}{$\begin{array}{l}\text { Cluster } \\
\text { number }\end{array}$} & \multirow{2}{*}{$\begin{array}{l}\text { Publication } \\
\text { year }\end{array}$} & \multirow[t]{2}{*}{ Source of study population } & \multicolumn{3}{|c|}{ Study references } \\
\hline & & & $\begin{array}{l}\text { Case } \\
\text { series }\end{array}$ & $\begin{array}{l}\text { Cohort } \\
\text { studies }\end{array}$ & $\begin{array}{l}\text { CC } \\
\text { studies }\end{array}$ \\
\hline 24 & 2000-2009 & $\begin{array}{l}\text { Queen Elisabeth Health Sciences Center, Halifax, NS, } \\
\text { Canada }\end{array}$ & 42,43 & $\begin{array}{l}44,45 \\
46\end{array}$ & \\
\hline 25 & $2000-2010$ & University of Washington, Seattle, WA, USA & $\begin{array}{l}47,48 \\
49\end{array}$ & & 50 \\
\hline 26 & 2001 & University of lowa, lowa City, IA, USA & 51 & & \\
\hline 27 & 2001 & Yamanashi Medical University, Yamanashi, Japan & & & $52^{*}$ \\
\hline 28 & 2001-2010 & Collaborative Initial Glaucoma Treatment Study, USA & 53 & 54,55 & \\
\hline 29 & 2002 & From population study in Saint Lucia, West Indies & 56 & & \\
\hline 30 & 2003-2007 & Wills Eye Hospital, Philadelphia, PA, USA & 57,58 & & \\
\hline 31 & 2003-2009 & Early Manifest Glaucoma Treatment Study, Sweden & 59 & $\begin{array}{l}60,61 \\
62,63\end{array}$ & \\
\hline 32 & 2005 & Gallego Institute of Ophthalmology, La Coruna, Spain & & 64 & \\
\hline 33 & 2005 & Bristol Eye Hospital, Bristol, UK & 65 & & \\
\hline 34 & 2005-2008 & Helsinki University Eye Hospital, Helsinki, Finland & 66,67 & & \\
\hline 35 & 2007 & $\begin{array}{l}\text { Sant' Andrea Hospital, La Sapienza II University, Roma, } \\
\text { Italy; University of Genova, Genova, Italy }\end{array}$ & 68 & & \\
\hline 36 & 2008 & National Taiwan University Hospital, Taipei, Taiwan & 69 & & \\
\hline 37 & 2008-2010 & Canadian Glaucoma Study, Canada & & 70,71 & \\
\hline 38 & 2008-2010 & Gallego Institute of Ophthalmology, La Coruna, Spain & & 72,73 & \\
\hline 39 & 2009 & Asan Medical Center, Seoul, Korea & $74^{*}$ & & \\
\hline 40 & 2009 & $\begin{array}{l}\text { Moorfields Eye Hospital and UCL Institute of Ophthal- } \\
\text { mology, London, UK }\end{array}$ & 75 & & \\
\hline 41 & $2009-2010$ & Hong Kong Eye Hospital, Hong Kong, China & $76^{*}, 77^{*}$ & & \\
\hline 42 & 2009-2010 & $\begin{array}{l}\text { New York Glaucoma Progression Study, New York, NY, } \\
\text { USA }\end{array}$ & $\begin{array}{l}78,79 \\
80,81 \\
82\end{array}$ & & \\
\hline 43 & 2010 & Samsung Medical Center, Seoul, Korea & $83^{*}$ & & \\
\hline 44 & 2010 & $\begin{array}{l}\text { Glaucoma Unit of the Aberdeen Royal Infirmary, } \\
\text { Aberdeen, UK }\end{array}$ & 84 & & \\
\hline 45 & 2010 & $\begin{array}{l}\text { Diagnostic Innovations in Glaucoma Study, Hamilton } \\
\text { Glaucoma Center, University of California, San Diego, } \\
\text { CA, USA }\end{array}$ & 85 & & \\
\hline
\end{tabular}




\section{Study references of Table 1}

1. Drance SM, Fairclough M, Butler DM, Kottler MS. The importance of disc hemorrhage in the prognosis of chronic open angle glaucoma. Arch Ophthalmol 1977;95:226-8.

2. Wilson R, Walker AM, Dueker DK, Crick RP. Risk factors for rate of progression of glaucomatous visual field loss: a computer-based analysis. Arch Ophthalmol 1982;100:737-41.

3. Crick RP, Newson RB, Shipley MJ, et al. The progress of the visual field in chronic simple glaucoma and ocular hypertension treated topically with pilocarpine or with timolol. Eye 1990;4:563-71.

4. Vossen J, Neubauer H. Long-term observations after trephination with scleral flap in glaucoma with threatened point of fixation. Ophthalmologica 1984;189:169-76.

5. Mikelberg FS, Schulzer M, Drance SM, Lau W. The rate of progression of scotomas in glaucoma. Am J Ophthalmol 1986;101:1-6.

6. Chauhan BC, Drance SM. The relationship between intraocular pressure and visual field progression in glaucoma. Graefes Arch Clin Exp Ophthalmol 1992;230:521-6.

7. Gramer E, Althaus G. Progression of glaucomatous visual field damage. Fortschr Ophthalmol 1988;85:620-5.

8. Gramer E, Althaus G. Einfluss des systolischen Blutdrucks auf die Lage der Gesichtsfeldausfalle in oberer und unterer Gesichtsfeldhalfte bei Patienten mit Glaucoma chronicum simplex. Ophthalmologe 1993;90:620-5.

9. Demailly P, Gruber D, Kretz G. Traitement du glaucome primitif chronique a angle ouvert. Resultats fonctionnels a long terme. J Fr Ophtalmol 1989;12:527-34.

10. Gliklich RE, Steinmann WC, Spaeth GL. Visual field change in low-tension glaucoma over a five-year follow-up. Ophthalmology 1989;96:316-20.

11. Watson PG, Jakeman C, Ozturk M, et al. The complications of trabeculectomy. (A 20-year follow-up). Eye 1990;4:425-38.

12. O'Brien C, Schwartz B, Takamoto T, Da Ching W. Intraocular pressure and the rate of visual field loss in chronic open-angle glaucoma. Am J Ophthalmol 1991;111:491-500.

13. Schwartz B, Takamoto T, Martin J. Increased Rate of Visual Field Loss Associated with Larger Initial Visual Field Threshold Values on Follow-Up of Open-Angle Glaucoma. J Glaucoma 2004;13:120-9.

14. Yoshikawa K, Inoue T, Inoue Y. Normal tension glaucoma: The value of predictive tests. Acta Ophthalmol (Copenh) 1993;71:463-70.

15. Siegner SW, Netland PA. Optic disc hemorrhages and progression of glaucoma. Ophthalmology 1996;103:1014-24.

16. Araie M, Sekine M, Suzuki Y, Koseki N. Factors contributing to the progression of visual field damage in eyes with normal-tension glaucoma. Ophthalmology 1994;101:1440-4.

17. Migdal C, Gregory W, Hitchings R. Long-term functional outcome after early surgery compared with laser and medicine in open-angle glaucoma. Ophthalmology 1994;101:1651-6; discussion 7.

18. Bresson Dumont $\mathrm{H}$, Bechetoille A. Role of arterial blood pressure and progression of glaucomatous damage. J Fr Ophtalmol 1996;19:435-42.

19. Smith SD, Katz J, Quigley HA. Analysis of progressive change in automated visual fields in glaucoma. Invest Ophthalmol Vis Sci 1996;37:1419-28.

20. Katz J, Gilbert D, Quigley HA, Sommer A. Estimating progression of visual field loss in glaucoma. Ophthalmology 1997;104:1017-25.

21. Daugeliene L, Yamamoto T, Kitazawa Y. Effect of trabeculectomy on visual field in progressive normaltension glaucoma. Jpn J Ophthalmol 1998;42:286-92.

22. Ishida K, Yamamoto $T$, Kitazawa $Y$. Clinical factors associated with progression of normal-tension glaucoma. J Glaucoma 1998;7:372-7. 
23. Daugeliene L, Yamamoto T, Kitazawa Y. Risk factors for visual field damage progression in normaltension glaucoma eyes. Graefes Arch Clin Exp Ophthalmol 1999;237:105-8.

24. Ishida K, Yamamoto T, Sugiyama K, Kitazawa Y. Disk hemorrhage is a significantly negative prognostic factor in normal-tension glaucoma. Am J Ophthalmol 2000;129:707-14.

25. Sawada A, Kitazawa Y, Yamamoto T, et al. Prevention of visual field defect progression with brovincamine in eyes with normal-tension glaucoma. Ophthalmology 1996;103:283-8.

26. Yamazaki Y, Drance SM. The relationship between progression of visual field defects and retrobulbar circulation in patients with glaucoma. Am J Ophthalmol 1997;124:287-95.

27. Graham SL, Drance SM. Nocturnal hypotension: role in glaucoma progression. Surv Ophthalmol 1999;43 Suppl 1:S10-6.

28. Rasher MT, Van Enden AD, Bakhcr D, Hoyng PFJ. Deterioration of visual fields in patients with glaucoma with and without optic disc hemorrhages. Arch Ophthalmol 1997;115:1257-62.

29. Rasker MT, van den Enden A, Bakker D, Hoyng PF. Rate of visual field loss in progressive glaucoma. Arch Ophthalmol 2000;118:481-8.

30. Drance S, Anderson DR, Schulzer M. Risk factors for progression of visual field abnormalities in normaltension glaucoma. Am J Ophthalmol 2001;131:699-708.

31. Membrey WL, Poinoosawmy DP, Bunce C, et al. Comparison of visual field progression in patients with normal pressure glaucoma between eyes with and without visual field loss that threatens fixation. $\mathrm{Br} J$ Ophthalmol 2000;84:1154-8.

32. Membrey WL, Bunce C, Poinoosawmy DP, et al. Glaucoma surgery with or without adjunctive antiproliferatives in normal tension glaucoma: 2 Visual field progression. Br J Ophthalmol 2001;85:696-701.

33. Aung $\mathrm{T}$, Rezaie $\mathrm{T}$, Okada K, et al. Clinical features and course of patients with glaucoma with the E50K mutation in the optineurin gene. Invest Ophthalmol Vis Sci 2005;46:2816-22.

34. Yamazaki Y, Hayamizu F, Tanaka C. Effects of long-term methylcobalamin treatment on the progression of visual field defects in normal-tension glaucoma. Curr Ther Res Clin Exp 2000;61:443-51.

35. Nakagami T, Yamazaki Y, Hayamizu F. Prognostic factors for progression of visual field damage in patients with normal-tension glaucoma. Jpn J Ophthalmol 2006;50:38-43.

36. Emre M, Orgul S, Haufschild T, et al. Increased plasma endothelin-1 levels in patients with progressive open angle glaucoma. Br J Ophthalmol 2005;89:60-3.

37. Kovalska M, Grieshaber MC, Schotzau A, et al. Detection of visual field progression in glaucoma. Klin Monatsbl Augenheilkd 2008;225:342-5.

38. Caprioli J, Coleman AL. Intraocular Pressure Fluctuation A Risk Factor for Visual Field Progression at Low Intraocular Pressures in the Advanced Glaucoma Intervention Study. Ophthalmology 2008;115:1123-9.

39. Blackwell B, Gaasterland D, Ederer F, et al. The Advanced Glaucoma Intervention Study (AGIS): 12. Baseline risk factors for sustained loss of visual field and visual acuity in patients with advanced glaucoma. Am J Ophthalmol 2002;134:499-512.

40. Nouri Mahdavi K, Hoffman D, Gaasterland D, Caprioli J. Prediction of visual field progression in glaucoma. Invest Ophthalmol Vis Sci 2004;45:4346-51.

41. Nouri Mahdavi K, Hoffman D, Coleman AL, et al. Predictive factors for glaucomatous visual field progression in the Advanced Glaucoma Intervention Study. Ophthalmology 2004;111:1627-35.

42. Soares AS, Artes PH, McCormick TA, et al. Retinal arterial diameter changes in progressive and nonprogressive glaucoma. J Glaucoma 2003;12:243-9.

43. Chauhan BC, Hutchison DM, LeBlanc RP, et al. Central corneal thickness and progression of the visual field and optic disc in glaucoma. Br J Ophthalmol 2005;89:1008-12.

44. Martinez Bello C, Chauhan BC, Nicolela MT, et al. Intraocular pressure and progression of glaucomatous visual field loss. Am J Ophthalmol 2000;129:302-8. 
45. Nicolela MT, McCormick TA, Drance SM, et al. Visual Field and Optic Disc Progression in Patients with Different Types of Optic Disc Damage: A Longitudinal Prospective Study. Ophthalmology 2003;110:217884.

46. Chauhan BC, Nicolela MT, Artes PH. Incidence and Rates of Visual Field Progression after Longitudinally Measured Optic Disc Change in Glaucoma. Ophthalmology 2009;116:2110-8.

47. Chen PP, Park RJ. Visual field progression in patients with initially unilateral visual field loss from chronic open-angle glaucoma. Ophthalmology 2000;107:1688-92.

48. Chen PP. Correlation of visual field progression between eyes in patients with open-angle glaucoma. Ophthalmology 2002;109:2093-9.

49. Chen PP, Cady RS, Mudumbai RC, Ngan R. Continued Visual Field Progression in Eyes With Prior Visual Field Progression in Patients With Open-Angle Glaucoma. J Glaucoma 2010;19:598-603.

50. Kim JW, Chen PP. Central corneal pachymetry and visual field progression in patients with open-angle glaucoma. Ophthalmology 2004;111:2126-32.

51. Kwon YH, Kim CS, Zimmerman MB, et al. Rate of visual field loss and long-term visual outcome in primary open-angle glaucoma. Am J Ophthalmol 2001;132:47-56.

52. Kashiwagi K, Hosaka O, Kashiwagi F, et al. Systemic circulatory parameters. comparison between patients with normal tension glaucoma and normal subjects using ambulatory monitoring. Jpn J Ophthalmol 2001;45:388-96.

53. Chen PP, Musch DC, Niziol LM, for the CSG. The Effect of Early Posttrabeculectomy Intraocular Pressure Spike in the Collaborative Initial Glaucoma Treatment Study. J Glaucoma 2011;20:211-4.

54. Lichter PR, Musch DC, Gillespie BW, et al. Interim clinical outcomes in the collaborative initial glaucoma treatment study comparing initial treatment randomized to medications or surgery. Ophthalmology 2001;108:1943-53.

55. Musch DC, Gillespie BW, Lichter PR, et al. Visual Field Progression in the Collaborative Initial Glaucoma Treatment Study. The Impact of Treatment and Other Baseline Factors. Ophthalmology 2009;116:2007.e1.

56. Wilson MR. Progression of visual field loss in untreated glaucoma patients and suspects in St Lucia, West Indies. Trans Am Ophthalmol Soc 2002;100:365-410.

57. Polansky JR, Juster RP, Spaeth GL. Association of the myocilin mt.1 promoter variant with the worsening of glaucomatous disease over time. Clin Genet 2003;64:18-27.

58. Siam GA, Monteiro De Barros DS, Gheith ME, et al. The amount of intraocular pressure rise during pharmacological pupillary dilatation is an indicator of the likelihood of future progression of glaucoma. Br J Ophthalmol 2007;91:1170-2.

59. Heijl A, Leske MC, Bengtsson B, et al. Measuring visual field progression in the Early Manifest Glaucoma Trial. Acta Ophthalmol Scand 2003;81:286-93.

60. Leske MC, Heijl A, Hussein M, et al. Factors for glaucoma progression and the effect of treatment: The early manifest glaucoma trial. Arch Ophthalmol 2003;121:48-56.

61. Leske MC, Heijl A, Hyman L, et al. Predictors of long-term progression in the early manifest glaucoma trial. Ophthalmology 2007;114:1965-72.

62. Bengtsson B, Leske MC, Yang Z, Heijl A. Disc hemorrhages and treatment in the early manifest glaucoma trial. Ophthalmology 2008;115:2044-8.

63. Heijl A, Bengtsson B, Hyman L, Leske MC. Natural History of Open-Angle Glaucoma. Ophthalmology 2009;116:2271-6.

64. Martinez A, Sanchez M. Predictive value of colour Doppler imaging in a prospective study of visual field progression in primary open-angle glaucoma. Acta Ophthalmol Scand 2005;83:716-22.

65. Spry PG, Sparrow JM, Diamond JP, Harris HS. Risk factors for progressive visual field loss in primary open angle glaucoma. Eye 2005;19:643-51. 
66. Ehrnrooth P, Puska P, Lehto I, Laatikainen L. Progression of visual field defects and visual loss in trabeculectomized eyes. Graefes Arch Clin Exp Ophthalmol 2005;243:741-7.

67. Harju M, Kurvinen L, Saari J, Vesti E. Blood flow in the peripapillary retina in exfoliation glaucoma. Clin Experiment Ophthalmol 2008;36:738-43.

68. Perdicchi A, lester M, Scuderi G, et al. Visual field damage and progression in glaucomatous myopic eyes. Eur J Ophthalmol 2007;17:534-7.

69. Lee YA, Shih YF, Lin LLK, et al. Association between high myopia and progression of visual field loss in primary open-angle glaucoma. J Formos Med Assoc 2008;107:952-7.

70. Chauhan BC, Mikelberg FS, Balaszi AG, et al. Canadian Glaucoma Study: 2. risk factors for the progression of open-angle glaucoma. Arch Ophthalmol 2008;126:1030-6.

71. Chauhan BC, Mikelberg FS, Artes PH, et al. Canadian Glaucoma Study: 3. Impact of Risk Factors and Intraocular Pressure Reduction on the Rates of Visual Field Change. Arch Ophthalmol 2010;128:1249-55.

72. Martinez A, Sanchez M. Effects of dorzolamide $2 \%$ added to timolol maleate $0.5 \%$ on intraocular pressure, retrobulbar blood flow, and the progression of visual field damage in patients with primary open-angle glaucoma: A single-center, 4-year, open-label study. Clin Ther 2008;30:1120-34.

73. Martinez A, Sanchez-Salorio M. Predictors for visual field progression and the effects of treatment with dorzolamide $2 \%$ or brinzolamide $1 \%$ each added to timolol $0.5 \%$ in primary open-angle glaucoma. Acta Ophthalmol Scand 2010;88:541-52.

74. Sung KR, Lee S, Park SB, et al. Twenty-four hour ocular perfusion pressure fluctuation and risk of normaltension glaucoma progression. Invest Ophthalmol Vis Sci 2009;50:5266-74.

75. Kotecha A, Spratt A, Bunce C, et al. Optic disc and visual field changes after trabeculectomy. Invest Ophthalmol Vis Sci 2009;50:4693-9.

76. Leung DYL, Tham CCY, Li FCH, et al. Silent Cerebral Infarct and Visual Field Progression in Newly Diagnosed Normal-Tension Glaucoma. A Cohort Study. Ophthalmology 2009;116:1250-6.

77. Leung DYL, Li FCH, Kwong YYY, et al. Simvastatin and Disease Stabilization in Normal Tension Glaucoma: A Cohort Study. Ophthalmology 2010;117:471-6.

78. De Moraes CG, Prata TS, Liebmann CA, et al. Spatially consistent, localized visual field loss before and after disc hemorrhage. Invest Ophthalmol Vis Sci 2009;50:4727-33.

79. De Moraes CG, Prata TS, Tello C, et al. Glaucoma with early visual field loss affecting both hemifields and the risk of disease progression. Arch Ophthalmol 2009;127:1129-34.

80. Ahrlich KG, De Moraes CG, Teng CC, et al. Visual field progression differences between normal-tension and exfoliative high-tension glaucoma. Invest Ophthalmol Vis Sci 2010;51:1458-63.

81. Prata TS, De Moraes CGV, Teng CC, et al. Factors Affecting Rates of Visual Field Progression in Glaucoma Patients with Optic Disc Hemorrhage. Ophthalmology 2010;117:24-9.

82. Teng CC, De Moraes CGV, Prata TS, et al. beta-Zone Parapapillary Atrophy and the Velocity of Glaucoma Progression. Ophthalmology 2010;117:909-15.

83. Sohn SW, Song JS, Kee C. Influence of the extent of myopia on the progression of normal-tension glaucoma. Am J Ophthalmol 2010;149:831-8.

84. Ang GS, Mustafa MS, Scott N, et al. Perimetric Progression in Open Angle Glaucoma and the Visual Field Index (VFI). J Glaucoma 2011;20:223-7.

85. Medeiros FA, Alencar LM, Sample PA, et al. The Relationship between Intraocular Pressure Reduction and Rates of Progressive Visual Field Loss in Eyes with Optic Disc Hemorrhage. Ophthalmology 2010;117:2061-6. 
Table 2: Quality scores of the 85 included studies

\begin{tabular}{|c|c|c|c|c|}
\hline Quality items from NICE checklist ${ }^{13}$ & $\begin{array}{l}\text { No. } \\
\text { Yes }\end{array}$ & $\begin{array}{l}\text { No. } \\
\text { No }\end{array}$ & $\begin{array}{l}\text { No. } \\
\text { Unclear }\end{array}$ & Explanation for scoring studies \\
\hline $\begin{array}{l}\text { 1. The study sample represents the } \\
\text { population of interest with regard } \\
\text { to key characteristics, sufficient to } \\
\text { limit bias to the results. }\end{array}$ & $\begin{array}{c}73 \\
(86 \%)\end{array}$ & $\begin{array}{c}0 \\
(0 \%)\end{array}$ & $\begin{array}{c}12 \\
(14 \%)\end{array}$ & $\begin{array}{l}\text { We rated studies that investigated only } \\
\text { glaucoma patients with established } \\
\text { baseline visual field loss. Baseline } \\
\text { characteristics (i.e., age, gender, and } \\
\text { visual field loss) should be adequately } \\
\text { described. }\end{array}$ \\
\hline $\begin{array}{l}\text { 2. Loss to follow-up is unrelated to } \\
\text { key characteristics (i.e., the study } \\
\text { data adequately represent the } \\
\text { sample), sufficient to limit } \\
\text { potential bias. }\end{array}$ & $\begin{array}{c}3 \\
(4 \%)\end{array}$ & $\begin{array}{c}70 \\
(82 \%)\end{array}$ & $\begin{array}{c}12 \\
(14 \%)\end{array}$ & $\begin{array}{l}\text { We rated cohort studies that either } \\
\text { reported similar baseline characteristics in } \\
\text { the group lost to follow-up and the } \\
\text { analyzed group, or had no loss to follow- } \\
\text { up. By definition, case series and case- } \\
\text { control studies were rated as "No". }\end{array}$ \\
\hline $\begin{array}{l}\text { 3. The prognostic factor of interest } \\
\text { is adequately measured in study } \\
\text { participants, sufficient to limit bias. }\end{array}$ & $\begin{array}{c}85 \\
(100 \%)\end{array}$ & $\begin{array}{c}0 \\
(0 \%)\end{array}$ & $\begin{array}{c}0 \\
(0 \%)\end{array}$ & $\begin{array}{l}\text { We rated studies that measured prognos- } \\
\text { tic factors in a treated or untreated } \\
\text { glaucoma patient before the follow-up } \\
\text { period in which visual field progression } \\
\text { could occur. }\end{array}$ \\
\hline $\begin{array}{l}\text { 4. The outcome of interest is } \\
\text { adequately measured in study } \\
\text { participants, sufficient to limit bias. }\end{array}$ & $\begin{array}{c}73 \\
(86 \%)\end{array}$ & $\begin{array}{c}11 \\
(13 \%)\end{array}$ & $\begin{array}{c}1 \\
(1 \%)\end{array}$ & $\begin{array}{l}\text { We rated the outcome as adequately } \\
\text { measured if the same type of perimeter } \\
\text { was used in all participants and if the } \\
\text { perimeter used was an automated } \\
\text { perimeter. }\end{array}$ \\
\hline $\begin{array}{l}\text { 5. Important potential confound- } \\
\text { ers are appropriately accounted } \\
\text { for, limiting potential bias with } \\
\text { respect to the prognostic factor of } \\
\text { interest. }\end{array}$ & $\begin{array}{c}53 \\
(62 \%)\end{array}$ & $\begin{array}{c}32 \\
(38 \%)\end{array}$ & $\begin{array}{c}0 \\
(0 \%)\end{array}$ & $\begin{array}{l}\text { We rated studies that performed a } \\
\text { multivariable analysis. }\end{array}$ \\
\hline $\begin{array}{l}\text { 6. The statistical analysis is } \\
\text { appropriate for the design of the } \\
\text { study, limiting potential for the } \\
\text { presentation of invalid results. }\end{array}$ & $\begin{array}{c}74 \\
(87 \%)\end{array}$ & $\begin{array}{c}11 \\
(13 \%)\end{array}$ & $\begin{array}{c}0 \\
(0 \%)\end{array}$ & $\begin{array}{l}\text { We rated studies that adequately } \\
\text { described the statistical analysis in a way } \\
\text { that it is reproducible for others. All } \\
\text { candidate variables considered for the } \\
\text { analysis should be listed. }\end{array}$ \\
\hline
\end{tabular}

NICE = National Institute for Health and Clinical Excellence 
Table 3: Sample evidence table summarizing the study results of a prognostic factor

\begin{tabular}{|c|c|c|c|c|c|c|c|}
\hline \multirow{2}{*}{$\begin{array}{l}\text { Prognostic } \\
\text { factor " } X \text { " }\end{array}$} & \multicolumn{3}{|c|}{ More progression } & \multirow[b]{2}{*}{ No relation } & \multicolumn{3}{|c|}{ Less progression } \\
\hline & Significant & & $\begin{array}{c}\text { Non- } \\
\text { significant }\end{array}$ & & $\begin{array}{c}\text { Non- } \\
\text { significant }\end{array}$ & & Significant \\
\hline $\begin{array}{l}\text { Univariate analysis } \\
(n=10)\end{array}$ & $\underline{1 a, 1 b^{+}}$ & $2^{\ddagger}$ & $3,4,5^{*}, 6$ & 7,8 & $9 *$ & & \\
\hline No. of clusters & 1 & 1 & 4 & 2 & 1 & 0 & 0 \\
\hline No. of studies & 2 & 1 & 4 & 2 & 1 & 0 & 0 \\
\hline No. of patients & 130 & 140 & 1032 & 325 & 48 & 0 & 0 \\
\hline $\begin{array}{l}\text { Multivariable analysis } \\
(n=8)\end{array}$ & $\underline{1 a, 1 b^{\dagger}}, 4$ & & $3,5^{*}$ & $2,6,9 *$ & & & \\
\hline No. of clusters & 2 & 0 & 2 & 3 & 0 & 0 & 0 \\
\hline No. of studies & 3 & 0 & 2 & 3 & 0 & 0 & 0 \\
\hline No. of patients & 757 & 0 & 350 & 243 & 0 & 0 & 0 \\
\hline
\end{tabular}

The evidence table summarizes the univariate and multivariable study results of prognostic factor "X". These tables were used to determine the extent to which prognostic factors were associated with glaucomatous visual field progression. Each number in the table represents a study. Studies are classified by the direction and the statistical significance $(P \leq 0.05)$ of the association between the prognostic factor and visual field progression. The number of study clusters, studies, and patients is summarized below. Prognostic factor " $X "$ was judged to be probably associated with more visual field progression in studies with all types of open-angle glaucoma and not associated with progression in normal-tension glaucoma studies.

* Studies 5 and 9 comprised a majority of normal-tension glaucoma patients.

'Studies $1 \mathrm{a}$ and $1 \mathrm{~b}$ are underlined because they belong to the same study cluster. Their results are likely to be correlated.

${ }^{\ddagger}$ Study 2 was classified as falling between a significant and non-significant association because it used two methods to assess progression: one resulted in a statistically significant association and the other resulted in a non-significant association. 


\section{Summary of evidence}

\section{Judgment procedure}

The 85 included studies were published between 1977 and 2010 and consisted mainly of case series (73\%) (see Table 1). From these studies, we classified the results of 103 different prognostic factors in evidence tables (e.g., Table 3). Fifty-three studies (62\%) performed a multivariable analysis. From a total of 795 conclusions in the articles, 471 (59\%) were univariate results and 324 (41\%) were multivariable results.

We consistently applied the following guidelines in classifying the prognostic factors. We assigned "no association" to factors with a consistent pattern of "No relation." Factors with very inconsistent results from different studies were also assigned "no association." "Possible association" was assigned to factors with a weak tendency towards more or Less progression with a single significant result. "Probable association" was assigned to factors with an obvious tendency towards more or Less progression in a moderate number of studies with several studies having significant results. However, if only a few significant results appeared in a very consistent pattern, this factor was also assigned a "probable association." "Definite association" was assigned to factors with a very consistent pattern of numerous studies with multiple statistically significant results.

Our judgments were generally consistent $(k=0.89)$. In all cases of differences, we maintained the second judgment, which suggests a learning effect during the first consensus round.

The judgment procedure of the association of prognostic factors with visual field progression resulted in one definite association (1\%), seven probable associations (6.8\%), 24 possible associations (23.3\%), and 71 times no association (68.9\%) (see Tables 4 and 5).

Eighteen factors were judged to have a different association with progression in NTG studies compared with studies investigating all types of OAG. These results are presented separately and implied one definite association, two probable associations, six possible associations, and nine times no association (see Tables 4 and 5). We lumped the results for all other factors and reported them as factors studied in OAG.

\section{Definite and probable prognostic factors}

We identified one definite and seven probable prognostic factors for glaucomatous visual field progression in OAG. Additionally, in NTG specific studies we identified one definite and two probable prognostic factors (see Table 4). The evidence tables of these 
factors are shown in Table 6 (see appendix Chapter 2). Higher age was definitely associated with More progression in OAG patients and the presence of disc hemorrhages was definitely associated with More progression in NTG patients. Furthermore, more baseline visual field loss, higher baseline IOP and presence of exfoliation syndrome were probably associated with more visual field progression in OAG. Finally, six other factors were probably associated with visual field progression, but the judgment was based on a low number of studies. Central corneal thickness (CCT), ocular blood flow resistivity index, blood anticardiolipin antibody, peripapillary atrophy (for NTG), proven previous visual field progression, and stroke (for NTG) were probably associated with progression. Thirty factors were possibly associated with visual field progression and are shown in Table 4. The evidence tables of these factors are shown in Table 6 (see appendix Chapter 2).

\section{Unlikely prognostic factors}

Most prognostic factors were judged to have no association with glaucomatous visual field progression, but this was based on a limited number of studies (Table 5). The evidence tables of these factors are shown in Table 6 (see appendix Chapter 2), except for factors that were studied only once or twice. We identified eight prognostic factors that were addressed by ten or more studies, but still showed no evidence of an association with visual field progression: family history of glaucoma, atherosclerosis, systemic hypertension, visual acuity, gender (for NTG), systolic blood pressure, myopic refractive error (for NTG), and Raynaud's phenomenon. These factors were rated as unlikely prognostic factors.

\section{Heterogeneity}

We noticed a considerable amount of heterogeneity in three probable prognostic factors for OAG: baseline visual field loss, baseline IOP and CCT. Although more baseline visual field loss was associated with More progression in most studies, six out of 36 studies showed that a better baseline visual field was associated with More progression in the multivariable analysis. ${ }^{19-24}$ However, only one study, part of the Advanced Glaucoma Intervention Study (AGIS), found a statistically significant result. ${ }^{24}$ We observed no difference between the mean degree of baseline field loss per study and the direction of association. For baseline IOP, two out of 17 studies found that a lower baseline IOP was associated with more visual field progression during follow-up in the multivariable analysis. ${ }^{22,}{ }^{25}$ However, these results were not statistically significant. Correlated, both studies investigated baseline IOP as a prognostic factor in the AGIS population. A higher mean IOP and IOP fluctuation during follow-up resulted in More progression in these studies. ${ }^{22,25}$ The heterogeneity we observed for CCT was mainly ascribed to two out of five studies pointing at the opposite direction of the association in the univariate analy- 
sis. ${ }^{26,27}$ Because both studies applied a univariate pre-selection of predictors and univariate results were not statistically significant, CCT was not tested in the multivariable model. The other three studies showed that a thinner CCT was predictive of more visual field progression in univariate and multivariable analyses. ${ }^{28-30}$ Important to note here is that these three studies were clustered in the New York Glaucoma Progression Study (i.e., cluster 42 in Table 1).

We noticed heterogeneity for the following two unlikely prognostic factors: visual acuity and systolic blood pressure. For visual acuity, only one out of 14 studies found a significant association between visual acuity and visual field progression. ${ }^{24}$ Significantly less progression was found in patients with a better baseline visual acuity. However, the results of this study differed between the two treatment groups, with the other group showing a non-significant result. ${ }^{24}$ All other studies found only non-significant or no associations for this factor. In the multivariable results for systolic blood pressure, we found two out of ten studies showing significantly more progression with a higher systolic blood pressure. ${ }^{31,32}$ These studies were derived from the same study cluster from Gifu University Hospital (i.e., cluster 16 in Table 1). One other study found significantly less progression with higher blood pressures and was derived from the same study cluster. ${ }^{33}$

In 14 other presumed prognostic factors, we noticed considerable heterogeneity: gender (for OAG), diabetes, untreated IOP, diastolic blood pressure, IOP fluctuation, migraine, NTG, mean diurnal IOP, duration of disease, diurnal IOP fluctuation, mean arterial blood pressure, ocular blood flow peak systolic velocity, degree of optic nerve cupping, and thyroid disease. 
Table 4: Prognostic factors associated with glaucomatous visual field progression

\begin{tabular}{|c|c|c|c|c|c|c|}
\hline \multirow[t]{2}{*}{ Prognostic factors } & \multirow{2}{*}{$\begin{array}{l}\text { Diagnostic } \\
\text { group }\end{array}$} & \multirow{2}{*}{$\begin{array}{c}\text { Association } \\
\text { 1=no, 2=possible, } \\
\text { 3=probable, } \\
\text { 4=definite }\end{array}$} & \multirow{2}{*}{$\begin{array}{l}\text { Direction } \\
\text { Increased } \\
\text { progression } \\
\text { with: }\end{array}$} & \multicolumn{3}{|c|}{ Number of studies } \\
\hline & & & & $\begin{array}{l}\text { Univariate } \\
\text { analysis }\end{array}$ & $\begin{array}{c}\text { Multivariable } \\
\text { analysis }\end{array}$ & Total \\
\hline Age & OAG & 4 & Older age & 39 & 26 & 47 \\
\hline Disc hemorrhages & NTG & 4 & Presence & 5 & 6 & 7 \\
\hline Baseline visual field loss & OAG & 3 & More loss & 47 & 36 & 61 \\
\hline Baseline IOP & OAG & 3 & Higher IOP & 22 & 17 & 28 \\
\hline Exfoliation syndrome & OAG & 3 & Presence & 11 & 6 & 13 \\
\hline $\begin{array}{l}\text { Central corneal } \\
\text { thickness }\end{array}$ & OAG & 3 & Thinner & 5 & 3 & 5 \\
\hline OBF resistivity index & OAG & 3 & Higher index & 3 & 3 & 3 \\
\hline $\begin{array}{l}\text { Anticardiolipin antibody } \\
\text { in blood }\end{array}$ & OAG & 3 & Higher level & 2 & 1 & 2 \\
\hline Peripapillary atrophy & NTG & 3 & Presence & 0 & 2 & 2 \\
\hline $\begin{array}{l}\text { Previous visual field } \\
\text { progression }\end{array}$ & OAG & 3 & $\begin{array}{l}\text { More progres- } \\
\text { sion }\end{array}$ & 2 & 1 & 2 \\
\hline Stroke & NTG & 3 & Presence & 2 & 2 & 2 \\
\hline Gender & OAG & 2 & Female sex & 23 & 17 & 31 \\
\hline Cup disc ratio & OAG & 2 & Higher ratio & 14 & 7 & 17 \\
\hline Age & NTG & 2 & Older age & 10 & 11 & 16 \\
\hline $\begin{array}{l}\text { Myopic refractive error } \\
\text { (spherical equivalent) }\end{array}$ & OAG & 2 & Higher error & 12 & 10 & 16 \\
\hline Diabetes & OAG & 2 & Presence & 9 & 5 & 12 \\
\hline African descent & OAG & 2 & Presence & 7 & 5 & 9 \\
\hline Baseline untreated IOP & OAG & 2 & Higher IOP & 6 & 6 & 9 \\
\hline Disc hemorrhages & OAG & 2 & Presence & 7 & 3 & 8 \\
\hline Diastolic blood pressure & OAG & 2 & Lower pressure & 5 & 4 & 6 \\
\hline Systemic hypertension & NTG & 2 & Presence & 4 & 4 & 6 \\
\hline $\begin{array}{l}\text { Recovery rate from cold } \\
\text { exposure test }\end{array}$ & NTG & 2 & Lower rate & 0 & 5 & 5 \\
\hline Baseline IOP fluctuation & OAG & 2 & $\begin{array}{l}\text { Higher fluctua- } \\
\text { tion }\end{array}$ & 3 & 2 & 4 \\
\hline Migraine & NTG & 2 & Presence & 4 & 1 & 4 \\
\hline $\begin{array}{l}\text { Ocular perfusion } \\
\text { pressure (overall) }\end{array}$ & OAG & 2 & Lower pressure & 3 & 3 & 4 \\
\hline Pulse rate & OAG & 2 & Higher rate & 1 & 2 & 3 \\
\hline $\begin{array}{l}\text { Central corneal } \\
\text { thickness }\end{array}$ & NTG & 2 & Thinner & 3 & 2 & 3 \\
\hline
\end{tabular}


Table 4: continued

\begin{tabular}{|c|c|c|c|c|c|c|}
\hline \multirow[t]{2}{*}{ Prognostic factors } & \multirow{2}{*}{$\begin{array}{l}\text { Diagnostic } \\
\text { group }\end{array}$} & \multirow{2}{*}{$\begin{array}{l}\text { Association } \\
\text { 1=no, 2=possible, } \\
\text { 3=probable, } \\
\text { 4=definite }\end{array}$} & \multirow{2}{*}{$\begin{array}{l}\text { Direction } \\
\text { Increased } \\
\text { progression } \\
\text { with: }\end{array}$} & \multicolumn{3}{|c|}{ Number of studies } \\
\hline & & & & $\begin{array}{l}\text { Univariate } \\
\text { analysis }\end{array}$ & $\begin{array}{c}\text { Multivariable } \\
\text { analysis }\end{array}$ & Total \\
\hline $\begin{array}{l}\text { Infracentral scotoma } \\
\text { localization }\end{array}$ & OAG & 2 & Presence & 3 & 0 & 3 \\
\hline Peripapillary atrophy & OAG & 2 & Presence & 2 & 2 & 3 \\
\hline $\begin{array}{l}\text { Normal tension } \\
\text { glaucoma }\end{array}$ & OAG & 2 & Absence & 3 & 0 & 3 \\
\hline $\begin{array}{l}\text { OBF end diastolic } \\
\text { velocity }\end{array}$ & OAG & 2 & Lower velocity & 2 & 2 & 2 \\
\hline $\begin{array}{l}\text { Systolic ocular perfusion } \\
\text { pressure }\end{array}$ & OAG & 2 & Lower pressure & 1 & 2 & 2 \\
\hline $\begin{array}{l}\text { Family history of } \\
\text { diabetes }\end{array}$ & OAG & 2 & Absence & 1 & 0 & 1 \\
\hline $\begin{array}{l}\text { Variant of myocilin } \\
(\mathrm{mt} .1(+))\end{array}$ & OAG & 2 & Presence & 0 & 1 & 1 \\
\hline Asian descent & OAG & 2 & Absence & 1 & 0 & 1 \\
\hline $\begin{array}{l}\text { Red blood cell distribu- } \\
\text { tion width }\end{array}$ & OAG & 2 & Lower width & 1 & 0 & 1 \\
\hline Threat to fixation & NTG & 2 & Presence & 1 & 1 & 1 \\
\hline $\begin{array}{l}\text { Outflow facility/ } \triangle \mathrm{IOP} \\
\text { water drinking test/ } \\
\text { diurnal } \triangle \mathrm{IOP}\end{array}$ & OAG & 2 & $\downarrow / \uparrow / \uparrow$ & 1 & 0 & 1 \\
\hline $\begin{array}{l}\text { IOP increase after } \\
\text { pupillary dilatation }\end{array}$ & OAG & 2 & Higher increase & 1 & 0 & 1 \\
\hline $\begin{array}{l}\text { Highest IOP ever } \\
\text { recorded before } \\
\text { baseline }\end{array}$ & OAG & 2 & Higher IOP & 1 & 0 & 1 \\
\hline $\begin{array}{l}\text { Ocular perfusion } \\
\text { pressure fluctuation }\end{array}$ & OAG & 2 & $\begin{array}{l}\text { Higher fluctua- } \\
\text { tion }\end{array}$ & 1 & 1 & 1 \\
\hline
\end{tabular}

All prognostic factors investigated in the literature are ranked by the strength of their association with visual field progression (third column) and the number of studies investigating each factor (seventh column). The fourth column indicates the direction of association between the prognostic factor and visual field progression.

OAG = open-angle glaucoma, NTG = normal-tension glaucoma, IOP = intraocular pressure, OBF = ocular blood flow . 
Table 5: Prognostic factors without evidence of an association with glaucomatous visual field progression

\begin{tabular}{|c|c|c|c|c|c|c|}
\hline \multirow{2}{*}{$\begin{array}{l}\text { Prognostic } \\
\text { factors }\end{array}$} & \multirow{2}{*}{ Diagnostic group } & \multirow{2}{*}{$\begin{array}{l}\text { Association } \\
\text { 1=no, 2=possible, } \\
\text { 3=probable, } \\
\text { 4=definite }\end{array}$} & \multirow{2}{*}{$\begin{array}{l}\text { Direction } \\
\text { Increased } \\
\text { progression } \\
\text { with: }\end{array}$} & \multicolumn{3}{|c|}{ Number of studies } \\
\hline & & & & $\begin{array}{c}\text { Univariate } \\
\text { analysis }\end{array}$ & $\begin{array}{c}\text { Multivariable } \\
\text { analysis }\end{array}$ & Total \\
\hline Family history of glaucoma & OAG & 1 & - & 12 & 7 & 16 \\
\hline Atherosclerosis & OAG & 1 & - & 12 & 4 & 14 \\
\hline Systemic hypertension & OAG & 1 & - & 11 & 6 & 14 \\
\hline Visual acuity & OAG & 1 & - & 8 & 7 & 14 \\
\hline Gender & NTG & 1 & - & 9 & 6 & 13 \\
\hline Systolic blood pressure & OAG & 1 & - & 8 & 10 & 12 \\
\hline $\begin{array}{l}\text { Myopic refractive error } \\
\text { (spherical equivalent) }\end{array}$ & NTG & 1 & - & 6 & 8 & 11 \\
\hline Raynaud's phenomenon & OAG & 1 & - & 9 & 2 & 10 \\
\hline Baseline IOP & NTG & 1 & - & 4 & 7 & 8 \\
\hline Diabetes & NTG & 1 & - & 4 & 4 & 8 \\
\hline Migraine & OAG & 1 & - & 7 & 2 & 8 \\
\hline Smoking & OAG & 1 & - & 4 & 3 & 6 \\
\hline Diastolic blood pressure & NTG & 1 & - & 2 & 4 & 6 \\
\hline Baseline untreated IOP & NTG & 1 & - & 3 & 6 & 6 \\
\hline Mean diurnal IOP & OAG & 1 & - & 2 & 6 & 6 \\
\hline $\begin{array}{l}\text { Low blood pressure } \\
\text { tendency }\end{array}$ & OAG & 1 & - & 6 & 1 & 6 \\
\hline Duration of disease & OAG & 1 & - & 5 & 3 & 5 \\
\hline Diurnal IOP fluctuation & OAG & 1 & - & 2 & 3 & 5 \\
\hline $\begin{array}{l}\text { Mean ocular perfusion } \\
\text { pressure }\end{array}$ & OAG & 1 & - & 4 & 3 & 5 \\
\hline Right-hand eye & OAG & 1 & - & 5 & 0 & 5 \\
\hline Educational level & OAG & 1 & - & 1 & 4 & 4 \\
\hline $\begin{array}{l}\text { Minimum IOP of diurnal } \\
\text { variation }\end{array}$ & OAG & 1 & - & 2 & 2 & 4 \\
\hline $\begin{array}{l}\text { Maximum IOP of diurnal } \\
\text { variation }\end{array}$ & OAG & 1 & - & 2 & 2 & 4 \\
\hline Major hypotension & OAG & 1 & - & 3 & 1 & 4 \\
\hline Age at diagnosis & OAG & 1 & - & 3 & 2 & 3 \\
\hline MAP & OAG & 1 & - & 2 & 2 & 3 \\
\hline Disc size & OAG & 1 & - & 3 & 1 & 3 \\
\hline Threat to fixation & OAG & 1 & - & 3 & 0 & 3 \\
\hline Cardiac arrhythmia & OAG & 1 & - & 3 & 1 & 3 \\
\hline High cholesterol levels & OAG & 1 & - & 3 & 1 & 3 \\
\hline
\end{tabular}


Table 5: continued

\begin{tabular}{|c|c|c|c|c|c|}
\hline \multirow{2}{*}{$\begin{array}{l}\text { Prognostic } \\
\text { factors }\end{array}$} & \multirow{2}{*}{$\begin{array}{c}\text { Association } \\
\text { 1=no, } 2=\text { possible, } \\
\text { 3=probable, } \\
\text { 4=definite }\end{array}$} & \multirow{2}{*}{$\begin{array}{l}\text { Direction } \\
\text { Increased } \\
\text { progression } \\
\text { with: }\end{array}$} & \multicolumn{3}{|c|}{ Number of studies } \\
\hline & & & $\begin{array}{l}\text { Univariate } \\
\text { analysis }\end{array}$ & $\begin{array}{l}\text { Multivariable } \\
\text { analysis }\end{array}$ & Total \\
\hline $\begin{array}{l}\text { Focal ischemic disc } \\
\text { pathology }\end{array}$ & 1 & - & 1 & 2 & 3 \\
\hline $\begin{array}{l}\text { Family history of stroke or vascular hyper- } \\
\text { tension, Marital status, OBF peak systolic } \\
\text { velocity, Previous cataract surgery, Degree } \\
\text { of optic nerve cupping, Fellow eye disease, } \\
\text { Baseline target IOP, Obstructive sleep apnea, } \\
\text { Thyroid disease, Diastolic ocular perfusion } \\
\text { pressure, Pupil size, Visual field reliability } \\
\text { indices, RNFL thickness, Pigment glaucoma }\end{array}$ & ' & - & - & - & 2 \\
\hline $\begin{array}{l}\text { Alcohol intake, Family history of diabetes } \\
\text { (NTG), OPTN E5OK mutation, African descent } \\
\text { (NTG), Non-European Descent, Multiple } \\
\text { blood values, Diurnal MAP fluctuation, } \\
\text { Nocturnal MAP fluctuation, 24-hour MAP } \\
\text { fluctuation, Recovery rate from cold } \\
\text { exposure test, Finger blood flow, 24-hour } \\
\text { peak MAP, 24-hour trough MAP, Lens } \\
\text { opacities, Iris color, Previous optic disc } \\
\text { change, Postoperative IOP spike } \geq 5 \text { mmHg, } \\
\text { Anemia, Asthma, Blood transfusion, Fatigue } \\
\text { or weakness, Major surgery, Malignancy, } \\
\text { Muscle tremor or weakness, Nonmigraine } \\
\text { headache, Psychological symptoms, Renal } \\
\text { stones, Stroke, Sensorineural hearing loss, } \\
\text { Any systemic disease, Vomiting or diarrhea, } \\
\text { Ocular perfusion pressure (NTG), Pupil } \\
\text { response, Refractive error in cylinders, } \\
\text { Retinal artery narrowing }\end{array}$ & 1 & - & - & - & 1 \\
\hline
\end{tabular}

All prognostic factors investigated in the literature are ranked by the strength of their association with visual field progression (third column) and the number of studies investigating each factor (seventh column). The fourth column indicates the direction of association between the prognostic factor and visual field progression.

$\mathrm{OAG}=$ open-angle glaucoma, NTG = normal-tension glaucoma, $\mathrm{IOP}=$ intraocular pressure, $\mathrm{OBF}=$ ocular blood flow, MAP = mean arterial blood pressure. 


\section{Discussion}

This review article provides a systematic overview of evidence of all studied prognostic factors for glaucomatous visual field progression. Using semi-quantitative analysis, we classified two factors as definitely associated, nine as probably, and 30 as possibly associated with glaucomatous visual field progression (Table 4).

To create our review, we searched for a method that would go beyond the existing nonsystematic, narrative reviews. We did not do a formal meta-analysis because of the large variety in methods and approaches used by the studies. Instead, we summarized the knowledge on prognostic factors systematically and objectively, using a semiquantitative approach. We conducted a systematic literature search, consistently applied established selection criteria to include relevant articles, and summarized all study results in evidence tables. Following established assessment guidelines, four investigators individually assessed the evidence on each factor, had a consensus round, and repeated the entire process a month later. Because our investigators did not know which factor was under investigation or the study source, all prognostic factors were assessed under similar blind conditions to prevent subjective bias from tacit knowledge or convictions. Furthermore, for each factor, the investigators looked at multivariable results, which were corrected for the effects of other prognostic factors and were weighted more heavily than univariate results. Our approach also enabled us to include more studies than a formal meta-analysis could. Many studies included in our semiquantitative analysis did not report the effect size of a prognostic factor if it did not test statistically significant. Formal meta-analysis would have excluded these studies, because this analysis form requires an effect size. Our semi-quantitative approach did not aim to classify factors as true or not true but to rank them according to their likelihood of being prognostic. The results show the validity of our method, since the factors we found most likely to be associated with progression (age, disc hemorrhages, baseline visual field loss, intraocular pressure and exfoliation syndrome) will be recognized as such by many glaucoma specialists.

A previous review by Julvez and colleagues has shown that the methodological quality of studies on prognostic factors is generally moderate. ${ }^{11}$ In the current review, we used study inclusion criteria that contained several quality aspects. All of the included studies analyzed at least 25 glaucoma patients during a minimal follow-up period of three years and used quantitative methods to assess progression. We further rated study quality with the NICE-recommended checklist developed for quality assessment of prognosis studies. ${ }^{13}$ Most included studies had good scores for five of the six items (Table 2). However, for item 2 (loss to follow-up) we found only three cohort studies that indicat- 
ed No relation between key characteristics and loss to follow-up. ${ }^{33-35}$ Most included studies were case series or case-control studies, which we considered valid to identify prognostic factors since they compared patients with progression to patients without progression. We also compared our article search and selection process with the review by Julvez and colleagues, which included 47 articles. $^{11}$ Twenty-eight studies that they included were not included in our review. After studying those articles, we decided to still exclude them mainly because 13 did not study baseline prognostic factors for visual field progression, four did not distinguish eyes with and without baseline visual field loss in the analyses, and four used an unknown or qualitative method to assess visual field progression. On the other hand, we included 66 more studies than Julvez and colleagues did, including 28 studies published in the same period covered by Julvez and colleagues in their search. ${ }^{11}$

Studies that investigated NTG patients were initially judged separately, because we presumed to find different prognostic factors for NTG. However, current literature argues that both OAG with higher and lower IOPs represent one disease continuum. ${ }^{36}$ The distinction between normal and high IOP is largely arbitrary and has a historical rather than a scientific origin. ${ }^{37}$ In the 20 studies with a majority of NTG patients, we were dependent on the study-specific definitions of NTG. To identify different prognostic factors for OAG with higher and lower IOPs, it would have been ideal if subgroup analyses had been available. These analyses have been scarcely performed in the literature. Of the 18 factors judged to have a different association with progression for NTG, the largest discrepancy (i.e., a difference of two classes) was found for age, optic disc hemorrhages, baseline IOP, and stroke. Age was definitely associated with progression in 47 OAG studies and was possibly associated in 16 NTG studies. This could indicate an increasing vulnerability to glaucomatous damage during life, particularly in OAG with higher IOPs. However, this does not mean that older patients should always be treated more aggressively. At the same time older patients have a shorter life expectancy that decreases their risk of end-of-life visual disability. Optic disc hemorrhages were found to be more associated with progression in NTG. This may indicate different mechanisms of optic nerve damage between OAG with higher and lower IOPs. Disc hemorrhages may be a sign of the local vascular abnormalities that tend to be associated with NTG. ${ }^{36}$ For baseline IOP, we found a stronger association in OAG studies. This is likely caused by the higher IOP variation in the OAG population compared with a subgroup of NTG patients. Stroke was probably associated with progression in NTG based on two correlated studies. ${ }^{38,39}$ For OAG, only one study investigated this factor and found No relation between stroke and progression. ${ }^{34}$ These results suggest that stroke and NTG may share common pathogenetic pathways. However, more research is necessary to fully elucidate the importance of stroke for glaucomatous progression. 
It is important to note that individual studies defined and measured prognostic factors differently. A salient example is family history of glaucoma, a long-established risk factor for glaucoma conversion. ${ }^{40}$ Family history of glaucoma is an unlikely prognostic factor for visual field progression in our review. In all 16 studies, it was simply defined as "family history of glaucoma." No information was available on how family history was determined. This is likely to be of importance, for example, with regard to the degree of familial relationship taken into account, the clinical course (i.e., disease progression) of affected family members, or severity of disease in terms of intervention types.

Our review treats the heterogeneity of study results qualitatively. For 19 presumed prognostic factors assessed under blind conditions we noted a considerable degree of heterogeneity across the study results and described inconsistencies in results for the five most important factors. A limitation of our review is that the analysis treated studies of different design and study quality equally. This is because a semi-quantitative approach gives fewer possibilities to differentiate between subtle study differences. Additionally, the studies used various covariates for adjustment in the multivariable analysis, which our evidence tables did not take into account. However, since univariate results generally resulted in the same associations as multivariable results, we feel that this effect had only a minor impact on our results.

Our aim is to present an overview of all studied prognostic factors, ranked according to their likelihood of being prognostic. We tried to include as many studies as possible so that this review might serve as a basis for further studies on prediction of glaucomatous visual field progression, for example, for the development of clinical prediction models or as an aid to select prognostic factors for further study. Application of clinical prediction models in practice would help clinicians to calculate the glaucoma patient's individual risk for clinically significant visual field progression. ${ }^{41}$ Using a risk calculator to estimate the risk of conversion in ocular hypertensive subjects has already been shown to change the treatment recommendations of glaucoma specialists. ${ }^{42}$ As long as there is no validated clinical prediction model for glaucoma progression, clinicians could use the most important prognostic factors elucidated in this review to make their own risk estimations. Information on these factors can be implicitly considered in decisions on treatment and monitoring strategies in individual patients. Other information, such as a patient's functional reserve and life expectancy, also plays a vital role in these decisions. 


\section{Clinical recommendations}

This review provides a database of all studied prognostic factors for glaucomatous visual field progression. We have summarized our results to translate them into usable, clinically relevant recommendations.

We consider two prognostic factors, found to be associated with glaucomatous visual field progression, very important for clinical practice: age for all OAG and disc hemorrhages for NTG. ${ }^{A}$,

The following six factors, ranked according to their strength of evidence, were found to be probably associated with glaucomatous visual field progression: baseline visual field loss, baseline IOP, exfoliation syndrome, CCT, peripapillary atrophy (for NTG), and proven previous visual field progression. ${ }^{A}$, "We consider them important for clinical practice, given that we feel there is a reasonable basis to evaluate these factors when deciding on subsequent treatment and monitoring strategies.

The following prognostic factors were found to be probably associated with visual field progression but are not prevalent or easy to assess in clinical practice: ocular blood flow resistivity index and blood anticardiolipin antibody for all OAG, and stroke for NTG. B, $^{\text {" }}$

Thirty factors were possibly associated with glaucomatous visual field progression. These factors have seldom been studied or showed inconsistent results between studies. Further research is needed to establish the relevance of these factors.

Most factors showed no evidence of an association with visual field progression, although the conclusion was based on a limited number of studies. Eight factors investigated in a high number of studies showed no evidence of an association. These unlikely prognostic factors are ranked according to the number of studies that investigated them: family history of glaucoma, atherosclerosis, systemic hypertension, visual acuity, gender (for NTG), systolic blood pressure, myopic refractive error (for NTG), and Raynaud's phenomenon. 


\section{References}

1. Kwon YH, Fingert JH, Kuehn MH, Alward WL. Primary open-angle glaucoma. N Engl J Med 2009;360:111324.

2. Chen PP. Blindness in patients with treated open-angle glaucoma. Ophthalmology 2003;110:726-33.

3. Hattenhauer MG, Johnson $\mathrm{DH}$, Ing $\mathrm{HH}$, et al. The probability of blindness from open-angle glaucoma. Ophthalmology 1998;105:2099-104.

4. Heijl A, Bengtsson B, Hyman L, Leske MC. Natural History of Open-Angle Glaucoma. Ophthalmology 2009;116:2271-6.

5. Grobbee DE, Hoes AW. Clinical epidemiology : principles, methods, and applications for clinical research. Sudbury, MA.: Jones and Bartlett Publishers; 2009; 104-6.

6. Coleman AL, Miglior S, Coleman AL, Miglior S. Risk factors for glaucoma onset and progression. Surv Ophthalmol 2008;53 Suppl1:S3-10.

7. Boland MV, Quigley HA, Boland MV, Quigley HA. Risk factors and open-angle glaucoma: classification and application. J Glaucoma 2007;16:406-18.

8. Kass MA, Kolker AE, Becker B, et al. Prognostic factors in glaucomatous visual field loss. Arch Ophthalmol 1976;94:1274-6.

9. Ernest PJ, Viechtbauer W, Schouten JS, et al. The influence of the assessment method on the incidence of visual field progression in glaucoma: a network meta-analysis. Acta Ophthalmol Scand 2012;90:10-9.

10. Ernest PJ, Schouten JS, Beckers HJ, et al. The evidence base to select a method for assessing glaucomatous visual field progression. Acta Ophthalmol Scand 2012;90:101-8.

11. Julvez LP, Del Castillo Sanchez JB, Feijoo JG, Rubio Terres C. Methodologic Quality of Studies on Prognostic Factors for Primary Open-angle Glaucoma Progression Measured by Visual Field Deterioration. J Glaucoma 2010;19:587-91.

12. Ophthalmology. Guide for Authors. Available at:

http://www.elsevier.com/framework_products/promis_misc/ophta_guideforauthors2012.pdf. Accessed August 1, 2012.

13. Hayden JA, Cote P, Bombardier C. Evaluation of the quality of prognosis studies in systematic reviews. Ann Intern Med 2006;144:427-37.

14. Wesselink C, Marcus MW, Jansonius NM. Risk Factors for Visual Field Progression in the Groningen Longitudinal Glaucoma Study: A Comparison of Different Statistical Approaches. J Glaucoma 2012;21:57985.

15. Deeks JJ, Higgins JP, Altman DG, Cochrane Statistical Methods Group, eds. Chapter 9.5.1: What is heterogeneity? In: Higgins JPT, Green S, eds. Cochrane Handbook for Systematic Reviews of Interventions 5.1.0 [updated March 2011]. The Cochrane Collaboration; 2011. Available at: www.cochranehandbook.org. Accessed August 28, 2012.

16. Olthoff CM, Schouten JS, van de Borne BW, et al. Noncompliance with ocular hypotensive treatment in patients with glaucoma or ocular hypertension an evidence-based review. Ophthalmology 2005;112:95361.

17. Cohen J. Weighted kappa: nominal scale agreement with provision for scaled disagreement or partial credit. Psychol Bull 1968;70:213-20.

18. Fleiss JL, Cohen J. The equivalence of weighted kappa and the intraclass correlation coefficient as measures of reliability. Educ Psychol Meas 1973;33:613-9.

19. Wilson MR. Progression of visual field loss in untreated glaucoma patients and suspects in St Lucia, West Indies. Trans Am Ophthalmol Soc 2002;100:365-410.

20. Kim JW, Chen PP. Central corneal pachymetry and visual field progression in patients with open-angle glaucoma. Ophthalmology 2004;111:2126-32. 
21. Schwartz B, Takamoto T, Martin J. Increased Rate of Visual Field Loss Associated with Larger Initial Visual Field Threshold Values on Follow-Up of Open-Angle Glaucoma. J Glaucoma 2004;13:120-9.

22. Nouri Mahdavi K, Hoffman D, Coleman AL, et al. Predictive factors for glaucomatous visual field progression in the Advanced Glaucoma Intervention Study. Ophthalmology 2004;111:1627-35.

23. Daugeliene L, Yamamoto T, Kitazawa Y. Risk factors for visual field damage progression in normal-tension glaucoma eyes. Graefes Arch Clin Exp Ophthalmol 1999;237:105-8.

24. Blackwell B, Gaasterland D, Ederer F, et al. The Advanced Glaucoma Intervention Study (AGIS): 12. Baseline risk factors for sustained loss of visual field and visual acuity in patients with advanced glaucoma. Am J Ophthalmol 2002;134:499-512.

25. Caprioli J, Coleman AL. Intraocular Pressure Fluctuation A Risk Factor for Visual Field Progression at Low Intraocular Pressures in the Advanced Glaucoma Intervention Study. Ophthalmology 2008;115:1123-9.

26. Prata TS, De Moraes CGV, Teng CC, et al. Factors Affecting Rates of Visual Field Progression in Glaucoma Patients with Optic Disc Hemorrhage. Ophthalmology 2010;117:24-9.

27. Martinez A, Sanchez M. Effects of dorzolamide $2 \%$ added to timolol maleate $0.5 \%$ on intraocular pressure, retrobulbar blood flow, and the progression of visual field damage in patients with primary open-angle glaucoma: A single-center, 4-year, open-label study. Clin Ther 2008;30:1120-34.

28. Ahrlich KG, De Moraes CG, Teng CC, et al. Visual field progression differences between normal-tension and exfoliative high-tension glaucoma. Invest Ophthalmol Vis Sci 2010;51:1458-63.

29. De Moraes CG, Prata TS, Tello C, et al. Glaucoma with early visual field loss affecting both hemifields and the risk of disease progression. Arch Ophthalmol 2009;127:1129-34.

30. Teng CC, De Moraes CGV, Prata TS, et al. beta-Zone Parapapillary Atrophy and the Velocity of Glaucoma Progression. Ophthalmology 2010;117:909-15.

31. Ishida K, Yamamoto T, Sugiyama K, Kitazawa Y. Disk hemorrhage is a significantly negative prognostic factor in normal-tension glaucoma. Am J Ophthalmol 2000;129:707-14.

32. Ishida K, Yamamoto T, Kitazawa Y. Clinical factors associated with progression of normal-tension glaucoma. J Glaucoma 1998;7:372-7.

33. Sawada A, Kitazawa Y, Yamamoto T, et al. Prevention of visual field defect progression with brovincamine in eyes with normal-tension glaucoma. Ophthalmology 1996;103:283-8.

34. Chauhan BC, Mikelberg FS, Balaszi AG, et al. Canadian Glaucoma Study: 2. risk factors for the progression of open-angle glaucoma. Arch Ophthalmol 2008;126:1030-6.

35. Musch DC, Gillespie BW, Lichter PR, et al. Visual Field Progression in the Collaborative Initial Glaucoma Treatment Study. The Impact of Treatment and Other Baseline Factors. Ophthalmology 2009;116:2007.e1.

36. Shields MB. Normal-tension glaucoma: is it different from primary open-angle glaucoma? Curr Opin Ophthalmol 2008;19:85-8.

37. Sommer A. Ocular hypertension and normal-tension glaucoma: time for banishment and burial. Arch Ophthalmol 2011;129:785-7.

38. Leung DYL, Tham CCY, Li FCH, et al. Silent Cerebral Infarct and Visual Field Progression in Newly Diagnosed Normal-Tension Glaucoma. A Cohort Study. Ophthalmology 2009;116:1250-6.

39. Leung DYL, Li FCH, Kwong YYY, et al. Simvastatin and Disease Stabilization in Normal Tension Glaucoma: A Cohort Study. Ophthalmology 2010;117:471-6.

40. Kass MA, Becker B. Genetics of primary open-angle glaucoma. Sight Sav Rev 1978;48:21-8.

41. Medeiros FA, Weinreb RN. Predictive models to estimate the risk of glaucoma development and progression. Prog Brain Res 2008;173:15-24.

42. Boland MV, Quigley HA, Lehmann HP. The impact of risk calculation on treatment recommendations made by glaucoma specialists in cases of ocular hypertension. J Glaucoma 2008;17:631-8. 


\section{Appendix}

Table 6: Evidence tables of prognostic factors for glaucomatous visual field progression

Evidence tables of definite prognostic factors for glaucomatous visual field progression

\begin{tabular}{|c|c|c|c|c|c|c|c|}
\hline \multirow[t]{2}{*}{ Older age (OAG) } & \multicolumn{3}{|c|}{ More progression } & \multirow[t]{2}{*}{ No relation } & \multicolumn{3}{|c|}{ Less progression } \\
\hline & Significant & & Non-significant & & $\begin{array}{c}\text { Non- } \\
\text { significant }\end{array}$ & & Significant \\
\hline $\begin{array}{l}\text { Univariate analysis } \\
(n=39)\end{array}$ & $\begin{array}{c}20, \underline{40,41} \\
\underline{60,61,63}, 69 \\
\underline{70,71}, \underline{80,81}\end{array}$ & $\begin{array}{c}2,56 \\
59\end{array}$ & $\begin{array}{l}6,12,18, \frac{36,37}{38,42,64,65} \\
\frac{66,67}{75}, \frac{72,73}{79}\end{array}$ & $\begin{array}{l}5,13,51 \\
58,82,84\end{array}$ & $7,44,49$ & & 9 \\
\hline No. of clusters & 6 & 3 & 12 & 6 & 3 & 0 & 1 \\
\hline No. of studies & 11 & 3 & 15 & 6 & 3 & 0 & 1 \\
\hline No. of patients & 2351 & 360 & 1710 & 454 & 377 & 0 & 282 \\
\hline $\begin{array}{l}\text { Multivariable analysis } \\
(n=26)\end{array}$ & $\begin{array}{l}3,17, \underline{38,40}, \frac{41}{55}, \frac{54,56}{57}, \underline{60,61}, 65 \\
69,70,81\end{array}$ & 62 & $64,67,80$ & $\begin{array}{l}13,28,36 \\
39,66,79\end{array}$ & 2 & & \\
\hline No. of clusters & 11 & 1 & 3 & 6 & 1 & 0 & 0 \\
\hline No. of studies & 15 & 1 & 3 & 6 & 1 & 0 & 0 \\
\hline No. of patients & 2430 & 255 & 400 & 997 & 57 & 0 & 0 \\
\hline
\end{tabular}

\begin{tabular}{|c|c|c|c|c|c|}
\hline \multirow{2}{*}{$\begin{array}{l}\text { Disc hemorrhages } \\
\text { (NTG) }\end{array}$} & \multicolumn{2}{|c|}{ More progression } & \multirow[t]{2}{*}{ No relation } & \multicolumn{2}{|c|}{ Less progression } \\
\hline & Significant & Non-significant & & $\begin{array}{c}\text { Non- } \\
\text { significant }\end{array}$ & Significant \\
\hline
\end{tabular}

Univariate analysis $24,30, \underline{76,77}$

52

$(n=5)$

No. of clusters

$\begin{array}{ccc}3 & 0 & 1 \\ 4 & 0 & 1 \\ 772 & 0 & 27\end{array}$

No. of patients

Multivariable analysis
$(n=6)$$\frac{21,23,24}{30,76,77}$

No. of clusters

$\begin{array}{ccc}3 & 0 & 0 \\ 6 & 0 & 0 \\ 851 & 0 & 0\end{array}$

No. of studies

No. of patients

851

0

These evidence tables summarize the univariate and multivariable results of prognostic factors. The tables were used to determine the extent to which prognostic factors were associated with glaucomatous visual field progression. The tables are ranked by the strength of the association between prognostic factor and visual field progression and the number of studies that investigated each factor (i.e., in the same order as in Tables 4 and 5). Each number in the tables represents a study and corresponds with the number in the reference list, which is the same as the reference list of Table 1 . Studies are classified in the tables by the direction and statistical significance $(P \leq 0.05)$ of the association.

Numbers of study clusters, studies, and patients are summarized in each table.

Numbers between parentheses represent the total number of studies in the analysis.

Underlined studies belong to the same study cluster and their results are likely to be correlated.

$\mathrm{OAG}=$ open-angle glaucoma, NTG = normal-tension glaucoma, IOP = intraocular pressure. 
Evidence tables of probable prognostic factors for glaucomatous visual field progression

\begin{tabular}{|c|c|c|c|c|c|c|c|}
\hline \multirow{2}{*}{$\begin{array}{l}\text { Baseline visual } \\
\text { field loss (OAG) }\end{array}$} & \multicolumn{3}{|c|}{ More progression } & \multirow[b]{2}{*}{ No relation } & \multicolumn{3}{|c|}{ Less progression } \\
\hline & Significant & & $\begin{array}{c}\text { Non- } \\
\text { significant }\end{array}$ & & $\begin{array}{c}\text { Non- } \\
\text { significant }\end{array}$ & & Significant \\
\hline $\begin{array}{l}\text { Univariate } \\
\text { analysis } \\
(n=47)\end{array}$ & $\begin{array}{c}5,7,9,40,60 \\
66,74^{*}, 81\end{array}$ & $\begin{array}{c}2,37 \\
79\end{array}$ & $\begin{array}{l}10^{*}, 20,35^{*}, \\
36,38, \underline{42}, \\
\frac{44}{49}, \frac{47,48,}{50}, 61, \\
\frac{49,73,75,}{66^{*}, 77^{*}} \\
\frac{82}{80,}\end{array}$ & $\begin{array}{c}11,19,26^{*} \\
30^{*}, 32^{*}, 64 \\
65,51,59,72 \\
84\end{array}$ & $\underline{12,13}, 41$ & $34^{*}, 56$ & 70 \\
\hline No. of clusters & 8 & 3 & 13 & 11 & 2 & 2 & 1 \\
\hline No. of studies & 8 & 3 & 19 & 11 & 3 & 2 & 1 \\
\hline No. of patients & 1188 & 513 & 3021 & 1072 & 471 & 191 & 258 \\
\hline $\begin{array}{l}\text { Multivariable } \\
\text { analysis } \\
(n=36)\end{array}$ & $\begin{array}{c}\underline{2,3}, 35^{*}, 48 \\
57,60,66 \\
\underline{79}, 81,85\end{array}$ & $\frac{\frac{22^{*}}{24^{*}, 54}}{72,74^{*}}$ & $\frac{38,40}{61,64,87}$ & $\begin{array}{c}16^{*}, 17,21^{*} \\
28,30^{*}, 34^{*} \\
43,49,62\end{array}$ & $23 *, 50$ & $13,41,56$ & 39 \\
\hline No. of clusters & 8 & 4 & 5 & 9 & 2 & 3 & 1 \\
\hline No. of studies & 10 & 5 & 6 & 9 & 2 & 3 & 1 \\
\hline No. of patients & 1874 & 928 & 1059 & 930 & 135 & 586 & 558 \\
\hline \multirow{2}{*}{$\begin{array}{l}\text { Baseline IOP } \\
\text { (OAG) }\end{array}$} & \multicolumn{3}{|c|}{ More progression } & & \multicolumn{3}{|c|}{ Less progression } \\
\hline & Significant & & $\begin{array}{c}\text { Non- } \\
\text { significant }\end{array}$ & No relation & $\begin{array}{c}\text { Non- } \\
\text { significant }\end{array}$ & & Significant \\
\hline $\begin{array}{l}\text { Univariate } \\
\text { analysis } \\
(n=22)\end{array}$ & $\underline{60,61}, \underline{79,82}$ & & $\begin{array}{c}7,36, \underline{38,40} \\
58,59,73\end{array}$ & $9,11,70$ & $\begin{array}{c}2,41,49,64 \\
66,75,81\end{array}$ & 72 & \\
\hline No. of clusters & 2 & 0 & 6 & 3 & 7 & 1 & 0 \\
\hline No. of studies & 4 & 0 & 7 & 3 & 7 & 1 & 0 \\
\hline No. of patients & 960 & 0 & 1039 & 717 & 971 & 40 & 0 \\
\hline $\begin{array}{l}\text { Multivariable } \\
\text { analysis } \\
(n=17)\end{array}$ & $\begin{array}{c}2,51,61,79 \\
85\end{array}$ & 60 & 40,64 & $\begin{array}{c}17,28,39,62 \\
66,72,82\end{array}$ & $\underline{38,41}$ & & \\
\hline No. of clusters & 5 & 1 & 2 & 7 & 1 & 0 & 0 \\
\hline No. of studies & 5 & 1 & 2 & 7 & 2 & 0 & 0 \\
\hline No. of patients & 905 & 255 & 210 & 1439 & 702 & 0 & 0 \\
\hline
\end{tabular}

*Studies comprising a majority of normal-tension glaucoma patients. 


\begin{tabular}{|c|c|c|c|c|c|c|c|}
\hline \multirow{2}{*}{$\begin{array}{l}\text { Exfoliation } \\
\text { syndrome (OAG) }\end{array}$} & \multicolumn{3}{|c|}{ More progression } & \multirow[b]{2}{*}{ No relation } & \multicolumn{3}{|c|}{ Less progression } \\
\hline & Significant & & $\begin{array}{c}\text { Non- } \\
\text { significant }\end{array}$ & & $\begin{array}{c}\text { Non- } \\
\text { significant }\end{array}$ & & Significant \\
\hline $\begin{array}{l}\text { Univariate } \\
\text { analysis } \\
(n=11)\end{array}$ & $47, \underline{60,61,63}$ & 80 & $\begin{array}{l}12,48,66 \\
70, \underline{79,81}\end{array}$ & & & & \\
\hline No. of clusters & 2 & 1 & 5 & 0 & 0 & 0 & 0 \\
\hline No. of studies & 4 & 1 & 6 & 0 & 0 & 0 & 0 \\
\hline No. of patients & 676 & 293 & 802 & 0 & 0 & 0 & 0 \\
\hline $\begin{array}{l}\text { Multivariable } \\
\text { analysis }(n=6)\end{array}$ & $\underline{60,61,62}$ & & 80 & 47,66 & & & \\
\hline No. of clusters & 1 & 0 & 1 & 2 & 0 & 0 & 0 \\
\hline No. of studies & 3 & 0 & 1 & 2 & 0 & 0 & 0 \\
\hline No. of patients & 765 & 0 & 293 & 119 & 0 & 0 & 0 \\
\hline
\end{tabular}

\begin{tabular}{|c|c|c|c|c|c|c|c|}
\hline \multirow{2}{*}{$\begin{array}{l}\text { Thinner central } \\
\text { corneal thickness } \\
\text { (OAG) }\end{array}$} & \multicolumn{3}{|c|}{ More progression } & \multirow[b]{2}{*}{ No relation } & \multicolumn{3}{|c|}{ Less progression } \\
\hline & Significant & & $\begin{array}{c}\text { Non- } \\
\text { significant }\end{array}$ & & $\begin{array}{c}\text { Non- } \\
\text { significant }\end{array}$ & & Significant \\
\hline $\begin{array}{l}\text { Univariate } \\
\text { analysis } \\
(n=5)\end{array}$ & $\underline{79,82}$ & & 80 & & 72,81 & & \\
\hline No. of clusters & 1 & 0 & 1 & 0 & 2 & 0 & 0 \\
\hline No. of studies & 2 & 0 & 1 & 0 & 2 & 0 & 0 \\
\hline No. of patients & 450 & 0 & 293 & 0 & 116 & 0 & 0 \\
\hline $\begin{array}{l}\text { Multivariable } \\
\text { analysis } \\
(n=3)\end{array}$ & $\underline{79,80,82}$ & & & & & & \\
\hline No. of clusters & 1 & 0 & 0 & 0 & 0 & 0 & 0 \\
\hline No. of studies & 3 & 0 & 0 & 0 & 0 & 0 & 0 \\
\hline No. of patients & 743 & 0 & 0 & 0 & 0 & 0 & 0 \\
\hline
\end{tabular}

\begin{tabular}{|c|c|c|c|c|c|c|c|}
\hline \multirow{2}{*}{$\begin{array}{l}\text { Ocular blood flow } \\
\text { resistivity index } \\
\text { (OAG) }\end{array}$} & \multicolumn{3}{|c|}{ More progression } & \multirow[b]{2}{*}{ No relation } & \multicolumn{3}{|c|}{ Less progression } \\
\hline & Significant & & $\begin{array}{c}\text { Non- } \\
\text { significant }\end{array}$ & & $\begin{array}{c}\text { Non- } \\
\text { significant }\end{array}$ & & Significant \\
\hline $\begin{array}{l}\text { Univariate } \\
\text { analysis }(n=3)\end{array}$ & $\begin{array}{c}\frac{64^{\ddagger}, 64^{5}}{72^{\ddagger}, 73^{7}, 73^{5}} \\
\underline{4}\end{array}$ & & $73^{+}$ & & & $\underline{72^{\dagger}, 72^{\S}}$ & \\
\hline No. of clusters & 2 & 0 & 1 & 0 & 0 & 1 & 0 \\
\hline No. of studies & 3 & 0 & 1 & 0 & 0 & 1 & 0 \\
\hline No. of patients & 235 & 0 & 146 & 0 & 0 & 40 & 0 \\
\hline $\begin{array}{l}\text { Multivariable } \\
\text { analysis }(n=3)\end{array}$ & $\begin{array}{c}\underline{64^{\ddagger}, 64^{5}} \\
\underline{72^{\ddagger}, 73^{7}, 73^{8}}\end{array}$ & & & $\underline{72^{\dagger}, 72^{\S}}$ & & & \\
\hline No. of clusters & 2 & 0 & 0 & 1 & 0 & 0 & 0 \\
\hline No. of studies & 3 & 0 & 0 & 1 & 0 & 0 & 0 \\
\hline No. of patients & 235 & 0 & 0 & 40 & 0 & 0 & 0 \\
\hline
\end{tabular}

†In central retinal artery; ¥In ophthalmic artery; §In short posterior ciliary arteries. 


\begin{tabular}{|c|c|c|c|c|c|c|c|}
\hline \multirow{2}{*}{$\begin{array}{l}\text { Anticardiolipin } \\
\text { antibody in blood } \\
\text { (OAG) }\end{array}$} & \multicolumn{3}{|c|}{ More progression } & \multirow[b]{2}{*}{ No relation } & \multicolumn{3}{|c|}{ Less progression } \\
\hline & \multicolumn{2}{|l|}{ Significant } & $\begin{array}{c}\text { Non- } \\
\text { significant }\end{array}$ & & $\begin{array}{c}\text { Non- } \\
\text { significant }\end{array}$ & & Significant \\
\hline $\begin{array}{l}\text { Univariate } \\
\text { analysis }(n=2)\end{array}$ & \multicolumn{3}{|l|}{$\underline{70,71}$} & & & & \\
\hline No. of clusters & 1 & 0 & 0 & 0 & 0 & 0 & 0 \\
\hline No. of studies & 2 & 0 & 0 & 0 & 0 & 0 & 0 \\
\hline No. of patients & 474 & 0 & 0 & 0 & 0 & 0 & 0 \\
\hline $\begin{array}{l}\text { Multivariable } \\
\text { analysis }(n=1)\end{array}$ & 70 & & & & & & \\
\hline No. of clusters & 1 & 0 & 0 & 0 & 0 & 0 & 0 \\
\hline No. of studies & 1 & 0 & 0 & 0 & 0 & 0 & 0 \\
\hline No. of patients & 258 & 0 & 0 & 0 & 0 & 0 & 0 \\
\hline \multirow{2}{*}{$\begin{array}{l}\text { Peripapillary } \\
\text { atrophy (NTG) }\end{array}$} & \multicolumn{3}{|c|}{ More progression } & & \multicolumn{3}{|c|}{ Less progression } \\
\hline & Significant & & $\begin{array}{c}\text { Non- } \\
\text { significant }\end{array}$ & No relation & $\begin{array}{c}\text { Non- } \\
\text { significant }\end{array}$ & & Significant \\
\hline \multicolumn{8}{|l|}{$\begin{array}{l}\text { Univariate } \\
\text { analysis }(n=0)\end{array}$} \\
\hline No. of clusters & 0 & 0 & 0 & 0 & 0 & 0 & 0 \\
\hline No. of studies & 0 & 0 & 0 & 0 & 0 & 0 & 0 \\
\hline No. of patients & 0 & 0 & 0 & 0 & 0 & 0 & 0 \\
\hline $\begin{array}{l}\text { Multivariable } \\
\text { analysis }(n=2)\end{array}$ & 16,23 & & & & & & \\
\hline No. of clusters & 2 & 0 & 0 & 0 & 0 & 0 & 0 \\
\hline No. of studies & 2 & 0 & 0 & 0 & 0 & 0 & 0 \\
\hline No. of patients & 103 & 0 & 0 & 0 & 0 & 0 & 0 \\
\hline \multirow{2}{*}{$\begin{array}{l}\text { Previous visual } \\
\text { field progression } \\
\text { (OAG) }\end{array}$} & \multicolumn{3}{|c|}{ More progression } & & \multicolumn{3}{|c|}{ Less progression } \\
\hline & Significant & & $\begin{array}{c}\text { Non- } \\
\text { significant }\end{array}$ & No relation & $\begin{array}{c}\text { Non- } \\
\text { significant }\end{array}$ & & Significant \\
\hline $\begin{array}{l}\text { Univariate } \\
\text { analysis }(n=2)\end{array}$ & 40 & 46 & & & & & \\
\hline No. of clusters & 1 & 1 & 0 & 0 & 0 & 0 & 0 \\
\hline No. of studies & 1 & 1 & 0 & 0 & 0 & 0 & 0 \\
\hline No. of patients & 161 & 81 & 0 & 0 & 0 & 0 & 0 \\
\hline $\begin{array}{l}\text { Multivariable } \\
\text { analysis }(n=1)\end{array}$ & 40 & & & & & & \\
\hline No. of clusters & 1 & 0 & 0 & 0 & 0 & 0 & 0 \\
\hline No. of studies & 1 & 0 & 0 & 0 & 0 & 0 & 0 \\
\hline No. of patients & 161 & 0 & 0 & 0 & 0 & 0 & 0 \\
\hline
\end{tabular}




\begin{tabular}{|c|c|c|c|c|c|c|c|}
\hline \multirow[b]{2}{*}{ Stroke (NTG) } & \multicolumn{3}{|c|}{ More progression } & \multirow[b]{2}{*}{ No relation } & \multicolumn{3}{|c|}{ Less progression } \\
\hline & Significant & & $\begin{array}{c}\text { Non- } \\
\text { significant }\end{array}$ & & $\begin{array}{c}\text { Non- } \\
\text { significant }\end{array}$ & & Significant \\
\hline $\begin{array}{l}\text { Univariate } \\
\text { analysis }(n=2)\end{array}$ & 76 & & 77 & & & & \\
\hline No. of clusters & 1 & 0 & 1 & 0 & 0 & 0 & 0 \\
\hline No. of studies & 1 & 0 & 1 & 0 & 0 & 0 & 0 \\
\hline No. of patients & 286 & 0 & 256 & 0 & 0 & 0 & 0 \\
\hline $\begin{array}{l}\text { Multivariable } \\
\text { analysis }(n=2)\end{array}$ & $\underline{76,77}$ & & & & & & \\
\hline No. of clusters & 1 & 0 & 0 & 0 & 0 & 0 & 0 \\
\hline No. of studies & 2 & 0 & 0 & 0 & 0 & 0 & 0 \\
\hline No. of patients & 542 & 0 & 0 & 0 & 0 & 0 & 0 \\
\hline
\end{tabular}


Evidence tables of possible prognostic factors for glaucomatous visual field progression

\begin{tabular}{|c|c|c|c|c|c|c|c|}
\hline \multirow[b]{2}{*}{ Female sex (OAG) } & \multicolumn{3}{|c|}{ More progression } & \multirow[b]{2}{*}{ No relation } & \multicolumn{3}{|c|}{ Less progression } \\
\hline & Significant & & $\begin{array}{c}\text { Non- } \\
\text { significant }\end{array}$ & & $\begin{array}{c}\text { Non- } \\
\text { significant }\end{array}$ & & Significant \\
\hline $\begin{array}{l}\text { Univariate } \\
\text { analysis }(n=23)\end{array}$ & 70 & 2 & $\begin{array}{l}6,12,20,36 \\
40,60, \underline{72,73}\end{array}$ & $9,51,61,71$ & $\begin{array}{l}18, \frac{38,41}{44,66}, \\
\frac{42,44,82}{\underline{81}}\end{array}$ & & 65 \\
\hline No. of clusters & 1 & 1 & 7 & 4 & 5 & 0 & 1 \\
\hline No. of studies & 1 & 1 & 8 & 4 & 8 & 0 & 1 \\
\hline No. of patients & 258 & 57 & 762 & 793 & 1334 & 0 & 108 \\
\hline $\begin{array}{l}\text { Multivariable } \\
\text { analysis }(n=17)\end{array}$ & 2,70 & & 57 & $\begin{array}{r}3,13,36 \\
54,55,56 \\
60,62,66,72\end{array}$ & $\underline{38,41}, 65$ & 39 & \\
\hline No. of clusters & 2 & 0 & 1 & 8 & 2 & 1 & 0 \\
\hline No. of studies & 2 & 0 & 1 & 10 & 3 & 1 & 0 \\
\hline No. of patients & 315 & 0 & 135 & 2534 & 810 & 558 & 0 \\
\hline \multirow{2}{*}{$\begin{array}{l}\text { Cup disc ratio } \\
\text { (OAG) }\end{array}$} & \multicolumn{3}{|c|}{ More progression } & & \multicolumn{3}{|c|}{ Less progression } \\
\hline & Significant & & $\begin{array}{c}\text { Non- } \\
\text { significant }\end{array}$ & No relation & $\begin{array}{c}\text { Non- } \\
\text { significant }\end{array}$ & & Significant \\
\hline $\begin{array}{l}\text { Univariate } \\
\text { analysis }(n=14)\end{array}$ & $7,47,69$ & & $\begin{array}{l}41, \underline{49,50} \\
\underline{76^{*}, 77^{*}}\end{array}$ & $\begin{array}{c}9,20,30 *, 48 \\
51,65\end{array}$ & & & \\
\hline No. of clusters & 3 & 0 & 3 & 6 & 0 & 0 & 0 \\
\hline No. of studies & 3 & 0 & 5 & 6 & 0 & 0 & 0 \\
\hline No. of patients & 507 & 0 & 1098 & 798 & 0 & 0 & 0 \\
\hline $\begin{array}{l}\text { Multivariable } \\
\text { analysis }(n=7)\end{array}$ & $16^{*}, 69$ & & 38,50 & $17,41,47$ & & & \\
\hline No. of clusters & 2 & 0 & 2 & 3 & 0 & 0 & 0 \\
\hline No. of studies & 2 & 0 & 2 & 3 & 0 & 0 & 0 \\
\hline No. of patients & 318 & 0 & 389 & 617 & 0 & 0 & 0 \\
\hline
\end{tabular}

*Studies comprising a majority of normal-tension glaucoma patients. 


\begin{tabular}{|c|c|c|c|c|c|c|c|}
\hline \multirow[b]{2}{*}{ Older age (NTG) } & \multicolumn{3}{|c|}{ More progression } & \multirow[b]{2}{*}{ No relation } & \multicolumn{3}{|c|}{ Less progression } \\
\hline & Significant & & $\begin{array}{c}\text { Non- } \\
\text { significant }\end{array}$ & & $\begin{array}{c}\text { Non- } \\
\text { significant }\end{array}$ & & Significant \\
\hline $\begin{array}{l}\text { Univariate } \\
\text { analysis }(n=10)\end{array}$ & $\underline{76,77}$ & & $\begin{array}{c}26,32 \\
34,35,74\end{array}$ & 10,30 & 52 & & \\
\hline No. of clusters & 1 & 0 & 4 & 2 & 1 & 0 & 0 \\
\hline No. of studies & 2 & 0 & 5 & 2 & 1 & 0 & 0 \\
\hline No. of patients & 542 & 0 & 349 & 196 & 27 & 0 & 0 \\
\hline $\begin{array}{l}\text { Multivariable } \\
\text { analysis }(n=11)\end{array}$ & 77 & 24 & $\underline{21,23}, 83$ & $\begin{array}{l}16,22,30 \\
34,35,76\end{array}$ & & & \\
\hline No. of clusters & 1 & 1 & 2 & 5 & 0 & 0 & 0 \\
\hline No. of studies & 1 & 1 & 3 & 6 & 0 & 0 & 0 \\
\hline No. of patients & 256 & 70 & 222 & 740 & 0 & 0 & 0 \\
\hline
\end{tabular}

\begin{tabular}{|c|c|c|c|c|c|c|c|}
\hline \multirow{2}{*}{$\begin{array}{l}\text { Myopic refractive } \\
\text { error (OAG) }\end{array}$} & \multicolumn{3}{|c|}{ More progression } & \multirow[b]{2}{*}{ No relation } & \multicolumn{3}{|c|}{ Less progression } \\
\hline & Significant & & $\begin{array}{c}\text { Non- } \\
\text { significant }\end{array}$ & & $\begin{array}{c}\text { Non- } \\
\text { significant }\end{array}$ & & Significant \\
\hline $\begin{array}{l}\text { Univariate } \\
\text { analysis }(n=12)\end{array}$ & 68,69 & & $50,60,82$ & $9,13,51,61,65$ & 12,41 & & \\
\hline No. of clusters & 2 & 0 & 3 & 5 & 2 & 0 & 0 \\
\hline No. of studies & 2 & 0 & 3 & 5 & 2 & 0 & 0 \\
\hline No. of patients & 372 & 0 & 588 & 715 & 441 & 0 & 0 \\
\hline $\begin{array}{l}\text { Multivariable } \\
\text { analysis } \\
(n=10)\end{array}$ & 69 & 13 & $38,50,57$ & $\underline{39}, 41, \underline{60,62}$ & 82 & & \\
\hline No. of clusters & 1 & 1 & 3 & 2 & 1 & 0 & 0 \\
\hline No. of studies & 1 & 1 & 3 & 4 & 1 & 0 & 0 \\
\hline No. of patients & 262 & 30 & 524 & 1469 & 245 & 0 & 0 \\
\hline
\end{tabular}

\begin{tabular}{|c|c|c|c|c|c|c|c|}
\hline \multirow[b]{2}{*}{ Diabetes (OAG) } & \multicolumn{3}{|c|}{ More progression } & \multirow[b]{2}{*}{ No relation } & \multicolumn{3}{|c|}{ Less progression } \\
\hline & Significant & & $\begin{array}{l}\text { Non- } \\
\text { significant }\end{array}$ & & $\begin{array}{c}\text { Non- } \\
\text { significant }\end{array}$ & & Significant \\
\hline $\begin{array}{l}\text { Univariate } \\
\text { analysis }(n=9)\end{array}$ & & & 38,70 & $4,9,27,65$ & $12,41,42$ & & \\
\hline No. of clusters & 0 & 0 & 2 & 4 & 3 & 0 & 0 \\
\hline No. of studies & 0 & 0 & 2 & 4 & 3 & 0 & 0 \\
\hline No. of patients & 0 & 0 & 559 & 486 & 485 & 0 & 0 \\
\hline $\begin{array}{l}\text { Multivariable } \\
\text { analysis }(n=5)\end{array}$ & 54 & 39 & & 57 & $\underline{38,41}$ & & \\
\hline No. of clusters & 1 & 1 & 0 & 1 & 1 & 0 & 0 \\
\hline No. of studies & 1 & 1 & 0 & 1 & 2 & 0 & 0 \\
\hline No. of patients & 607 & 558 & 0 & 135 & 702 & 0 & 0 \\
\hline
\end{tabular}




\begin{tabular}{|c|c|c|c|c|c|c|c|}
\hline \multirow{2}{*}{$\begin{array}{l}\text { African descent } \\
\text { (OAG) }\end{array}$} & \multicolumn{3}{|c|}{ More progression } & \multirow[b]{2}{*}{ No relation } & \multicolumn{3}{|c|}{ Less progression } \\
\hline & Significant & & $\begin{array}{c}\text { Non- } \\
\text { significant }\end{array}$ & & $\begin{array}{l}\text { Non- } \\
\text { significant }\end{array}$ & & Significant \\
\hline $\begin{array}{l}\text { Univariate } \\
\text { analysis }(n=7)\end{array}$ & & & $12,20, \underline{38,40}$ & $13,41,48$ & & & \\
\hline No. of clusters & 0 & 0 & 3 & 3 & 0 & 0 & 0 \\
\hline No. of studies & 0 & 0 & 4 & 3 & 0 & 0 & 0 \\
\hline No. of patients & 0 & 0 & 558 & 583 & 0 & 0 & 0 \\
\hline $\begin{array}{l}\text { Multivariable } \\
\text { analysis }(n=5)\end{array}$ & 13 & 54 & 38 & $\underline{39,41}$ & & & \\
\hline No. of clusters & 1 & 1 & 1 & 1 & 0 & 0 & 0 \\
\hline No. of studies & 1 & 1 & 1 & 2 & 0 & 0 & 0 \\
\hline No. of patients & 30 & 607 & 301 & 959 & 0 & 0 & 0 \\
\hline \multirow{2}{*}{$\begin{array}{l}\text { Baseline untreat- } \\
\text { ed IOP (OAG) }\end{array}$} & \multicolumn{3}{|c|}{ More progression } & & \multicolumn{3}{|c|}{ Less progression } \\
\hline & Significant & & $\begin{array}{c}\text { Non- } \\
\text { significant }\end{array}$ & No relation & $\begin{array}{c}\text { Non- } \\
\text { significant }\end{array}$ & & Significant \\
\hline $\begin{array}{l}\text { Univariate } \\
\text { analysis }(n=6)\end{array}$ & $\underline{60,61}$ & & 7,59 & 70 & 64 & & \\
\hline No. of clusters & 1 & 0 & 2 & 1 & 1 & 0 & 0 \\
\hline No. of studies & 2 & 0 & 2 & 1 & 1 & 0 & 0 \\
\hline No. of patients & 510 & 0 & 345 & 258 & 49 & 0 & 0 \\
\hline $\begin{array}{l}\text { Multivariable } \\
\text { analysis }(n=6)\end{array}$ & 61 & 60 & 64 & $17,28,62$ & & & \\
\hline No. of clusters & 1 & 1 & 1 & 3 & 0 & 0 & 0 \\
\hline No. of studies & 1 & 1 & 1 & 3 & 0 & 0 & 0 \\
\hline No. of patients & 255 & 255 & 49 & 525 & 0 & 0 & 0 \\
\hline
\end{tabular}

\begin{tabular}{|c|c|c|c|c|c|c|c|}
\hline \multirow{2}{*}{$\begin{array}{l}\text { Disc hemorrhages } \\
\text { (OAG) }\end{array}$} & \multicolumn{3}{|c|}{ More progression } & \multirow[b]{2}{*}{ No relation } & \multicolumn{3}{|c|}{ Less progression } \\
\hline & Significant & & $\begin{array}{c}\text { Non- } \\
\text { significant }\end{array}$ & & $\begin{array}{c}\text { Non- } \\
\text { significant }\end{array}$ & & Significant \\
\hline $\begin{array}{l}\text { Univariate } \\
\text { analysis }(n=7)\end{array}$ & $1,15,27,78$ & & $12,50,60$ & & & & \\
\hline No. of clusters & 4 & 0 & 3 & 0 & 0 & 0 & 0 \\
\hline No. of studies & 4 & 0 & 3 & 0 & 0 & 0 & 0 \\
\hline No. of patients & 352 & 0 & 383 & 0 & 0 & 0 & 0 \\
\hline $\begin{array}{l}\text { Multivariable } \\
\text { analysis }(n=3)\end{array}$ & & & 50 & 55,60 & & & \\
\hline No. of clusters & 0 & 0 & 1 & 2 & 0 & 0 & 0 \\
\hline No. of studies & 0 & 0 & 1 & 2 & 0 & 0 & 0 \\
\hline No. of patients & 0 & 0 & 88 & 862 & 0 & 0 & 0 \\
\hline
\end{tabular}




\begin{tabular}{|c|c|c|c|c|c|c|c|}
\hline \multirow{2}{*}{$\begin{array}{l}\text { Diastolic blood } \\
\text { pressure (OAG) }\end{array}$} & \multicolumn{3}{|c|}{ More progression } & \multirow[b]{2}{*}{ No relation } & \multicolumn{3}{|c|}{ Less progression } \\
\hline & Significant & & $\begin{array}{c}\text { Non- } \\
\text { significant }\end{array}$ & & $\begin{array}{c}\text { Non- } \\
\text { significant }\end{array}$ & & Significant \\
\hline $\begin{array}{l}\text { Univariate } \\
\text { analysis }(n=5)\end{array}$ & & & 70 & 61 & 60,64 & & 73 \\
\hline No. of clusters & 0 & 0 & 1 & 1 & 2 & 0 & 1 \\
\hline No. of studies & 0 & 0 & 1 & 1 & 2 & 0 & 1 \\
\hline No. of patients & 0 & 0 & 258 & 255 & 304 & 0 & 146 \\
\hline $\begin{array}{l}\text { Multivariable } \\
\text { analysis }(n=4)\end{array}$ & & & 64 & $\underline{60,62}$ & & & 73 \\
\hline No. of clusters & 0 & 0 & 1 & 1 & 0 & 0 & 1 \\
\hline No. of studies & 0 & 0 & 1 & 2 & 0 & 0 & 1 \\
\hline No. of patients & 0 & 0 & 49 & 510 & 0 & 0 & 146 \\
\hline
\end{tabular}

\begin{tabular}{|c|c|c|c|c|c|c|c|}
\hline \multirow{2}{*}{$\begin{array}{l}\text { Systemic hyper- } \\
\text { tension (NTG) }\end{array}$} & \multicolumn{3}{|c|}{ More progression } & \multirow[b]{2}{*}{ No relation } & \multicolumn{3}{|c|}{ Less progression } \\
\hline & & & $\begin{array}{c}\text { Non- } \\
\text { significant }\end{array}$ & & & & Significant \\
\hline $\begin{array}{l}\text { Univariate } \\
\text { analysis }(n=4)\end{array}$ & 76 & & 77 & 30 & 26 & & \\
\hline No. of clusters & 1 & 0 & 1 & 1 & 1 & 0 & 0 \\
\hline No. of studies & 1 & 0 & 1 & 1 & 1 & 0 & 0 \\
\hline No. of patients & 286 & 0 & 256 & 160 & 59 & 0 & 0 \\
\hline $\begin{array}{l}\text { Multivariable } \\
\text { analysis }(n=4)\end{array}$ & 76 & & & $\underline{22,24}, 30$ & & & \\
\hline No. of clusters & 1 & 0 & 0 & 2 & 0 & 0 & 0 \\
\hline No. of studies & 1 & 0 & 0 & 3 & 0 & 0 & 0 \\
\hline No. of patients & 286 & 0 & 0 & 340 & 0 & 0 & 0 \\
\hline
\end{tabular}

\begin{tabular}{|c|c|c|c|c|c|}
\hline \multirow{2}{*}{$\begin{array}{l}\text { Recovery rate } \\
\text { from cold } \\
\text { exposure test } \\
\text { (NTG) }\end{array}$} & \multicolumn{2}{|c|}{ More progression } & \multirow[b]{2}{*}{ No relation } & \multicolumn{2}{|c|}{ Less progression } \\
\hline & Significant & $\begin{array}{c}\text { Non- } \\
\text { significant }\end{array}$ & & $\begin{array}{c}\text { Non- } \\
\text { significant }\end{array}$ & Significant \\
\hline
\end{tabular}

Univariate

analysis ( $n=0)$

\begin{tabular}{llllcccc} 
No. of clusters & 0 & 0 & 0 & 0 & 0 & 0 & 0 \\
No. of studies & 0 & 0 & 0 & 0 & 0 & 0 & 0 \\
$\begin{array}{l}\text { No. of patients } \\
\text { Multivariable }\end{array}$ & 0 & 0 & 0 & 0 & 0 & 0 & 0 \\
$\begin{array}{l}\text { analysis }(n=5) \\
\text { No. of clusters }\end{array}$ & 0 & 0 & 0 & $\underline{21,24}$ & 23 & 22 & 25 \\
No. of studies & 0 & 0 & 0 & 1 & 1 & 1 & 1 \\
No. of patients & 0 & 0 & 0 & 102 & 47 & 110 & 28 \\
\hline
\end{tabular}




\begin{tabular}{|c|c|c|c|c|c|c|c|}
\hline \multirow{2}{*}{$\begin{array}{l}\text { Baseline IOP } \\
\text { fluctuation (OAG) }\end{array}$} & \multicolumn{3}{|c|}{ More progression } & \multirow[b]{2}{*}{ No relation } & \multicolumn{3}{|c|}{ Less progression } \\
\hline & Significant & & $\begin{array}{c}\text { Non- } \\
\text { significant }\end{array}$ & & $\begin{array}{c}\text { Non- } \\
\text { significant }\end{array}$ & & Significant \\
\hline $\begin{array}{l}\text { Univariate } \\
\text { analysis }(n=3)\end{array}$ & & & $40, \underline{72,73}$ & & & & \\
\hline No. of clusters & 0 & 0 & 2 & 0 & 0 & 0 & 0 \\
\hline No. of studies & 0 & 0 & 3 & 0 & 0 & 0 & 0 \\
\hline No. of patients & 0 & 0 & 347 & 0 & 0 & 0 & 0 \\
\hline $\begin{array}{l}\text { Multivariable } \\
\text { analysis }(n=2)\end{array}$ & 55 & & & & 40 & & \\
\hline No. of clusters & 1 & 0 & 0 & 0 & 1 & 0 & 0 \\
\hline No. of studies & 1 & 0 & 0 & 0 & 1 & 0 & 0 \\
\hline No. of patients & 607 & 0 & 0 & 0 & 161 & 0 & 0 \\
\hline
\end{tabular}

\begin{tabular}{|c|c|c|c|c|c|c|c|}
\hline \multirow[b]{2}{*}{ Migraine (NTG) } & \multicolumn{3}{|c|}{ More progression } & \multirow[b]{2}{*}{ No relation } & \multicolumn{3}{|c|}{ Less progression } \\
\hline & Significant & & $\begin{array}{c}\text { Non- } \\
\text { significant }\end{array}$ & & $\begin{array}{c}\text { Non- } \\
\text { significant }\end{array}$ & & Significant \\
\hline $\begin{array}{l}\text { Univariate } \\
\text { analysis }(n=4)\end{array}$ & 30 & & & 26 & $\underline{76,77}$ & & \\
\hline No. of clusters & 1 & 0 & 0 & 1 & 1 & 0 & 0 \\
\hline No. of studies & 1 & 0 & 0 & 1 & 2 & 0 & 0 \\
\hline No. of patients & 160 & 0 & 0 & 59 & 542 & 0 & 0 \\
\hline $\begin{array}{l}\text { Multivariable } \\
\text { analysis }(n=1)\end{array}$ & 30 & & & & & & \\
\hline No. of clusters & 1 & 0 & 0 & 0 & 0 & 0 & 0 \\
\hline No. of studies & 1 & 0 & 0 & 0 & 0 & 0 & 0 \\
\hline No. of patients & 160 & 0 & 0 & 0 & 0 & 0 & 0 \\
\hline
\end{tabular}

\begin{tabular}{|c|c|c|c|c|c|c|c|}
\hline \multirow{2}{*}{$\begin{array}{l}\text { Ocular perfusion } \\
\text { pressure (OAG) }\end{array}$} & \multicolumn{3}{|c|}{ More progression } & \multirow[b]{2}{*}{ No relation } & \multicolumn{3}{|c|}{ Less progression } \\
\hline & Significant & & $\begin{array}{c}\text { Non- } \\
\text { significant }\end{array}$ & & $\begin{array}{c}\text { Non- } \\
\text { significant }\end{array}$ & & Significant \\
\hline $\begin{array}{l}\text { Univariate } \\
\text { analysis }(n=3)\end{array}$ & & & & & $\underline{72,73}$ & 61 & \\
\hline No. of clusters & 0 & 0 & 0 & 0 & 1 & 1 & 0 \\
\hline No. of studies & 0 & 0 & 0 & 0 & 2 & 1 & 0 \\
\hline No. of patients & 0 & 0 & 0 & 0 & 186 & 255 & 0 \\
\hline $\begin{array}{l}\text { Multivariable } \\
\text { analysis }(n=3)\end{array}$ & & & & 62 & 73 & & 61 \\
\hline No. of clusters & 0 & 0 & 0 & 1 & 1 & 0 & 1 \\
\hline No. of studies & 0 & 0 & 0 & 1 & 1 & 0 & 1 \\
\hline No. of patients & 0 & 0 & 0 & 255 & 146 & 0 & 255 \\
\hline
\end{tabular}




\begin{tabular}{|c|c|c|c|c|c|c|c|}
\hline \multirow[b]{2}{*}{ Pulse rate (OAG) } & \multicolumn{3}{|c|}{ More progression } & \multirow[b]{2}{*}{ No relation } & \multicolumn{3}{|c|}{ Less progression } \\
\hline & Significant & & $\begin{array}{c}\text { Non- } \\
\text { significant }\end{array}$ & & $\begin{array}{c}\text { Non- } \\
\text { significant }\end{array}$ & & Significant \\
\hline $\begin{array}{l}\text { Univariate } \\
\text { analysis }(n=1)\end{array}$ & & & & 30 & & & \\
\hline No. of clusters & 0 & 0 & 0 & 1 & 0 & 0 & 0 \\
\hline No. of studies & 0 & 0 & 0 & 1 & 0 & 0 & 0 \\
\hline No. of patients & 0 & 0 & 0 & 160 & 0 & 0 & 0 \\
\hline $\begin{array}{l}\text { Multivariable } \\
\text { analysis }(n=2)\end{array}$ & 24 & & & 22 & & & \\
\hline No. of clusters & 1 & 0 & 0 & 1 & 0 & 0 & 0 \\
\hline No. of studies & 1 & 0 & 0 & 1 & 0 & 0 & 0 \\
\hline No. of patients & 70 & 0 & 0 & 110 & 0 & 0 & 0 \\
\hline
\end{tabular}

\begin{tabular}{|c|c|c|c|c|c|c|c|}
\hline \multirow{2}{*}{$\begin{array}{l}\text { Thinner central } \\
\text { corneal thickness } \\
\text { (NTG) }\end{array}$} & \multicolumn{3}{|c|}{ More progression } & \multirow[b]{2}{*}{ No relation } & \multicolumn{3}{|c|}{ Less progression } \\
\hline & Significant & & $\begin{array}{c}\text { Non- } \\
\text { significant }\end{array}$ & & $\begin{array}{c}\text { Non- } \\
\text { significant }\end{array}$ & & Significant \\
\hline $\begin{array}{l}\text { Univariate } \\
\text { analysis }(n=3)\end{array}$ & $\underline{76,77}$ & & & & 74 & & \\
\hline No. of clusters & 1 & 0 & 0 & 0 & 1 & 0 & 0 \\
\hline No. of studies & 2 & 0 & 0 & 0 & 1 & 0 & 0 \\
\hline No. of patients & 542 & 0 & 0 & 0 & 101 & 0 & 0 \\
\hline $\begin{array}{l}\text { Multivariable } \\
\text { analysis }(n=2)\end{array}$ & 76 & & & 77 & & & \\
\hline No. of clusters & 1 & 0 & 0 & 1 & 0 & 0 & 0 \\
\hline No. of studies & 1 & 0 & 0 & 1 & 0 & 0 & 0 \\
\hline No. of patients & 286 & 0 & 0 & 256 & 0 & 0 & 0 \\
\hline
\end{tabular}

\begin{tabular}{|c|c|c|c|c|c|c|c|}
\hline \multirow{2}{*}{$\begin{array}{l}\text { Infracentral } \\
\text { scotoma localiza- } \\
\text { tion (OAG) }\end{array}$} & \multicolumn{3}{|c|}{ More progression } & \multirow[b]{2}{*}{ No relation } & \multicolumn{3}{|c|}{ Less progression } \\
\hline & Significant & & $\begin{array}{c}\text { Non- } \\
\text { significant }\end{array}$ & & $\begin{array}{c}\text { Non- } \\
\text { significant }\end{array}$ & & Significant \\
\hline $\begin{array}{l}\text { Univariate } \\
\text { analysis }(n=3)\end{array}$ & 8 & 7 & & 4 & & & \\
\hline No. of clusters & 1 & 1 & 0 & 1 & 0 & 0 & 0 \\
\hline No. of studies & 1 & 1 & 0 & 1 & 0 & 0 & 0 \\
\hline No. of patients & 153 & 197 & 0 & 26 & 0 & 0 & 0 \\
\hline \multicolumn{8}{|l|}{$\begin{array}{l}\text { Multivariable } \\
\text { analysis }(n=0)\end{array}$} \\
\hline No. of clusters & 0 & 0 & 0 & 0 & 0 & 0 & 0 \\
\hline No. of studies & 0 & 0 & 0 & 0 & 0 & 0 & 0 \\
\hline No. of patients & 0 & 0 & 0 & 0 & 0 & 0 & 0 \\
\hline
\end{tabular}




\begin{tabular}{|c|c|c|c|c|c|c|c|}
\hline \multirow{2}{*}{$\begin{array}{l}\text { Peripapillary } \\
\text { atrophy (OAG) }\end{array}$} & \multicolumn{3}{|c|}{ More progression } & \multirow[b]{2}{*}{ No relation } & \multicolumn{3}{|c|}{ Less progression } \\
\hline & Significant & & $\begin{array}{c}\text { Non- } \\
\text { significant }\end{array}$ & & $\begin{array}{c}\text { Non- } \\
\text { significant }\end{array}$ & & Significant \\
\hline $\begin{array}{l}\text { Univariate } \\
\text { analysis }(n=2)\end{array}$ & 82 & & & 65 & & & \\
\hline No. of clusters & 1 & 0 & 0 & 1 & 0 & 0 & 0 \\
\hline No. of studies & 1 & 0 & 0 & 1 & 0 & 0 & 0 \\
\hline No. of patients & 245 & 0 & 0 & 108 & 0 & 0 & 0 \\
\hline $\begin{array}{l}\text { Multivariable } \\
\text { analysis }(n=2)\end{array}$ & & 82 & & 21 & & & \\
\hline No. of clusters & 0 & 1 & 0 & 1 & 0 & 0 & 0 \\
\hline No. of studies & 0 & 1 & 0 & 1 & 0 & 0 & 0 \\
\hline No. of patients & 0 & 245 & 0 & 32 & 0 & 0 & 0 \\
\hline
\end{tabular}

\begin{tabular}{|c|c|c|c|c|c|}
\hline \multirow{2}{*}{$\begin{array}{l}\text { Normal-tension } \\
\text { glaucoma (OAG) }\end{array}$} & \multicolumn{2}{|c|}{ More progression } & \multirow[b]{2}{*}{ No relation } & \multicolumn{2}{|c|}{ Less progression } \\
\hline & Significant & $\begin{array}{c}\text { Non- } \\
\text { significant }\end{array}$ & & $\begin{array}{c}\text { Non- } \\
\text { significant }\end{array}$ & Significant \\
\hline
\end{tabular}

\begin{tabular}{|c|c|c|c|c|c|c|c|}
\hline $\begin{array}{l}\text { Univariate } \\
\text { analysis }(n=3)\end{array}$ & & & 18,29 & & & & 63 \\
\hline No. of clusters & 0 & 0 & 2 & 0 & 0 & 0 & 1 \\
\hline No. of studies & 0 & 0 & 2 & 0 & 0 & 0 & 1 \\
\hline No. of patients & 0 & 0 & 185 & 0 & 0 & 0 & 118 \\
\hline \multicolumn{8}{|l|}{$\begin{array}{l}\text { Multivariable } \\
\text { analysis }(n=0)\end{array}$} \\
\hline No. of clusters & 0 & 0 & 0 & 0 & 0 & 0 & 0 \\
\hline No. of studies & 0 & 0 & 0 & 0 & 0 & 0 & 0 \\
\hline No. of patients & 0 & 0 & 0 & 0 & 0 & 0 & 0 \\
\hline
\end{tabular}

\begin{tabular}{|c|c|c|c|c|c|c|c|}
\hline \multirow{2}{*}{$\begin{array}{l}\text { Ocular blood flow } \\
\text { end diastolic } \\
\text { velocity (OAG) }\end{array}$} & \multicolumn{3}{|c|}{ More progression } & \multirow[b]{2}{*}{ No relation } & \multicolumn{3}{|c|}{ Less progression } \\
\hline & Significant & & $\begin{array}{c}\text { Non- } \\
\text { significant }\end{array}$ & & $\begin{array}{c}\text { Non- } \\
\text { significant }\end{array}$ & & Significant \\
\hline $\begin{array}{l}\text { Univariate } \\
\text { analysis }(n=2)\end{array}$ & & $72^{+}$ & & $72^{\S}$ & $73^{+}$ & & $72^{\ddagger}, 73^{\ddagger}, 73^{5}$ \\
\hline No. of clusters & 0 & 1 & 0 & 1 & 1 & 0 & 1 \\
\hline No. of studies & 0 & 1 & 0 & 1 & 1 & 0 & 2 \\
\hline No. of patients & 0 & 40 & 0 & 40 & 146 & 0 & 186 \\
\hline $\begin{array}{l}\text { Multivariable } \\
\text { analysis }(n=2)\end{array}$ & & & & $\underline{72^{+}, 72^{8}}$ & & & $\underline{72^{\ddagger}, 73^{\ddagger}, 73^{\S}}$ \\
\hline No. of clusters & 0 & 0 & 0 & 1 & 0 & 0 & 1 \\
\hline No. of studies & 0 & 0 & 0 & 1 & 0 & 0 & 2 \\
\hline No. of patients & 0 & 0 & 0 & 40 & 0 & 0 & 186 \\
\hline
\end{tabular}

†In central retinal artery; ¥In ophthalmic artery; §In short posterior ciliary arteries. 


\begin{tabular}{|c|c|c|c|c|c|c|c|}
\hline \multirow{2}{*}{$\begin{array}{l}\text { Systolic ocular } \\
\text { perfusion pressure } \\
\text { (OAG) }\end{array}$} & \multicolumn{3}{|c|}{ More progression } & \multirow[b]{2}{*}{ No relation } & \multicolumn{3}{|c|}{ Less progression } \\
\hline & Significant & & $\begin{array}{c}\text { Non- } \\
\text { significant }\end{array}$ & & $\begin{array}{c}\text { Non- } \\
\text { significant }\end{array}$ & & Significant \\
\hline $\begin{array}{l}\text { Univariate } \\
\text { analysis }(n=1)\end{array}$ & & & & & & & 61 \\
\hline No. of clusters & 0 & 0 & 0 & 0 & 0 & 0 & 1 \\
\hline No. of studies & 0 & 0 & 0 & 0 & 0 & 0 & 1 \\
\hline No. of patients & 0 & 0 & 0 & 0 & 0 & 0 & 255 \\
\hline $\begin{array}{l}\text { Multivariable } \\
\text { analysis }(n=2)\end{array}$ & & & & 62 & & & 61 \\
\hline No. of clusters & 0 & 0 & 0 & 1 & 0 & 0 & 1 \\
\hline No. of studies & 0 & 0 & 0 & 1 & 0 & 0 & 1 \\
\hline No. of patients & 0 & 0 & 0 & 255 & 0 & 0 & 255 \\
\hline
\end{tabular}

\begin{tabular}{|c|c|c|c|c|c|}
\hline \multirow{2}{*}{$\begin{array}{l}\text { Family history of } \\
\text { diabetes (OAG) }\end{array}$} & \multicolumn{2}{|c|}{ More progression } & \multirow[b]{2}{*}{ No relation } & \multicolumn{2}{|c|}{ Less progression } \\
\hline & Significant & $\begin{array}{c}\text { Non- } \\
\text { significant }\end{array}$ & & $\begin{array}{c}\text { Non- } \\
\text { significant }\end{array}$ & Significant \\
\hline
\end{tabular}

Univariate

analysis $(n=1)$

\begin{tabular}{|c|c|c|c|c|c|c|}
\hline No. of clusters & 0 & 0 & 0 & 0 & 0 & 0 \\
\hline No. of studies & 0 & 0 & 0 & 0 & 0 & 0 \\
\hline No. of patients & 0 & 0 & 0 & 0 & 0 & 0 \\
\hline
\end{tabular}

Multivariable

analysis $(n=0)$

\begin{tabular}{llllllll} 
No. of clusters & 0 & 0 & 0 & 0 & 0 & 0 & 0 \\
No. of studies & 0 & 0 & 0 & 0 & 0 & 0 & 0 \\
No. of patients & 0 & 0 & 0 & 0 & 0 & 0 & 0 \\
\hline
\end{tabular}

\begin{tabular}{|c|c|c|c|c|c|c|c|}
\hline \multirow{2}{*}{$\begin{array}{l}\text { Variant of the } \\
\text { myocilin } \\
\text { (mt. } 1(+))(O A G)\end{array}$} & \multicolumn{3}{|c|}{ More progression } & \multirow[b]{2}{*}{ No relation } & \multicolumn{3}{|c|}{ Less progression } \\
\hline & Significant & & $\begin{array}{c}\text { Non- } \\
\text { significant }\end{array}$ & & $\begin{array}{c}\text { Non- } \\
\text { significant }\end{array}$ & & Significant \\
\hline \multicolumn{8}{|l|}{$\begin{array}{l}\text { Univariate } \\
\text { analysis }(n=0)\end{array}$} \\
\hline No. of clusters & 0 & 0 & 0 & 0 & 0 & 0 & 0 \\
\hline No. of studies & 0 & 0 & 0 & 0 & 0 & 0 & 0 \\
\hline No. of patients & 0 & 0 & 0 & 0 & 0 & 0 & 0 \\
\hline $\begin{array}{l}\text { Multivariable } \\
\text { analysis }(n=1)\end{array}$ & 57 & & & & & & \\
\hline No. of clusters & 1 & 0 & 0 & 0 & 0 & 0 & 0 \\
\hline No. of studies & 1 & 0 & 0 & 0 & 0 & 0 & 0 \\
\hline No. of patients & 135 & 0 & 0 & 0 & 0 & 0 & 0 \\
\hline
\end{tabular}




\begin{tabular}{|c|c|c|c|c|c|c|c|}
\hline \multirow{2}{*}{$\begin{array}{l}\text { Asian descent } \\
(\mathrm{OAG})\end{array}$} & \multicolumn{3}{|c|}{ More progression } & \multirow[b]{2}{*}{ No relation } & \multicolumn{3}{|c|}{ Less progression } \\
\hline & Significant & & $\begin{array}{c}\text { Non- } \\
\text { significant }\end{array}$ & & $\begin{array}{c}\text { Non- } \\
\text { significant }\end{array}$ & & Significant \\
\hline $\begin{array}{l}\text { Univariate } \\
\text { analysis }(n=1)\end{array}$ & & & & & & & 30 \\
\hline No. of clusters & 0 & 0 & 0 & 0 & 0 & 0 & 1 \\
\hline No. of studies & 0 & 0 & 0 & 0 & 0 & 0 & 1 \\
\hline No. of patients & 0 & 0 & 0 & 0 & 0 & 0 & 160 \\
\hline $\begin{array}{l}\text { Multivariable } \\
\text { analysis }(n=0)\end{array}$ & & & & & & & \\
\hline No. of clusters & 0 & 0 & 0 & 0 & 0 & 0 & 0 \\
\hline No. of studies & 0 & 0 & 0 & 0 & 0 & 0 & 0 \\
\hline No. of patients & 0 & 0 & 0 & 0 & 0 & 0 & 0 \\
\hline
\end{tabular}

\begin{tabular}{|c|c|c|c|c|c|c|c|}
\hline \multirow{2}{*}{$\begin{array}{l}\text { Red blood cell } \\
\text { distribution width } \\
\text { (OAG) }\end{array}$} & \multicolumn{3}{|c|}{ More progression } & \multirow[b]{2}{*}{ No relation } & \multicolumn{3}{|c|}{ Less progression } \\
\hline & Significant & & $\begin{array}{c}\text { Non- } \\
\text { significant }\end{array}$ & & $\begin{array}{c}\text { Non- } \\
\text { significant }\end{array}$ & & Significant \\
\hline $\begin{array}{l}\text { Univariate } \\
\text { analysis }(n=1)\end{array}$ & & & & & & & 70 \\
\hline No. of clusters & 0 & 0 & 0 & 0 & 0 & 0 & 1 \\
\hline No. of studies & 0 & 0 & 0 & 0 & 0 & 0 & 1 \\
\hline No. of patients & 0 & 0 & 0 & 0 & 0 & 0 & 258 \\
\hline \multicolumn{8}{|l|}{$\begin{array}{l}\text { Multivariable } \\
\text { analysis }(n=0)\end{array}$} \\
\hline No. of clusters & 0 & 0 & 0 & 0 & 0 & 0 & 0 \\
\hline No. of studies & 0 & 0 & 0 & 0 & 0 & 0 & 0 \\
\hline No. of patients & 0 & 0 & 0 & 0 & 0 & 0 & 0 \\
\hline
\end{tabular}

\begin{tabular}{|c|c|c|c|c|c|c|c|}
\hline \multirow{2}{*}{$\begin{array}{l}\text { Threat to fixation } \\
\text { (NTG) }\end{array}$} & \multicolumn{3}{|c|}{ More progression } & \multirow[b]{2}{*}{ No relation } & \multicolumn{3}{|c|}{ Less progression } \\
\hline & Significant & & $\begin{array}{c}\text { Non- } \\
\text { significant }\end{array}$ & & $\begin{array}{c}\text { Non- } \\
\text { significant }\end{array}$ & & Significant \\
\hline $\begin{array}{l}\text { Univariate } \\
\text { analysis }(n=1)\end{array}$ & & 31 & & & & & \\
\hline No. of clusters & 0 & 1 & 0 & 0 & 0 & 0 & 0 \\
\hline No. of studies & 0 & 1 & 0 & 0 & 0 & 0 & 0 \\
\hline No. of patients & 0 & 152 & 0 & 0 & 0 & 0 & 0 \\
\hline $\begin{array}{l}\text { Multivariable } \\
\text { analysis }(n=1)\end{array}$ & & 31 & & & & & \\
\hline No. of clusters & 0 & 1 & 0 & 0 & 0 & 0 & 0 \\
\hline No. of studies & 0 & 1 & 0 & 0 & 0 & 0 & 0 \\
\hline No. of patients & 0 & 152 & 0 & 0 & 0 & 0 & 0 \\
\hline
\end{tabular}




\begin{tabular}{|c|c|c|c|c|c|c|c|}
\hline \multirow{2}{*}{$\begin{array}{l}\text { Outflow facility/ } \\
\text { water drinking test/ } \\
\text { diurnal IOP } \\
\text { variation (OAG) }\end{array}$} & \multicolumn{3}{|c|}{ More progression } & \multirow[b]{2}{*}{ No relation } & \multicolumn{3}{|c|}{ Less progression } \\
\hline & Significant & & $\begin{array}{c}\text { Non- } \\
\text { significant }\end{array}$ & & $\begin{array}{l}\text { Non- } \\
\text { significant }\end{array}$ & & Significant \\
\hline $\begin{array}{l}\text { Univariate } \\
\text { analysis }(n=1)\end{array}$ & 14 & & & & & & \\
\hline No. of clusters & 1 & 0 & 0 & 0 & 0 & 0 & 0 \\
\hline No. of studies & 1 & 0 & 0 & 0 & 0 & 0 & 0 \\
\hline No. of patients & 54 & 0 & 0 & 0 & 0 & 0 & 0 \\
\hline \multicolumn{8}{|l|}{$\begin{array}{l}\text { Multivariable } \\
\text { analysis }(n=0)\end{array}$} \\
\hline No. of clusters & 0 & 0 & 0 & 0 & 0 & 0 & 0 \\
\hline No. of studies & 0 & 0 & 0 & 0 & 0 & 0 & 0 \\
\hline No. of patients & 0 & 0 & 0 & 0 & 0 & 0 & 0 \\
\hline
\end{tabular}

\begin{tabular}{|c|c|c|c|c|c|}
\hline \multirow{2}{*}{$\begin{array}{l}\text { IOP increase after } \\
\text { pupillary dilatation } \\
\text { (OAG) }\end{array}$} & \multicolumn{2}{|c|}{ More progression } & \multirow[b]{2}{*}{ No relation } & \multicolumn{2}{|c|}{ Less progression } \\
\hline & Significant & $\begin{array}{c}\text { Non- } \\
\text { significant }\end{array}$ & & $\begin{array}{c}\text { Non- } \\
\text { significant }\end{array}$ & Significant \\
\hline
\end{tabular}

\section{Univariate}

analysis $(n=1)$

No. of studies

No. of patients

Multivariable

analysis $(n=0)$

\begin{tabular}{llllllll} 
No. of clusters & 0 & 0 & 0 & 0 & 0 & 0 & 0 \\
No. of studies & 0 & 0 & 0 & 0 & 0 & 0 & 0 \\
No. of patients & 0 & 0 & 0 & 0 & 0 & 0 & 0 \\
\hline
\end{tabular}

\begin{tabular}{|c|c|c|c|c|c|}
\hline \multirow{2}{*}{$\begin{array}{l}\text { Highest IOP ever } \\
\text { recorded before } \\
\text { baseline (OAG) }\end{array}$} & \multicolumn{2}{|c|}{ More progression } & \multirow[b]{2}{*}{ No relation } & \multicolumn{2}{|c|}{ Less progression } \\
\hline & Significant & $\begin{array}{c}\text { Non- } \\
\text { significant }\end{array}$ & & $\begin{array}{c}\text { Non- } \\
\text { significant }\end{array}$ & Significant \\
\hline
\end{tabular}

Univariate

analysis $(n=1)$

$\begin{array}{lccccccc}\text { No. of clusters } & 1 & 0 & 0 & 0 & 0 & 0 & 0 \\ \text { No. of studies } & 1 & 0 & 0 & 0 & 0 & 0 & 0 \\ \text { No. of patients } & 70 & 0 & 0 & 0 & 0 & 0 & 0\end{array}$

Multivariable

analysis ( $n=0)$

\begin{tabular}{llllllll} 
No. of clusters & 0 & 0 & 0 & 0 & 0 & 0 & 0 \\
No. of studies & 0 & 0 & 0 & 0 & 0 & 0 & 0 \\
No. of patients & 0 & 0 & 0 & 0 & 0 & 0 & 0 \\
\hline
\end{tabular}


Chapter 2

\begin{tabular}{|c|c|c|c|c|c|c|c|}
\hline \multirow{2}{*}{$\begin{array}{l}\text { Ocular perfusion } \\
\text { pressure fluctua- } \\
\text { tion (OAG) }\end{array}$} & \multicolumn{3}{|c|}{ More progression } & \multirow[b]{2}{*}{ No relation } & \multicolumn{3}{|c|}{ Less progression } \\
\hline & Significant & & $\begin{array}{c}\text { Non- } \\
\text { significant }\end{array}$ & & $\begin{array}{c}\text { Non- } \\
\text { significant }\end{array}$ & & Significant \\
\hline $\begin{array}{l}\text { Univariate } \\
\text { analysis }(n=1)\end{array}$ & & 74 & & & & & \\
\hline No. of clusters & 0 & 1 & 0 & 0 & 0 & 0 & 0 \\
\hline No. of studies & 0 & 1 & 0 & 0 & 0 & 0 & 0 \\
\hline No. of patients & 0 & 101 & 0 & 0 & 0 & 0 & 0 \\
\hline $\begin{array}{l}\text { Multivariable } \\
\text { analysis }(n=1)\end{array}$ & & 74 & & & & & \\
\hline No. of clusters & 0 & 1 & 0 & 0 & 0 & 0 & 0 \\
\hline No. of studies & 0 & 1 & 0 & 0 & 0 & 0 & 0 \\
\hline No. of patients & 0 & 101 & 0 & 0 & 0 & 0 & 0 \\
\hline
\end{tabular}


Evidence tables of prognostic factors without evidence of an association with glaucomatous visual field progression

\begin{tabular}{|c|c|c|c|c|c|c|c|}
\hline \multirow{2}{*}{$\begin{array}{l}\text { Family history of } \\
\text { glaucoma (OAG) }\end{array}$} & \multicolumn{3}{|c|}{ More progression } & \multirow[b]{2}{*}{ No relation } & \multicolumn{3}{|c|}{ Less progression } \\
\hline & Significant & & $\begin{array}{c}\text { Non- } \\
\text { significant }\end{array}$ & & $\begin{array}{c}\text { Non- } \\
\text { significant }\end{array}$ & & Significant \\
\hline $\begin{array}{l}\text { Univariate } \\
\text { analysis }(n=12)\end{array}$ & & 2 & $\begin{array}{c}12,50 \\
70\end{array}$ & $\begin{array}{c}9,13,30 *, 48 \\
51,61,65\end{array}$ & 60 & & \\
\hline No. of clusters & 0 & 1 & 3 & 7 & 1 & 0 & 0 \\
\hline No. of studies & 0 & 1 & 3 & 7 & 1 & 0 & 0 \\
\hline No. of patients & 0 & 57 & 386 & 1027 & 255 & 0 & 0 \\
\hline $\begin{array}{l}\text { Multivariable } \\
\text { analysis }(n=7)\end{array}$ & 13 & & 50,57 & $16^{*}, 55, \underline{60,62}$ & & & \\
\hline No. of clusters & 1 & 0 & 2 & 3 & 0 & 0 & 0 \\
\hline No. of studies & 1 & 0 & 2 & 4 & 0 & 0 & 0 \\
\hline No. of patients & 57 & 0 & 223 & 1173 & 0 & 0 & 0 \\
\hline \multirow{2}{*}{$\begin{array}{l}\text { Atherosclero- } \\
\text { sis (OAG) }\end{array}$} & \multicolumn{3}{|c|}{ More progression } & & \multicolumn{3}{|c|}{ Less progression } \\
\hline & Significant & & $\begin{array}{c}\text { Non- } \\
\text { significant }\end{array}$ & No relation & $\begin{array}{c}\text { Non- } \\
\text { significant }\end{array}$ & & Significant \\
\hline $\begin{array}{l}\text { Univariate } \\
\text { analysis }(n=12)\end{array}$ & & & $\begin{array}{c}67,70, \\
\underline{76 *, 77^{*}}, 80\end{array}$ & $\frac{4,26 *, 27}{30 *, 60}$ & 41,61 & & \\
\hline No. of clusters & 0 & 0 & 4 & 4 & 2 & 0 & 0 \\
\hline No. of studies & 0 & 0 & 5 & 5 & 2 & 0 & 0 \\
\hline No. of patients & 0 & 0 & 1151 & 570 & 656 & 0 & 0 \\
\hline $\begin{array}{l}\text { Multivariable } \\
\text { analysis }(n=4)\end{array}$ & & & & $39, \underline{60,62}$ & 67 & & \\
\hline No. of clusters & 0 & 0 & 0 & 2 & 1 & 0 & 0 \\
\hline No. of studies & 0 & 0 & 0 & 3 & 1 & 0 & 0 \\
\hline No. of patients & 0 & 0 & 0 & 1068 & 58 & 0 & 0 \\
\hline \multirow{2}{*}{$\begin{array}{l}\text { Systemic hyper- } \\
\text { tension (OAG) }\end{array}$} & \multicolumn{3}{|c|}{ More progression } & & \multicolumn{3}{|c|}{ Less progression } \\
\hline & Significant & & $\begin{array}{c}\text { Non- } \\
\text { significant }\end{array}$ & No relation & $\begin{array}{c}\text { Non- } \\
\text { significant }\end{array}$ & & Significant \\
\hline $\begin{array}{l}\text { Univariate } \\
\text { analysis }(n=11)\end{array}$ & 41 & & 38,70 & $9,27,61,65$ & $12,18,42,60$ & & \\
\hline No. of clusters & 1 & 0 & 2 & 4 & 4 & 0 & 0 \\
\hline No. of studies & 1 & 0 & 2 & 4 & 4 & 0 & 0 \\
\hline No. of patients & 401 & 0 & 559 & 715 & 422 & 0 & 0 \\
\hline $\begin{array}{l}\text { Multivariable } \\
\text { analysis }(n=6)\end{array}$ & & & $\underline{38,41}$ & $39,55, \underline{60,62}$ & & & \\
\hline No. of clusters & 0 & 0 & 1 & 3 & 0 & 0 & 0 \\
\hline No. of studies & 0 & 0 & 2 & 4 & 0 & 0 & 0 \\
\hline No. of patients & 0 & 0 & 702 & 1675 & 0 & 0 & 0 \\
\hline
\end{tabular}

*Studies comprising a majority of normal-tension glaucoma patients. 


\begin{tabular}{|c|c|c|c|c|c|c|c|}
\hline \multirow{2}{*}{$\begin{array}{l}\text { Visual acuity } \\
\text { (OAG) }\end{array}$} & \multicolumn{3}{|c|}{ More progression } & \multirow[b]{2}{*}{ No relation } & \multicolumn{3}{|c|}{ Less progression } \\
\hline & Significant & & $\begin{array}{c}\text { Non- } \\
\text { significant }\end{array}$ & & $\begin{array}{c}\text { Non- } \\
\text { significant }\end{array}$ & & Significant \\
\hline $\begin{array}{l}\text { Univariate } \\
\text { analysis }(n=8)\end{array}$ & & & 41 & $30 *, 51,71$ & $\begin{array}{r}12,44 \\
76^{*}, 77^{*} \\
\end{array}$ & & \\
\hline No. of clusters & 0 & 0 & 1 & 3 & 3 & 0 & 0 \\
\hline No. of studies & 0 & 0 & 1 & 3 & 4 & 0 & 0 \\
\hline No. of patients & 0 & 0 & 401 & 596 & 695 & 0 & 0 \\
\hline $\begin{array}{l}\text { Multivariable } \\
\text { analysis ( } n=7)\end{array}$ & & & $\underline{38,41}$ & $\begin{array}{c}17 \\
22^{*}, 24^{*}, 25^{*}\end{array}$ & & 39 & \\
\hline No. of clusters & 0 & 0 & 1 & 2 & 0 & 1 & 0 \\
\hline No. of studies & 0 & 0 & 2 & 4 & 0 & 1 & 0 \\
\hline No. of patients & 0 & 0 & 702 & 376 & 0 & 558 & 0 \\
\hline
\end{tabular}

\begin{tabular}{|c|c|c|c|c|c|c|c|}
\hline \multirow[b]{2}{*}{ Female sex (NTG) } & \multicolumn{3}{|c|}{ More progression } & \multirow[b]{2}{*}{ No relation } & \multicolumn{3}{|c|}{ Less progression } \\
\hline & Significant & & $\begin{array}{c}\text { Non- } \\
\text { significant }\end{array}$ & & $\begin{array}{c}\text { Non- } \\
\text { significant }\end{array}$ & & Significant \\
\hline $\begin{array}{l}\text { Univariate } \\
\text { analysis }(n=9)\end{array}$ & 30 & & $\begin{array}{c}32, \underline{35,34} \\
52,74, \underline{76,77}\end{array}$ & 10 & & & \\
\hline No. of clusters & 1 & 0 & 5 & 1 & 0 & 0 & 0 \\
\hline No. of studies & 1 & 0 & 7 & 1 & 0 & 0 & 0 \\
\hline No. of patients & 160 & 0 & 859 & 36 & 0 & 0 & 0 \\
\hline
\end{tabular}

Multivariable analysis $(n=6)$

$30 \quad 16, \frac{22,24,25}{34}$

\begin{tabular}{|c|c|c|c|c|c|c|c|}
\hline No. of clusters & 0 & 0 & 1 & 3 & 0 & 0 & 0 \\
\hline No. of studies & 0 & 0 & 1 & 5 & 0 & 0 & 0 \\
\hline No. of patients & 0 & 0 & 160 & 300 & 0 & 0 & 0 \\
\hline \multirow{2}{*}{$\begin{array}{l}\text { Systolic blood } \\
\text { pressure (OAG) }\end{array}$} & \multicolumn{3}{|c|}{ More progression } & \multirow[b]{2}{*}{ No relation } & \multicolumn{3}{|c|}{ Less progression } \\
\hline & Significant & & $\begin{array}{c}\text { Non- } \\
\text { significant }\end{array}$ & & $\begin{array}{c}\text { Non- } \\
\text { significant }\end{array}$ & & Significant \\
\hline $\begin{array}{l}\text { Univariate } \\
\text { analysis }(n=8)\end{array}$ & & 2 & $73,74^{*}$ & $30 *, 70$ & 60,64 & & 61 \\
\hline No. of clusters & 0 & 1 & 2 & 2 & 2 & 0 & 1 \\
\hline No. of studies & 0 & 1 & 2 & 2 & 2 & 0 & 1 \\
\hline No. of patients & 0 & 57 & 247 & 418 & 304 & 0 & 255 \\
\hline $\begin{array}{l}\text { Multivariable } \\
\text { analysis }(n=10)\end{array}$ & & $\frac{22^{*}}{\underline{24^{*}}}$ & & $\underline{60,62}, 74^{*}$ & $2,23^{*}, 61,64$ & & $25^{*}$ \\
\hline No. of clusters & 0 & 1 & 0 & 2 & 4 & 0 & 1 \\
\hline No. of studies & 0 & 2 & 0 & 3 & 4 & 0 & 1 \\
\hline No. of patients & 0 & 180 & 0 & 611 & 408 & 0 & 28 \\
\hline
\end{tabular}

*Studies comprising a majority of normal-tension glaucoma patients. 


\begin{tabular}{|c|c|c|c|c|c|c|c|}
\hline \multirow{2}{*}{$\begin{array}{l}\text { Myopic refractive } \\
\text { error (NTG) }\end{array}$} & \multicolumn{3}{|c|}{ More progression } & \multirow[b]{2}{*}{ No relation } & \multicolumn{3}{|c|}{ Less progression } \\
\hline & Significant & & $\begin{array}{c}\text { Non- } \\
\text { significant }\end{array}$ & & $\begin{array}{l}\text { Non- } \\
\text { significant }\end{array}$ & & Significant \\
\hline $\begin{array}{l}\text { Univariate } \\
\text { analysis }(n=6)\end{array}$ & & & $\underline{34,35}$ & $26,30,83$ & 74 & & \\
\hline No. of clusters & 0 & 0 & 1 & 3 & 1 & 0 & 0 \\
\hline No. of studies & 0 & 0 & 2 & 3 & 1 & 0 & 0 \\
\hline No. of patients & 0 & 0 & 128 & 362 & 101 & 0 & 0 \\
\hline $\begin{array}{l}\text { Multivariable } \\
\text { analysis }(n=8)\end{array}$ & & & 21,83 & $16, \frac{22,24,25}{\underline{34,35}}$ & & & \\
\hline No. of clusters & 0 & 0 & 2 & 3 & 0 & 0 & 0 \\
\hline No. of studies & 0 & 0 & 2 & 6 & 0 & 0 & 0 \\
\hline No. of patients & 0 & 0 & 175 & 392 & 0 & 0 & 0 \\
\hline
\end{tabular}

\begin{tabular}{|c|c|c|c|c|c|c|c|}
\hline \multirow{2}{*}{$\begin{array}{l}\text { Raynaud's } \\
\text { phenomenon } \\
\text { (OAG) }\end{array}$} & \multicolumn{3}{|c|}{ More progression } & \multirow[b]{2}{*}{ No relation } & \multicolumn{3}{|c|}{ Less progression } \\
\hline & Significant & & $\begin{array}{c}\text { Non- } \\
\text { significant }\end{array}$ & & $\begin{array}{c}\text { Non- } \\
\text { significant }\end{array}$ & & Significant \\
\hline $\begin{array}{l}\text { Univariate } \\
\text { analysis }(n=9)\end{array}$ & & & $18,30^{*}, 60$ & 61,65 & $\frac{76^{*}, 77^{*}}{\underline{80,81}}$ & & \\
\hline No. of clusters & 0 & 0 & 3 & 2 & 2 & 0 & 0 \\
\hline No. of studies & 0 & 0 & 3 & 2 & 4 & 0 & 0 \\
\hline No. of patients & 0 & 0 & 498 & 363 & 911 & 0 & 0 \\
\hline
\end{tabular}

Multivariable analysis $(n=2)$

$$
\underline{60,62}
$$

\begin{tabular}{|c|c|c|c|c|c|c|c|}
\hline No. of clusters & 0 & 0 & 0 & 1 & 0 & 0 & 0 \\
\hline No. of studies & 0 & 0 & 0 & 2 & 0 & 0 & 0 \\
\hline No. of patients & 0 & 0 & 0 & 510 & 0 & 0 & 0 \\
\hline \multirow[b]{2}{*}{ Baseline IOP (NTG) } & \multicolumn{3}{|c|}{ More progression } & \multirow[b]{2}{*}{ No relation } & \multicolumn{3}{|c|}{ Less progression } \\
\hline & Significant & & $\begin{array}{c}\text { Non- } \\
\text { significant }\end{array}$ & & $\begin{array}{c}\text { Non- } \\
\text { significant }\end{array}$ & & Significant \\
\hline $\begin{array}{l}\text { Univariate } \\
\text { analysis }(n=4)\end{array}$ & & & & 10,30 & 35,74 & & \\
\hline No. of clusters & 0 & 0 & 0 & 2 & 2 & 0 & 0 \\
\hline No. of studies & 0 & 0 & 0 & 2 & 2 & 0 & 0 \\
\hline No. of patients & 0 & 0 & 0 & 196 & 193 & 0 & 0 \\
\hline $\begin{array}{l}\text { Multivariable } \\
\text { analysis }(n=7)\end{array}$ & 21 & & & $\frac{22,24,25}{74}, 30$ & & & 35 \\
\hline No. of clusters & 1 & 0 & 0 & 3 & 0 & 0 & 1 \\
\hline No. of studies & 1 & 0 & 0 & 5 & 0 & 0 & 1 \\
\hline No. of patients & 32 & 0 & 0 & 469 & 0 & 0 & 92 \\
\hline
\end{tabular}

*Studies comprising a majority of normal-tension glaucoma patients. 


\begin{tabular}{|c|c|c|c|c|c|c|c|}
\hline \multirow[b]{2}{*}{ Diabetes (NTG) } & \multicolumn{3}{|c|}{ More progression } & \multirow[b]{2}{*}{ No relation } & \multicolumn{3}{|c|}{ Less progression } \\
\hline & Significant & & $\begin{array}{c}\text { Non- } \\
\text { significant }\end{array}$ & & $\begin{array}{c}\text { Non- } \\
\text { significant }\end{array}$ & & Significant \\
\hline $\begin{array}{l}\text { Univariate } \\
\text { analysis }(n=4)\end{array}$ & & & 76 & 26,30 & 77 & & \\
\hline No. of clusters & 0 & 0 & 1 & 2 & 1 & 0 & 0 \\
\hline No. of studies & 0 & 0 & 1 & 2 & 1 & 0 & 0 \\
\hline No. of patients & 0 & 0 & 286 & 219 & 256 & 0 & 0 \\
\hline $\begin{array}{l}\text { Multivariable } \\
\text { analysis }(n=4)\end{array}$ & & & & $16, \underline{21,22,24}$ & & & \\
\hline No. of clusters & 0 & 0 & 0 & 2 & 0 & 0 & 0 \\
\hline No. of studies & 0 & 0 & 0 & 4 & 0 & 0 & 0 \\
\hline No. of patients & 0 & 0 & 0 & 268 & 0 & 0 & 0 \\
\hline
\end{tabular}

\begin{tabular}{|c|c|c|c|c|c|c|c|}
\hline \multirow[b]{2}{*}{ Migraine (OAG) } & \multicolumn{3}{|c|}{ More progression } & \multirow[b]{2}{*}{ No relation } & \multicolumn{3}{|c|}{ Less progression } \\
\hline & Significant & & $\begin{array}{c}\text { Non- } \\
\text { significant }\end{array}$ & & $\begin{array}{c}\text { Non- } \\
\text { significant }\end{array}$ & & Significant \\
\hline $\begin{array}{l}\text { Univariate } \\
\text { analysis }(n=7)\end{array}$ & & & 60 & $27,61,65$ & $70, \underline{80,81}$ & & \\
\hline No. of clusters & 0 & 0 & 1 & 3 & 2 & 0 & 0 \\
\hline No. of studies & 0 & 0 & 1 & 3 & 3 & 0 & 0 \\
\hline No. of patients & 0 & 0 & 255 & 433 & 627 & 0 & 0 \\
\hline $\begin{array}{l}\text { Multivariable } \\
\text { analysis }(n=2)\end{array}$ & & & & $\underline{60,62}$ & & & \\
\hline No. of clusters & 0 & 0 & 0 & 1 & 0 & 0 & 0 \\
\hline No. of studies & 0 & 0 & 0 & 2 & 0 & 0 & 0 \\
\hline No. of patients & 0 & 0 & 0 & 510 & 0 & 0 & 0 \\
\hline
\end{tabular}

\begin{tabular}{|c|c|c|c|c|c|c|c|}
\hline \multirow[b]{2}{*}{ Smoking (OAG) } & \multicolumn{3}{|c|}{ More progression } & \multirow[b]{2}{*}{ No relation } & \multicolumn{3}{|c|}{ Less progression } \\
\hline & Significant & & $\begin{array}{c}\text { Non- } \\
\text { significant }\end{array}$ & & $\begin{array}{c}\text { Non- } \\
\text { significant }\end{array}$ & & Significant \\
\hline $\begin{array}{l}\text { Univariate } \\
\text { analysis }(n=4)\end{array}$ & & & $76^{*}$ & $27, \underline{60,61}$ & & & \\
\hline No. of clusters & 0 & 0 & 1 & 2 & 0 & 0 & 0 \\
\hline No. of studies & 0 & 0 & 1 & 3 & 0 & 0 & 0 \\
\hline No. of patients & 0 & 0 & 286 & 580 & 0 & 0 & 0 \\
\hline $\begin{array}{l}\text { Multivariable } \\
\text { analysis }(n=3)\end{array}$ & & & & $55, \underline{60,62}$ & & & \\
\hline No. of clusters & 0 & 0 & 0 & 2 & 0 & 0 & 0 \\
\hline No. of studies & 0 & 0 & 0 & 3 & 0 & 0 & 0 \\
\hline No. of patients & 0 & 0 & 0 & 1117 & 0 & 0 & 0 \\
\hline
\end{tabular}

*Studies comprising a majority of normal-tension glaucoma patients. 


\begin{tabular}{|c|c|c|c|c|c|c|c|}
\hline \multirow{2}{*}{$\begin{array}{l}\text { Diastolic blood } \\
\text { pressure (NTG) }\end{array}$} & \multicolumn{3}{|c|}{ More progression } & \multirow[b]{2}{*}{ No relation } & \multicolumn{3}{|c|}{ Less progression } \\
\hline & Significant & & $\begin{array}{c}\text { Non- } \\
\text { significant }\end{array}$ & & $\begin{array}{c}\text { Non- } \\
\text { significant }\end{array}$ & & Significant \\
\hline $\begin{array}{l}\text { Univariate } \\
\text { analysis }(n=2)\end{array}$ & & & 74 & 30 & & & \\
\hline No. of clusters & 0 & 0 & 1 & 1 & 0 & 0 & 0 \\
\hline No. of studies & 0 & 0 & 1 & 1 & 0 & 0 & 0 \\
\hline No. of patients & 0 & 0 & 101 & 160 & 0 & 0 & 0 \\
\hline $\begin{array}{l}\text { Multivariable } \\
\text { analysis }(n=4)\end{array}$ & & & 23 & $\underline{22,24,25}$ & & & \\
\hline No. of clusters & 0 & 0 & 1 & 1 & 0 & 0 & 0 \\
\hline No. of studies & 0 & 0 & 1 & 3 & 0 & 0 & 0 \\
\hline No. of patients & 0 & 0 & 47 & 208 & 0 & 0 & 0 \\
\hline
\end{tabular}

\begin{tabular}{|c|c|c|c|c|c|c|c|}
\hline \multirow{2}{*}{$\begin{array}{l}\text { Baseline untreat- } \\
\text { ed IOP (NTG) }\end{array}$} & \multicolumn{3}{|c|}{ More progression } & \multirow[b]{2}{*}{ No relation } & \multicolumn{3}{|c|}{ Less progression } \\
\hline & Significant & & $\begin{array}{c}\text { Non- } \\
\text { significant }\end{array}$ & & $\begin{array}{c}\text { Non- } \\
\text { significant }\end{array}$ & & Significant \\
\hline $\begin{array}{l}\text { Univariate } \\
\text { analysis }(n=3)\end{array}$ & & & & 30 & 35,74 & & \\
\hline No. of clusters & 0 & 0 & 0 & 1 & 2 & 0 & 0 \\
\hline No. of studies & 0 & 0 & 0 & 1 & 2 & 0 & 0 \\
\hline No. of patients & 0 & 0 & 0 & 160 & 193 & 0 & 0 \\
\hline $\begin{array}{l}\text { Multivariable } \\
\text { analysis }(n=6)\end{array}$ & 21 & & & $\underline{22,24}, 30,74$ & & & 35 \\
\hline No. of clusters & 1 & 0 & 0 & 3 & 0 & 0 & 1 \\
\hline No. of studies & 1 & 0 & 0 & 4 & 0 & 0 & 1 \\
\hline No. of patients & 32 & 0 & 0 & 441 & 0 & 0 & 92 \\
\hline
\end{tabular}

\begin{tabular}{|c|c|c|c|c|c|}
\hline \multirow{2}{*}{$\begin{array}{l}\text { Mean diurnal IOP } \\
\text { (OAG) }\end{array}$} & \multicolumn{2}{|c|}{ More progression } & \multirow[b]{2}{*}{ No relation } & \multicolumn{2}{|c|}{ Less progression } \\
\hline & Significant & $\begin{array}{c}\text { Non- } \\
\text { significant }\end{array}$ & & $\begin{array}{c}\text { Non- } \\
\text { significant }\end{array}$ & Significant \\
\hline
\end{tabular}

Univariate

analysis ( $n=2)$

\begin{tabular}{lccccccc} 
No. of clusters & 0 & 0 & 0 & 0 & 2 & 0 & 0 \\
No. of studies & 0 & 0 & 0 & 0 & 2 & 0 & 0 \\
$\begin{array}{l}\text { No. of patients } \\
\text { Multivariable }\end{array}$ & 0 & 0 & 0 & 0 & 193 & 0 & 0 \\
$\begin{array}{l}\text { analysis }(n=6) \\
\text { No. of clusters }\end{array}$ & 21 & & & $\underline{22,24,25}, 74$ & & 35 \\
No. of studies & 1 & 0 & 0 & 2 & 0 & 0 & 1 \\
No. of patients & 32 & 0 & 0 & 309 & 0 & 0 & 92 \\
\hline
\end{tabular}




\begin{tabular}{|c|c|c|c|c|c|c|c|}
\hline \multirow{2}{*}{$\begin{array}{l}\text { Low blood } \\
\text { pressure tendency } \\
(\mathrm{OAG})\end{array}$} & \multicolumn{3}{|c|}{ More progression } & \multirow[b]{2}{*}{ No relation } & \multicolumn{3}{|c|}{ Less progression } \\
\hline & Significant & & $\begin{array}{c}\text { Non- } \\
\text { significant }\end{array}$ & & $\begin{array}{c}\text { Non- } \\
\text { significant }\end{array}$ & & Significant \\
\hline $\begin{array}{l}\text { Univariate } \\
\text { analysis }(n=6)\end{array}$ & & & & $30^{*}, 61$ & $\begin{array}{c}60,77^{*}, \\
\underline{80,81}\end{array}$ & & \\
\hline No. of clusters & 0 & 0 & 0 & 2 & 3 & 0 & 0 \\
\hline No. of studies & 0 & 0 & 0 & 2 & 4 & 0 & 0 \\
\hline No. of patients & 0 & 0 & 0 & 415 & 880 & 0 & 0 \\
\hline $\begin{array}{l}\text { Multivariable } \\
\text { analysis }(n=1)\end{array}$ & & & & 60 & & & \\
\hline No. of clusters & 0 & 0 & 0 & 1 & 0 & 0 & 0 \\
\hline No. of studies & 0 & 0 & 0 & 1 & 0 & 0 & 0 \\
\hline No. of patients & 0 & 0 & 0 & 255 & 0 & 0 & 0 \\
\hline
\end{tabular}

\begin{tabular}{|c|c|c|c|c|c|c|c|}
\hline \multirow{2}{*}{$\begin{array}{l}\text { Duration of } \\
\text { disease (OAG) }\end{array}$} & \multicolumn{3}{|c|}{ More progression } & \multirow[b]{2}{*}{ No relation } & \multicolumn{3}{|c|}{ Less progression } \\
\hline & Significant & & $\begin{array}{c}\text { Non- } \\
\text { significant }\end{array}$ & & $\begin{array}{c}\text { Non- } \\
\text { significant }\end{array}$ & & Significant \\
\hline $\begin{array}{l}\text { Univariate } \\
\text { analysis }(n=5)\end{array}$ & 48 & & 50 & $10^{*}$ & 47,66 & & \\
\hline No. of clusters & 1 & 0 & 1 & 1 & 2 & 0 & 0 \\
\hline No. of studies & 1 & 0 & 1 & 1 & 2 & 0 & 0 \\
\hline No. of patients & 152 & 0 & 88 & 36 & 119 & 0 & 0 \\
\hline $\begin{array}{l}\text { Multivariable } \\
\text { analysis }(n=3)\end{array}$ & & & & 47,66 & 50 & & \\
\hline No. of clusters & 0 & 0 & 0 & 2 & 1 & 0 & 0 \\
\hline No. of studies & 0 & 0 & 0 & 2 & 1 & 0 & 0 \\
\hline No. of patients & 0 & 0 & 0 & 119 & 88 & 0 & 0 \\
\hline
\end{tabular}

\begin{tabular}{|c|c|c|c|c|c|c|c|}
\hline \multirow{2}{*}{$\begin{array}{l}\text { Diurnal IOP } \\
\text { fluctuation (OAG) }\end{array}$} & \multicolumn{3}{|c|}{ More progression } & \multirow[b]{2}{*}{ No relation } & \multicolumn{3}{|c|}{ Less progression } \\
\hline & Significant & & $\begin{array}{c}\text { Non- } \\
\text { significant }\end{array}$ & & $\begin{array}{c}\text { Non- } \\
\text { significant }\end{array}$ & & Significant \\
\hline $\begin{array}{l}\text { Univariate } \\
\text { analysis }(n=2)\end{array}$ & & & & & 35,74 & & \\
\hline No. of clusters & 0 & 0 & 0 & 0 & 2 & 0 & 0 \\
\hline No. of studies & 0 & 0 & 0 & 0 & 2 & 0 & 0 \\
\hline No. of patients & 0 & 0 & 0 & 0 & 193 & 0 & 0 \\
\hline $\begin{array}{l}\text { Multivariable } \\
\text { analysis }(n=3)\end{array}$ & & 22 & 23 & 24 & & & \\
\hline No. of clusters & 0 & 1 & 1 & 1 & 0 & 0 & 0 \\
\hline No. of studies & 0 & 1 & 1 & 1 & 0 & 0 & 0 \\
\hline No. of patients & 0 & 110 & 47 & 70 & 0 & 0 & 0 \\
\hline
\end{tabular}

*Studies comprising a majority of normal-tension glaucoma patients. 


\begin{tabular}{|c|c|c|c|c|c|c|c|}
\hline \multirow{2}{*}{$\begin{array}{l}\text { Mean ocular } \\
\text { perfusion pressure } \\
\text { (OAG) }\end{array}$} & \multicolumn{3}{|c|}{ More progression } & \multirow[b]{2}{*}{ No relation } & \multicolumn{3}{|c|}{ Less progression } \\
\hline & Significant & & $\begin{array}{c}\text { Non- } \\
\text { significant }\end{array}$ & & $\begin{array}{c}\text { Non- } \\
\text { significant }\end{array}$ & & Significant \\
\hline $\begin{array}{l}\text { Univariate } \\
\text { analysis }(n=4)\end{array}$ & & & $74 *$ & 61 & $\underline{72,73}$ & & \\
\hline No. of clusters & 0 & 0 & 1 & 1 & 1 & 0 & 0 \\
\hline No. of studies & 0 & 0 & 1 & 1 & 2 & 0 & 0 \\
\hline No. of patients & 0 & 0 & 101 & 255 & 186 & 0 & 0 \\
\hline $\begin{array}{l}\text { Multivariable } \\
\text { analysis }(n=3)\end{array}$ & & & & $62,74^{*}$ & 73 & & \\
\hline No. of clusters & 0 & 0 & 0 & 2 & 1 & 0 & 0 \\
\hline No. of studies & 0 & 0 & 0 & 2 & 1 & 0 & 0 \\
\hline No. of patients & 0 & 0 & 0 & 356 & 146 & 0 & 0 \\
\hline
\end{tabular}

\begin{tabular}{|c|c|c|c|c|c|c|c|}
\hline \multirow{2}{*}{$\begin{array}{l}\text { Right-hand eye } \\
\text { (OAG) }\end{array}$} & \multicolumn{3}{|c|}{ More progression } & \multirow[b]{2}{*}{ No relation } & \multicolumn{3}{|c|}{ Less progression } \\
\hline & Significant & & $\begin{array}{c}\text { Non- } \\
\text { significant }\end{array}$ & & $\begin{array}{c}\text { Non- } \\
\text { significant }\end{array}$ & & Significant \\
\hline $\begin{array}{l}\text { Univariate } \\
\text { analysis }(n=5)\end{array}$ & & & $\underline{38,40,41}$ & 51 & $32 *$ & & \\
\hline No. of clusters & 0 & 0 & 1 & 1 & 1 & 0 & 0 \\
\hline No. of studies & 0 & 0 & 3 & 1 & 1 & 0 & 0 \\
\hline No. of patients & 0 & 0 & 863 & 40 & 61 & 0 & 0 \\
\hline \multicolumn{8}{|l|}{$\begin{array}{l}\text { Multivariable } \\
\text { analysis }(n=0)\end{array}$} \\
\hline No. of clusters & 0 & 0 & 0 & 0 & 0 & 0 & 0 \\
\hline No. of studies & 0 & 0 & 0 & 0 & 0 & 0 & 0 \\
\hline No. of patients & 0 & 0 & 0 & 0 & 0 & 0 & 0 \\
\hline
\end{tabular}

\begin{tabular}{|c|c|c|c|c|c|}
\hline \multirow{2}{*}{$\begin{array}{l}\text { Lower educational } \\
\text { level (OAG) }\end{array}$} & \multicolumn{2}{|c|}{ More progression } & \multirow[b]{2}{*}{ No relation } & \multicolumn{2}{|c|}{ Less progression } \\
\hline & Significant & $\begin{array}{c}\text { Non- } \\
\text { significant }\end{array}$ & & $\begin{array}{c}\text { Non- } \\
\text { significant }\end{array}$ & Significant \\
\hline
\end{tabular}

\begin{tabular}{|c|c|c|c|c|c|c|c|}
\hline $\begin{array}{l}\text { Univariate } \\
\text { analysis }(n=1)\end{array}$ & & & 41 & & & & \\
\hline No. of clusters & 0 & 0 & 1 & 0 & 0 & 0 & 0 \\
\hline No. of studies & 0 & 0 & 1 & 0 & 0 & 0 & 0 \\
\hline No. of patients & 0 & 0 & 401 & 0 & 0 & 0 & 0 \\
\hline $\begin{array}{l}\text { Multivariable } \\
\text { analysis }(n=4)\end{array}$ & & & 38 & 39,55 & 41 & & \\
\hline No. of clusters & 0 & 0 & 1 & 2 & 1 & 0 & 0 \\
\hline No. of studies & 0 & 0 & 1 & 2 & 1 & 0 & 0 \\
\hline No. of patients & 0 & 0 & 301 & 1165 & 401 & 0 & 0 \\
\hline
\end{tabular}

*Studies comprising a majority of normal-tension glaucoma patients. 


\begin{tabular}{|c|c|c|c|c|c|c|c|}
\hline \multirow{2}{*}{$\begin{array}{l}\text { Minimum IOP of } \\
\text { diurnal variation } \\
\text { (OAG) }\end{array}$} & \multicolumn{3}{|c|}{ More progression } & \multirow[b]{2}{*}{ No relation } & \multicolumn{3}{|c|}{ Less progression } \\
\hline & Significant & & $\begin{array}{c}\text { Non- } \\
\text { significant }\end{array}$ & & $\begin{array}{c}\text { Non- } \\
\text { significant }\end{array}$ & & Significant \\
\hline $\begin{array}{l}\text { Univariate } \\
\text { analysis }(n=2)\end{array}$ & & & & & 35,74 & & \\
\hline No. of clusters & 0 & 0 & 0 & 0 & 2 & 0 & 0 \\
\hline No. of studies & 0 & 0 & 0 & 0 & 2 & 0 & 0 \\
\hline No. of patients & 0 & 0 & 0 & 0 & 193 & 0 & 0 \\
\hline $\begin{array}{l}\text { Multivariable } \\
\text { analysis }(n=2)\end{array}$ & & & & $\underline{22,24}$ & & & \\
\hline No. of clusters & 0 & 0 & 0 & 1 & 0 & 0 & 0 \\
\hline No. of studies & 0 & 0 & 0 & 2 & 0 & 0 & 0 \\
\hline No. of patients & 0 & 0 & 0 & 180 & 0 & 0 & 0 \\
\hline
\end{tabular}

\begin{tabular}{|c|c|c|c|c|c|c|c|}
\hline \multirow{2}{*}{$\begin{array}{l}\text { Maximum IOP of } \\
\text { diurnal variation } \\
\text { (OAG) }\end{array}$} & \multicolumn{3}{|c|}{ More progression } & \multirow[b]{2}{*}{ No relation } & \multicolumn{3}{|c|}{ Less progression } \\
\hline & Significant & & $\begin{array}{c}\text { Non- } \\
\text { significant }\end{array}$ & & $\begin{array}{c}\text { Non- } \\
\text { significant }\end{array}$ & & Significant \\
\hline $\begin{array}{l}\text { Univariate } \\
\text { analysis }(n=2)\end{array}$ & & & & & 35,74 & & \\
\hline No. of clusters & 0 & 0 & 0 & 0 & 2 & 0 & 0 \\
\hline No. of studies & 0 & 0 & 0 & 0 & 2 & 0 & 0 \\
\hline No. of patients & 0 & 0 & 0 & 0 & 193 & 0 & 0 \\
\hline $\begin{array}{l}\text { Multivariable } \\
\text { analysis }(n=2)\end{array}$ & & & & $\underline{22,24}$ & & & \\
\hline No. of clusters & 0 & 0 & 0 & 1 & 0 & 0 & 0 \\
\hline No. of studies & 0 & 0 & 0 & 2 & 0 & 0 & 0 \\
\hline No. of patients & 0 & 0 & 0 & 180 & 0 & 0 & 0 \\
\hline
\end{tabular}

\begin{tabular}{|c|c|c|c|c|c|c|c|}
\hline \multirow{2}{*}{$\begin{array}{l}\text { Major hypoten- } \\
\text { sion (OAG) }\end{array}$} & \multicolumn{3}{|c|}{ More progression } & \multirow[b]{2}{*}{ No relation } & \multicolumn{3}{|c|}{ Less progression } \\
\hline & Significant & & $\begin{array}{c}\text { Non- } \\
\text { significant }\end{array}$ & & $\begin{array}{c}\text { Non- } \\
\text { significant }\end{array}$ & & Significant \\
\hline $\begin{array}{l}\text { Univariate } \\
\text { analysis }(n=3)\end{array}$ & & & $26^{*}$ & $30 *$ & $76^{*}$ & & \\
\hline No. of clusters & 0 & 0 & 1 & 1 & 1 & 0 & 0 \\
\hline No. of studies & 0 & 0 & 1 & 1 & 1 & 0 & 0 \\
\hline No. of patients & 0 & 0 & 59 & 160 & 286 & 0 & 0 \\
\hline $\begin{array}{l}\text { Multivariable } \\
\text { analysis }(n=1)\end{array}$ & & & & 62 & & & \\
\hline No. of clusters & 0 & 0 & 0 & 1 & 0 & 0 & 0 \\
\hline No. of studies & 0 & 0 & 0 & 1 & 0 & 0 & 0 \\
\hline No. of patients & 0 & 0 & 0 & 255 & 0 & 0 & 0 \\
\hline
\end{tabular}

*Studies comprising a majority of normal-tension glaucoma patients. 


\begin{tabular}{|c|c|c|c|c|c|c|c|}
\hline \multirow{2}{*}{$\begin{array}{l}\text { Older age at } \\
\text { diagnosis (OAG) }\end{array}$} & \multicolumn{3}{|c|}{ More progression } & \multirow[b]{2}{*}{ No relation } & \multicolumn{3}{|c|}{ Less progression } \\
\hline & Significant & & $\begin{array}{c}\text { Non- } \\
\text { significant }\end{array}$ & & $\begin{array}{c}\text { Non- } \\
\text { significant }\end{array}$ & & Significant \\
\hline $\begin{array}{l}\text { Univariate } \\
\text { analysis }(n=3)\end{array}$ & & & 47 & 50 & 48 & & \\
\hline No. of clusters & 0 & 0 & 1 & 1 & 1 & 0 & 0 \\
\hline No. of studies & 0 & 0 & 1 & 1 & 1 & 0 & 0 \\
\hline No. of patients & 0 & 0 & 48 & 88 & 152 & 0 & 0 \\
\hline $\begin{array}{l}\text { Multivariable } \\
\text { analysis }(n=2)\end{array}$ & & & & 47 & 50 & & \\
\hline No. of clusters & 0 & 0 & 0 & 1 & 1 & 0 & 0 \\
\hline No. of studies & 0 & 0 & 0 & 1 & 1 & 0 & 0 \\
\hline No. of patients & 0 & 0 & 0 & 48 & 88 & 0 & 0 \\
\hline
\end{tabular}

\begin{tabular}{|c|c|c|c|c|c|c|c|}
\hline \multirow{2}{*}{$\begin{array}{l}\text { Mean arterial } \\
\text { blood pressure } \\
\text { (OAG) }\end{array}$} & \multicolumn{3}{|c|}{ More progression } & \multirow[b]{2}{*}{ No relation } & \multicolumn{3}{|c|}{ Less progression } \\
\hline & Significant & & $\begin{array}{c}\text { Non- } \\
\text { significant }\end{array}$ & & $\begin{array}{c}\text { Non- } \\
\text { significant }\end{array}$ & & Significant \\
\hline $\begin{array}{l}\text { Univariate } \\
\text { analysis }(n=2)\end{array}$ & & & $74 *$ & & & & 73 \\
\hline No. of clusters & 0 & 0 & 1 & 0 & 0 & 0 & 1 \\
\hline No. of studies & 0 & 0 & 1 & 0 & 0 & 0 & 1 \\
\hline No. of patients & 0 & 0 & 101 & 0 & 0 & 0 & 146 \\
\hline $\begin{array}{l}\text { Multivariable } \\
\text { analysis }(n=2)\end{array}$ & & & & $21^{*}$ & 73 & & \\
\hline No. of clusters & 0 & 0 & 0 & 1 & 1 & 0 & 0 \\
\hline No. of studies & 0 & 0 & 0 & 1 & 1 & 0 & 0 \\
\hline No. of patients & 0 & 0 & 0 & 32 & 146 & 0 & 0 \\
\hline
\end{tabular}

\begin{tabular}{|c|c|c|c|c|c|c|c|}
\hline \multirow[b]{2}{*}{ Disc size (OAG) } & \multicolumn{3}{|c|}{ More progression } & \multirow[b]{2}{*}{ No relation } & \multicolumn{3}{|c|}{ Less progression } \\
\hline & Significant & & $\begin{array}{c}\text { Non- } \\
\text { significant }\end{array}$ & & $\begin{array}{c}\text { Non- } \\
\text { significant }\end{array}$ & & Significant \\
\hline $\begin{array}{l}\text { Univariate } \\
\text { analysis }(n=3)\end{array}$ & & & 13,82 & $30 *$ & & & \\
\hline No. of clusters & 0 & 0 & 2 & 1 & 0 & 0 & 0 \\
\hline No. of studies & 0 & 0 & 2 & 1 & 0 & 0 & 0 \\
\hline No. of patients & 0 & 0 & 275 & 160 & 0 & 0 & 0 \\
\hline $\begin{array}{l}\text { Multivariable } \\
\text { analysis }(n=1)\end{array}$ & & & & 13 & & & \\
\hline No. of clusters & 0 & 0 & 0 & 1 & 0 & 0 & 0 \\
\hline No. of studies & 0 & 0 & 0 & 1 & 0 & 0 & 0 \\
\hline No. of patients & 0 & 0 & 0 & 30 & 0 & 0 & 0 \\
\hline
\end{tabular}

*Studies comprising a majority of normal-tension glaucoma patients. 


\begin{tabular}{|c|c|c|c|c|c|c|c|}
\hline \multirow{2}{*}{$\begin{array}{l}\text { Threat to fixation } \\
\text { (OAG) }\end{array}$} & \multicolumn{3}{|c|}{ More progression } & \multirow[b]{2}{*}{ No relation } & \multicolumn{3}{|c|}{ Less progression } \\
\hline & Significant & & $\begin{array}{c}\text { Non- } \\
\text { significant }\end{array}$ & & $\begin{array}{c}\text { Non- } \\
\text { significant }\end{array}$ & & Significant \\
\hline $\begin{array}{l}\text { Univariate } \\
\text { analysis }(n=3)\end{array}$ & & & & 4,65 & 80 & & \\
\hline No. of clusters & 0 & 0 & 0 & 2 & 1 & 0 & 0 \\
\hline No. of studies & 0 & 0 & 0 & 2 & 1 & 0 & 0 \\
\hline No. of patients & 0 & 0 & 0 & 134 & 293 & 0 & 0 \\
\hline \multicolumn{8}{|l|}{$\begin{array}{l}\text { Multivariable } \\
\text { analysis }(n=0)\end{array}$} \\
\hline No. of clusters & 0 & 0 & 0 & 0 & 0 & 0 & 0 \\
\hline No. of studies & 0 & 0 & 0 & 0 & 0 & 0 & 0 \\
\hline No. of patients & 0 & 0 & 0 & 0 & 0 & 0 & 0 \\
\hline
\end{tabular}

\begin{tabular}{|c|c|c|c|c|c|c|c|}
\hline \multirow{2}{*}{$\begin{array}{l}\text { Cardiac arrhyth- } \\
\text { mia (OAG) }\end{array}$} & \multicolumn{3}{|c|}{ More progression } & \multirow[b]{2}{*}{ No relation } & \multicolumn{3}{|c|}{ Less progression } \\
\hline & Significant & & $\begin{array}{c}\text { Non- } \\
\text { significant }\end{array}$ & & $\begin{array}{c}\text { Non- } \\
\text { significant }\end{array}$ & & Significant \\
\hline $\begin{array}{l}\text { Univariate } \\
\text { analysis }(n=3)\end{array}$ & 76 & & 77 & 30 & & & \\
\hline No. of clusters & 1 & 0 & 1 & 1 & 0 & 0 & 0 \\
\hline No. of studies & 1 & 0 & 1 & 1 & 0 & 0 & 0 \\
\hline No. of patients & 286 & 0 & 256 & 160 & 0 & 0 & 0 \\
\hline $\begin{array}{l}\text { Multivariable } \\
\text { analysis }(n=1)\end{array}$ & & & & 76 & & & \\
\hline No. of clusters & 0 & 0 & 0 & 1 & 0 & 0 & 0 \\
\hline No. of studies & 0 & 0 & 0 & 1 & 0 & 0 & 0 \\
\hline No. of patients & 0 & 0 & 0 & 286 & 0 & 0 & 0 \\
\hline
\end{tabular}

\begin{tabular}{|c|c|c|c|c|c|c|c|}
\hline \multirow{2}{*}{$\begin{array}{l}\text { High cholesterol } \\
\text { levels (OAG) }\end{array}$} & \multicolumn{3}{|c|}{ More progression } & \multirow[b]{2}{*}{ No relation } & \multicolumn{3}{|c|}{ Less progression } \\
\hline & Significant & & $\begin{array}{c}\text { Non- } \\
\text { significant }\end{array}$ & & $\begin{array}{c}\text { Non- } \\
\text { significant }\end{array}$ & & Significant \\
\hline $\begin{array}{l}\text { Univariate } \\
\text { analysis }(n=3)\end{array}$ & & & & 27 & $76^{*}$ & & $77^{*}$ \\
\hline No. of clusters & 0 & 0 & 0 & 1 & 1 & 0 & 1 \\
\hline No. of studies & 0 & 0 & 0 & 1 & 1 & 0 & 1 \\
\hline No. of patients & 0 & 0 & 0 & 70 & 286 & 0 & 256 \\
\hline $\begin{array}{l}\text { Multivariable } \\
\text { analysis }(n=1)\end{array}$ & & & & $77^{*}$ & & & \\
\hline No. of clusters & 0 & 0 & 0 & 1 & 0 & 0 & 0 \\
\hline No. of studies & 0 & 0 & 0 & 1 & 0 & 0 & 0 \\
\hline No. of patients & 0 & 0 & 0 & 256 & 0 & 0 & 0 \\
\hline
\end{tabular}

*Studies comprising a majority of normal-tension glaucoma patients. 


\begin{tabular}{|c|c|c|c|c|c|c|c|}
\hline \multirow{2}{*}{$\begin{array}{l}\text { Focal ischemic } \\
\text { disc pathology } \\
\text { (OAG) }\end{array}$} & \multicolumn{3}{|c|}{ More progression } & \multirow[b]{2}{*}{ No relation } & \multicolumn{3}{|c|}{ Less progression } \\
\hline & Significant & & $\begin{array}{c}\text { Non- } \\
\text { significant }\end{array}$ & & $\begin{array}{c}\text { Non- } \\
\text { significant }\end{array}$ & & Significant \\
\hline $\begin{array}{l}\text { Univariate } \\
\text { analysis }(n=1)\end{array}$ & & & 45 & & & & \\
\hline No. of clusters & 0 & 0 & 1 & 0 & 0 & 0 & 0 \\
\hline No. of studies & 0 & 0 & 1 & 0 & 0 & 0 & 0 \\
\hline No. of patients & 0 & 0 & 72 & 0 & 0 & 0 & 0 \\
\hline $\begin{array}{l}\text { Multivariable } \\
\text { analysis }(n=2)\end{array}$ & & & & $\underline{22 *, 24 *}$ & & & \\
\hline No. of clusters & 0 & 0 & 0 & 1 & 0 & 0 & 0 \\
\hline No. of studies & 0 & 0 & 0 & 2 & 0 & 0 & 0 \\
\hline No. of patients & 0 & 0 & 0 & 180 & 0 & 0 & 0 \\
\hline
\end{tabular}

*Studies comprising a majority of normal-tension glaucoma patients. 



\section{CHAPTER 3}

\section{Prediction of glaucomatous visual field progression using baseline clinical data}

Authors:

Paul J.G. Ernest

Jan S.A.G. Schouten

Henny J.M. Beckers

Fred Hendrikse

Martin H. Prins

Carroll A.B. Webers

Journal of Glaucoma (published ahead-of-print) 


\section{ABSTRACT}

Purpose: To develop a prediction model for glaucomatous visual field progression using easily accessible baseline clinical data.

Patients and Methods: We collected baseline data of 613 consecutive patients with open-angle glaucoma from 2001 to 2003. The rate of visual field progression was calculated using the Visual Field Index (VFI) of routine follow-up examinations until 2010. Baseline data of 333 patients from three hospitals were used to develop a model to predict the rate of VFI progression using a linear regression analysis and univariate pre-selection $(p<0.1)$ of eight candidate predictors. The performance of the model was investigated using $R^{2}$, the area under the receiver operating characteristic curve (AUC), and calibration plots. The prediction model was internally validated using bootstrapping and externally validated in 280 patients from two other hospitals.

Results: After a mean follow-up period of 5.8 years of all 613 eyes, the mean rate of VFI progression was $-1.6 \%$ per year. During model development, $10.3 \%$ of the observed variation in VFI rates was explained by the model. The AUC was 0.76 when the prediction model was used to detect a VFI rate $\leq-3 \%$ per year, which decreased to 0.71 at external validation. The final model contained the following predictors: age, baseline intraocular pressure (IOP), and baseline visual field status.

Conclusions: Our prediction model may offer the possibility to indentify subgroups of treated patients with high rates of visual field progression, thereby providing an opportunity for more intensive treatment of those high-risk glaucoma patients. 


\section{Introduction}

Open-angle glaucoma (OAG) is a chronic, progressive optic neuropathy with a characteristic appearance of the optic nerve and associated visual field defects. ${ }^{1}$ Glaucoma is the second leading cause of blindness worldwide. ${ }^{2}$ The ability to differentiate rapidly progressing patients from slowly progressing patients would give ophthalmologists the opportunity to direct more intensive treatment to those who are at a high risk of becoming blind, and could additionally prevent adverse events and costs of intensive treatment in those at a low risk of blindness. ${ }^{3}$ One approach to differentiate between patients with fast and slow progression is to monitor disease progression. However, this consumes time and damage has already occurred before treatment is intensified. Another approach is to predict progression.

Currently, an ophthalmologist takes into account the patient's life expectancy and disease stage to estimate the patient's risk of visual disability. A reliable rate of visual field progression should also be estimated for this purpose. ${ }^{4}$ Knowledge of prognostic factors (i.e. predictors of progression) can help the ophthalmologist to identify patients at risk of high rates of progression. Combining prognostic factors in a clinical prediction model may further quantify the risk and makes it easier for a clinician to aggregate knowledge of several prognostic factors. ${ }^{5,6}$ However, currently there is no model available to predict the rate of glaucomatous visual field progression.

The aim of the present study is to develop a model to predict the rate of visual field progression in individual OAG patients by quantifying the contribution of several baseline prognostic factors.

\section{Material and methods}

\section{Study population}

We performed a retrospective analysis based on data from a multicentre prospective follow-up study. We used the database of the DUtch Research project on treatment outcome IN Glaucoma patients (DURING) study to identify OAG patients who were consecutively recruited in the latter project from 2001 to 2003. ${ }^{7,8}$ All patients were treated with topical glaucoma medication at baseline. Patients received routine followup examinations and standard medical care prescribed by their ophthalmologist. Informed consent was obtained from all patients. Seventy-nine percent of the invited patients eventually participated in the DURING study. For the current study we collected patients from five out of the nine originally participating Dutch hospitals, including 
one academic hospital. This study was approved by the local ethics committees and conformed to the tenets of the Declaration of Helsinki. All patients gave their informed consent prior to inclusion in the study.

A flow chart of the patient selection process is shown in Figure 1. We used 333 patients from three hospitals to develop the prediction model (i.e. the development sample in Figure 1a) and 280 patients from two other hospitals to validate the model (i.e. the validation sample in Figure $1 \mathrm{~b}$ ). We included one eye of each patient. OAG had to be diagnosed in the study eye by the ophthalmologist at the baseline visit of the DURING study. Eyes diagnosed as having primary open-angle glaucoma (POAG), normal tension glaucoma (NTG), pigment glaucoma (PG), or pseudoexfoliation glaucoma (PXG) were included.

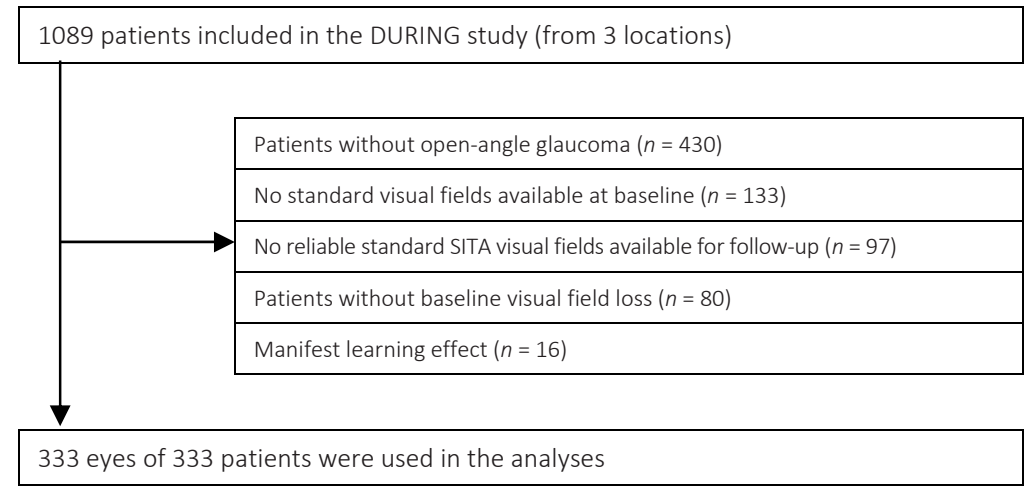

Figure 1a: Flow chart of the patient selection process for the development sample

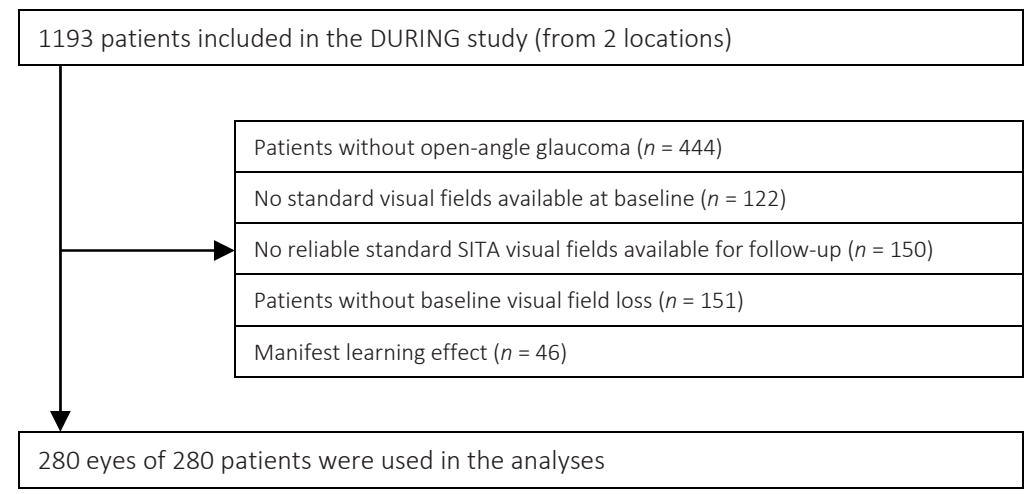

Figure $1 \mathrm{~b}$ : Flow chart of the patient selection process for the validation sample DURING = DUtch Research project on treatment outcome IN Glaucoma patients, SITA = Swedish Interactive Thresholding Algorithm. 


\section{Visual fields}

All visual fields in this study were performed with the Humphrey Field Analyzer (HFA) (Carl Zeiss Meditec, Dublin, CA, USA). Standard 24-2 or 30-2 visual fields obtained earlier or at the same day of the baseline visit of the DURING study had to be available for each patient for inclusion (Figure 1). At least two standard Swedish Interactive Thresholding Algorithm (SITA) Fast or SITA Standard visual field tests with less than 15\% false positive answers were required. Baseline visual field loss was required for each included eye. This was indicated by a Glaucoma Hemifield Test (GHT) "outside normal limits" and/or a significant $(p<0.05)$ deviation of the pattern standard deviation index (PSD). If both eyes were diagnosed as having OAG, we selected the most affected eye based on the GHT and the PSD. If both eyes of a patient were equally affected, one eye was randomly chosen. No manifest learning effect was permitted for patients with only two available visual fields (see Figure 1).

All available visual fields were collected until 2010 and the Visual Field Index (VFI) was extracted. The VFI is a summary value of all individual test points in one visual field and is expressed as a simple percentage, $100 \%$ representing a normal visual field. Although the VFI may not be suited to detect early visual field damage and has not proven to be superior to the mean deviation (MD) index, ${ }^{9}$ we chose the rate of the VFI as measure of visual field progression, because the VFI is corrected for the effect of cataract, is more heavily weighted to central areas of the visual field, and is used to calculate a patient's rate of progression in modern HFA software, expressed as percentage of visual field loss per year. ${ }^{10}$

\section{Candidate predictors}

The following eight candidate predictors, which were available at the baseline visit of the DURING study, were included in the initial prediction model: gender, age, mean intraocular pressure (IOP) of the last two visits at baseline, MD value of the baseline visual field, diagnostic category of $O A G$, previous disease progression subjectively determined by the ophthalmologist, patient's adherence with medication, and the MD difference between the study eye and the fellow eye. IOP and baseline MD were initially modeled as continuous variables but were recoded as categorical predictors based on hypotheses of a nonlinear relation with progression. ${ }^{11,12}$ The effect on $R^{2}$ was assessed and the coding that explained most of the variance was used for the multivariable model. Disease progression and adherence were derived from a form filled in by ophthalmologists participating in the DURING study at the baseline visit. One of the questions was "Are you changing therapy?". If the answer was "yes", he/she was asked why the therapy was changed. Possible answers were "the current therapy has no/little effect on IOP", "target pressure has not been reached", "adverse effects", or "because 
of glaucoma progression". The patients whose therapy was changed because of glaucoma progression were labeled progressive at baseline for the current study. Adherence was assessed by the question "When was the last time the patient put drops in his eyes?". Generally, glaucoma eye drops have to be used at least once every 24 hours. We therefore labeled the patient as being non-adherent for the current study if the answer was "before yesterday morning".

\section{Model development}

In the development sample from three of the five hospitals $(n=333)$, we applied a multivariable linear regression analysis to develop a model with variables that predict the $\mathrm{VFI}$ rate over the follow-up period. We applied a univariate pre-selection of the eight candidate predictors with linear regression analysis and used $p<0.10$ to include predictors in the multivariable model. As a control analysis, we also applied a forward and backward selection approach with an entry criterion of $\mathrm{p}<0.05$ and a removal criterion of $p<0.10$ respectively.

When patients had missing values for predictors in the univariate analysis, we performed a complete case analysis (i.e. we only included patients with available data in the specific analysis). The influence of the following five potentially confounding factors was assessed by alternatively adding these factors to the multivariable model: duration of follow-up, previous experience with perimetry, number of visual field assessments performed, clinical centre where the patient was included, and whether the patient was already treated before the baseline visit.

\section{Model evaluation}

The performance of the model was expressed in terms of explained variation $\left(R^{2}\right.$ statistic). Furthermore, the discriminative ability of the model was tested by calculating the areas under the receiver operating characteristic (ROC) curves (AUC) for detecting rates of progression from $-1 \%$ to $-4 \%$ per year. An AUC near 0.5 indicates that the model is no better than a coin-flip in identifying progressive patients and a value of 1.0 indicates a perfect test. ${ }^{13}$ We also made contingency tables and calculated the sensitivity, specificity, and predictive values for different cut-off points on the ROC curves. A calibration plot was constructed to make a visual evaluation of the fit of the model in the study population.

The clinical prediction model was internally validated using 200 bootstrap samples. ${ }^{14}$ We calculated optimism-corrected performance measures. We also calculated the optimism-corrected calibration slope and used this as a uniform shrinkage factor for the regression coefficients in the multivariable model. ${ }^{15}$ After shrinkage, we calculated the new regression constant by making the mean predicted and observed VFI rate equal. 
The clinical prediction model was externally validated in a validation sample derived from the two other hospitals $(n=280)$. We applied the internally validated model in this sample to predict individual VFI rates. The AUC was calculated and a new calibration plot was constructed. We also fitted the prediction model in the validation sample, compared the new regression coefficients with the coefficients in the development sample, and calculated the new $R^{2}$. We eventually formulated the final regression formula based on the entire sample derived from all hospitals ( $n=613$ ).

Internal model validation was performed using the statistical software R (version 2.9.1). For all other analyses we used SPSS for Windows (version 15.0).

\section{Results}

\section{Study population}

We included 613 eyes of 613 patients. After a mean follow-up period of 5.8 years, a mean of 5.0 visual fields were performed. The mean rate of VFI progression was $-1.6 \%$ per year in the entire population. We constructed the prediction model based on 333 patients from hospitals A, B, and C. The prediction model was externally validated in 280 patients from hospitals $D$ and E. Table 1 summarizes the descriptive statistics of the candidate predictors, the potentially confounding variables, and the outcome measure for both groups. For the development sample, the mean observed rate of VFI progression was $-1.9 \%$ (standard deviation (SD) \pm 3.4 ) per year. The validation sample had a lower mean rate of VFI progression of $-1.3 \%$ (SD \pm 3.2 ) per year (Table 1 ). Values for the candidate predictors were comparable between both groups, although the validation sample had a better baseline visual field (Table 1).

\section{Model development}

After categorizing the continuous predictor baseline IOP into IOP $\leq 21 \mathrm{mmHg}$ and IOP $>21 \mathrm{mmHg}$, the univariate model $R^{2}$ improved from $1.1 \%$ to $2.6 \%$. This cut-off point also had the highest $R^{2}$ when we varied the cut-off points in the univariate analysis from 18 to $26 \mathrm{mmHg}$. Dividing IOP into tertiles did not improve the model. Categorizing the continuous predictor baseline MD into tertiles led to an improvement from $1.2 \%$ to $4.0 \%$ in the univariate model $R^{2}$. The group with the best visual fields was selected as reference group for the groups with moderate visual field loss (i.e. MD values $\leq-4.6 \mathrm{~dB}$ and $>-10.8 \mathrm{~dB}$ ) and severe visual field loss (i.e. MD values $\leq-10.8 \mathrm{~dB}$ ). The model did not further improve when we categorized baseline MD into quartiles. 
Age, IOP and baseline visual field status were entered into the multivariable model after univariate pre-selection of predictors (Table 2). No confounding variables had considerable effects on the regression coefficients or the model $R^{2}$ except baseline experience with perimetry. The $R^{2}$ of the multivariable model changed from $8.7 \%$ to $11.2 \%$ when a dichotomous variable was included that indicated patients with a lack of previous perimetric experience as well as only two available visual fields. Therefore, we decided to exclude these 16 patients (i.e. indicated by "manifest learning effect" in Figure 1). Twenty-five patients with two available visual fields and previous perimetric experience remained in the population of 333 patients. Without these patients, $R^{2}$ of the multivariable model changed from $10.3 \%$ to $10.6 \%$. We decided to keep these patients in the model. With a forward selection approach the same multivariable model was obtained. With backward selection, the multivariable model contained adherence as extra predictor $(p=0.064)$. The positive regression coefficient (i.e. 1.853) means that non-adherence results in less visual field progression. Because of its borderline statistical significance and contra intuitive direction of its relation with progression, we did not include this variable in the multivariable model. 
Table 1: Descriptive statistics of the study population

\begin{tabular}{|c|c|c|}
\hline Descriptive statistics & $\begin{array}{l}\text { Development sample } \\
\qquad(n=333)\end{array}$ & $\begin{array}{c}\text { Validation sample } \\
\qquad(n=280)\end{array}$ \\
\hline \multicolumn{3}{|l|}{ Candidate predictors } \\
\hline Female sex & $130(39 \%)$ & 138 (49\%) \\
\hline Age & 68.1 years $( \pm 10.9)$ & 69.1 years $( \pm 9.0)$ \\
\hline Baseline IOP & $16.4 \mathrm{mmHg}( \pm 3.8)$ & $16.2 \mathrm{mmHg}( \pm 3.5)$ \\
\hline Low IOP ( $\leq 21$ mmHg) & 295 (89\%) & 262 (94\%) \\
\hline High IOP (>21 mmHg) & $38(11 \%)$ & $18(6 \%)$ \\
\hline Baseline MD & $-8.9 \mathrm{~dB}( \pm 6.8)$ & $-7.2 \mathrm{~dB}( \pm 6.6)$ \\
\hline High MD (>-4.6 dB) & $111(33 \%)$ & $127(45 \%)$ \\
\hline Moderate MD $(-10.8 \mathrm{~dB}<\mathrm{MD} \leq-4.6 \mathrm{~dB})$ & $112(34 \%)$ & 87 (31\%) \\
\hline Low MD (s-10.8 dB) & $110(33 \%)$ & $66(24 \%)$ \\
\hline \multicolumn{3}{|l|}{ Diagnosis } \\
\hline POAG & $280(84 \%)$ & 257 (92\%) \\
\hline NTG & $34(10 \%)$ & $18(6 \%)$ \\
\hline PG & $13(4 \%)$ & $2(1 \%)$ \\
\hline PXG & $6(2 \%)$ & $3(1 \%)$ \\
\hline Subjective progression ${ }^{\mathrm{a}}$ & $19(6 \%)$ & $3(1 \%)$ \\
\hline Last eye drop $\geq 2$ days ago ${ }^{b}$ & $11(3 \%)$ & $3(1 \%)$ \\
\hline MD difference between both eyes ${ }^{c}$ & $-2.5 \mathrm{~dB}( \pm 8.5)$ & $-3.0 \mathrm{~dB}( \pm 7.1)$ \\
\hline \multicolumn{3}{|l|}{ Potentially confounding variables } \\
\hline Follow-up period & 6.2 years $( \pm 2.4)$ & 5.3 years $( \pm 2.2)$ \\
\hline Previous perimetric experience & $266(80 \%)$ & $200(71 \%)$ \\
\hline Number of visual fields & $5.9( \pm 2.7)$ & $4.0( \pm 1.7)$ \\
\hline \multicolumn{3}{|l|}{ Clinical centre } \\
\hline A & $230(69 \%)$ & - \\
\hline B & $39(12 \%)$ & - \\
\hline C & $64(19 \%)$ & - \\
\hline D & - & $93(33 \%)$ \\
\hline$E$ & - & $187(67 \%)$ \\
\hline Patients not treated before baseline & $7(2 \%)$ & $10(4 \%)$ \\
\hline \multicolumn{3}{|l|}{ Outcome variable } \\
\hline Observed VFI rate & $-1.9 \%( \pm 3.4)$ & $-1.3 \%( \pm 3.2)$ \\
\hline
\end{tabular}

Values are displayed as means and standard deviations or frequencies and percentages.

${ }^{a}$ Complete case analysis with 330 patients and 277 patients respectively.

${ }^{\mathrm{b}}$ Complete case analysis with 315 patients and 267 patients respectively.

${ }^{c}$ Complete case analysis with 278 patients and 182 patients respectively; a positive value indicates a worse fellow eye.

$\mathrm{IOP}=$ intraocular pressure, $\mathrm{MD}=$ mean deviation, $\mathrm{POAG}=$ primary open-angle glaucoma, $\mathrm{NTG}=$ normaltension glaucoma, $\mathrm{PG}=$ pigment glaucoma, $\mathrm{PXG}=$ pseudoexfoliation glaucoma, $\mathrm{VFI}=$ Visual Field Index. 
Table 2: Results of univariate pre-selection of predictors

\begin{tabular}{|c|c|c|c|c|}
\hline \multirow{2}{*}{ Candidate predictors } & \multicolumn{4}{|c|}{ Coefficients and model fit } \\
\hline & Beta & Confidence interval & Significance & $R^{2}$ \\
\hline $\begin{array}{l}\text { Moderate visual field group } \\
\text { (ref. best visual field group) }\end{array}$ & -1.31 & $-2.18,-0.44$ & 0.003 & $4.4 \%$ \\
\hline $\begin{array}{l}\text { Poor visual field group } \\
\text { (ref. best visual field group) }\end{array}$ & -1.63 & $-2.51,-0.76$ & 0.000 & $4.4 \%$ \\
\hline $\begin{array}{l}\text { High IOP (>21 mmHg) } \\
\text { (ref. low IOP) }\end{array}$ & -1.93 & $-3.05,-0.80$ & 0.001 & $3.3 \%$ \\
\hline $\begin{array}{l}\text { Age } \\
\text { (per year older) }\end{array}$ & -0.05 & $-0.08,-0.01$ & 0.005 & $2.3 \%$ \\
\hline $\begin{array}{l}\text { Female sex } \\
\text { (ref. male sex) }\end{array}$ & -0.58 & $-1.32,0.17$ & 0.127 & $0.7 \%$ \\
\hline $\begin{array}{l}\text { MD difference in both eyes }{ }^{a} \\
\text { (per decibel higher) }\end{array}$ & 0.03 & $-0.01,0.08$ & 0.176 & $0.7 \%$ \\
\hline $\begin{array}{l}\text { Last eye drop } \geq 2 \text { days ago }{ }^{\text {b }} \\
\text { (ref. }<2 \text { days ago) }\end{array}$ & 1.43 & $-0.57,3.43$ & 0.161 & $0.6 \%$ \\
\hline $\begin{array}{l}\text { Normal-tension glaucoma } \\
\text { (ref. POAG) }\end{array}$ & 0.29 & $-0.92,1.50$ & 0.636 & $0.5 \%$ \\
\hline $\begin{array}{l}\text { Pigment glaucoma } \\
\text { (ref. POAG) }\end{array}$ & -0.96 & $-2.85,0.92$ & 0.315 & $0.5 \%$ \\
\hline $\begin{array}{l}\text { Pseudoexfoliation glaucoma } \\
\text { (ref. POAG) }\end{array}$ & 0.91 & $-1.83,3.65$ & 0.516 & $0.5 \%$ \\
\hline $\begin{array}{l}\text { Subjective progression }{ }^{c} \\
\text { (ref. no progression) }\end{array}$ & 0.71 & $-0.86,2.28$ & 0.376 & $0.2 \%$ \\
\hline
\end{tabular}

Results of the univariate analyses for 333 eyes of 333 patients. The candidate predictors are ranked according to their $R^{2}$ value. Age, IOP and baseline visual field status were eventually selected for the multivariable analysis.

${ }^{a}$ Complete case analysis with 278 patients; a positive value indicates a worse fellow eye.

${ }^{\mathrm{b}}$ Complete case analysis with 315 patients.

${ }^{\mathrm{c}}$ Complete case analysis with 330 patients.

$R^{2}=$ explained variation, which is a measure of the model fit, ref. = reference group, $I O P=$ intraocular pressure, $\mathrm{MD}=$ mean deviation, $\mathrm{POAG}=$ primary open-angle glaucoma. 
The multivariable model contained the following predictors and accompanying regression coefficients: age (-0.04\% per year older), baseline IOP

$(-2.18 \%$ for IOP values above $21 \mathrm{mmHg})$, and baseline visual field status $(-1.34 \%$ for moderate and $-1.71 \%$ for severely affected visual fields).

\section{Model evaluation in the development sample}

$R^{2}$ of the multivariable model was $10.3 \%$. The calibration plot is shown in Figure $2 a$. The mean absolute difference between the observed and predicted VFI rate was $1.97 \%$ and had a positively skewed distribution.

Based on the relatively large scattering in the calibration plot and the relatively high absolute difference between the observed and predicted VFI rate, we assessed the value of the model in identifying rapidly progressing patients instead of predicting an exact VFI rate for individual patients. Therefore, we calculated the AUC for detecting different rates of visual field progression (Table 3). The highest AUC (i.e. 0.76) was found when the model was used to detect a rate $\leq-3 \%$ per year. The ROC curve for this rate is shown in Figure 3. Seventy-five out of 333 patients (22.5\%) had an observed VFI rate $\leq-3 \%$ per year. We calculated the sensitivity, specificity, and predictive values for different cut-off points on this ROC curve (Table 4). When using a cut-off point of $-3.0 \%$ on the ROC curve, we found a positive predictive value (PPV) of 0.61 and a negative predictive value (NPV) of 0.81 (Table 4). This corresponds to a $61 \%$ and $19 \%$ chance of being rapidly progressive with a positive and negative test result, respectively.

Using 200 bootstrap samples, we estimated the optimism-corrected $R^{2}$ at $7.2 \%$ and the AUC at 0.75 for the detection of a rate $\leq-3 \%$ per year. The uniform shrinkage factor estimated with bootstrapping was 0.925 , resulting in the following internally validated model:

VFI rate $=$

$2.05+-0.04 *$ age $+-2.01 *$ IOP $>21 \mathrm{mmHg}+-1.24 *$ moderate MD + -1.58* low MD 


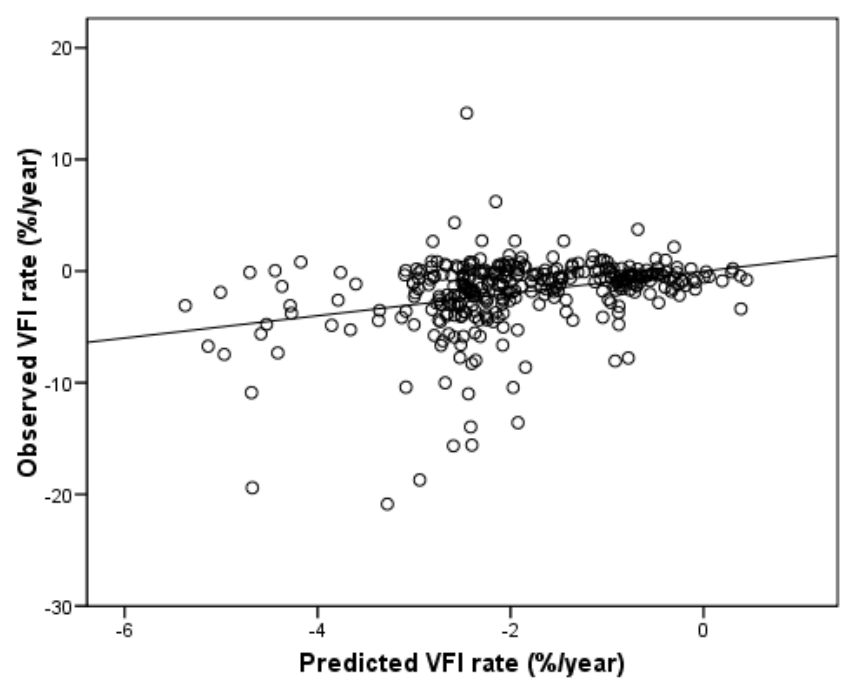

Figure 2a: Calibration plot of the model predicting the rate of progression in the development sample $(n=333)$

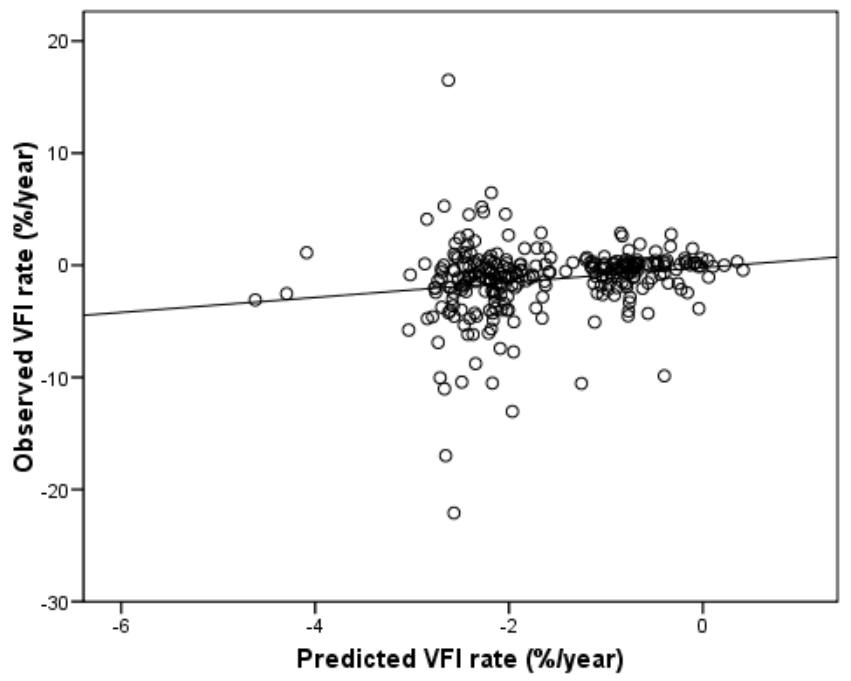

Figure $2 \mathrm{~b}$ : Calibration plot of the internally validated model predicting the rate of progression in the validation sample $(n=280)$

Each point represents the observed VFI rate and the predicted VFI rate of one of the patients included. $\mathrm{VFI}=$ Visual Field Index. 


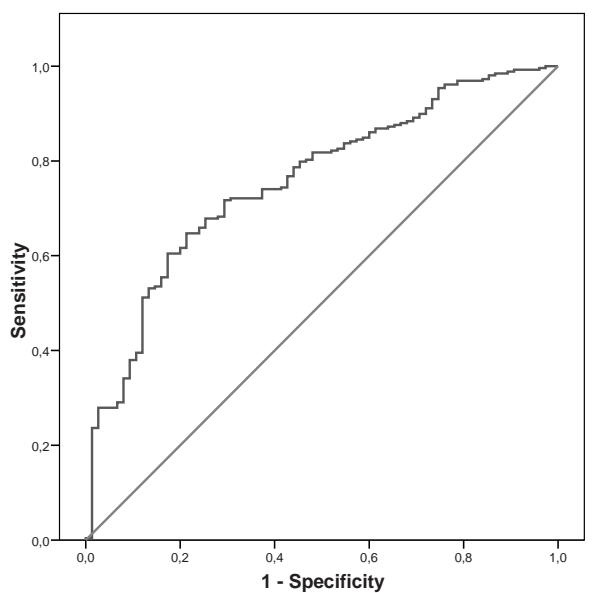

Figure 3: ROC curve for detecting a VFI rate $\leq-3 \%$ per year $(n=333)$

ROC curve $=$ receiver operating characteristic curve, VFI = Visual Field Index.

\section{Model evaluation in the validation sample}

When we applied the internally validated model to individual patients in the validation sample, the mean predicted VFI rate was $-1.6 \%$ (SD \pm 0.9 ) per year. The accompanying calibration plot is shown in Figure 2 b. Fifty out of 280 patients $(17.9 \%)$ in the validation sample had an observed VFI rate $\leq-3 \%$ per year. We found an AUC of 0.71 when we applied the internally validated model to detect a VFI rate $\leq-3 \%$ per year in the validation sample.

When we fitted the model in the validation sample, $R^{2}$ was $5.3 \%$. All coefficients were closer to zero, except for age:

VFI rate $=$

$2.88+-0.05 *$ age $+0.07 *$ IOP $>21 \mathrm{mmHg}+-0.80 *$ moderate MD + -1.06* low MD

The model without IOP had the same $R^{2}$. The coefficients of age and baseline MD were not influenced by the addition of IOP to the model. Therefore, we decided to keep IOP in the model.

\section{Final model}

The final model fitted in the entire population of 613 patients was:

$\mathrm{VFI}$ rate $=$

$2.43+-0.05 *$ age $+-1.52 *$ IOP $>21 \mathrm{mmHg}+-1.20 *$ moderate MD $+-1.52 *$ low MD $R^{2}$ of the final model was $7.6 \%$. 
Table 3: AUCs for detecting different rates of visual field progression ( $n=333)$

\begin{tabular}{lcccc}
\hline Detected VFI rates & Observed cases & Prevalence & AUC & Confidence interval \\
\hline$\leq-1.0 \%$ & 169 & $50.8 \%$ & 0.697 & $0.640,0.753$ \\
$\leq-1.5 \%$ & 138 & $41.4 \%$ & 0.712 & $0.656,0.768$ \\
$\leq-2.0 \%$ & 110 & $33.0 \%$ & 0.743 & $0.688,0.798$ \\
$\leq-2.5 \%$ & 92 & $27.6 \%$ & 0.747 & $0.690,0.804$ \\
$\leq-3.0 \%$ & 75 & $22.5 \%$ & 0.756 & $0.697,0.816$ \\
$\leq-3.5 \%$ & 67 & $20.1 \%$ & 0.755 & $0.696,0.813$ \\
$\leq-4.0 \%$ & 53 & $15.9 \%$ & 0.751 & $0.687,0.816$
\end{tabular}

The number of observed highly progressive cases for each cut-off point with the corresponding prevalence is shown in the second and third columns. The AUC and its confidence interval are shown in the last two columns.

$\mathrm{VFI}=$ Visual Field Index, $\mathrm{AUC}=$ the area under the receiver operating characteristic curve.

Table 4: Accuracy measures for different cut-off points on the ROC curve for detecting a VFI rate $\leq-3 \%$ per year ( $n=333)$

\begin{tabular}{lcccccc}
\hline $\begin{array}{l}\text { Cut-off point of } \\
\text { predicted VFI rate }\end{array}$ & $\begin{array}{c}\text { Predicted } \\
\text { cases }\end{array}$ & $\begin{array}{c}\text { True } \\
\text { positives }\end{array}$ & Sensitivity & Specificity & \multicolumn{2}{c}{ Predictive values } \\
\cline { 4 - 7 }$-1.0 \%$ & 243 & 69 & 0.92 & 0.33 & 0.28 & 0.93 \\
$-1.5 \%$ & 216 & 66 & 0.88 & 0.42 & 0.31 & 0.92 \\
$-2.0 \%$ & 176 & 62 & 0.83 & 0.56 & 0.35 & 0.92 \\
$-2.5 \%$ & 86 & 39 & 0.52 & 0.82 & 0.45 & 0.85 \\
$-3.0 \%$ & 31 & 19 & 0.25 & 0.95 & 0.61 & 0.81 \\
$-3.5 \%$ & 20 & 12 & 0.16 & 0.97 & 0.60 & 0.80 \\
$-4.0 \%$ & 15 & 10 & 0.13 & 0.98 & 0.67 & 0.80 \\
\hline
\end{tabular}

The total number of observed cases with a VFI rate $\leq-3 \%$ per year is 75 , with a prevalence of $22.5 \%$. $\mathrm{ROC}=$ receiver operating characteristic, VFI = Visual Field Index.

\section{Discussion}

Although only a small amount of the variance in visual field progression could be explained by our prediction model, it may offer the possibility to indentify subgroups of treated patients with high rates of glaucomatous visual field progression and provide an opportunity for more intensive treatment of these patients. Three out of eight tested predictors (i.e. age, IOP, and baseline visual field) significantly improved predictions of the VFI rate in the multivariable model. Regression coefficients were consistent at the external validation, with the exception of IOP.

However, we feel that our model should not be used to predict an exact VFI rate for an individual patient, because we found a large scattering in the calibration plot (Figure 2a) 
and a relatively high absolute difference between the observed and predicted VFI rate. The performance of our prediction model improved when it was used to distinguish rapidly progressive and a slowly progressive glaucoma patients. AUCs ranged from 0.743 to 0.756 when detecting VFI rates $\leq-2 \%, \leq-3 \%$, or $\leq-4 \%$ per year (Table 3 ). The accuracy measures of Table 4 reflect the potential clinical value of the prediction model. Without the use of a prediction model, the probability of a VFI rate $\leq-3 \%$ for each individual patient in the development sample is $22.5 \%$ (i.e. the prevalence). With use of our prediction model, this chance becomes $61 \%$ (i.e. the PPV) and 19\% (i.e. 1-NPV) after a positive and negative test result, respectively (Table 4). Hence, a positive result of our prediction model gives a clinical significant change in a patient's risk of future rapid progression.

In conducting the current study, we applied broad selection criteria to give a good representation of patients with OAG in clinical practice. Therefore, we included patients who had at least two reliable visual fields available, although we know that rates of progression based on a low number of visual fields may be less reliable. ${ }^{16}$ We found no significant effect of the number of visual fields performed on the performance of the prediction model $(p=0.410)$. This might be explained by the relatively long follow-up period in this study, resulting in higher signal-to-noise ratios for the rate of progression. ${ }^{17}$ Furthermore, we excluded no patients because of other ocular pathology, like cataract. We used the VFI as outcome measure, which corrects for general visual field depression in patients with early and moderate visual field loss. ${ }^{10}$

In an earlier review, we found 85 studies that investigated prognostic factors for glaucomatous visual field progression in univariate or multivariable models. ${ }^{18}$ However, only a few models have been assessed for their clinical performance in predicting progression. Nouri Mahdavi and co-workers used a prediction model with predictors derived during the first four years of follow-up to predict visual field progression after eight years. ${ }^{19}$ Sensitivity and specificity of the model were actually quite high (i.e. 0.86 and 0.73 respectively) at the best cut-off point for visual field progression in the first four years. ${ }^{19}$ The sensitivity and specificity of our model (i.e. 0.83 and 0.56 respectively, as shown in Table 4) were lower, probably because we used baseline data instead of data collected over a period of four years. In another study, De Moraes and co-workers used an independent population for external validation of their prediction model. ${ }^{20}$ Like in the current study, the $R^{2}$ statistic of their model at external validation (i.e. $14 \%$ ) was low. However, the $c$-index of their model at external validation was 0.78 , which is relatively high compared with the analogous AUC of 0.71 in the current study. This may be partly explained by the inclusion of prognostic factors in their prediction model that were collected during the follow-up period instead of at baseline, like peak IOP and 
mean IOP. ${ }^{20}$ Other studies investigated the performance of models to predict conversion from ocular hypertension to glaucoma. For example, a prediction model for the five-year risk of developing glaucoma in ocular hypertensive patients has been implemented by the Ocular Hypertension Treatment Study (OHTS) Group. ${ }^{21}$ The AUC for this model was 0.76 in the development sample and 0.72 at external validation, which are very comparable with the values of our model. ${ }^{21}$

The three predictors in our final model have shown to be among the most consistent prognostic factors for OAG progression. ${ }^{18,22}$ An elevated IOP is also one of the few factors that can be modified by medical or surgical treatment. ${ }^{23}$ Reducing IOP has been shown to slow the onset and progression of OAG. ${ }^{24,25}$ Therefore, treatment in clinical practice is mainly guided by IOP values. The effect of changing treatment based on IOP could dilute and even reverse the relation between IOP and visual field progression. This may explain why IOP did not contribute to the model in the external validation of our study. Nonetheless, we found a strong effect of baseline IOP with an $R^{2}$ of $3.3 \%$ in the univariate analysis of the development sample. We modeled IOP as a dichotomous variable and found an optimal cut-off point at $21 \mathrm{mmHg}$. This cut-off point matches the traditional border between normal and abnormal IOP values. ${ }^{26}$ Baseline MD was also modeled as a categorical variable, because earlier studies have shown that the relation between visual field progression and baseline visual field loss is not uniform across the entire range of visual field loss. Progression is positively related with baseline visual field severity in early disease stages and negatively related with visual field severity in advanced disease stages. ${ }^{12,27,28}$

Five out of eight tested prognostic factors were not included in the final prediction model because their $p$-value in the univariate pre-selection was $\geq 0.1$. Suboptimal measurement may have contributed to the lack of significance for some of the predictors. For example, adherence to glaucoma medication was defined as whether a patient used eyedrops in the last 24-hours before the baseline visit. There are indications that patient adherence improves close to an upcoming visit. ${ }^{29,30}$ Therefore, adherence in this study may be overestimated. Previous disease progression at baseline was defined as subjective progression according to the treating ophthalmologist. It would be better to measure previous disease progression more objectively by making visual fields in the years before baseline. However, these were not available for most patients in the current study. Not all currently known predictors were investigated in our study because they were not available. For example, we did not study the presence of disc hemorrhages, central corneal thickness (CCT), and peripapillary atrophy. ${ }^{18}$ However, the strength of the current model is the use of simple, easily accessible baseline clinical data. 


\section{References}

1. Kwon YH, Fingert JH, Kuehn MH, Alward WL. Primary open-angle glaucoma. N Engl J Med 2009;360:111324.

2. Resnikoff S, Pascolini D, Etya'ale D, Kocur I, Pararajasegaram R, Pokharel GP, et al. Global data on visual impairment in the year 2002. Bull World Health Organ 2004;82:844-51.

3. Caprioli J. The importance of rates in glaucoma. Am J Ophthalmol 2008;145:191-2.

4. Rossetti L, Goni F, Denis P, Bengtsson B, Martinez A, Heijl A. Focusing on glaucoma progression and the clinical importance of progression rate measurement: a review. Eye 2010;24 Suppl 1:S1-7.

5. Moons KG, Royston P, Vergouwe Y, Grobbee DE, Altman DG. Prognosis and prognostic research: what, why, and how? BMJ 2009;338:b375.

6. Medeiros FA, Weinreb RN. Predictive models to estimate the risk of glaucoma development and progression. Prog Brain Res 2008;173:15-24.

7. van der Valk R, Schouten JS, Webers CA, Hendrikse F, Prins MH. Changes in glaucoma treatment and achieved IOP after introduction of new glaucoma medication. Graefes Arch Clin Exp Ophthalmol 2006;244:1267-72.

8. van der Valk R, Webers CA, Hendrikse F, de Vogel SC, Prins MH, Schouten JS. Predicting intraocular pressure change before initiating therapy: timolol versus latanoprost. Acta Ophthalmol Scand 2008;86:415-8.

9. Artes PH, O'Leary N, Hutchison DM, Heckler L, Sharpe GP, Nicolela MT, et al. Properties of the statpac visual field index. Invest Ophthalmol Vis Sci 2011;52:4030-8.

10. Bengtsson B, Heijl A. A visual field index for calculation of glaucoma rate of progression. Am J Ophthalmol 2008;145:343-53.

11. Werner EB, Drance SM, Schulzer M. Trabeculectomy and the progression of glaucomatous visual field loss. Arch Ophthalmol 1977;95:1374-7.

12. Rao HL, Kumar AU, Babu JG, Senthil S, Garudadri CS. Relationship between Severity of Visual Field Loss at Presentation and Rate of Visual Field Progression in Glaucoma. Ophthalmology 2011;118:249-53.

13. Harrell FE, Jr., Califf RM, Pryor DB, Lee KL, Rosati RA. Evaluating the yield of medical tests. JAMA 1982;247:2543-6.

14. Altman DG, Vergouwe $Y$, Royston $P$, Moons KG. Prognosis and prognostic research: validating a prognostic model. BMJ 2009;338:b605.

15. Steyerberg EW. Clinical prediction models : a practical approach to development, validation, and updating. New York, N.Y.: Springer, 2009.

16. Chauhan BC, Garway Heath DF, Goni FJ, Rossetti L, Bengtsson B, Viswanathan AC, et al. Practical recommendations for measuring rates of visual field change in glaucoma. Br J Ophthalmol 2008;92:56973.

17. Jansonius NM. On the accuracy of measuring rates of visual field change in glaucoma. Br J Ophthalmol 2010;94:1404-5.

18. Ernest PJ, Schouten JS, Beckers HJ, Hendrikse F, Prins MH, Webers CA. An Evidence-Based Review of Prognostic Factors for Glaucomatous Visual Field Progression. Ophthalmology 2013;120:512-9.

19. Nouri Mahdavi K, Hoffman D, Gaasterland D, Caprioli J. Prediction of visual field progression in glaucoma. Invest Ophthalmol Vis Sci 2004;45:4346-51.

20. De Moraes CG, Sehi M, Greenfield DS, Chung YS, Ritch R, Liebmann JM. A validated risk calculator to assess risk and rate of visual field progression in treated glaucoma patients. Invest Ophthalmol Vis Sci 2012;53:2702-7.

21. Gordon MO, Torri V, Miglior S, Beiser JA, Floriani I, Miller JP, et al. Validated prediction model for the development of primary open-angle glaucoma in individuals with ocular hypertension. Ophthalmology 2007;114:10-9. 
22. Coleman AL, Miglior S. Risk factors for glaucoma onset and progression. Surv Ophthalmol 2008;53 Suppl1:S3-10.

23. Bahrami H. Causal inference in primary open angle glaucoma: specific discussion on intraocular pressure. Ophthalmic Epidemiol 2006;13:283-9.

24. Heijl A, Leske MC, Bengtsson B, Hyman L, Bengtsson B, Hussein M. Reduction of intraocular pressure and glaucoma progression: results from the Early Manifest Glaucoma Trial. Arch Ophthalmol 2002;120:126879.

25. Kass MA, Heuer DK, Higginbotham EJ, Johnson CA, Keltner JL, Miller JP, et al. The Ocular Hypertension Treatment Study: a randomized trial determines that topical ocular hypotensive medication delays or prevents the onset of primary open-angle glaucoma. Arch Ophthalmol 2002;120:701-13; discussion 82930.

26. Allingham RR, Shields MB. Shields' textbook of glaucoma, 5th ed, Philadelphia: Lippincott Williams \& Wilkins; 2005.

27. Blackwell B, Gaasterland D, Ederer F, Dally LG, VanVeldhuisen P, Prum BE, et al. The Advanced Glaucoma Intervention Study (AGIS): 12. Baseline risk factors for sustained loss of visual field and visual acuity in patients with advanced glaucoma. Am J Ophthalmol 2002;134:499-512.

28. Leske MC, Heijl A, Hussein M, Bengtsson B, Hyman L, Komaroff E. Factors for glaucoma progression and the effect of treatment: The early manifest glaucoma trial. Arch Ophthalmol 2003;121:48-56.

29. Kass MA, Meltzer DW, Gordon M, Cooper D, Goldberg J. Compliance with topical pilocarpine treatment. Am J Ophthalmol 1986;101:515-23.

30. Okeke CO, Quigley HA, Jampel HD, Ying GS, Plyler RJ, Jiang Y, et al. Adherence with topical glaucoma medication monitored electronically the Travatan Dosing Aid study. Ophthalmology 2009;116:191-9. 
CHAPTER 4

The influence of the assessment method on the incidence of visual field progression in glaucoma: a network meta-analysis

Authors:

Paul J.G. Ernest

Wolfgang Viechtbauer

Jan S.A.G. Schouten

Henny J.M. Beckers

Fred Hendrikse

Martin H. Prins

Carroll A.B. Webers 


\section{ABSTRACT}

Purpose: To study and quantify the difference in incidence of progression between methods for the assessment of glaucomatous visual field progression.

Methods: We identified 2,450 articles published up to April 2009 in the following data sources: PubMed, EMBASE, and Cochrane. Ten studies covering 30 methods were included. All studies aimed to compare different methods for the assessment of glaucomatous visual field progression in the same study population. A network metaanalysis using a mixed-effects model was performed to combine within-study betweenmethod comparisons with indirect comparisons from other studies. The summarized incidence of progression was calculated for every method and methods were ranked according to this incidence.

Results: In total, methods were compared in 1040 eyes of 948 glaucoma patients. On average, $21 \%$ of the eyes progressed. When all 30 methods were ranked, the incidence ranged from $2 \%$ to $62 \%$. These incidences are corrected for a baseline mean deviation (MD) value of -7 decibels and a mean follow-up time of six years. Besides the assessment method, the incidence was only determined by the follow-up period and baseline MD value, leaving no unexplained variance in the incidence of progression.

Conclusion: The incidence of progression varies considerably between different studies. This is mainly caused by the variety of methods used to assess progression, but also by differences in follow-up time and baseline visual field loss. 


\section{Introduction}

Monitoring patients with open-angle glaucoma is based on the reliable detection of early deterioration of the visual field and subsequent lowering of the intraocular pressure. The aim is to prevent irreversible visual field loss leading to visual disability. ${ }^{1} \mathrm{~A}$ major problem in the assessment of glaucomatous visual field progression is the lack of a gold standard. ${ }^{2}$ As a consequence, investigators often develop a new method for their own purposes. Many different methods have been described, ranging from completely subjective to fully automated calculations., 4

If studies are using different methods and subsequently have different results, it is hard to distinguish the contribution of the chosen method from other study characteristics in the quantification of the incidence of progression. This could make studies incomparable to each other. However, the extent to which the use of different methods leads to differences in study outcome between similar studies has not yet been investigated. What has been investigated is the use of different methods in one study population. Most frequently, the mutual agreement of a few methods was investigated by studying the difference in the proportion of patients who develop progression and the overlap in patients classified as having progressed. However, no studies have been published that compare all methods in one study. Therefore, a ranking of methods is missing and it remains unclear which methods give a high or a low incidence of progression. The interpretation and the possibility to compare studies will improve with a clear ranking. For example, it would make a real difference to find a high incidence with a low ranked method when compared to finding a low incidence with a high ranked method.

In the present study, firstly all methods to assess glaucomatous visual field progression used in different studies were compared. Secondly, these methods were ranked according to their incidence of progression and their difference was quantified using a network meta-analytic approach. Finally, we also investigated the influence of baseline visual field loss and follow-up time and determined the extent to which the incidence of progression was explained by these variables.

\section{Materials and methods}

\section{Search strategy}

A systematic computerized search was performed in PubMed, EMBASE, and all databases and registers of The Cochrane Library, in April 2009. The following keywords were used: (glaucoma*) AND (prognos* OR predict* OR progress* OR longitudinal OR cohort 
OR follow-up) AND (perimetr* OR visual field* OR HFA OR Octopus OR Humphrey (not in author)). The search was limited to articles in English, Dutch, French, or German. A total of 2,450 articles were identified.

\section{Selection procedure}

All titles and abstracts were screened and articles were excluded based on predefined exclusion criteria. Of the remaining 782 articles that were studied completely, 48 articles fulfilled the selection criteria (figure 1). The selected studies had to follow glaucoma patients for minimally one year with the use of conventional visual field examinations. Studies should also directly compare at least two methods to assess glaucomatous visual field progression within the same study population. After that, the type of perimeter used and a precise description of the methods used for assessment of visual field progression were recorded for these studies. In addition, the number of participants, duration of follow-up, number of visual field examinations, patient characteristics, incidence of progression, and the publication year were noted.

We combined direct and indirect comparisons of methods in a network meta-analysis. A direct comparison is defined as a comparison of methods in one study using exactly the same study population during the same study period. Indirect comparisons of two methods have not actually been made in one study, but are made in different studies by using a common comparator. ${ }^{5}$ Studies were excluded when no comparisons with other methods in the meta-analysis were possible. This led to the sole inclusion of studies that applied methods based on the Humphrey Field Analyzer (HFA) (Carl Zeiss Meditec, Dublin, CA, USA), since this perimeter was used in most studies on progression and no studies were found that directly compared HFA-based methods with methods from other perimeters. Furthermore, studies that did not report incidence proportions of progression and in which the incidence proportion could not be calculated from the data were excluded. We defined the incidence proportion as the proportion of glaucoma patients that developed visual field progression during the follow-up period of a specific study. Studies with populations that overlapped with other selected studies were also excluded to obtain a fair comparison between methods. In these cases, the study was chosen that had the most comparisons with methods used in other studies. When overlap of study populations was uncertain, more information was retrieved from the authors. Finally, two studies were found which investigated a selected group of glaucoma patients that was considered to be progressive based on clinical judgement. ${ }^{6}$

${ }^{7}$ These studies were excluded, because including them would result in selection bias. ${ }^{8}$ 
2450 potential relevant articles identified and screened for retrieval

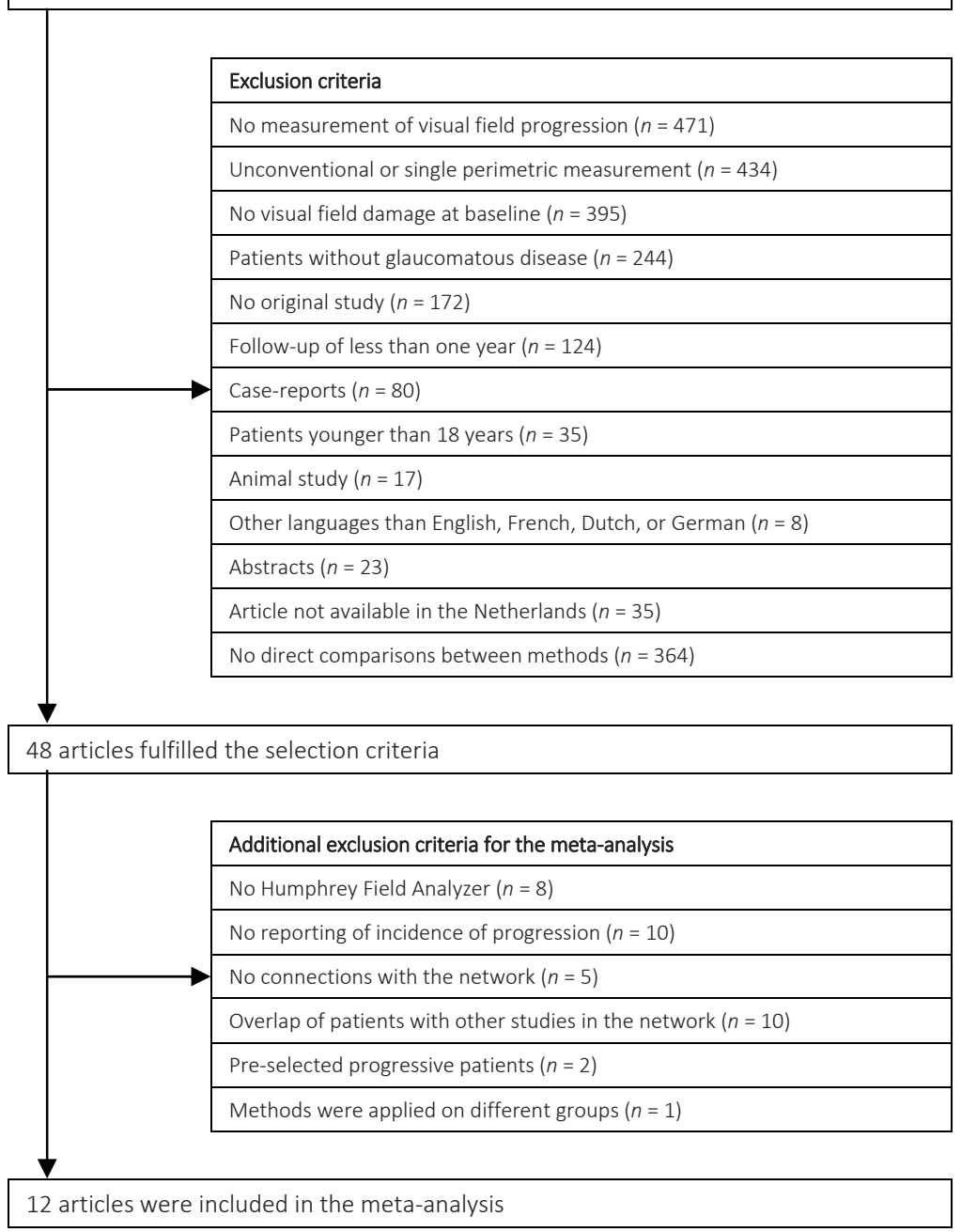

Figure 1: Flow chart of the article selection process

Finally, the data of twelve articles were combined in the network meta-analysis (figure 1). ${ }^{9-20}$ Three articles were included that studied different methods in the same population during the same follow-up period. ${ }^{12-14}$ Data of these articles were treated as a single study. The references of all included articles were additionally screened to identify more studies. This did not result in additional articles. However, we found one recent article that described the same study as one of the included articles. ${ }^{21}$ Since additional methods were applied in this article, we added these methods to the meta-analysis. 


\section{Statistical analysis}

If not available, incidence proportions of glaucomatous progression were calculated from the number of eyes that progressed for each method within each study. All incidence proportions were then combined in a mixed-effects model. More specifically, an arm-based network meta-analysis model with dummy variables to identify the various methods was used for the analysis. ${ }^{22}$ The computations for these analyses were performed with the statistical software R (version 2.10.1). An additional complication in the analysis arose due to the fact that incidence proportions from the same sample using different methods are correlated and therefore cannot be treated as independent observations. The magnitude of the correlation between several incidence proportions from the same sample can only be calculated if a complete cross-classification table of the various methods is provided in an article. Usually, such detailed information was unavailable. We therefore used the method suggested by Riley and co-workers. ${ }^{23}$ This method substitutes a single overall correlation parameter for the study-specific individual correlations.

The model therefore is given by:

$$
y_{i j}=B_{1} M_{1, i j}+b_{2} M_{2, i j}+\ldots+b_{x} M_{x, i j}+u_{i j}+\varepsilon_{i j}
$$

where $y_{i j}$ is the observed incidence proportion in study $i$ for method $j, M_{1, i j}$ is a dummy variable (i.e., coded 1 or 0 ), indicating whether $y_{i j}$ corresponds to method 1 (and similarly for $M_{2, \mathrm{ij}}$ through $M_{\mathrm{x}, \mathrm{ij}}$ ), the $u_{\mathrm{ij}}$ values denote the random effects, and the $\varepsilon_{\mathrm{ij}}$ values denote the sampling errors. The model assumes that the variances of the $u_{i j}$ 's are equal to $\tau^{2}$, denoting the amount of heterogeneity in the true incidence proportions. Furthermore, the correlation between two $u_{i j}$ 's from the same sample (i.e., study) is denoted by $\rho$. The variances of the $\varepsilon_{\mathrm{ij}}$ values were estimated based on the observed incidence proportions and can be denoted by $v_{\mathrm{ij}}$. Moreover, two $\varepsilon_{\mathrm{ij}}$ values from the same study are also assumed to be related to each other with their correlation given by $\rho$. Finally, assuming normality of the $u_{\mathrm{ij}}$ and $\varepsilon_{\mathrm{ij}}$ values, the parameters of the model were then estimated using restricted maximum likelihood estimation. As a result, we obtained the following estimates from the model: the average incidence proportions for the various methods (estimated values of $b_{1}$ through $B_{x}$ ), the amount of heterogeneity in these results (estimate of $\tau^{2}$ ), and an estimate of the correlation for incidence proportions arising from the same study (estimate of $\rho$ ). The precision of the estimates of $B_{1}$ through $B_{x}$ was quantified via corresponding $95 \%$ confidence intervals obtained in the usual manner (i.e., estimate of $B \pm 1.96 \mathrm{SE}$, where SE denotes the standard error of the estimate of $B$ ). A confidence interval was also obtained for $\tau^{2}$, based on a profile likelihood method. ${ }^{24}$ 
The mean deviation (MD) value at baseline and the mean follow-up time were also included as covariates in the model so that the incidence proportions from the various methods could be compared while controlling for the influence of these covariates. We included these covariates initially as linear predictors in the model, but also examined whether the incidence proportions changed as an accelerating (quadratic) function thereof. Another potential covariate was the mean number of visual field examinations for the patients in each study. However, this covariate correlated very strongly with the mean follow-up time in a study $(r=0.79)$ and therefore it was almost impossible to distinguish. We chose to use follow-up time over the number of examinations because its influence on the incidence of progression is inherently more logical.

Where possible, we also compared the incidence proportions of two methods from the network model with incidence proportions of the same two methods, which were based only on direct comparisons. To use a reasonable amount of data for getting more stable results, this additional analysis was only done for methods that were directly compared in three or more studies.

\section{Results}

The flow of articles included in this systematic literature review is shown in figure 1. Twelve articles that studied 30 methods in ten studies were included in the metaanalysis. All methods were named and classified in six groups according to their main characteristics (table 1).

\section{Six groups of methods}

The first group consisted of methods based on the Glaucoma Progression Analysis (GPA), which is similar to the visual field endpoint in the Early Manifest Glaucoma Trial (EMGT). ${ }^{25}$ We abbreviated this group to "group G", from GPA. The GPA is an event analysis based on pattern deviation values and is included in the new HFA software. When significant deterioration $(p<0.05)$ is seen on the pattern deviation probability maps of the GPA printouts in the same three or more points on three consecutive follow-up tests, the software interprets this as likely progression. ${ }^{25}$ Although some studies performed separate analyses after exclusion of eyes with a baseline visual field "out of range" according to the GPA printout of the HFA, we used the incidences of progression in all study eyes. Group A (A from AGIS) consisted of two methods that were based on the Advanced Glaucoma Intervention Study (AGIS). ${ }^{26}$ Both the AGIS method and the Collaborative Initial Glaucoma Treatment Study (CIGTS) method, which is derived from the AGIS method, use a scoring system to grade each visual field in the 
follow-up period. The AGIS score is based on the actual decibel deviations at the total deviation plot, while the CIGTS algorithm is based on the p-values obtained from the total deviation probability plot. Both scoring systems range from 0 to 20, with 0 representing no field loss and 20 end-stage disease. Visual field series are considered to be progressive if the score has a minimal increase of four (with the AGIS method) or three points (with the CIGTS method) and is confirmed by two additional tests. ${ }^{27}$ In methods that are classified as point-wise linear regression (PLR) methods (group P from PLR), a linear regression analysis was performed in different individual locations at the visual field. Another group of methods was based on a linear regression analysis with visual field indices (group I from indices), like the MD and the Visual field index (VFI). The VFI is calculated by the new software of the HFA. Each location on the visual field contributes to the VFI, although it is more heavily weighted to central areas of the visual field. ${ }^{28} \mathrm{~A}$ location which is not significantly $(p<0.05)$ depressed on the pattern deviation probability map is considered to have a $100 \%$ sensitivity. The VFI is expressed as one percentage, where $100 \%$ represents a normal visual field and $0 \%$ represents a perimetrically blind eye. The HFA software performs a linear regression analysis of the VFI against time. $^{28}$

Another group of methods was made that combined a PLR and a linear regression analysis of the MD value. The methods in this group (group T from TNT) were variants of the Threshold Noiseless Trend (TNT) program. ${ }^{29}$ The TNT program filters perimetric results and takes into account dependency relations in the visual field. Moreover, it combines linear regression analyses of the MD, the cumulative defect curve, and different locations at the visual field. Suspected progression is seen the first time that one of these parameters indicates progression. If this result is repeated by two consecutive examinations or if two or more parameters indicate progression, TNT indicates definite progression. ${ }^{17}$ Finally, a clinical group (group C from clinical) comprised methods based on clinical judgement. Classified in this group were methods based on entirely subjective assessments of visual fields by multiple observers, who had to agree on progression while they were blinded for other clinical data. Other methods in this group used certain algorithms for the assessment of visual fields, for example based on the clinical judgement of scotoma's. ${ }^{30}$ One clinical method was based on nonparametric ranking of MD values. ${ }^{19}$ This method objectifies the commonly practised method of monitoring glaucoma patients with the use of MD values. A visual field series is considered progressive as the MD value of a follow-up visual field is worse than the MD of the worse of two baseline fields. This has to be confirmed on at least two visual fields. ${ }^{19}$ We did not take into account one subjective method that was based on the experience and expertise of only one observer who was not blinded for other clinical information. ${ }^{11}$ 
Table 1: Description of the 30 methods studied

\begin{tabular}{|c|c|}
\hline G & Based on Glaucoma Progression Analysis (GPA) \\
\hline G1 & GPA \\
\hline G2 & GPA using total deviation \\
\hline G3 & GPA with 2 follow-up visual fields \\
\hline G4 & $\begin{array}{l}\text { Modified GPA; difference between number of improving points and deteriorating points }(\geq 3) \text { repeatable at } 3 \\
\text { consecutive examinations }\end{array}$ \\
\hline G5 & $\begin{array}{l}\text { Modified GPA using total deviation; difference between number of improving points and deteriorating points }(\geq 3) \\
\text { repeatable at } 3 \text { consecutive examinations }\end{array}$ \\
\hline A & Based on Advanced Glaucoma Intervention Study (AGIS) method \\
\hline A1 & AGIS-method \\
\hline A2 & CIGTS-method \\
\hline $\mathbf{P}$ & Based on point-wise linear regression analysis (PLR) \\
\hline$P 1$ & PLR with threshold values, using a criterion of $p \leq 0.001$ and a cut-off point of $\geq 4$ locations \\
\hline $\mathrm{P} 2$ & PLR with threshold values, using a criterion of $p \leq 0.001$ and a cut-off point of $\geq 3$ locations \\
\hline P3 & PLR with threshold values, using a criterion of $p \leq 0.001$ and a cut-off point of $\geq 2$ locations \\
\hline P4 & PLR with threshold values, using a criterion of $p \leq 0.001$ and a cut-off point of $\geq 1$ location \\
\hline P5 & $\begin{array}{l}\text { Two-omitting PLR with pattern deviation values, using criteria of } \leq-1 \mathrm{~dB} \text { per year and } p<0.05 \text { and a cut-off point of } \geq 3 \\
\text { locations at } 3 \text { consecutive examinations }\end{array}$ \\
\hline P6 & $\begin{array}{l}\text { Two-omitting PLR with total deviation values, using criteria of } \leq-1 \mathrm{~dB} \text { per year and } \mathrm{p}<0.05 \text { and a cut-off point of } \geq 3 \\
\text { locations at } 3 \text { consecutive examinations }\end{array}$ \\
\hline P7 & $\begin{array}{l}\text { PLR with total deviation values, using criteria of } \leq-1 \mathrm{~dB} \text { per year and } p \leq 0.01 \text { and a cut-off point of } \geq 3 \text { locations at } 3 \\
\text { consecutive examinations, with consistent worsening through the end of follow-up }\end{array}$ \\
\hline P8 & $\begin{array}{l}\text { PLR with PROGRESSOR software (threshold values), using criteria of } \leq-1 \mathrm{~dB} \text { per year and } p \leq 0.05 \text { and a cut-off point } \\
\text { of } \geq 2 \text { nonedge locations }\end{array}$ \\
\hline $\mathbf{I}$ & Based on linear regression analysis with indices \\
\hline 11 & $\begin{array}{l}\text { Linear regression analysis of a Glaucoma Hemifield cluster, using a criterion of } p<0.005 \text { and a cut-off point of } \geq 4 \\
\text { clusters }\end{array}$ \\
\hline 12 & $\begin{array}{l}\text { Linear regression analysis of a Glaucoma Hemifield cluster, using a criterion of } p<0.005 \text { and a cut-off point of } \geq 3 \\
\text { clusters }\end{array}$ \\
\hline 13 & $\begin{array}{l}\text { Linear regression analysis of a Glaucoma Hemifield cluster, using a criterion of } p<0.005 \text { and a cut-off point of } \geq 2 \\
\text { clusters }\end{array}$ \\
\hline 14 & $\begin{array}{l}\text { Linear regression analysis of a Glaucoma Hemifield cluster, using a criterion of } p<0.005 \text { and a cut-off point of } \geq 1 \\
\text { cluster }\end{array}$ \\
\hline 15 & Linear regression analysis of the MD, using a criterion of $p<0.025$ \\
\hline 16 & Linear regression analysis of the MD, using a criterion of $p<0.05$ \\
\hline 17 & Linear regression analysis of the CPSD, using a criterion of $p<0.025$ \\
\hline 18 & Linear regression analysis of the $\mathrm{VFI}$, using a criterion of $\mathrm{p}<0.05$ \\
\hline $\mathbf{T}$ & Based on PLR and linear regression analysis with indices \\
\hline T1 & Progression according to Threshold Noiseless Trend (TNT) program \\
\hline $\mathrm{T} 2$ & Suspected progression according to TNT program \\
\hline $\mathbf{C}$ & Based on clinical judgement \\
\hline $\mathrm{C} 1$ & $\begin{array}{l}\text { Likely progression based on the subjective assessment of Humphrey printouts by multiple observers without using a } \\
\text { predefined criterion; observers should reach agreement and were blinded for other clinical information }\end{array}$ \\
\hline $\mathrm{C} 2$ & $\begin{array}{l}\text { Likely progression based on the subjective assessment of visual fields by multiple observers using PROGRESSOR } \\
\text { software without a predefined criterion; observers should reach agreement and were blinded for other clinical } \\
\text { information }\end{array}$ \\
\hline $\mathrm{C} 3$ & Nonparametric progression analysis based on nonparametric ranking of MD values \\
\hline $\mathrm{C} 4$ & $\begin{array}{l}\text { At least one of these scotoma criteria: deepening of } \geq 2 \text { points } \geq 10 \mathrm{~dB} \text { in the same locations; expansion of } \geq 2 \text { points } \\
\geq 10 \mathrm{~dB} \text {; development of } \geq 2 \text { adjacent points, not within or adjacent to a baseline scotoma, with } p<1 \% \text { or worse at } \\
\text { pattern deviation plot or } 1 \text { previously normal location in central } 10 \text { degrees } \geq 10 \mathrm{~dB} \text {. Confirmations at the same } \\
\text { locations on } 3 \text { consecutive fields, based on pattern deviation values }\end{array}$ \\
\hline C5 & $\begin{array}{l}\text { At least one of these scotoma criteria: deepening of } \geq 2 \text { points } \geq 10 \mathrm{~dB} \text { in the same locations; expansion of } \geq 2 \text { points } \\
\geq 10 \mathrm{~dB} \text {; development of } \geq 2 \text { adjacent points, not within or adjacent to a baseline scotoma, with } p<1 \% \text { or worse at } \\
\text { pattern deviation plot or } 1 \text { previously normal location in central } 10 \text { degrees } \geq 10 \mathrm{~dB} \text {. Confirmations at the same } \\
\text { locations on } 2 \text { consecutive fields, based on pattern deviation values }\end{array}$ \\
\hline
\end{tabular}

Methods are classified in six groups according to their main characteristics.

GPA = Glaucoma Progression Analysis, AGIS = Advanced Glaucoma Intervention Study, CIGTS = Collaborative Initial Glaucoma Treatment Study, PLR = point-wise linear regression, $\mathrm{dB}=$ decibels, $\mathrm{MD}=$ mean deviation, CPSD = corrected pattern standard deviation, VFI = Visual Field Index, TNT = Threshold Noiseless Trend 


\section{Population characteristics}

In total, 1040 eyes of 948 glaucoma patients were studied in the ten studies included. As shown in table 2, all patients were derived from western countries, with mean baseline MD values ranging from -3.3 to -10.4 decibel $(\mathrm{dB})$, and mean age ranging from 57.7 to 72.5 years. An average of 1.7 visual fields per year were analysed in the studies. In one study, patients received laser therapy or filtration surgery according to a treatment protocol. ${ }^{15}$ All other patients received various treatments during the follow-up period. An average of six methods was directly compared in the studies included. The uncorrected incidence proportions that were given or calculated for the different methods in the studies are also shown in table 2. The maximum difference in the incidence proportions within a single study was 0.44 .

\section{Ranking of methods}

Figure 2 shows the ranking of the 30 methods according to their incidence proportions when combined in the meta-analysis. The mean estimated incidence proportion of progression was 0.21 (95\% confidence interval $(\mathrm{Cl}) 0.15,0.26)$ in 6 years (dashed vertical line in figure 2), indicating that on average $21 \%$ of the study eyes progressed in six years. The incidence proportions of progression according to 30 methods ranged from $0.02(\mathrm{Cl}-0.02,0.05)$ for method P1 to $0.62(\mathrm{Cl} 0.47,0.78)$ for method P8. A continuous vertical line represents the incidence proportion of the GPA (method G1), which was the most frequently studied method, with six studies in this meta-analysis. With an incidence proportion of $0.16(\mathrm{Cl} 0.14,0.19)$, the GPA is in the middle of the ranking of all 30 methods. The AGIS-based methods and most of the methods based on linear regression analysis with indices showed lower incidence proportions than the GPA method. Methods that are based on clinical judgement or the TNT program showed higher incidences than the GPA. All incidence proportions shown in figure 2 are the estimated rates when the mean follow-up time was set at six years and the mean baseline MD value was set at $-7 \mathrm{~dB}$. These values were chosen because these were the mean values in the ten included studies. Follow-up time was significantly associated with the incidence of progression, with an increase in the incidence proportion of approximately 2.1 percent points per extra follow-up year $(p<0.001)$. Baseline MD was also significantly associated with the incidence of progression, increasing the incidence proportion by 0.9 percent points per extra dB of MD loss $(p=0.025)$. No accelerating (quadratic) relationships between these two predictors and the incidence proportions were found $(p=0.93$ and $\mathrm{p}=0.77$, respectively). 
Table 2: Characteristics of the ten studies included

\begin{tabular}{|c|c|c|c|c|c|c|c|c|c|c|c|}
\hline $\begin{array}{c}\text { Study } \\
\text { (year of } \\
\text { publication) }\end{array}$ & $\begin{array}{l}\text { Source of the } \\
\text { study population }\end{array}$ & Method & $\begin{array}{l}\text { Uncorrected } \\
\text { incidence } \\
\text { proportion of } \\
\text { progression }\end{array}$ & $\begin{array}{l}\text { Maximum } \\
\text { difference in } \\
\text { incidence of } \\
\text { progression }\end{array}$ & $\begin{array}{r}\text { Num } \\
0 \\
\text { patie } \\
\text { and }\end{array}$ & $\begin{array}{l}\text { nber } \\
\text { f } \\
\text { ents } \\
\text { eyes }\end{array}$ & $\begin{array}{c}\text { Mean } \\
\text { baseline } \\
\text { MD value } \\
\text { (dB) }\end{array}$ & $\begin{array}{l}\text { Mean } \\
\text { age } \\
\text { (years) }\end{array}$ & $\begin{array}{l}\text { Mean } \\
\text { follow-up } \\
\text { period } \\
\text { (years) }\end{array}$ & $\begin{array}{c}\text { Mean } \\
\text { number of } \\
\text { visual field } \\
\text { examin- } \\
\text { ations }\end{array}$ & $\begin{array}{c}\text { Number of } \\
\text { visual } \\
\text { fields per } \\
\text { year }\end{array}$ \\
\hline \multirow{2}{*}{$\begin{array}{c}\text { Birch et al. } \\
\text { (1995) }\end{array}$} & Royal Liverpool & P8 & 0.56 & \multirow[t]{2}{*}{0.42} & \multirow[t]{2}{*}{ NA } & \multirow[t]{2}{*}{36} & \multirow[t]{2}{*}{-7.5} & \multirow[t]{2}{*}{ NA } & \multirow[t]{2}{*}{$3.3^{*}$} & \multirow[t]{2}{*}{6} & \multirow[t]{2}{*}{1.8} \\
\hline & $\begin{array}{l}\text { University } \\
\text { Hospital, UK }\end{array}$ & 16 & 0.14 & & & & & & & & \\
\hline \multirow{10}{*}{$\begin{array}{l}\text { Smith et al. } \\
\text { (1996) }\end{array}$} & \multirow{10}{*}{$\begin{array}{l}\text { Glaucoma } \\
\text { Service, Wilmer } \\
\text { Institute, } \\
\text { Baltimore, } \\
\text { Maryland, USA }\end{array}$} & P1 & 0.05 & \multirow[t]{10}{*}{0.44} & \multirow[t]{10}{*}{191} & \multirow[t]{10}{*}{191} & \multirow[t]{10}{*}{-8.7} & \multirow[t]{10}{*}{61.8} & \multirow[t]{10}{*}{7.1} & \multirow[t]{10}{*}{9.5} & \multirow[t]{10}{*}{1.3} \\
\hline & & P2 & 0.07 & & & & & & & & \\
\hline & & P3 & 0.11 & & & & & & & & \\
\hline & & P4 & 0.19 & & & & & & & & \\
\hline & & 11 & 0.06 & & & & & & & & \\
\hline & & 12 & 0.07 & & & & & & & & \\
\hline & & 13 & 0.09 & & & & & & & & \\
\hline & & 14 & 0.18 & & & & & & & & \\
\hline & & 15 & 0.49 & & & & & & & & \\
\hline & & 17 & 0.14 & & & & & & & & \\
\hline \multirow{14}{*}{$\begin{array}{c}\text { Katz et al. } \\
(1997,1999, \\
2000)\end{array}$} & \multirow{14}{*}{$\begin{array}{c}\text { Glaucoma } \\
\text { Screening Study, } \\
\text { Baltimore, } \\
\text { Maryland, USA }\end{array}$} & G1 & 0.21 & \multirow[t]{14}{*}{0.30} & 56 & 67 & -7.4 & 62.0 & $6.3^{* *}$ & $7^{* *}$ & 1.1 \\
\hline & & $\mathrm{G} 2$ & 0.33 & & & & & & & & \\
\hline & & $\mathrm{A} 1$ & 0.10 & & & & & & & & \\
\hline & & $\mathrm{A} 2$ & 0.19 & & & & & & & & \\
\hline & & P2 & 0.03 & & & & & & & & \\
\hline & & P3 & 0.10 & & & & & & & & \\
\hline & & P4 & 0.22 & & & & & & & & \\
\hline & & 11 & 0.06 & & & & & & & & \\
\hline & & 12 & 0.10 & & & & & & & & \\
\hline & & 13 & 0.12 & & & & & & & & \\
\hline & & 14 & 0.25 & & & & & & & & \\
\hline & & 15 & 0.18 & & & & & & & & \\
\hline & & 17 & 0.07 & & & & & & & & \\
\hline & & C1 & 0.21 & & & & & & & & \\
\hline Lee et al. & DIGS, University & G1 & 0.08 & 0.09 & 48 & 48 & -4.7 & 57.7 & 2.8 & 5 & 1.8 \\
\hline (2002) & of California, San & $\mathrm{G} 2$ & 0.13 & & & & & & & & \\
\hline & Diego (and other & G4 & 0.08 & & & & & & & & \\
\hline & & G5 & 0.13 & & & & & & & & \\
\hline & & $\mathrm{A} 1$ & 0.04 & & & & & & & & \\
\hline & & A2 & 0.04 & & & & & & & & \\
\hline Viswanathan & Moorfields Eye & $\mathrm{C} 1$ & 0.19 & 0.22 & 27 & 27 & $-7.7 * *$ & $61^{* *}$ & $5.7^{* *}$ & 16 & 2.8 \\
\hline et al. (2003) & $\begin{array}{c}\text { Hospital, London, } \\
\text { UK }\end{array}$ & C2 & 0.48 & & & & & & & & \\
\hline Artes et al. & QEII Health & G1 & 0.21 & 0.15 & 101 & 168 & $-3.3^{* *}$ & $61^{* *}$ & $10^{* *}$ & $21^{* *}$ & 2.1 \\
\hline (2005) & Sciences Centre, & G2 & 0.30 & & & & & & & & \\
\hline & Halifax, Nova & P5 & 0.24 & & & & & & & & \\
\hline & Scotia, Canada & P6 & 0.36 & & & & & & & & \\
\hline Nouri-Mahdavi & Advanced & $\mathrm{G} 2$ & 0.31 & 0.13 & 156 & 156 & -10.4 & 64.4 & 8 & $18^{* *}$ & 2.3 \\
\hline et al. (2007) & Glaucoma & $\mathrm{A} 1$ & 0.22 & & & & & & & & \\
\hline & $\begin{array}{l}\text { Intervention } \\
\text { Study, USA }\end{array}$ & P7 & 0.35 & & & & & & & & \\
\hline
\end{tabular}


Table 2: continued

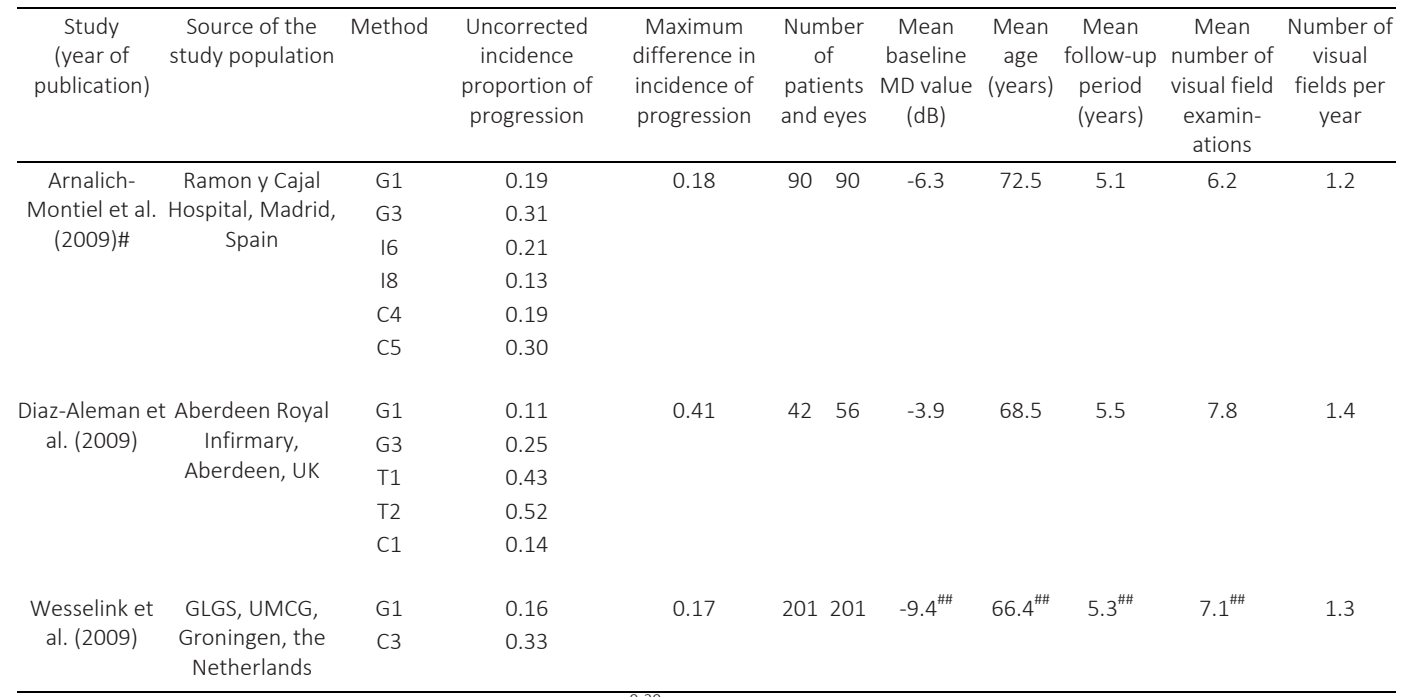

All data were directly derived from the articles, ${ }^{9-20}$ but we calculated the number of visual fields per year by dividing the number of visual field examinations by the follow-up period.

$*$ = the follow-up period for the total group of glaucoma patients and patients with ocular hypertension was used

** = median value

\# = data were derived from a more recent article, ${ }^{21}$ which described the same study

$\# \#=$ data for the total group of 221 patients was used.

$\mathrm{MD}=$ mean deviation, $\mathrm{dB}=$ decibels, $\mathrm{NA}=$ data not available, DIGS = Diagnostic Innovations in Glaucoma Study, GLGS = Groningen Longitudinal Glaucoma Study, UMCG = University Medical Center Groningen. 


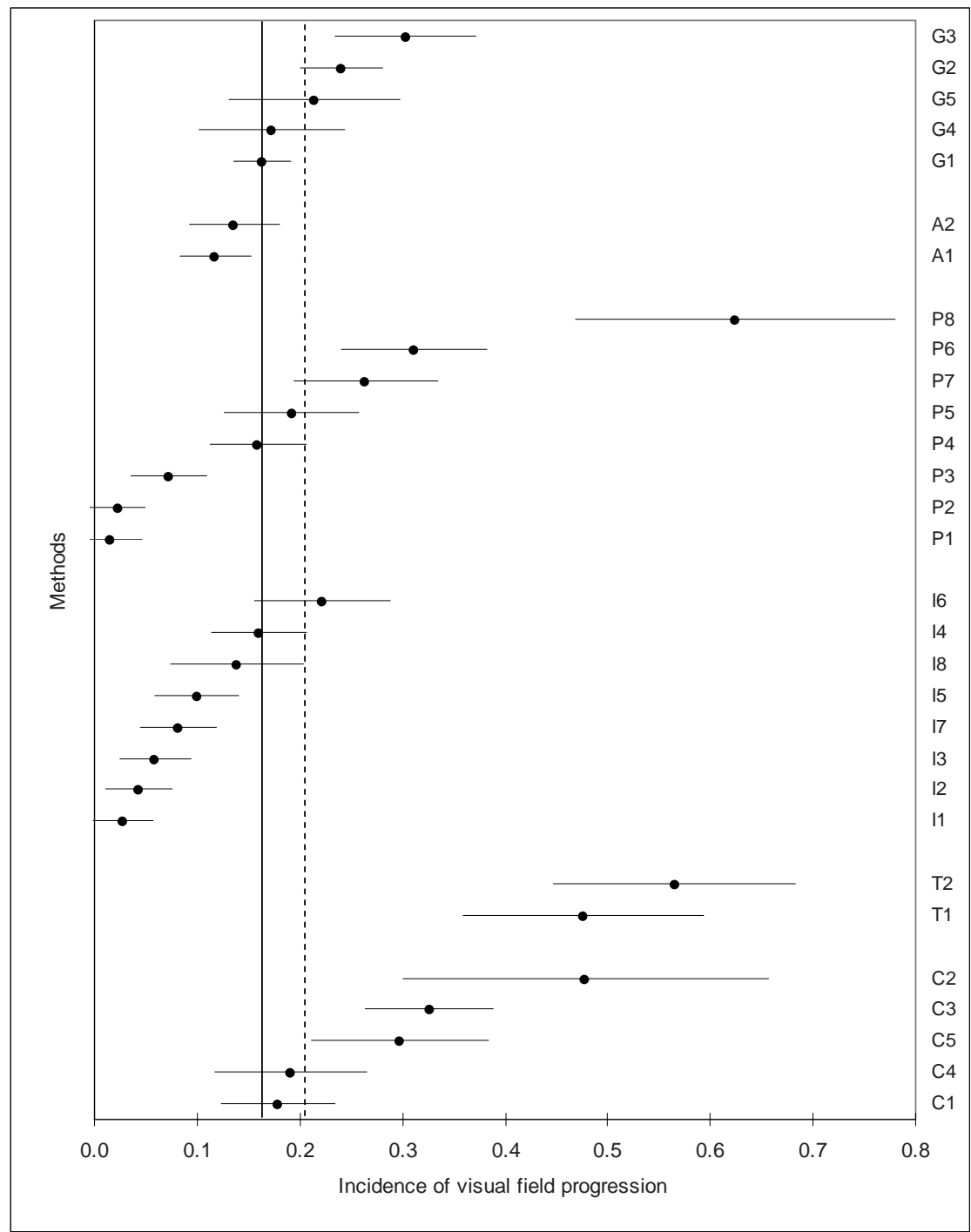

Figure 2: Ranking of 30 methods according to their incidence of visual field progression

The 30 points represent the estimated incidences of visual field progression with their $95 \%$ confidence intervals of all methods studied. The incidences are corrected for a mean follow-up period of six years and a baseline mean deviation value of -7 decibels. The dashed vertical line represents the mean estimated incidence of visual field progression in the ten studies. The continuous vertical line represents the incidence of the Glaucoma Progression Analysis (method G1). The methods are grouped according to their main characteristics: group $G=$ methods based on Glaucoma Progression Analysis, group A = methods based on Advanced Glaucoma Intervention Study method, group $\mathrm{P}=$ methods based on point-wise linear regression analysis, group $\mathrm{I}=$ methods based on linear regression analysis with indices, group $\mathrm{T}=$ methods based on point-wise linear regression analysis and linear regression analysis with indices, group $\mathrm{C}=$ methods based on clinical judgement. 


\section{Contribution of methods}

The variance explained by the model can be expressed in terms of the amount of heterogeneity as estimated by the model (i.e., the estimate of $\tau^{2}$ ). After correcting for follow-up time and baseline MD, the methods used for assessing progression accounted for all the remaining heterogeneity between the estimated incidences of progression in studies $(\mathrm{Cl}$ 0, 0.002). To estimate the contribution of the chosen method in the quantification of the incidence of progression, the model in which all methods were assumed to have the same average incidence of progression (without including mean follow-up time and MD value as covariates) was compared with the model where all methods were allowed to have different incidences. The former model yielded an estimated amount of heterogeneity (i.e., the estimate of $\tau^{2}$ ) equal to $0.011(\mathrm{Cl} 0.006,0.022)$, which is decreased to $0.002(\mathrm{Cl}<0.001,0.008)$ when all methods were allowed to have different incidences. Therefore, approximately $82 \%$ of the heterogeneity in this analysis can be accounted for by the variety of methods used in studies. The rest of the heterogeneity was explained by the mean baseline MD value and the mean follow-up time.

\section{Additional analysis}

We evaluated the network model with both direct and indirect comparisons of methods by performing an additional analysis for the direct comparisons only. This analysis was also corrected for the mean follow-up time and the mean baseline MD. Methods G1 and G2 were the only methods that were directly compared in three or more studies. The estimated difference in the incidence proportion between methods $\mathrm{G} 1$ and $\mathrm{G} 2$ was $0.081(\mathrm{Cl} 0.043,0.120)$ in the model with only direct comparisons, while the difference in incidence was $0.077(\mathrm{Cl} 0.033,0.121)$ in the combined direct and indirect model. The direction and size of the difference between methods $\mathrm{G} 1$ and $\mathrm{G} 2$ was approximately the same in the direct model as in the combined direct and indirect model, although the combined model had a broader confidence interval.

\section{Discussion}

The mean incidence of glaucomatous visual field progression that we found in the literature was $21 \%$ in six years. This incidence was based on a large number of patients and a large number of methods to assess progression and can be seen as an average proportion of eyes that were considered to be progressive. The incidence of visual field progression had a large range from $2 \%$ to $62 \%$ in six years, depending on the method that was used. This again confirms the importance of the choice of a method to assess glaucomatous visual field progression. We then ranked these methods according to their incidence of progression. It is important to note that the incidences that we have 
found for each method can only be generalized for clinically treated glaucoma patients with a mean baseline MD value around $-7 \mathrm{~dB}$ and a mean follow-up time of six years. The estimates of progression should be corrected by adding $0.9 \%$ to the incidence or by subtracting $0.9 \%$ from the incidence, for each $\mathrm{dB}$ decrease and increase in baseline MD value respectively. In the same way, the incidences should be corrected by adding $2.1 \%$ for each year extra follow-up. The expected incidence of progression in an article that used another method can thus be estimated with the ranking presented in this article.

Together with the range of incidences of progression, we also investigated to what extent the use of different methods will lead to differences in results between studies. The chosen method accounted for nearly all differences in the incidence of progression that we found in the included studies, with the exception of the part that can be explained by the baseline MD value and the follow-up time. This underscores the importance of taking account of the method used when critically appraising and comparing studies on glaucomatous visual field progression. However, the present study also underlines the influence of baseline visual field loss and follow-up time on the incidence of glaucomatous visual field progression. When clinicians use the same method, these factors are even more important in clinical practice than the method that is used. Although a longer follow-up period logically leads to an increase in the incidence of visual field progression, a worse baseline visual field increases the chance of future visual field progression in an individual patient. It has been found in previous studies that more advanced baseline visual field loss was an independent predictor of future visual field progression. ${ }^{31}$ However, other studies have found a nonlinear relation of baseline visual field and subsequent progression ${ }^{32,33}$ or even an opposite relation. ${ }^{34}$ The latter finding could be explained by floor effects due to advanced visual field loss of the AGIS patients at baseline. This means that no worsening can be observed when visual field loss is already advanced, but instead even an improvement of the visual field that is most likely the result of regression to the mean. With a mean baseline MD value of $-7 \mathrm{~dB}$, we assume that floor effects had no major influence on the present results.

Although most meta-analyses use only direct comparisons between treatments or diagnostic methods, we also used indirect comparisons. This "network" meta-analysis is a method to quantify a rank order, even if no direct comparison between two methods has been conducted. By using this method we made optimal use of all available data on visual field progression in glaucoma patients. Differences in the incidence proportions that are found with direct comparisons can only be explained by the methods that are applied, because these comparisons are made in the same population. In contrast, indirect comparisons may also be influenced by population differences. Direct comparisons between methods can thus be seen as superior to indirect comparisons. However, 
in this meta-analysis we corrected for follow-up time and baseline MD value, which reduced the unexplained heterogeneity between the incidences to zero. Therefore, we assume that the results of our meta-analysis provide an adequate approximation of direct comparisons between methods that are actually only indirectly compared. An additional analysis using only direct comparisons resulted in approximately the same incidences as when both direct and indirect comparisons were used.

Remarkably, the incidences in the present study are much lower than the incidences of some other well-known studies. These studies were not included in our meta-analysis because they did not directly compare two or more methods. For example, $41 \%$ of the treated patients in EMGT progressed on their visual field in a follow-up period of approximately six years and with a baseline MD value of $-4 \mathrm{~dB}$. ${ }^{35}$ The incidence that we found for the same method (i.e. the GPA method) was only 16\%. This large difference could partly be explained by the frequent testing protocol of the EMGT, in which visual field tests were carried out at 3-month intervals. ${ }^{25}$ Because of the two required confirmations of the GPA method, the availability of at least four visual field tests per year leads to an increased chance to detect progression during the follow-up period. Moreover, a flexible testing protocol resulted in even more available visual fields in the EMGT, because additional visual field tests were performed within one month when tentative progression occurred. As shown in table 2, this testing frequency is much higher than the mean frequency of 1.7 visual fields per year in the studies that were included in our meta-analysis. In addition, all studies in our meta-analysis only used a fixed testing protocol or no testing protocol. Furthermore, the large difference could also be explained by 61 patients in the EMGT who had two eligible eyes for the study. These patients had a higher chance to be progressive because they were considered to have progression when the first eligible eye met the progression criteria. ${ }^{35}$ An additional explanation for the large difference could be that EMGT patients were not treated according to current standards. For example, no target pressure was defined for individual patients. ${ }^{25}$ A higher incidence of progression was also found in the Canadian Glaucoma Study (CGS). ${ }^{36}$ The incidence of visual field progression in this study was $31 \%$ in five years in glaucoma patients with a median baseline MD value of $-4 \mathrm{~dB}$. In the CGS, a variant of method G2 was used with a slightly stricter cut-off point. The estimated incidence of progression of method G2 was only $24 \%$ in our meta-analysis. In view of the better baseline visual field and shorter follow-up period in the CGS compared with the studies in our meta-analysis, one would just expect a lower incidence of progression in the CGS. However, the CGS also used a flexible testing protocol with a testing frequency of at least three visual fields per year and a required confirmation of suspected progression in ten days. ${ }^{36}$ Overall, the studies in our meta-analysis better reflect current clinical practice than the abovementioned studies. We found incidences that are based 
on a heterogeneous group of 948 patients. The mean frequency of visual field examinations of around 1.7 visual fields per year in the present meta-analysis also reflects a feasible testing frequency in clinical practice.

Our study gives insight into the methods that give either high or low estimates of the incidence of glaucomatous visual field progression, but does not solve the problem of the lack of a gold standard. Therefore, the definition of "true progression" remains a problem. A method may well result in a high incidence proportion without really differentiating between "true progressive" and "true non-progressive" patients. A method that correctly classifies a large proportion of the "true progressive" patients as progressive is said to be a method with a high sensitivity. On the other hand, when a method classifies a large proportion of the "true non-progressive" patients as non-progressive, it is said to be a specific method. The choice of a cut-off point in a particular method is generally a trade-off between sensitivity and specificity. In general, we cannot say which method is the better one, but we know that sensitivities and specificities differ per method in a predictable way when another cut-off point is used. When the cut-off value in these methods is less strict, the sensitivity increases while at the same time the specificity decreases. Examples in the present meta-analysis are the PLR-based methods P1, P2, P3, and P4, which only differ in the number of locations that should meet the same criterion. The criterion for a location to be progressive is a statistically significant negative linear regression line $(p \leq 0.001)$. Even without looking at the results of the meta-analysis we can assume that method P1, which needs the highest number of affected locations for a visual field series to be progressive, is the least sensitive and the most specific. The same holds for methods 11, 12, 13, and 14, which differ only in the number of progressive clusters that are required to meet the definition of progression. It is important to notice that in epidemiologic studies, methods with a high specificity are preferred when the true incidence of progression is low. This leads to an estimate of the relative risk of the presumed prognostic factor that is closer to the true value of the relative risk. In clinical practice, a highly specific method with few false positives is also preferred when the treatment of progressive patients has considerable side effects.

We used the results of 30 methods in our meta-analysis, for which a patient could only be progressive or non-progressive. However, some methods were originally intended to quantify the rate of progression instead of a binary presence or absence of progression. These methods are based on indices of the complete tested visual field such as the MD (i.e., in methods 15 and 16) and the VFI (i.e., in method 18). In clinical practice, the MD index and the VFI are used to quantify the rate of progression on a continuous scale, ranging from severe disease progression (e.g., 16\% loss in the VFI per year) to slight improvement (e.g., $3 \%$ increase in the VFI per year). ${ }^{37}$ Sole statistical significance of the 
slopes of these indices may be of limited interest for clinical practice, while knowing the rate of progression would direct appropriately aggressive treatment to those who are at highest risk for visual disability. ${ }^{1}$ However, when a rate of progression is used to decide on changing therapy, it has to be decided which cut-off value for the rate leads to the decision to change therapy. Ultimately, even a rate method leads to a dichotomisation of the population. Rates of progression should therefore be incorporated in progression criteria of methods to determine the presence or absence of progression. For example, progression could be defined as a statistically significant decline of the VFI $(p<0.05)$ with a rate of at least $3 \%$ loss per year. Several PLR methods in the present meta-analysis (i.e., P5, P6, P7, and P8) already used statistically and clinically relevant criteria for individual visual field locations. Finally, the pattern standard deviation (PSD) or the corrected pattern standard deviation (CPSD) indices of the complete tested visual field (e.g., in method 17) should generally not be used in methods to assess glaucomatous visual field progression. Their values shift to normal values again in more advanced glaucoma. $^{38}$

In conclusion, the incidence of glaucomatous visual field progression varies considerably between different studies. This is mainly caused by the variety of methods used to assess progression, but also by differences in follow-up time and baseline visual field loss. Other factors, like the frequency of visual field examinations and the need for confirmation, also seem to be of importance. With a ranking of methods according to their incidence of progression, the interpretation and the possibility to compare studies are improved. Moreover, in several cases the same method can be used with a more or less specific cut-off value, depending on the scientific or clinical goal. An ideal method combines statistically and clinically relevant criteria with tailor-made cut-off values. 


\section{References}

1. Caprioli J. The importance of rates in glaucoma. Am J Ophthalmol 2008;145:191-2.

2. Schulzer M, Anderson DR, Drance SM. Sensitivity and specificity of a diagnostic test determined by repeated observations in the absence of an external standard. J Clin Epidemiol 1991;44:1167-79.

3. Spry PGD, Johnson CA. Identification of progressive glaucomatous visual field loss. Surv Ophthalmol 2002;47:158-73.

4. Ernest PJ, Schouten JS, Beckers HJ, Hendrikse F, Prins MH, Webers CA. The evidence base to select a method for assessing glaucomatous visual field progression. Acta Ophthalmol 2012;90:101-8.

5. Bucher HC, Guyatt GH, Griffith LE, Walter SD. The results of direct and indirect treatment comparisons in meta-analysis of randomized controlled trials. J Clin Epidemiol 1997;50:683-91.

6. Mayama C, Araie M, Suzuki Y, Ishida K, Yamamoto T, Kitazawa Y, et al. Statistical evaluation of the diagnostic accuracy of methods used to determine the progression of visual field defects in glaucoma. Ophthalmology 2004;111:2117-25.

7. Heijl A, Bengtsson B, Chauhan BC, Lieberman MF, Cunliffe I, Hyman L, et al. A comparison of visual field progression criteria of 3 major glaucoma trials in early manifest glaucoma trial patients. Ophthalmology 2008;115:1557-65.

8. Caldwell DM, Ades AE, Higgins JP. Simultaneous comparison of multiple treatments: combining direct and indirect evidence. BMJ 2005;331:897-900.

9. Arnalich Montiel F, Casas Llera P, Munoz Negrete FJ, Rebolleda G. Performance of glaucoma progression analysis software in a glaucoma population. Graefes Arch Clin Exp Ophthalmol 2009;247:391-7.

10. Artes PH, Nicolela MT, LeBlanc RP, Chauhan BC. Visual field progression in glaucoma: total versus pattern deviation analyses. Invest Ophthalmol Vis Sci 2005;46:4600-6.

11. Birch MK, Wishart PK, O'Donnell NP. Determining progressive visual field loss in serial Humphrey visual fields. Ophthalmology 1995;102:1227-34; discussion 34-5.

12. Katz J. A comparison of the pattern- and total deviation-based Glaucoma Change Probability programs. Invest Ophthalmol Vis Sci 2000;41:1012-6.

13. Katz J, Congdon N, Friedman DS. Methodological variations in estimating apparent progressive visual field loss in clinical trials of glaucoma treatment. Arch Ophthalmol 1999;117:1137-42.

14. Katz J, Gilbert D, Quigley HA, Sommer A. Estimating progression of visual field loss in glaucoma. Ophthalmology 1997;104:1017-25.

15. Nouri Mahdavi K, Hoffman D, Ralli M, Caprioli J. Comparison of methods to predict visual field progression in glaucoma. Arch Ophthalmol 2007;125:1176-81.

16. Smith SD, Katz J, Quigley HA. Analysis of progressive change in automated visual fields in glaucoma. Invest Ophthalmol Vis Sci 1996;37:1419-28.

17. Diaz Aleman VT, Anton A, de la Rosa MG, Johnson ZK, McLeod S, Azuara Blanco A. Detection of visual-field deterioration by Glaucoma Progression Analysis and Threshold Noiseless Trend programs. $\mathrm{Br} J$ Ophthalmol 2009;93:322-8.

18. Lee AC, Sample PA, Blumenthal EZ, Berry C, Zangwill L, Weinreb RN. Infrequent confirmation of visual field progression. Ophthalmology 2002;109:1059-65.

19. Wesselink C, Heeg GP, Jansonius NM. Glaucoma monitoring in a clinical setting: Glaucoma progression analysis vs nonparametric progression analysis in the Groningen longitudinal glaucoma study. Arch Ophthalmol 2009;127:270-4.

20. Viswanathan AC, Crabb DP, McNaught Al, Westcott MC, Kamal D, Garway Heath DF, et al. Interobserver agreement on visual field progression in glaucoma: A comparison of methods. $\mathrm{Br} J$ Ophthalmol 2003;87:726-30. 
21. Casas Llera P, Rebolleda G, Munoz Negrete FJ, Arnalich Montiel F, Perez Lopez M, Fernandez Buenaga R. Visual field index rate and event-based glaucoma progression analysis: comparison in a glaucoma population. Br J Ophthalmol 2009;93:1576-9.

22. Salanti G, Higgins JP, Ades AE, loannidis JP. Evaluation of networks of randomized trials. Stat Methods Med Res 2008;17:279-301.

23. Riley RD, Thompson JR, Abrams KR. An alternative model for bivariate random-effects meta-analysis when the within-study correlations are unknown. Biostatistics 2008;9:172-86.

24. Hardy RJ, Thompson SG. A likelihood approach to meta-analysis with random effects. Stat Med 1996;15:619-29.

25. Leske MC, Heijl A, Hyman L, Bengtsson B. Early Manifest Glaucoma Trial: design and baseline data. Ophthalmology 1999;106:2144-53.

26. The AGIS Investigators. Advanced Glaucoma Intervention Study. 2. Visual field test scoring and reliability. Ophthalmology 1994;101:1445-55.

27. Katz J. Scoring systems for measuring progression of visual field loss in clinical trials of glaucoma treatment. Ophthalmology 1999;106:391-5.

28. Bengtsson B, Heijl A. A visual field index for calculation of glaucoma rate of progression. Am J Ophthalmol 2008;145:343-53.

29. Gonzalez De La Rosa M, Gonzalez Hernandez M, Diaz Aleman T. Linear regression analysis of the cumulative defect curve by sectors and other criteria of glaucomatous visual field progression. Eur J Ophthalmol 2009;19:416-24.

30. Boden C, Blumenthal EZ, Pascual J, McEwan G, Weinreb RN, Medeiros F, et al. Patterns of glaucomatous visual field progression identified by three progression criteria. Am J Ophthalmol 2004;138:1029-36.

31. Leske MC, Heijl A, Hyman L, Bengtsson B, Dong L, Yang Z. Predictors of long-term progression in the early manifest glaucoma trial. Ophthalmology ISE: 15494713 2007;114:1965-72.

32. Lichter PR, Musch DC, Gillespie BW, Guire KE, Janz NK, Wren PA, et al. Interim clinical outcomes in the collaborative initial glaucoma treatment study comparing initial treatment randomized to medications or surgery. Ophthalmology 2001;108:1943-53.

33. Polansky JR, Juster RP, Spaeth GL. Association of the myocilin mt.1 promoter variant with the worsening of glaucomatous disease over time. Clin Genet 2003;64:18-27.

34. The AGIS Investigators. The Advanced Glaucoma Intervention Study (AGIS): 7. The relationship between control of intraocular pressure and visual field deterioration. Am J Ophthalmol 2000;130:429-40.

35. Heijl A, Leske MC, Bengtsson B, Hyman L, Bengtsson B, Hussein M. Reduction of intraocular pressure and glaucoma progression: results from the Early Manifest Glaucoma Trial. Arch Ophthalmol 2002;120:126879.

36. Chauhan BC, Mikelberg FS, Balaszi AG, LeBlanc RP, Lesk MR, Trope GE. Canadian Glaucoma Study: 2. risk factors for the progression of open-angle glaucoma. Arch Ophthalmol 2008;126:1030-6.

37. Bengtsson B, Patella VM, Heijl A. Prediction of glaucomatous visual field loss by extrapolation of linear trends. Arch Ophthalmol 2009;127:1610-5.

38. Heijl A, Lindgren G, Olsson J. A package for the statistical analysis of visual fields. In: Doc Ophthalmol Proc Ser. Dordrecht: Martinus Nijhoff Publishers, 1987. 


\section{CHAPTER 5}

\section{The evidence base to select a method for assessing glaucomatous visual field progression}

Authors:

Paul J.G. Ernest

Jan S.A.G. Schouten

Henny J.M. Beckers

Fred Hendrikse

Martin H. Prins

Carroll A.B. Webers

Acta Ophthalmologica 2012;90:101-108 


\section{ABSTRACT}

A large number of methods have been developed for assessing glaucomatous visual field progression, but their properties have not yet been systematically evaluated. In this systematic literature review we summarize the evidence base for selecting a method by providing answers to ten relevant questions on the variety, validity, and reproducibility of methods. In total, we found 301 different methods in 412 articles. The majority of studies (54\%) used the Humphrey Field Analyzer. No data have been published about the reproducibility of methods. Although there is no gold standard to assess glaucomatous visual field progression, we found evidence on validity for 48 different methods. Some methods were less capable of distinguishing between progressive and nonprogressive patients. Choosing among twelve methods is supported by some evidence of their validity. These methods still differ in sensitivity, specificity, and predictive values of test results within studies comparing several methods. In conclusion, the current evidence base is not perfect. A selection should be made from a limited number of methods, according to the clinical purpose of progression assessment. Methods that quantify the rate of visual field progression seem to be the most appropriate for guiding subsequent medical actions in individual patients. Future studies should investigate whether using one method to monitor patients is superior to another method in preventing loss of quality of life. 


\section{Introduction}

An important goal in the management of open-angle glaucoma is to detect clinically relevant visual field progression early and reliably. The methods to assess progression however, are a continuing subject of debate..$^{1-6}$ In the last years, the relevance of monitoring the rate of visual field progression has increasingly being recognized. ${ }^{7}$ Together with the patient's life expectancy and disease stage, the rate of progression can be used to estimate individual risk of lifetime visual disability. ${ }^{8}$ However, from all methods to determine visual field progression it may still be difficult to select a specific one.

This paper provides a systematic review of the evidence base for selecting a method to assess glaucomatous visual field progression. The clinical use of a good method to assess progression should ultimately optimize quality of life (QoL) of glaucoma patients, without under- or overtreatment. With this aim in mind we searched the literature to provide answers to ten relevant questions.

\section{Ten questions and answers}

\section{How many methods can we choose from to assess visual field progression?}

A total of 301 different methods were used in 412 articles.

We classified all methods that we found in the literature. There are 15 different perimeters used to assess progression in the literature. A majority of 222 studies (54\%) used the Humphrey Field Analyzer (HFA) (Carl Zeiss Meditec, Dublin, CA, USA), increasing to $77 \%$ of the articles published since 2000 . We therefore focused on methods for the HFA for the rest of this article. HFA methods could further be classified into qualitative and quantitative methods. A qualitative method implies that the ophthalmologist decides on the occurrence of progression, whereas a quantitative method uses numeric units for defining progression. Qualitative methods have been used 32 times (8\%) and quantitative methods 355 times (92\%). Quantitative methods which calculate a rate of progression were used 166 times (47\% of quantitative methods). However, most of these studies dichotomized the rate of progression because they aimed to compare different progression methods or estimated treatment effects in a large group of patients. Therefore, even these methods did not really quantify the rate of progression needed for decision making in individual patients. 


\section{Which method to assess visual field progression can predict loss of QoL?}

The prediction of loss in QoL has not been shown for any method.

The ultimate goal of glaucoma management is to prevent loss of QoL. A method to assess progression should therefore identify patients who will lose vision related QoL in the future if treatment is not intensified. Although this constitutes the essential goal of monitoring progression it has not been addressed in empirical research. However, its clinical relevance is increasingly being recognized. ${ }^{7}$ Empirical research should ideally randomize patients to different monitoring strategies with a subsequent long follow-up period to evaluate differences in QoL. Future studies should address this issue with the inclusion of methods quantifying the rate of progression. What we do know is that the degree of visual field loss and QoL are strongly related. ${ }^{9-13}$

\section{What is the gold standard to assess glaucomatous visual field progression?}

There is no gold standard to assess visual field progression.

A gold standard is a method that is closest to anatomical and consequent functional changes, against which new developments should be compared. ${ }^{14}$ There is no universally accepted solution in diagnostic research when faced with a missing or an imperfect gold standard. ${ }^{15}$ In the literature about methods to assess glaucomatous visual field progression, most studies compared incidences of progression from different methods. Although these studies give insight into the methods that give either high or low estimates of the incidence of progression, they do not contribute in the selection of the best method. We summarized the results of these studies in an earlier review article. ${ }^{16}$ Other studies dealt with the problem of the lack of a gold standard by using another reference standard against which methods were compared. Different reference standards have been used that are of some value to substitute for a gold standard.

\section{Which methods have been compared with a substitute gold standard of visual field progression or stability?}

Several methods have been compared with a substitute gold standard to assess visual field progression. In the field of clinimetrics this is termed concurrent validity. ${ }^{17}$ The resulting sensitivities, specificities, positive predictive values (PPVs), negative predictive values (NPVs), likelihood ratios (LRs), and diagnostic odds ratios are shown in Table $1^{1-6 \text {, }}$ $18-28,30$ The first thing to note is that there is a lot of variation in several accuracy measures within studies and between studies. There seems to be no superior method, although some have a lower diagnostic odds ratio when compared with other methods within one study. These methods are less capable to distinguish between progressive and non-progressive patients.

Some studies made use of expert judgment of visual fields series as a substitute for a gold standard. 3, 6, 19, 20 However, these qualitative judgments can be seen as arbitrary 
gold standards, because they have not shown to be superior to other methods. If subjective judgments are used, it is important that these judgments are masked for other clinical information. This was the case in all studies. Three studies calculated sensitivities and specificities with use of a preselected sample of unequivocally progressive and non-progressive patients after excluding patients with equivocal results., 3,20 This may have resulted in overestimated accuracy measures.

Table 1: Accuracy measures of different methods to assess visual field progression

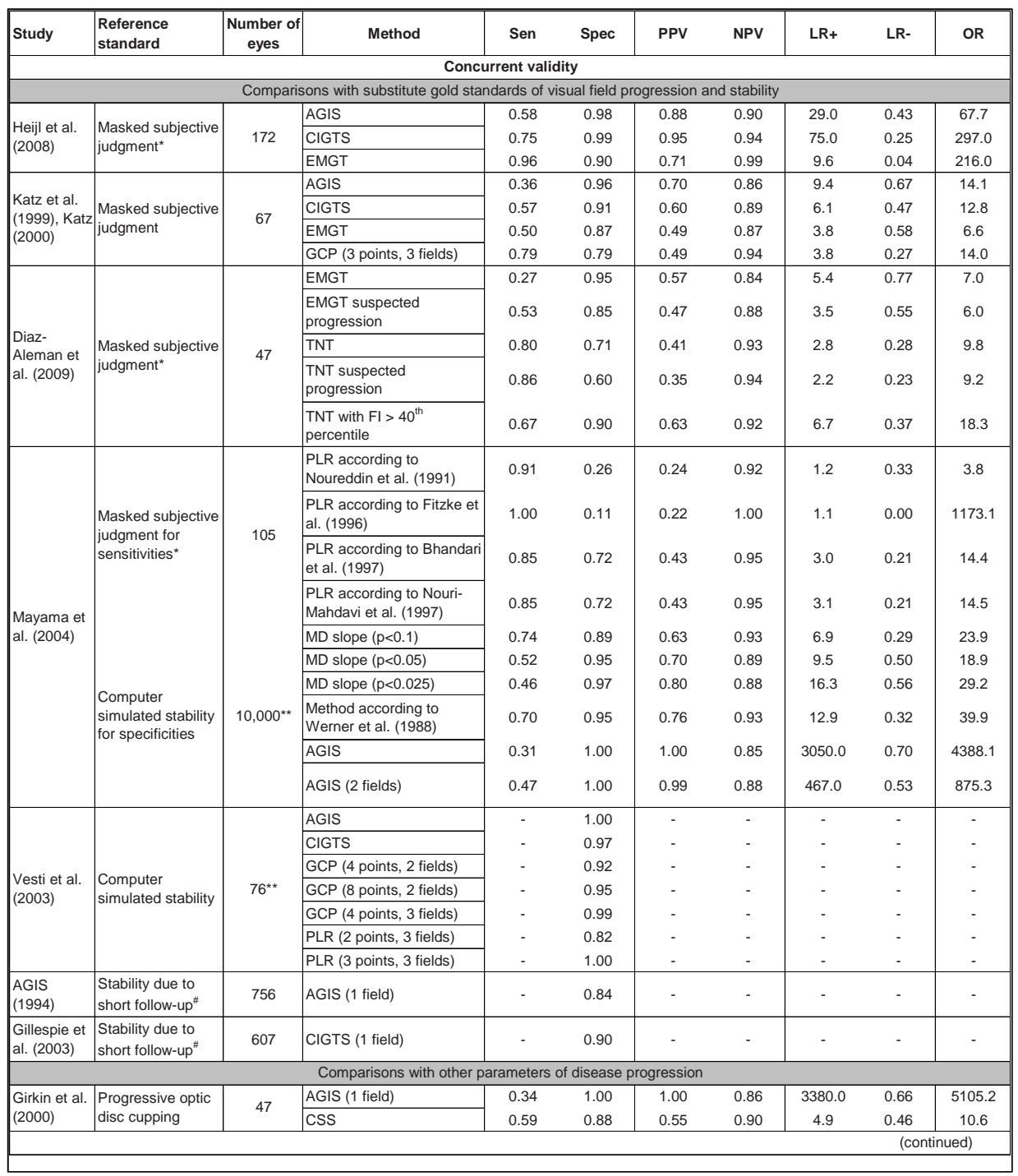


Table 1: continued

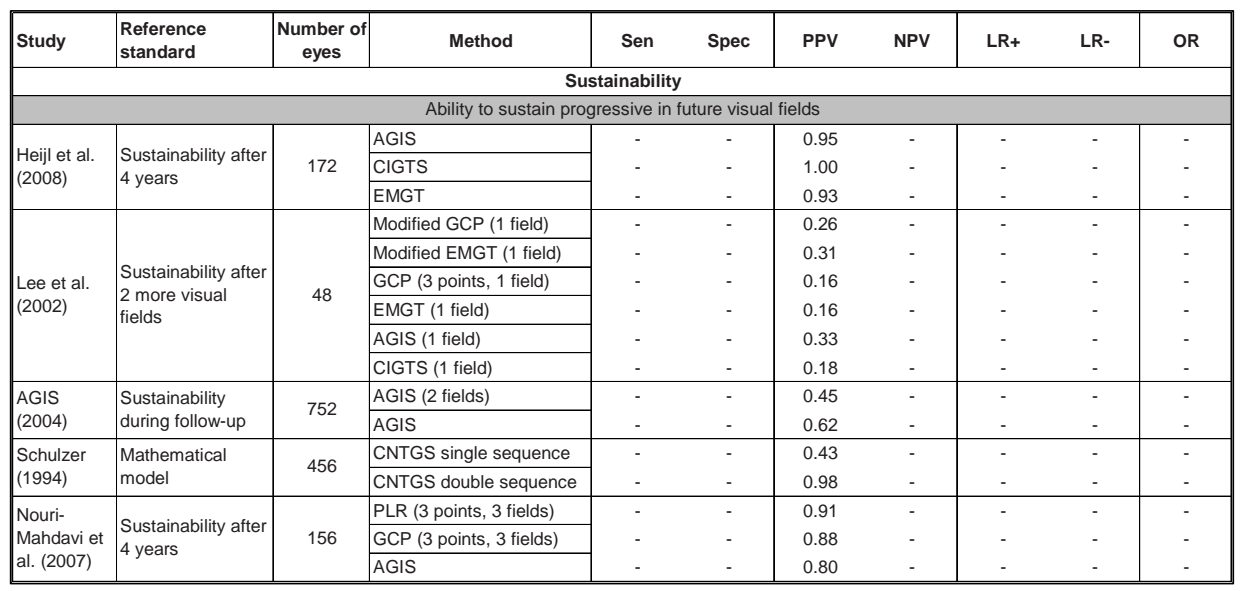

If sensitivities and specificities of methods were available, we calculated positive and negative predictive values, likelihood ratios, and odds ratios using formulas that have earlier been described. ${ }^{29}$ Positive and negative predictive values were calculated for a presumed incidence of progression of $20 \%$ in six years, based on a recent meta-analysis. ${ }^{16}$ Cut-off points for several methods are shown between brackets (e.g. the number of test points or visual fields that had to be progressive before the total visual field series is considered to be progressive).

* These studies used a preselected sample of unequivocally progressive and non-progressive patients after excluding patients with equivocal results.

** Simulated visual field series.

\# Stable eyes are compared with eyes changed in either direction.

Sen = sensitivity, $\mathrm{Spec}=$ specificity, PPV = positive predictive value, which is the probability that a patient has "true progression" when the method indicates a patient as progressive, NPV = negative predictive value, which is the probability that the patient is "true non-progressive" when the method indicates a patient as non-progressive, $L R+=$ likelihood ratio of a positive test result, which indicates how much a positive test result will raise the pretest probability of progression, LR- = likelihood ratio of a negative test result, which indicates how much a negative test result will lower the pretest probability of progression, OR = diagnostic odds ratio, which describes the odds of a positive test result in patients with progression compared with the odds of a positive test result in those without progression, AGIS = Advanced Glaucoma Intervention Study, CIGTS = Collaborative Initial Glaucoma Treatment Study, EMGT = Early Manifest Glaucoma Trial, GCP = Glaucoma Change Probability, TNT $=$ Threshold Noiseless Trend program, FI = focality index, PLR = pointwise linear regression analysis, $\mathrm{MD}=$ Mean Deviation, $\mathrm{CSS}=$ clinical scoring system based on scotomas, CNTGS = Collaborative Normal-Tension Glaucoma Study

As another substitute for a gold standard, some studies used groups of glaucoma patients that were likely to be stable (i.e. not showing progression) to calculate the method's specificity. ${ }^{3}$, 5, 18, 21 Using a computer simulation, two studies constructed a substitute gold standard by simulating stable visual field series with a physiological degree of variability., 5 
Two other studies approached a method's specificity by testing if progression criteria were fulfilled within a follow-up period that was too short for the visual field to change (i.e. one month). ${ }^{18,21}$

\section{Which methods have been compared with other parameters of disease progression?}

We found one study that used progressive optic disc cupping as a reference standard. The Advanced Glaucoma Intervention Study (AGIS) method had a very high diagnostic odds ratio and a very high likelihood ratio for a positive test result (Table 1 ). ${ }^{22}$

Instead of comparing methods to assess glaucomatous visual field progression with a substitute gold standard to assess visual field progression, outcomes of methods can also be compared with other parameters of disease progression like changes of the optic nerve head. This is also a form of concurrent validity.

\section{Which methods give a good prediction of future visual field loss?}

Several methods have a high sustainability. These include the AGIS, Collaborative Initial Glaucoma Treatment Study (CIGTS), Early Manifest Glaucoma Trial (EMGT), Collaborative Normal-Tension Glaucoma Study (CNTGS), and pointwise linear regression analysis (PLR) methods (Table 1).

When methods have shown progression, they should remain to do so when additional follow-up visual fields are acquired since glaucomatous nerve fibre loss is assumed to be irreversible. The positive test results must therefore be sustained in future assessments. However, the results of assessments that do not show progression are not supposed to sustain since the patient could progress afterwards. In clinimetrics this is termed predictive validity, but sustainability is a more appropriate term as used by the authors. ${ }^{17}$

One way to investigate the sustainability of progression is to use the outcomes after a limited number of follow-up years to predict outcomes after a longer period, both using the same baseline as a reference. ${ }^{1,6,24,30}$ Furthermore, another study estimated the sustainability of the CNTGS method by a mathematical model based on repeated testing. ${ }^{23}$

Instead of looking at the sustainability of positive test results, Bengtsson and co-workers used correlations to validate the continuous Visual Field Index (VFI) rate. ${ }^{31}$ They investigated if the VFI rate in the initial 3.3 years could reliably predict the VFI after a mean follow-up time of 8.2 years. A correlation coefficient of 0.78 was found when the predicted VFI was compared with the actual last VFI. ${ }^{31}$ 


\section{Which methods have shown to be related with a presumed prognostic factor of glaucomatous progression?}

In total, 20 different methods have been studied in relation with mean intraocular pressure (IOP) in 21 articles. Thirteen methods (65\%) found a positive relation between mean IOP and glaucomatous visual field progression (Fig. 1). ${ }^{25,32-52}$ Six of these methods $(30 \%)$ showed a statistically significant positive difference $(p<0.05)$ in mean IOP between the progressive and non-progressive groups, including the EMGT method, ${ }^{32}$ another method based on pointwise event analysis, ${ }^{40}$ a method based on $\mathrm{PLR}^{43}$ and three qualitative methods ${ }^{37,46,47}$ (Fig. 1).

A method that is assumed to assess glaucomatous visual field progression should be able to find a relation between progression and a presumed risk factor for glaucomatous progression. In clinimetrics this is termed construct validity. ${ }^{53}$ An example could be the relation with mean IOP during the follow-up period. The reason for this is that IOP is a consistent prognostic factor for glaucoma and the focus of treatment. ${ }^{54}$ Although the four qualitative methods resulted in a high positive association between mean IOP and visual field progression, the outcome assessment in these studies was not masked for other clinical information. 33, 37, 46, 47 Moreover, two of them included other parameters like visual acuity and optic disc criteria in the judgment of progression. ${ }^{46,47}$

\section{Which methods have shown to be reproducible?}

To our knowledge, no studies about the reproducibility of methods to assess visual field progression have been conducted.

However, we found 21 articles that studied cross-sectional reproducibility of visual field measures that were derived from the HFA. ${ }^{21,55-74}$ In general, these studies showed that Mean Deviation (MD) values have a higher reproducibility than pointwise values.

\section{Taking into account the evidence above, which method should we select from the 301 available methods?}

The selection from 301 methods is limited to 48 different methods for which data on validity was present (see questions 4-7). Excluding the different cut-off points, the selection is limited to twelve methods being the AGIS, CIGTS, PLR, MD, Glaucoma Change Probability (GCP), EMGT, VFI, Threshold Noiseless Trend (TNT), Werner, clinical scoring system (CSS), CNTGS, and subjective methods. It is important to keep in mind that methods were validated with use of different reference standards and different study designs. 
Methods based on the AGIS generally have a high discriminative ability, which is shown by the high odds ratios in Table 1. However, we found an inverse relation between AGIS progression and mean IOP during follow-up (Fig. 1). This is probably a biased result since IOP may have been lowered due to changes in treatment among those with progression. Moreover, mean IOP during the first follow-up years has earlier shown to be associated with subsequent progression on the AGIS score. ${ }^{75}$ The CIGTS method also showed high odds ratios and PPVs. Methods with a high PPV, like the AGIS and the CIGTS methods, could for example be used before performing glaucoma surgery, where one wants to be certain that a patient is really progressing.

PLR methods showed highly variable accuracy measures ranging from a low odds ratio of 3.8 to a much better odds ratio of 1173.1 (Table 1). This is caused by the use of many different cut-off points for these methods in the literature. However, PLR, AGIS, or CIGTS methods may be impractical in clinical practice, because they are more timeconsuming due to the need to interpret several test locations. These methods could possibly be made more usable by computerization of the analysis.

Methods based on the VFI, MD, GCP, or EMGT may be more usable, because the required information is available on the printed output of the HFA. Among them, the EMGT method is the only method that has shown to correlate with mean IOP during follow-up (Fig. 1). Methods based on MD and EMGT seem to perform well in the studies of Heijl and Mayama, although these studies probably overestimated the method's accuracy (Table 1). ${ }^{3,6}$ The odds ratio of the EMGT method was relatively low in the other studies. $^{19,20}$

Qualitative methods could also be useful, although the interpretation of results is dependent on the capacity of the observer. This may cause high inter-observer variability. ${ }^{28}$ Nonetheless, these methods have frequently been used as a substitute for a gold standard. In these cases, however, the assessment was based on the judgement of more than one observer. Qualitative methods have also shown to correlate well with mean IOP, but these findings could be biased because these qualitative assessments were not masked for other clinical information.

\section{In the end, what do we really want to know?}

The current evidence base is not perfect but seems to be fair for a few methods that have been validated. Since numerous methods are available, we should probably stop developing many new methods to assess visual field progression. The ultimately relevant question, whether using one method to monitor patients is superior to another in preventing loss of QoL, has not been answered. Methods that quantify the rate of visual 
field progression seem to be the most appropriate for guiding subsequent medical actions in individual patients, because they can be used to estimate individual risk of lifetime visual disability. This should ideally be studied in prospective studies with long follow-up periods. If this is not feasible, new research should be directed to other types of validation, like the ability of methods to predict future visual field loss and to the relation between outcomes of methods and structural progression or prognostic factors. 


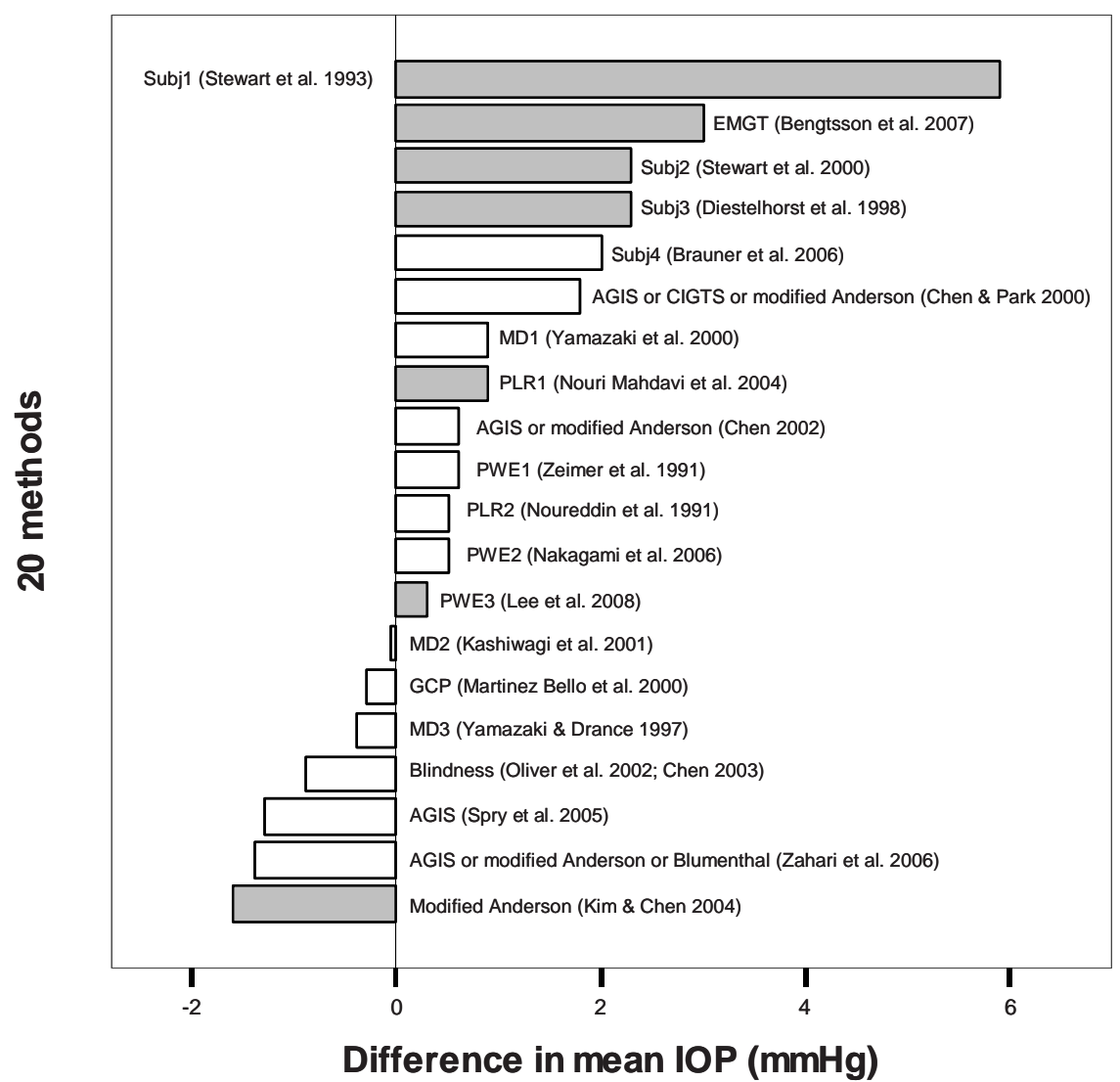

Figure 1: Difference in mean intraocular pressure between progressive and non-progressive patients according to 20 different methods to assess progression

A positive difference indicates a positive relation between visual field progression and mean intraocular pressure during follow-up. The grey bars represent the differences that were tested statistically significant while the white bars represent the differences that were not statistically significant. Each bar is labelled by the method that was used and by a reference of the study from where we derived the data. When one method was investigated in two studies, we calculated the weighted mean difference between the progressive and non-progressive groups based on the number of patients in each study. Bars Subj1 to Subj4 represent qualitative (i.e. subjective) methods, bars MD1 to MD3 represent methods based on the Mean Deviation index, bars PLR1 to PLR2 represent methods based on pointwise linear regression analysis, and bars PWE1 to PWE3 represent methods based on pointwise event analysis. Modified Anderson represents a method adapted from Chen and Park. ${ }^{36}$ Blindness represents a method that uses blindness as end-point. Blumenthal represents a method derived from Blumenthal and co-workers. ${ }^{52}$

$\mathrm{IOP}=$ intraocular pressure, EMGT = Early Manifest Glaucoma Trial method, $\mathrm{AGIS}=$ Advanced Glaucoma Intervention Study method, CIGTS = Collaborative Initial Glaucoma Treatment Study method, GCP = Glaucoma Change Probability method 


\section{Literature search}

To get an overview of all methods to assess glaucomatous visual field progression, we performed a systematic literature search in April 2009. We searched in PubMed, EMBASE, and all databases and registers of The Cochrane Library with use of the following keywords: (Glaucoma*) AND (Prognos* OR Predict* OR Progress* OR Longitudinal OR Cohort OR Follow-up) AND (Perimetr* OR Visual Field* OR HFA OR Octopus OR Humphrey (not in author)). A total of 2,450 articles were identified. Based on predefined exclusion criteria, we selected studies reporting on glaucoma patients who were followed for minimally one year with the use of standard visual field examinations, so that progression could be assessed (Table 2). We finally included 412 articles (Fig. 2). From this search, we also selected 21 articles that used the HFA and studied mean follow-up IOP as a prognostic factor for glaucomatous visual field progression.

We studied the reproducibility of progression methods in glaucoma patients by performing a second systematic search in PubMed in April 2009: Glaucoma [Mesh] AND Visual fields [Mesh] AND (Observer Variation [Mesh] OR Reproducibility of Results [Mesh]).

Table 2: Number of articles excluded according to different criteria

\begin{tabular}{ll}
\hline Exclusion criteria & Number \\
\hline No intention to measure progression of VF damage & 471 \\
Unconventional or single perimetric measurement & 434 \\
No visual field damage at the beginning of follow-up & 395 \\
Patients without glaucomatous disease & 244 \\
No original study & 172 \\
Perimetric follow-up <1 year (median or mean <1 year) & 124 \\
Case-report (<10 patients) & 80 \\
Patients aged <18 years & 35 \\
Animal study & 17 \\
Other languages than English, French, Dutch, or German & 8 \\
\hline
\end{tabular}

Ten exclusion criteria are listed with their accompanying number of excluded articles.

$\mathrm{VF}=$ visual field 
Potential relevant articles identified and screened for retrieval $(n=2450)$

Articles excluded based on title and abstract $(n=1668)$

Articles selected for more detailed information $(n=782)$

Abstracts $(n=23)$

Articles not available in the Netherlands $(n=35)$

Articles excluded based on full text $(n=312)$

Articles included in systematic review $(n=412)$

Articles using a HFA $(n=222)$

Figure 2: Flow chart of studies

The selection process of studies found in the first systematic literature review is shown in this flow chart. Four hundred twelve articles were finally included. HFA = Humphrey Field Analyzer 


\section{References}

1. Lee AC, Sample PA, Blumenthal EZ, Berry C, Zangwill L, Weinreb RN. Infrequent confirmation of visual field progression. Ophthalmology 2002;109:1059-65.

2. Katz J, Congdon N, Friedman DS. Methodological variations in estimating apparent progressive visual field loss in clinical trials of glaucoma treatment. Arch Ophthalmol 1999;117:1137-42.

3. Mayama C, Araie M, Suzuki Y, Ishida K, Yamamoto T, Kitazawa Y, et al. Statistical evaluation of the diagnostic accuracy of methods used to determine the progression of visual field defects in glaucoma. Ophthalmology 2004;111:2117-25.

4. Nouri Mahdavi K, Brigatti L, Weitzman M, Caprioli J. Comparison of methods to detect visual field progression in glaucoma. Ophthalmology 1997;104:1228-36.

5. Vesti E, Johnson CA, Chauhan BC. Comparison of different methods for detecting glaucomatous visual field progression. Invest Ophthalmol Vis Sci 2003;44:3873-9.

6. Heijl A, Bengtsson B, Chauhan BC, Lieberman MF, Cunliffe I, Hyman L, et al. A Comparison of Visual Field Progression Criteria of 3 Major Glaucoma Trials in Early Manifest Glaucoma Trial Patients. Ophthalmology 2008;115:1557-65.

7. Rossetti L, Goni F, Denis P, Bengtsson B, Martinez A, Heijl A. Focusing on glaucoma progression and the clinical importance of progression rate measurement: a review. Eye (Lond) 2010;24 Suppl 1:S1-7.

8. Bengtsson B, Heijl A. A visual field index for calculation of glaucoma rate of progression. Am J Ophthalmol 2008;145:343-53.

9. McKean Cowdin R, Wang Y, Wu J, Azen SP, Varma R. Impact of visual field loss on health-related quality of life in glaucoma: the Los Angeles Latino Eye Study. Ophthalmology 2008;115:941-8.

10. Viswanathan AC, McNaught AI, Poinoosawmy D, Fontana L, Crabb DP, Fitzke FW, et al. Severity and stability of glaucoma: patient perception compared with objective measurement. Arch Ophthalmol 1999;117:450-4.

11. Gutierrez P, Wilson MR, Johnson C, Gordon M, Cioffi GA, Ritch R, et al. Influence of glaucomatous visual field loss on health-related quality of life. Arch Ophthalmol 1997;115:777-84.

12. Kobelt G, Jonsson B, Bergstrom A, Chen E, Linden C, Alm A. Cost-effectiveness analysis in glaucoma: what drives utility? Results from a pilot study in Sweden. Acta Ophthalmol Scand 2006;84:363-71.

13. van Gestel A, Webers CA, Beckers HJ, van Dongen MC, Severens JL, Hendrikse F, et al. The relationship between visual field loss in glaucoma and health-related quality-of-life. Eye (Lond) 2010;24:1759-69.

14. Higgins JPT, Green S, eds. Glossary. Cochrane Handbook for Systematic Reviews of Interventions 4.2.5. The Cochrane Collaboration; 2005.. Available at: http://www.cochrane.org/resources/handbook/. Accessed May 18, 2010.

15. Reitsma JB, Rutjes AW, Khan KS, Coomarasamy A, Bossuyt PM. A review of solutions for diagnostic accuracy studies with an imperfect or missing reference standard. J Clin Epidemiol 2009;62:797-806.

16. Ernest PJ, Viechtbauer W, Schouten JS, Beckers HJ, Hendrikse F, Prins MH, et al. The influence of the assessment method on the incidence of visual field progression in glaucoma: a network meta-analysis. Acta Ophthalmol Scand 2012;90:10-9.

17. Streiner DL, Norman GR. Health measurement scales: a practical guide to their development and use. Oxford: Oxford University Press; 2008:254.

18. The AGIS Investigators. Advanced Glaucoma Intervention Study. 2. Visual field test scoring and reliability. Ophthalmology 1994;101:1445-55.

19. Katz J. A comparison of the pattern- and total deviation-based Glaucoma Change Probability programs. Invest Ophthalmol Vis Sci 2000;41:1012-6.

20. Diaz Aleman VT, Anton A, de la Rosa MG, Johnson ZK, McLeod S, Azuara Blanco A. Detection of visual-field deterioration by Glaucoma Progression Analysis and Threshold Noiseless Trend programs. $\mathrm{Br} J$ Ophthalmol 2009;93:322-8. 
21. Gillespie BW, Musch DC, Guire KE, Mills RP, Lichter PR, Janz NK, et al. The collaborative initial glaucoma treatment study: baseline visual field and test-retest variability. Invest Ophthalmol Vis Sci 2003;44:261320.

22. Girkin CA, Emdadi A, Sample PA, Blumenthal EZ, Lee AC, Zangwill LM, et al. Short-wavelength automated perimetry and standard perimetry in the detection of progressive optic disc cupping. Arch Ophthalmol 2000;118:1231-6.

23. Schulzer M. Errors in the diagnosis of visual field progression in normal-tension glaucoma. Ophthalmology 1994;101:1589-94.

24. Nouri Mahdavi K, Hoffman D, Ralli M, Caprioli J. Comparison of methods to predict visual field progression in glaucoma. Arch Ophthalmol 2007;125:1176-81.

25. Noureddin BN, Poinoosawmy D, Fietzke FW, Hitchings RA. Regression analysis of visual field progression in low tension glaucoma. Br J Ophthalmol 1991;75:493-5.

26. Fitzke FW, Hitchings RA, Poinoosawmy D, McNaught Al, Crabb DP. Analysis of visual field progression in glaucoma. Br J Ophthalmol 1996;80:40-8.

27. Bhandari A, Crabb DP, Poinoosawmy D, Fitzke FW, Hitchings RA, Noureddin BN. Effect of surgery on visual field progression in normal-tension glaucoma. Ophthalmology 1997;104:1131-7.

28. Werner EB, Bishop KI, Koelle J, Douglas GR, LeBlanc RP, Mills RP, et al. A comparison of experienced clinical observers and statistical tests in detection of progressive visual field loss in glaucoma using automated perimetry. Arch Ophthalmol 1988;106:619-23.

29. Knottnerus JA. The evidence base of clinical diagnosis. London: BMJ Books; 2002:125-32.

30. The AGIS Investigators. The Advanced Glaucoma Intervention Study (AGIS): 14. Distinguishing progression of glaucoma from visual field fluctuations. Ophthalmology 2004;111:2109-16.

31. Bengtsson B, Patella VM, Heijl A. Prediction of glaucomatous visual field loss by extrapolation of linear trends. Arch Ophthalmol 2009;127:1610-5.

32. Bengtsson B, Leske MC, Hyman L, Heijl A. Fluctuation of intraocular pressure and glaucoma progression in the early manifest glaucoma trial. Ophthalmology 2007;114:205-9.

33. Brauner SC, Chen TC, Hutchinson BT, Chang MA, Pasquale LR, Grosskreutz CL. The course of glaucoma during pregnancy: a retrospective case series. Arch Ophthalmol 2006;124:1089-94.

34. Chen PP. Correlation of visual field progression between eyes in patients with open-angle glaucoma. Ophthalmology 2002;109:2093-9.

35. Chen PP. Blindness in patients with treated open-angle glaucoma. Ophthalmology 2003;110:726-33.

36. Chen PP, Park RJ. Visual field progression in patients with initially unilateral visual field loss from chronic open-angle glaucoma. Ophthalmology 2000;107:1688-92.

37. Diestelhorst M, Khalili MA, Krieglstein GK. Trabeculectomy: a retrospective follow-up of 700 eyes. Int Ophthalmol 1998;22:211-20.

38. Kashiwagi K, Hosaka O, Kashiwagi F, Taguchi K, Mochizuki J, Ishii H, et al. Systemic circulatory parameters. comparison between patients with normal tension glaucoma and normal subjects using ambulatory monitoring. Jpn J Ophthalmol 2001;45:388-96.

39. Kim JW, Chen PP. Central corneal pachymetry and visual field progression in patients with open-angle glaucoma. Ophthalmology 2004;111:2126-32.

40. Lee YA, Shih YF, Lin LLK, Huang JY, Wang TH. Association between high myopia and progression of visual field loss in primary open-angle glaucoma. J Formos Med Assoc 2008;107:952-7.

41. Martinez Bello C, Chauhan BC, Nicolela MT, McCormick TA, Leblanc RP. Intraocular pressure and progression of glaucomatous visual field loss. Am J Ophthalmol 2000;129:302-8.

42. Nakagami T, Yamazaki Y, Hayamizu F. Prognostic factors for progression of visual field damage in patients with normal-tension glaucoma. Jpn J Ophthalmol 2006;50:38-43. 
43. Nouri Mahdavi K, Hoffman D, Coleman AL, Liu G, Li G, Gaasterland D, et al. Predictive factors for glaucomatous visual field progression in the Advanced Glaucoma Intervention Study. Ophthalmology 2004;111:1627-35.

44. Oliver JE, Hattenhauer MG, Herman D, Hodge DO, Kennedy R, Fang Yen M, et al. Blindness and glaucoma: a comparison of patients progressing to blindness from glaucoma with patients maintaining vision. Am J Ophthalmol 2002;133:764-72.

45. Spry PG, Sparrow JM, Diamond JP, Harris HS. Risk factors for progressive visual field loss in primary open angle glaucoma. Eye 2005;19:643-51.

46. Stewart WC, Chorak RP, Hunt HH, Sethuraman G. Factors associated with visual loss in patients with advanced glaucomatous changes in the optic nerve head. Am J Ophthalmol 1993;116:176-81.

47. Stewart WC, Kolker AE, Sharpe ED, Day DG, Holmes KT, Leech JN, et al. Factors associated with long-term progression or stability in primary open-angle glaucoma. Am J Ophthalmol 2000;130:274-9.

48. Yamazaki Y, Drance SM. The relationship between progression of visual field defects and retrobulbar circulation in patients with glaucoma. Am J Ophthalmol 1997;124:287-95.

49. Yamazaki Y, Hayamizu F, Tanaka C. Effects of long-term methylcobalamin treatment on the progression of visual field defects in normal-tension glaucoma. Curr Ther Res Clin Exp 2000;61:443-51.

50. Zahari M, Mukesh BN, Rait JL, Taylor HR, McCarty CA. Progression of visual field loss in open angle glaucoma in the Melbourne Visual Impairment Project. Clinical and Experimental Ophthalmology 2006;34:20-6.

51. Zeimer RC, Wilensky JT, Gieser DK, Viana MA. Association between intraocular pressure peaks and progression of visual field loss. Ophthalmology 1991;98:64-9.

52. Blumenthal EZ, Sample PA, Zangwill L, Lee AC, Kono Y, Weinreb RN. Comparison of long-term variability for standard and short-wavelength automated perimetry in stable glaucoma patients. Am J Ophthalmol 2000;129:309-13.

53. Streiner DL, Norman GR. Health measurement scales: a practical guide to their development and use. In, 4th ed. Oxford: Oxford University Press, 2008b.

54. Coleman AL, Miglior S. Risk factors for glaucoma onset and progression. Surv Ophthalmol 2008;53(suppl 1):3-10.

55. Artes PH, Hutchison DM, Nicolela MT, LeBlanc RP, Chauhan BC. Threshold and variability properties of matrix frequency-doubling technology and standard automated perimetry in glaucoma. Invest Ophthalmol Vis Sci 2005;46:2451-7.

56. Artes PH, Iwase A, Ohno Y, Kitazawa Y, Chauhan BC. Properties of perimetric threshold estimates from Full Threshold, SITA Standard, and SITA Fast strategies. Invest Ophthalmol Vis Sci 2002;43:2654-9.

57. Bengtsson B. Reliability of computerized perimetric threshold tests as assessed by reliability indices and threshold reproducibility in patients with suspect and manifest glaucoma. Acta Ophthalmol Scand 2000;78:519-22.

58. Bengtsson B, Heijl A. SITA Fast, a new rapid perimetric threshold test. Description of methods and evaluation in patients with manifest and suspect glaucoma. Acta Ophthalmol Scand 1998b;76:431-7.

59. Bengtsson B, Heijl A. Evaluation of a new perimetric threshold strategy, SITA, in patients with manifest and suspect glaucoma. Acta Ophthalmol Scand 1998a;76:268-72.

60. Bjerre A, Grigg JR, Parry NR, Henson DB. Test-retest variability of multifocal visual evoked potential and SITA standard perimetry in glaucoma. Invest Ophthalmol Vis Sci 2004;45:4035-40.

61. Blumenthal EZ, Sample PA, Berry CC, Lee AC, Girkin CA, Zangwill L, et al. Evaluating several sources of variability for standard and SWAP visual fields in glaucoma patients, suspects, and normals. Ophthalmology 2003;110:1895-902.

62. Chauhan BC, Johnson CA. Test-retest variability of frequency-doubling perimetry and conventional perimetry in glaucoma patients and normal subjects. Invest Ophthalmol Vis Sci 1999;40:648-56. 
63. Fitzke FW, Crabb DP, McNaught Al, Edgar DF, Hitchings RA. Image processing of computerised visual field data. Br J Ophthalmol 1995;79:207-12.

64. Jampel HD, Vitale S, Ding Y, Quigley H, Friedman D, Congdon N, et al. Test-retest variability in structural and functional parameters of glaucoma damage in the glaucoma imaging longitudinal study. J Glaucoma 2006;15:152-7.

65. Katz J, Quigley HA, Sommer A. Repeatability of the Glaucoma Hemifield Test in automated perimetry. Invest Ophthalmol Vis Sci 1995;36:1658-64.

66. McMillan TA, Stewart WC, Hunt HH. Association of reliability with reproducibility of the glaucomatous visual field. Acta Ophthalmol (Copenh) 1992;70:665-70.

67. Schiefer U, Pascual JP, Edmunds B, Feudner E, Hoffmann EM, Johnson CA, et al. Comparison of the new perimetric GATE strategy with conventional full-threshold and SITA standard strategies. Invest Ophthalmol Vis Sci 2009;50:488-94.

68. Sekhar GC, Naduvilath TJ, Lakkai M, Jayakumar AJ, Pandi GT, Mandal AK, et al. Sensitivity of Swedish interactive threshold algorithm compared with standard full threshold algorithm in Humphrey visual field testing. Ophthalmology 2000;107:1303-8.

69. Spry PG, Henson DB, Sparrow JM, North RV. Quantitative comparison of static perimetric strategies in early glaucoma: test-retest variability. J Glaucoma 2000;9:247-53.

70. Spry PG, Johnson CA, McKendrick AM, Turpin A. Variability components of standard automated perimetry and frequency-doubling technology perimetry. Invest Ophthalmol Vis Sci 2001;42:1404-10.

71. Spry PG, Johnson CA, McKendrick AM, Turpin A. Measurement error of visual field tests in glaucoma. Br J Ophthalmol 2003;87:107-12.

72. Tattersall CL, Vernon SA, Menon GJ. Mean deviation fluctuation in eyes with stable Humphrey 24-2 visual fields. Eye 2007;21:362-6.

73. Wall M, Woodward KR, Doyle CK, Artes PH. Repeatability of automated perimetry: a comparison between standard automated perimetry with stimulus size III and V, matrix, and motion perimetry. Invest Ophthalmol Vis Sci 2009;50:974-9.

74. Wyatt HJ, Dul MW, Swanson WH. Variability of visual field measurements is correlated with the gradient of visual sensitivity. Vision Res 2007;47:925-36.

75. The AGIS Investigators. The Advanced Glaucoma Intervention Study (AGIS): 7. The relationship between control of intraocular pressure and visual field deterioration. Am J Ophthalmol 2000;130:429-40. 



\section{CHAPTER 6}

\section{A new strategy to identify the best method for assessing glaucomatous visual field progression}

Authors:

Paul J.G. Ernest

Jan S.A.G. Schouten

Henny J.M. Beckers

Martin H. Prins

Carroll A.B. Webers

Submitted 


\section{ABSTRACT}

Purpose: To present a new strategy to validate different methods to assess visual field progression using associations with known prognostic factors.

Methods: We included 177 consecutive open-angle glaucoma patients from 2001 to 2003. Follow-up visual fields were collected from the Humphrey Field Analyzer until 2010. Visual field progression was assessed with ten predefined methods and by three assessors. Three baseline prognostic factors (i.e. intraocular pressure (IOP), visual field mean deviation value, and age) and two follow-up prognostic factors (i.e. mean IOP and long-term IOP fluctuation) were considered. The strength of the association between the combination of these five factors and visual field progression according to each method was investigated by comparing the extent to which progressive and nonprogressive patients could be discriminated by the multivariable prognostic model, expressed as the area under the receiver operating characteristic curve (AUC).

Results: On average, 7.8 visual fields were performed within a follow-up time of 7.3 years. The mean incidence of visual field progression for the different methods was $33.5 \%$, ranging from $12.4 \%$ to $59.3 \%$. AUC values ranged from 0.57 to 0.72 . Overall, three methods had higher AUC values compared with the other methods: assessment of visual fields by assessor C, the Advanced Glaucoma Intervention Study method, and pointwise linear regression analysis using total deviation values.

Conclusions: The ability to discriminate progressive from nonprogressive patients with prognostic factors is variable, depending on the method and cut-off points used to assess glaucomatous visual field progression. A high discriminative ability confirms the validity of a method. 


\section{Introduction}

Open-angle glaucoma ( $\mathrm{OAG}$ ) is characterized by visual field progression over months to years. ${ }^{1}$ The general goal of glaucoma management is to maintain the patient's visual function and related quality of life. ${ }^{2}$ Automated visual field testing is regularly repeated to differentiate rapidly progressing patients from slowly progressing patients as a means to direct more intensive treatment to those with a high risk of becoming visually impaired. A large number of methods to assess visual field progression have been developed. ${ }^{3}$ Due to the lack of a gold standard, the relative merits of these methods are currently unclear.

Even in the absence of a gold standard, however, diagnostic tests can still be compared by following a different strategy. ${ }^{4,5}$ For example, the relation between a certain definition of glaucomatous visual field progression and intraocular pressure (IOP) can be investigated. High IOP is among the most consistent prognostic factors for progression of glaucoma and is one of the few factors that can be modified by medical or surgical treatment to slow down progression. ${ }^{6-8}$ Therefore, a method that is assumed to assess glaucomatous visual field progression should find a relation between progression and IOP. When No relation between IOP and glaucomatous progression is found, one should question the validity of the assessment method. In clinimetrics, this type of validation is termed "construct validation" and the relation between IOP and progression is termed a "construct". 9 . We defined three additional constructs for this study: more visual field progression should be associated with more IOP variation, with more advanced visual field loss at baseline, and with higher age. In ophthalmology, the concept of construct validity has only been used for the validation of patient questionnaires and training tools for ophthalmic surgery. ${ }^{10,11}$ However, it has not been applied to validate methods to assess visual field progression.

In this study, we present a new strategy to identify the best method for assessing glaucomatous visual field progression by comparing the extent to which progressive and nonprogressive patients can be discriminated by a multivariable prognostic model containing well-known prognostic factors for glaucomatous progression.

\section{Methods}

\section{Patient selection}

We used the database of the DUtch Research project on treatment outcome IN Glaucoma patients (DURING) study to identify patients who were consecutively recruited in 
the latter project from 2001 to $2003 .{ }^{12,13}$ All patients were treated with topical glaucoma medication at baseline. For the current study we collected information of OAG patients from five out of the nine originally participating Dutch hospitals, including one academic hospital. There were 185 patients who were diagnosed by a participating ophthalmologist as having OAG at the baseline visit of the DURING study and had confirmed visual field loss. The baseline Humphrey Field Analyzer (HFA) (Carl Zeiss Meditec, Dublin, CA, USA) 24-2 or 30-2 visual field should have a Glaucoma Hemifield Test (GHT) "outside normal limits" and/or a significant $(p<0.05)$ deviation of the pattern standard deviation (PSD) index. At least six reliable Swedish Interactive Thresholding Algorithm (SITA) Fast or SITA Standard visual field tests with $<15 \%$ false positive answers, $<15 \%$ false negative answers and $<20 \%$ fixation losses were available for each patient, excluding the first visual field to reduce possible learning effects. We checked all patient records to rule out other ocular comorbidity that could have caused visual field deterioration during the follow-up period and excluded eight patients for this reason. Therefore, 177 patients were eligible for inclusion in this study.

We selected the most severely affected eye based on the GHT and PSD criteria described before. If both eyes were equally affected one eye from each patient was randomly chosen. All available visual fields were collected until 2010.

This study was approved by the local ethics committees and conformed to the tenets of the Declaration of Helsinki. All patients gave their informed consent prior to inclusion.

\section{Methods to assess visual field progression}

Visual field progression was assessed with ten predefined methods and by three assessors. An overview of these methods is shown in Table 1 . These ten methods were chosen because they have been frequently studied in the literature and were included in our previous meta-analysis about the incidence of progression according to different methods to assess progression. ${ }^{14}$

For the fist two methods, we calculated the rate of progression according to frequently used visual field indices from the HFA: the mean deviation (MD) and the visual field index (VFI). Both indices are expressed as a mean value of individual test points in one visual field, but the VFI is more heavily weighted to central visual field areas. ${ }^{15}$ For each patient, we performed a linear regression analysis of the MD and VFI against time. We used a significant deterioration of the visual field as a definition for visual field progression $(p<0.05)$. In an additional analysis, we added "rate of progression" as criterion for visual field progression and varied its cut-off point. 
The Nonparametric Progression Analysis (NPA) is based on nonparametric ranking of MD values. ${ }^{16}$ This method objectifies the commonly practised method of monitoring glaucoma patients with the use of MD values. A visual field series is considered progressive as the MD value of a follow-up visual field is worse than the MD of the worst of two baseline fields. This has to be confirmed on at least two consecutive visual fields and has to be consistent until the end of the follow-up period. ${ }^{16}$ 
Table 1: Overview of methods used to assess glaucomatous visual field progression

\begin{tabular}{|c|c|c|}
\hline Method & Description of the method & $\begin{array}{l}\text { Incidence of } \\
\text { progression }\end{array}$ \\
\hline AGIS & AGIS-method & $22(12.4 \%)$ \\
\hline CIGTS & CIGTS-method & $26(14.7 \%)$ \\
\hline PLR PD & $\begin{array}{l}\text { Two-omitting PLR with pattern deviation values, using criteria of }<-1 \mathrm{~dB} \text { per year } \\
\text { and } p<0.05 \text { and a cut-off point of } \geq 3 \text { locations at } 3 \text { consecutive examinations }\end{array}$ & $26(14.7 \%)$ \\
\hline & $\begin{array}{l}\text { At least one of these scotoma criteria: deepening of } \geq 2 \text { points } \geq 10 \mathrm{~dB} \text { in the same } \\
\text { locations; expansion of } \geq 2 \text { points } \geq 10 \mathrm{~dB} \text {; development of } \geq 2 \text { adjacent points, not }\end{array}$ & \\
\hline Boden & $\begin{array}{l}\text { within or adjacent to a baseline scotoma, with } p<1 \% \text { or worse at pattern deviation } \\
\text { plot or } 1 \text { previously normal location in central } 10 \text { degrees } \geq 10 \mathrm{~dB} \text {. Confirmations at } \\
\text { the same locations on } 3 \text { consecutive fields, based on pattern deviation values }\end{array}$ & $38(21.5 \%)$ \\
\hline GPA & Glaucoma Progression Analysis & $35(22.5 \%)^{*}$ \\
\hline PLR TD & $\begin{array}{l}\text { Two-omitting PLR with total deviation values, using criteria of }<-1 \mathrm{~dB} \text { per year and } \\
\mathrm{p}<0.05 \text { and a cut-off point of } \geq 3 \text { locations at } 3 \text { consecutive examinations }\end{array}$ & $42(23.7 \%)$ \\
\hline Subj $_{\mathrm{A}}$ & Blinded subjective assessment of Humphrey overview printouts by assessor A & $65(36.7 \%)$ \\
\hline Subjc & Blinded subjective assessment of Humphrey overview printouts by assessor C & $70(39.5 \%)$ \\
\hline Subj & $\begin{array}{l}\text { Likely progression based on the subjective assessment of Humphrey overview } \\
\text { printouts by observers who were blinded for other clinical information and did not } \\
\text { use a predefined criterion of progression; two out of three observers had to assign } \\
\text { a series as progressive }\end{array}$ & $74(41.8 \%)$ \\
\hline VFI & Linear regression analysis of the $\mathrm{VFI}$, using a criterion of $p<0.05$ & $83(46.9 \%)$ \\
\hline MD & Linear regression analysis of the $\mathrm{MD}$, using a criterion of $\mathrm{p}<0.05$ & $85(48.0 \%)$ \\
\hline NPA & Nonparametric progression analysis based on nonparametric ranking of MD values & $96(54.2 \%)$ \\
\hline Subj $_{B}$ & Blinded subjective assessment of Humphrey overview printouts by assessor B & $105(59.3 \%)$ \\
\hline
\end{tabular}

The ten methods to assess progression and the three assessors are ranked according to the incidence of visual field progression as shown in the third column.

*Excluding 22 eyes that had no possibility to fulfill the progression criteria according to the Glaucoma Progression Analysis.

AGIS = Advanced Glaucoma Intervention Study, CIGTS = Collaborative Initial Glaucoma Treatment Study, PLR $P D=$ pointwise linear regression analysis based on pattern deviation values, Boden = method according to Boden et al., GPA = Glaucoma Progression Analysis, PLR TD = pointwise linear regression analysis based on total deviation values, $\operatorname{Subj}_{\mathrm{A}}$ to $\mathrm{Subj}_{\mathrm{C}}=$ subjective method according to assessor $\mathrm{A}$ to $\mathrm{C}$, Subj = subjective method according to the three assessors, VFI = visual field index, MD = mean deviation, NPA = nonparametric progression analysis, $\mathrm{dB}=$ decibels.

The fourth method is the Glaucoma Progression Analysis (GPA), which is similar to the visual field endpoint in the Early Manifest Glaucoma Trial (EMGT). ${ }^{17}$ The GPA is an event analysis based on pattern deviation values and is included in the new HFA software. 
When significant deterioration $(p<0.05)$ is seen on the pattern deviation probability maps of the GPA printouts in the same three or more points on three consecutive follow-up tests, the software interprets this as likely progression. ${ }^{17}$ We performed the analyses after exclusion of eyes that had no possibility to fulfil GPA progression criteria due to exclusion of visual fields with advanced loss or different perimetric strategies.

We performed two pointwise linear regression analyses (PLR), one using total and the other using pattern deviation values of individual test points (i.e. PLR TD and PLR PD respectively). ${ }^{18}$ Individual total deviation values were derived by conversion of serial HFA output with the MSDOS version of PeriData (PeriData Software GmbH, Huerth, Germany). Total deviation values in PeriData are slightly different from HFA values due to different reference groups used for calculation of these values. We also calculated pattern deviation values for each test point by subtracting the total deviation value of the $7^{\text {th }}$ best point of the 24-2 total deviation visual field from the total deviation values of all other points. ${ }^{19}$ We performed a linear regression analysis of the total deviation and pattern deviation values of each individual test location of the 24-2 visual field.

The Advanced Glaucoma Intervention Study (AGIS) method and the Collaborative Initial Glaucoma Treatment Study (CIGTS) method are both based on the AGIS study. ${ }^{20}$ Both the AGIS method and the CIGTS method use a scoring system to grade each visual field in the follow-up period. The AGIS score is based on the actual decibel deviations at the total deviation plot of the 24-2 visual field, while the CIGTS algorithm is based on the $\mathrm{p}$ values obtained from the total deviation probability plot. Both scoring systems range from 0 to 20, with 0 representing no field loss and 20 end-stage disease. Visual field series are considered to be progressive if the score has increased by four or more from the baseline reference field (with the AGIS method) or three or more compared with the average score of two baseline fields (with the CIGTS method). With both methods, progression has to be confirmed by two additional tests. ${ }^{21}$ We calculated the CIGTS score for each visual field on the printouts of total deviation probability plot and we calculated the AGIS score using the total deviation values derived with PeriData.

We also performed a blinded subjective assessment of the series of visual fields (i.e. method Subj). Three authors (CAW, HJB, JSS), who are ophthalmologists including two glaucoma specialists, independently judged if a visual field series was not progressive, possibly progressive, probably progressive, or definitely progressive due to glaucoma. The assessors used overview printouts of the HFA and were blinded for the patient's name and other clinical information. They received no instructions for analyzing the series of visual fields to give a good representation of subjective assessment in daily clinical practice. For defining a visual field series as progressive, at least two assessors had to assign a series as definitely or probably progressive. 
Finally, the method of Boden and co-workers is a progression algorithm based on the clinical judgement of scotomas. ${ }^{22}$ The development, expansion, and the deepening of scotomas is determined with use of pattern deviation values and is also based on the 24-2 visual field. ${ }^{22}$ Pattern deviation values were derived with PeriData.

\section{Prognostic factors for progression}

We used the following three baseline prognostic factors for validating the methods: baseline MD value, baseline age, and baseline IOP. ${ }^{6}$ IOP values were derived from case records. We defined baseline IOP as the mean of the last two IOPs up to and including the baseline visit. We categorized baseline MD value in tertiles, because earlier studies have shown that the relation between visual field progression and baseline visual field loss is not uniform across the entire range of visual field loss. Progression is positively related with baseline visual field severity in early disease stages and negatively related

with visual field severity in advanced disease stages. ${ }^{23-25}$ The group with the best visual field was selected as reference group for the groups with moderate visual field loss (i.e. $M D$ values $\leq-4.0$ decibels $(\mathrm{dB})$ and $>-10.2 \mathrm{~dB}$ ) and severe visual field loss (i.e. MD values $\leq-10.2 \mathrm{~dB})$.

We also used two prognostic factors that were collected during the follow-up period: mean IOP and long-term IOP fluctuation. ${ }^{26,27}$ We used follow-up IOP values from the baseline visit to the date of the last visual field. IOP values within one month after filtration or cataract surgery were excluded from the analysis. In order to control for the different time intervals between follow-up visits, we calculated a weighted mean IOP according to Harju. ${ }^{28}$ IOP fluctuation was defined as the standard deviation of follow-up IOP values.

\section{Statistical analyses}

First, we calculated the incidence of glaucomatous visual field progression in our population during the follow-up period according to each method. Kappa $(\kappa)$ statistics were calculated to assess agreement between the ten methods and between the subjective assessments of the three assessors.

We validated the methods with a prognostic model by performing a multivariable logistic regression analysis for each method. We used the binary outcome of the method to assess visual field progression as the dependent variable and the five prognostic factors as the independent variables in the analysis. With the predicted probabilities derived from the logistic regression analysis, we created receiver operating characteristic (ROC) curves to assess the association of each method with the combination of factors. We calculated the area under the ROC curve (AUC) for comparing the associa- 
tions found with the different methods. An AUC near 0.5 indicates that there is no association between the method and the prognostic model and a value of 1.0 indicates a perfect association. ${ }^{29}$ Thus, the method with the highest area under the ROC curve was assumed to be a more valid method to assess visual field progression. The individual contribution of the five factors in the multivariable model was also assessed by the odds ratio and the accompanying statistical significance.

In an additional analysis, we investigated the influence of "rate of progression" as an extra criterion for visual field progression according to the MD and VFI methods that were solely based on the statistical significance of progression. We used a rate of progression <-1 dB per year for the MD method and <-3\% per year for the VFI method as cut-off points. To investigate the influence of different cut-off points on AUC values, we also varied this "rate of progression" criterion for both methods.

\section{Results}

Descriptive statistics of the study population are summarized in Table 2 . The mean baseline MD value was $-8.2 \mathrm{~dB}$ and the mean age at baseline was 65 years. A mean of 7.8 reliable visual fields were performed during a mean follow-up time of 7.3 years.

\section{Visual field progression}

The mean MD rate of progression was $-0.46 \mathrm{~dB}$ per year. Table 1 shows the incidence of visual field progression according to the ten different methods to assess progression and for each of the three assessors. The mean incidence of progression was $33.5 \%$ (standard deviation (SD) \pm 16.1 ), which ranged from $12.4 \%$ for the AGIS method to $59.3 \%$ for the subjective assessment of visual fields by assessor $\mathrm{B}$.

For the GPA method, several visual field examinations could not be used either because of a different test strategy compared with the rest of the series of examinations $(n=49)$ or a too severely depressed visual field as indicated by the HFA software $(n=115)$. Therefore, 22 patients had no possibility to fulfill the progression criteria for the GPA. We analyzed the GPA strategy without these 22 patients.

The mean $k$ value of the ten methods was 0.35 (SD \pm 0.15 ), ranging from 0.74 for the agreement between the NPA and MD method to 0.15 for the NPA and AGIS method. The interobserver agreement between the three ophthalmologists, expressed as $k$ values, was 0.44 for assessor $A$ versus $B, 0.58$ for assessor $A$ versus $C, 0.47$ for assessor $B$ versus $C$. 


\section{Prognostic factors}

For each method, the coefficients and statistical significance of the five prognostic factors in the multivariable analysis are shown in Table 3. The most consistently related prognostic factors with progression were IOP fluctuation and age, with six and four statistically significant $(p<0.05)$ associations respectively. A larger IOP fluctuation and a higher age resulted in more progression. A higher baseline IOP also consistently resulted in more progression, but only one method resulted in a statistically significant association in the multivariable analysis. More advanced baseline visual field loss resulted in more progression for all methods except the AGIS method and the GPA method. With three positive and ten negative associations and a lack of statistical significance for all methods, the relation of mean IOP with progression was the least consistent among the different methods (Table 3).

\section{Validation of methods}

The results of the logistic regression analyses are shown in the last column of Table 3. The mean AUC of the ten methods and the three assessors was 0.66 (SD \pm 0.04 ). AUC ranged from 0.57 (confidence interval $(\mathrm{Cl}) 0.46,0.67$ ) for the GPA method to $0.72(\mathrm{Cl}$ $0.64,0.80)$ for assessor $C$. Overall, three methods had higher AUC values compared with the other methods: assessor C, AGIS, and PLR TD. Two methods had a weaker association with prognostic factors compared to the other methods: the MD and GPA. All other methods had AUC values around 0.66 (Table 3).

When we added <-1 dB per year for the MD method and <-3\% per year for the VFI method as progression criteria, the AUC improved from 0.61 to $0.77(\mathrm{Cl} 0.66,0.88)$ and from 0.65 to $0.77(\mathrm{Cl} 0.65,0.88)$ respectively. Figure 1 shows the ROC curve for the MD method and ROC curves for the MD method with the addition of different criteria for the rate of progression. The AUC for the MD method increased when stricter criteria for progression were used. For the $<-0.5 \mathrm{~dB},<-1.5 \mathrm{~dB}$, and $<-2 \mathrm{~dB}$ per year criteria, the

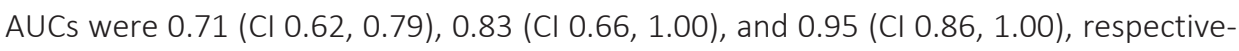
ly. We found analogous results for the VFI method. 
Table 2: Descriptive statistics of the study population

Descriptive statistics

Follow-up period

Number of visual fields

Clinical centre
Values in the study population $(n=177)$

7.3 years $( \pm 1.2)$

$7.8( \pm 1.9)$

$109(61.5 \%)$

$16(9 \%)$

$14(8 \%)$

$16(9 \%)$

$22(12.5 \%)$

75 (42\%)

Female sex

Diagnosis

$\begin{array}{rc}\text { POAG } & 141(79.5 \%) \\ \text { NTG } & 28(16 \%) \\ \text { PG } & 5(3 \%) \\ \text { PXG } & 3(1.5 \%)\end{array}$

Cataract surgery during follow-up

$29(16 \%)$

Filtration surgery during follow-up $13(7 \%)$

Prognostic Factors in the Study Eye

Baseline age

64.8 years $( \pm 9.5)$

Baseline IOP

$15.5 \mathrm{mmHg}( \pm 3.4)$

Baseline visual field MD

$$
-8.2 \mathrm{~dB}( \pm 6.5)
$$

$\mathrm{MD} \leq-4.0 \mathrm{~dB}$ and $>-10.2 \mathrm{~dB}$

$M D \leq-10.2 d B$

$59(33.3 \%)$

$14.7( \pm 2.8)$

$2.4( \pm 1.1)$

Follow-up IOP standard deviation

Visual field progression

Observed MD rate in the study eye

$-0.46 \mathrm{~dB} /$ year $( \pm 0.59)$

Values are displayed as means and standard deviations or frequencies and percentages.

POAG = primary open-angle glaucoma, $\mathrm{NTG}=$ normal-tension glaucoma, $\mathrm{PG}=$ pigment glaucoma, $\mathrm{PXG}=$ pseudoexfoliation glaucoma, $\mathrm{IOP}=$ intraocular pressure, $\mathrm{MD}=$ mean deviation, $\mathrm{dB}=$ decibels. 


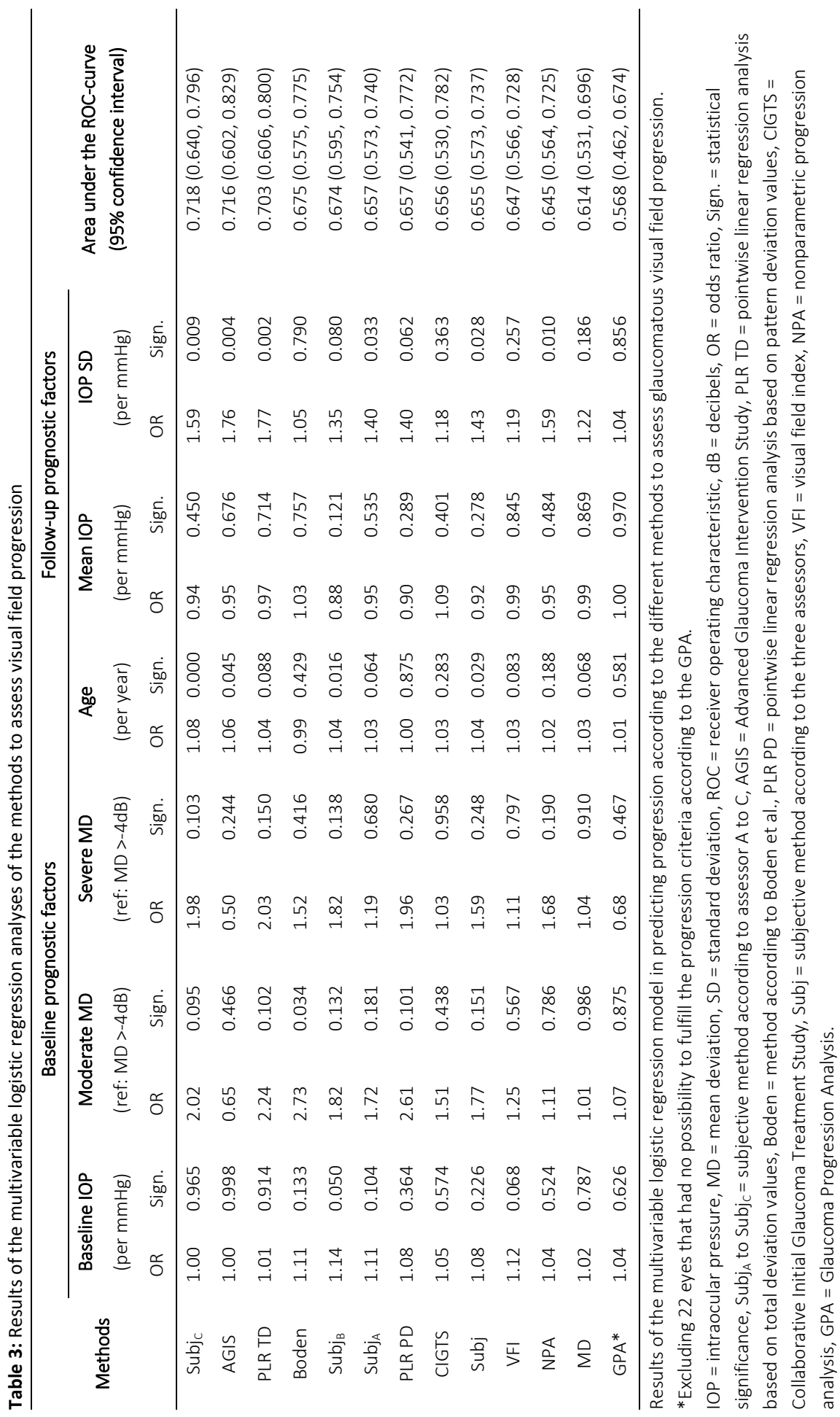




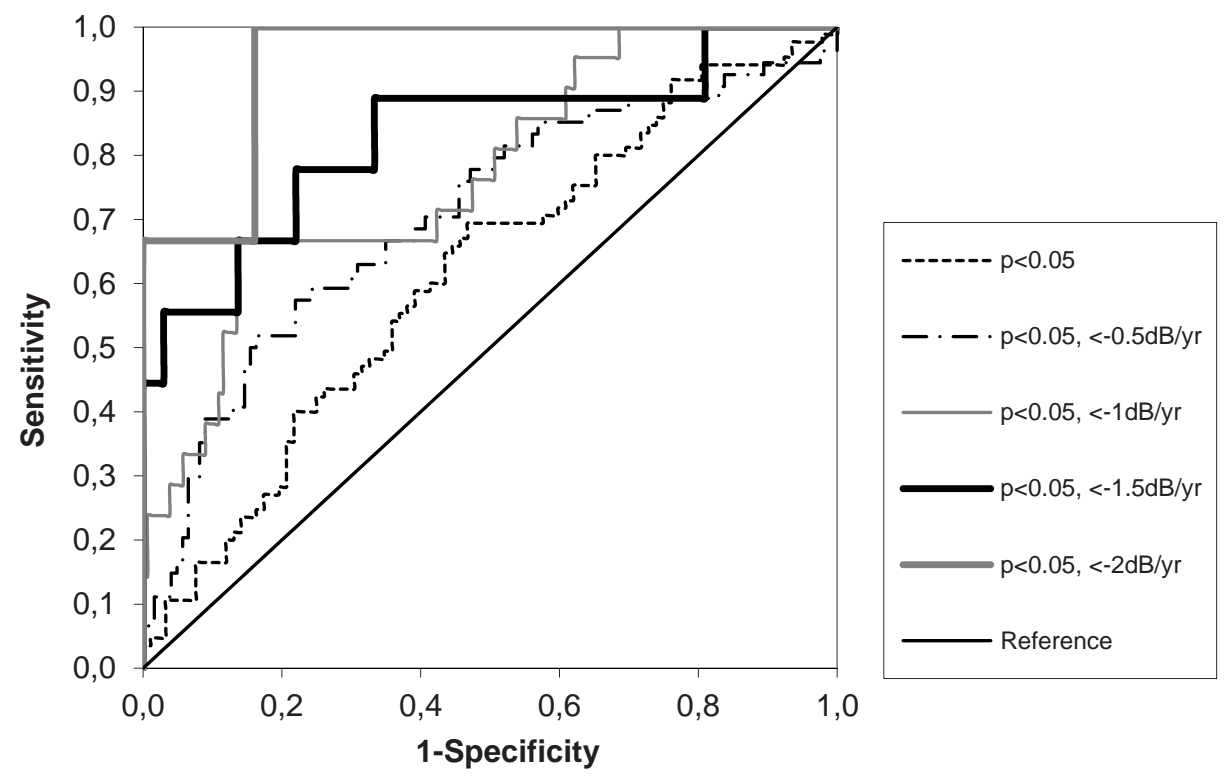

Figure 1

Receiver operating characteristic curves for different cut-off points of the mean deviation (MD) method. The area under the receiver operating characteristic curve (AUC) increases with stricter rate of progression criteria for the MD. The AUC ranges from 0.61 for the MD method with statistical significance $(p<0.05)$ as criterion, to 0.95 when adding the stricter criterion of a rate of progression <-2dB per year alongside the statistical significance criterion.

$\mathrm{dB} /$ year $=$ decibels per year.

\section{Discussion}

In this study, the following methods to assess glaucomatous visual field progression had a relatively high ability to discriminate progressive and nonprogressive patients with well-known prognostic factors: assessor C, the AGIS method, and PLR using total deviation values. The results of the current validation study are an argument to prefer one of these methods. However, validation of methods is a continuous process, should include comparisons with other variables, and should be performed in different populations. ${ }^{9}$ Ultimately, comparisons should include patient-centered outcome measures, like quality-of-life. For example, one study performed a validation of the change in MD values over four years and related it with the minimal clinically important difference in vision-specific health-related quality of life. ${ }^{30}$ 
In the current study, direct comparisons between methods to assess glaucomatous visual field progression show that there is a high variability in the incidence of progression between methods. This variability is comparable with our recent network metaanalysis that ranked methods according to their incidence of progression based on direct and indirect comparisons. ${ }^{14}$ The present ranking of incidences largely correspond with the ranking in the meta-analysis, although MD and VFI methods had relatively higher incidences and PLR TD and PLR PD methods had relatively lower incidences. ${ }^{14}$ This may be explained by the shorter follow-up time of the two studies that studied the MD and VFI methods in the meta-analysis (i.e. 3.3 and 5.1 years) and the higher number of visual fields of the study that studied the PLR methods (i.e. 21 visual fields), for which was only partially adjusted. ${ }^{14,18,31,32}$ The incidence of progression with the GPA in the current study was also higher, mainly because we analyzed the GPA strategy without 22 patients who had no possibility to fulfill the progression criteria for the GPA. The moderate agreement we found between methods and between different ophthalmologists has also been previously described. ${ }^{33-35}$

It has been shown earlier that the relation between a prognostic factor and visual field progression is dependent on the method used to assess visual field progression and the statistical analysis that is used. ${ }^{36}$ We applied the same statistical analysis, but varied the method to assess visual field progression. We found a large variability in the coefficients and statistical significance of the tested prognostic factors, depending on the method used to assess progression. No prognostic factor was tested statistically significant for all methods. Mean IOP was the prognostic factor that had the weakest relation with visual field progression in our study. The reason for this may be that treatment in clinical practice is mainly guided by IOP values. In an observational study, the effect of changing treatment based on IOP could dilute and even reverse the relation between IOP and visual field progression. IOP fluctuation was the strongest prognostic factor, which is in line with recent evidence from the Collaborative Initial Glaucoma Treatment Study. ${ }^{37}$

Unlike more complicated methods, the MD and VFI index are often used for visual field assessment in clinical practice. For example, all three assessors have indicated that they used the MD index and two of them used the VFI for their blinded subjective assessment of visual field series in this study. Interestingly, we found that progressive and nonprogressive patients according to the MD and VFI methods were poorly discriminated by the multivariable prognostic model. This may be explained by the definition of both methods, which only included statistical significance of the progression. Besides the severity of visual field progression, statistical significance is also dependent on the number of visual fields available. This implies that even a low rate of visual field pro- 
gression could be statistically significant when many visual fields are included in the assessment. Therefore, we repeated the analysis after addition of "rate of progression" as criterion for visual field progression. A combination of statistical and rate criteria for the MD and VFI methods resulted in the highest discriminative ability of all methods in this study. Moreover, when stricter criteria for visual field progression were used for the MD and VFI methods, stronger associations were found with the combination of prognostic factors. Stricter progression criteria assign progression to a smaller group of patients who have more characteristics of typical progressive patients. Therefore, applying stricter progression criteria enhances the contrast in prognostic factors between the progressive and nonprogressive groups which is shown by the larger AUC values. It also shows that a more valid method to assess progression may not be only achieved by choosing a different method but also by considering a different cut-off point. This is akin to clinical practice were a patient with More progression on a scale is more likely to be considered to be really progressing.

In conclusion, the relation between glaucomatous visual field progression and prognostic factors is dependent on the method used to assess progression. No prognostic factor was tested statistically significant for all methods. The other way round, the ability of a progression method to discriminate progressive from nonprogressive patients with a combination of well-known prognostic factors is variable. This can be used to validate methods to assess progression, where a high discriminative ability confirms the validity of a method. 


\section{References}

1. Heijl A, Bengtsson B, Hyman L, Leske MC. Natural history of open-angle glaucoma. Ophthalmology 2009;116:2271-6.

2. European Glaucoma Society. Terminology and Guidelines for Glaucoma (3rd ed.). Savona, Italy: Dogma, 2008.

3. Ernest PJ, Schouten JS, Beckers HJ, Hendrikse F, Prins MH, Webers CA. The evidence base to select a method for assessing glaucomatous visual field progression. Acta Ophthalmol 2012;90:101-8.

4. Reitsma JB, Rutjes AW, Khan KS, Coomarasamy A, Bossuyt PM. A review of solutions for diagnostic accuracy studies with an imperfect or missing reference standard. J Clin Epidemiol 2009;62:797-806.

5. Bland JM, Altman DG. Statistics Notes: Validating scales and indexes. BMJ 2002;324:606-7.

6. Ernest PJ, Schouten JS, Beckers HJ, Hendrikse F, Prins MH, Webers CA. An Evidence-Based Review of Prognostic Factors for Glaucomatous Visual Field Progression. Ophthalmology 2013;120:512-9.

7. Bahrami H. Causal inference in primary open angle glaucoma: specific discussion on intraocular pressure. Ophthalmic Epidemiol 2006;13:283-9.

8. Heijl A, Leske MC, Bengtsson B, Hyman L, Bengtsson B, Hussein M. Reduction of intraocular pressure and glaucoma progression: results from the Early Manifest Glaucoma Trial. Archives of Ophthalmology 2002;120:1268-79.

9. Streiner DL, Norman GR. Health measurement scales: a practical guide to their development and use. Oxford: Oxford University Press; 2008:257-258.

10. Privett B, Greenlee E, Rogers G, Oetting TA. Construct validity of a surgical simulator as a valid model for capsulorhexis training. J Cataract Refract Surg 2010;36:1835-8.

11. de Boer MR, Moll AC, de Vet HC, Terwee CB, Volker Dieben HJ, van Rens GH. Psychometric properties of vision-related quality of life questionnaires: a systematic review. Ophthalmic Physiol Opt 2004;24:257-73.

12. van der Valk R, Schouten JS, Webers CA, Hendrikse F, Prins MH. Changes in glaucoma treatment and achieved IOP after introduction of new glaucoma medication. Graefes Arch Clin Exp Ophthalmol 2006;244:1267-72.

13. van der Valk R, Webers CA, Hendrikse F, de Vogel SC, Prins MH, Schouten JS. Predicting intraocular pressure change before initiating therapy: timolol versus latanoprost. Acta Ophthalmologica Scandinavica 2008;86:415-8.

14. Ernest PJ, Viechtbauer W, Schouten JS, Beckers HJ, Hendrikse F, Prins MH, et al. The influence of the assessment method on the incidence of visual field progression in glaucoma: a network meta-analysis. Acta Ophthalmol 2012;90:10-9.

15. Bengtsson B, Heijl A. A visual field index for calculation of glaucoma rate of progression. American Journal of Ophthalmology 2008;145:343-53.

16. Wesselink C, Heeg GP, Jansonius NM. Glaucoma monitoring in a clinical setting: Glaucoma progression analysis vs nonparametric progression analysis in the Groningen longitudinal glaucoma study. Archives of Ophthalmology 2009;127:270-4.

17. Leske MC, Heijl A, Hyman L, Bengtsson B. Early Manifest Glaucoma Trial: design and baseline data. Ophthalmology 1999;106:2144-53.

18. Artes PH, Nicolela MT, LeBlanc RP, Chauhan BC. Visual field progression in glaucoma: total versus pattern deviation analyses. Investigative Ophthalmology and Visual Science 2005;46:4600-6.

19. Heijl A, Lindgren G, Olsson J, Asman P. Visual field interpretation with empiric probability maps. Arch Ophthalmol 1989;107:204-8.

20. The AGIS Investigators. Advanced Glaucoma Intervention Study. 2. Visual field test scoring and reliability. Ophthalmology 1994;101:1445-55.

21. Katz J. Scoring systems for measuring progression of visual field loss in clinical trials of glaucoma treatment. Ophthalmology 1999;106:391-5. 
22. Boden C, Blumenthal EZ, Pascual J, McEwan G, Weinreb RN, Medeiros F, et al. Patterns of glaucomatous visual field progression identified by three progression criteria. American Journal of Ophthalmology 2004;138:1029-36.

23. Rao HL, Kumar AU, Babu JG, Senthil S, Garudadri CS. Relationship between Severity of Visual Field Loss at Presentation and Rate of Visual Field Progression in Glaucoma. Ophthalmology 2011;118:249-53.

24. Blackwell B, Gaasterland D, Ederer F, Dally LG, VanVeldhuisen P, Prum BE, et al. The Advanced Glaucoma Intervention Study (AGIS): 12. Baseline risk factors for sustained loss of visual field and visual acuity in patients with advanced glaucoma. American Journal of Ophthalmology 2002;134:499-512.

25. Leske MC, Heijl A, Hussein M, Bengtsson B, Hyman L, Komaroff E. Factors for glaucoma progression and the effect of treatment: The early manifest glaucoma trial. Archives of Ophthalmology 2003;121:48-56.

26. Leske MC, Heijl A, Hyman L, Bengtsson B, Dong L, Yang Z. Predictors of long-term progression in the early manifest glaucoma trial. Ophthalmology 2007;114:1965-72.

27. Nouri Mahdavi K, Hoffman D, Coleman AL, Liu G, Li G, Gaasterland D, et al. Predictive factors for glaucomatous visual field progression in the Advanced Glaucoma Intervention Study. Ophthalmology 2004;111:1627-35.

28. Harju M. Intraocular pressure and progression in exfoliative eyes with ocular hypertension or glaucoma. Acta Ophthalmol Scand 2000;78:699-702.

29. Harrell FE, Jr., Califf RM, Pryor DB, Lee KL, Rosati RA. Evaluating the yield of medical tests. JAMA 1982;247:2543-6.

30. Patino CM, Varma R, Azen SP, Conti DV, Nichol MB, McKean Cowdin R. The impact of change in visual field on health-related quality of life the los angeles latino eye study. Ophthalmology 2011;118:1310-7.

31. Birch MK, Wishart PK, O'Donnell NP. Determining progressive visual field loss in serial Humphrey visual fields. Ophthalmology 1995;102:1227-34; discussion 34-5.

32. Casas Llera P, Rebolleda G, Munoz Negrete FJ, Arnalich Montiel F, Perez Lopez M, Fernandez Buenaga R. Visual field index rate and event-based glaucoma progression analysis: comparison in a glaucoma population. Br J Ophthalmol 2009;93:1576-9.

33. Heijl A, Bengtsson B, Chauhan BC, Lieberman MF, Cunliffe I, Hyman L, et al. A comparison of visual field progression criteria of 3 major glaucoma trials in early manifest glaucoma trial patients. Ophthalmology 2008;115:1557-65.

34. Katz J, Congdon N, Friedman DS. Methodological variations in estimating apparent progressive visual field loss in clinical trials of glaucoma treatment. Archives of Ophthalmology 1999;117:1137-42.

35. Tanna AP, Bandi JR, Budenz DL, Feuer WJ, Feldman RM, Herndon LW, et al. Interobserver agreement and intraobserver reproducibility of the subjective determination of glaucomatous visual field progression. Ophthalmology 2011;118:60-5.

36. Wesselink C, Marcus MW, Jansonius NM. Risk factors for visual field progression in the groningen longitudinal glaucoma study: a comparison of different statistical approaches. J Glaucoma 2012;21:57985.

37. Musch DC, Gillespie BW, Niziol LM, Lichter PR, Varma R. Intraocular pressure control and long-term visual field loss in the Collaborative Initial Glaucoma Treatment Study. Ophthalmology 2011;118:1766-73. 



\section{CHAPTER 7}

Prevalence of end-of-life visual impairment in patients followed for glaucoma

Authors:

Paul J.G. Ernest

Michiel J.W.M. Busch

Carroll A.B. Webers

Henny J.M. Beckers

Fred Hendrikse

Martin H. Prins

Jan S.A.G. Schouten

Acta Ophthalmologica 2013;91:738-743 


\section{ABSTRACT}

Purpose: To assess the prevalence of end-of-life visual impairment in patients followed for glaucoma.

Methods: Data of 122 patients followed for glaucoma who had died between July 2008 and July 2010 and who had visited the ophthalmology outpatient department of a large non-academic Dutch hospital were collected from the medical files. Sixty-one patients had open-angle glaucoma (OAG) and 61 patients were suspect for glaucoma or had ocular hypertension (OHT). Visual impairment was defined as a Mean Deviation value <$15 \mathrm{~dB}$ or a Snellen visual acuity $<0.3(20 / 60)$ of the better eye. We determined the number of patients with visual impairment on the last patient visit before death and investigated its main explanations.

Results: Overall, the mean age at death was 81.8 years after a mean follow-up period of 9.2 years. Seventy-three percent of all patients had their last visit in the year preceding death. In OAG, 16 patients (26\%) had an end-of-life visual impairment. In nine patients $(15 \%)$ this was caused by glaucoma. Eight of them had substantial visual loss at the initial visit. Six (10\%) impaired OAG cases were mainly explained by ocular comorbidity and there was an equal contribution of comorbidity and glaucoma in one case. Five glaucoma suspects or patients with $\mathrm{OHT}(8 \%)$ were visually impaired at death and these were all caused by ocular comorbidity.

Conclusion: The prevalence of end-of-life visual impairment is considerable in OAG patients. Substantial visual loss at baseline is an important contributing factor. In glaucoma suspects or patients with OHT the prevalence is lower and can be attributed to ocular comorbidity. 


\section{Introduction}

The goal of glaucoma management is to maintain visual function and related quality-oflife (QoL) throughout the patient's life. To reach this goal, glaucoma patients should be identified and treated based on the individual risk of visual disability. ${ }^{1}$

Current identification of glaucoma patients is not optimal because a large number of prevalent cases are undiagnosed. ${ }^{2,3}$ Undiagnosed patients could be identified by routine screening of the general population, but population-based screening for openangle glaucoma $(\mathrm{OAG})$ is not considered to be efficient. ${ }^{4}$ Other screening strategies have been proposed, like case finding during regular optician visits. ${ }^{5}$ On the other hand, we know that some glaucoma patients become blind despite timely treatment. ${ }^{6}$ Determination of rate of visual field progression is increasingly being recognized as an important tool to identify patients who need more intensive treatment to slow down disease progression. ${ }^{7}$ The effectiveness of current screening and treatment regimes should ideally be judged on the basis of visual function and QoL at the end of glaucoma patients' life.

The aim of the present study is to evaluate current glaucoma management strategies by assessing the prevalence of end-of-life visual impairment in a large non-academic Dutch hospital. In cases with an end-of-life visual impairment, we determined whether this was mainly explained by glaucoma or ocular comorbidity.

\section{Methods}

\section{Patient selection}

For this study, we performed a retrospective observational case review. We searched for all patients diagnosed with glaucoma, ocular hypertension (OHT), or suspected glaucoma who were seen at the outpatient ophthalmology department of Catharina Hospital Eindhoven between 2003 and 2010. Catharina Hospital Eindhoven is a large non-academic referral hospital with six ophthalmologists. To find eligible patients, we used the Diagnose Behandel Combinatie-system (DBC-system, DBC-Onderhoud, Utrecht, the Netherlands), which has been developed for registration and payment for delivered care in the Netherlands. Subsequently, the Dutch Health Insurance Database (VECOZO, Tilburg, the Netherlands) was used to retrieve patients who had died. The VECOZO database covers more than $99 \%$ of all people who are insured for health services in the Netherlands. Less than $1 \%$ of the total population in the Netherlands is currently uninsured. ${ }^{8}$ In July 2010, we linked the DBC-system with the VECOZO database 
to retrieve all patients who had glaucoma-related problems and were deceased during the past two years. This search resulted in 158 patients (Fig. 1). After studying patient records, 135 patients with confirmed glaucoma-related problems and at least two visits at the outpatient department were found. This study conformed to the tenets of the Declaration of Helsinki and all national laws of the Netherlands, when applicable.

\section{Data collection}

For each patient, we collected data from the first glaucoma-related visit to the last visit at the outpatient department (i.e. the follow-up period). The first glaucoma-related visit was defined as the first visit in which a patient presented with an elevated intraocular pressure (IOP) or other clinical features related to glaucoma.

Visual function was assessed by Snellen visual acuity and visual field tests (Humphrey Field Analyzer (HFA) (Carl Zeiss Meditec, Dublin, CA, USA)). The Mean Deviation (MD) index of a standard 24-2 or 30-2 HFA visual field was used as a measure of the overall visual field status. SITA visual fields as well as Fastpac visual field tests were performed. If not available, screening or 10-2 visual fields were used. Reliable HFA visual fields were defined as visual fields having less than $15 \%$ false positive answers. Visual fields before the year 1996 were performed with Peritest semi-automated static perimetry. Since 2006, optic nerve imaging was performed additionally to visual field testing using scanning laser polarimetry (SLP) (Gdx VCC, Carl Zeiss Meditec, Dublin, CA, USA). We used monocular best corrected Snellen visual acuity measurements in the analyses.

Diagnoses were set retrospectively after studying patient records. All diagnoses were verified by two authors, including one glaucoma specialist (MJWB and PJGE). OAG ( $n=61)$ was diagnosed if there was a glaucomatous visual field defect in one or both eyes. The OAG group consisted of primary open-angle glaucoma (POAG) ( $n=53)$, normaltension glaucoma (NTG) $(n=7)$, and pseudoexfoliation glaucoma (PXG) (n=1). NTG was diagnosed in patients with IOP always below $21 \mathrm{mmHg}$. In six POAG patients, the diagnosis relied on imaging of the optic nerve because of absence of visual fields. Glaucoma suspects $(n=9)$ had normal visual fields but other signs suggestive for glaucoma. OHT ( $n=52$ ) was defined when IOP was $>21 \mathrm{mmHg}$, without damage of the visual field, SLP, or optic disc. Glaucomas associated with, or subsequent to other ocular or systemic disorders $(n=10)$ or where a narrow angle was likely to be responsible for the optic nerve damage ( $n=3)$ were excluded (Fig. 1$)$. 


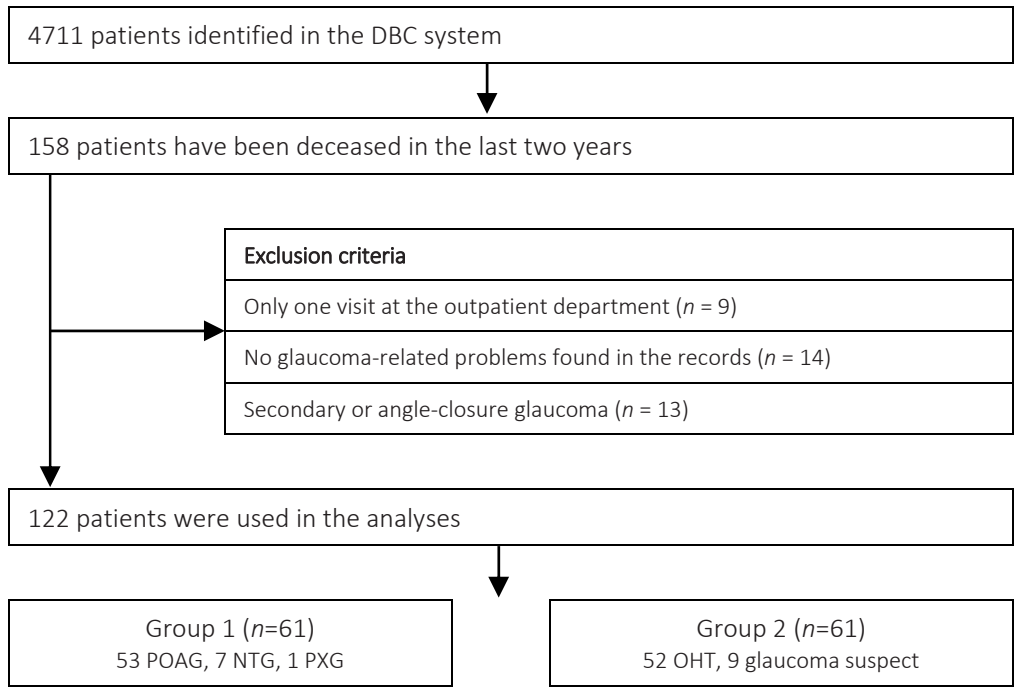

Figure 1 Flow chart of the patient selection process

$\mathrm{DBC}=$ Diagnose Behandel Combinatie, $\mathrm{POAG}=$ primary open-angle glaucoma, NTG = normal-tension glaucoma, PXG = pseudoexfoliation glaucoma, $\mathrm{OHT}=$ ocular hypertension.

\section{Data analysis}

To analyze end-of-life visual function, we used the last available and reliable visual field MD value and the last available Snellen visual acuity for the better eye of each patient. The functional status of the better eye has shown to have the highest correlation with QoL. ${ }^{9,10}$ If data were only available for one eye, this was always the better eye.

We defined visual impairment according to the tenth edition of the International Classification of Diseases (ICD-10) of the World Health Organization (WHO). ${ }^{11}$ We considered patients as being visually impaired when they had binocular moderate visual impairment category 1 in the ICD-10 (i.e. H54.2), or more. Based on Snellen visual acuity, this means a cut-off point of $<0.3(20 / 60)$ for the better eye. This cut-off is related to substantial loss of QoL (i.e. utilities <0.6). ${ }^{12,13}$ A visual acuity $<0.5(20 / 40)$ of the better eye was used too as a more liberal cut-off point for end-of-life visual impairment. When analyzing visual acuities, Snellen fraction notations were converted to $\log$ MAR values using conversion tables. ${ }^{14}$ After the calculations, logMAR values were converted back to Snellen values.

We also included visual field loss in the definition of visual impairment, because, compared to visual acuity, visual fields are affected in earlier stages of glaucoma. Moreover, the number of patients with impairment from glaucoma is greatly underestimated 
when the evaluation is based solely on visual acuity. ${ }^{15}$ Various studies have shown a linear relationship between visual field status and vision-targeted QoL, even present at early disease stages. ${ }^{16,17}$ We chose an MD cut-off point of <-15 dB for the better eye to define end-of-life visual impairment. Rein and co-workers estimated that a Snellen visual acuity of 20/70 resulted in the same loss in QoL as an MD value of $-17 \mathrm{~dB} .{ }^{18}$ The ICD-10 only uses visual fields in the definition of blindness. Patients with a visual field of the better eye not greater than $10^{\circ}$ in radius around central fixation are defined as blind according to this definition. ${ }^{11}$ In order to enable comparisons with other study populations, we also applied this definition for end-of-life visual impairment in our study. When there were test points with very significant loss $(p<0.005)$ on the pattern deviation probability plot within $10^{\circ}$ around central fixation of the best eye visual field, we defined patients as visually impaired according to this definition. In case only screening HFA $(n=1)$ or Peritest visual fields $(n=5)$ were available, visual impairment was defined as at least one test point with an absolute defect within the central $10^{\circ}$ in radius around central fixation (i.e. the WHO definition). Visual impairment in patients with only unreliable visual fields ( $n=2$ ) was clinically judged by two authors (MJWB and PJGE) using all other available clinical data too.

The cause of end-of-life visual impairment was considered to be the first ophthalmic disorder which permanently reduced visual acuity or the MD value. If glaucomatous damage was judged to be the cause, we subsequently analyzed whether there was already substantial visual loss at the initial visit. This was defined as a visual field equal to an MD value $<-10 \mathrm{~dB}$ of the better eye and/or a Snellen visual acuity $<0.5(20 / 40)$ of the better eye. Substantial visual loss at the initial visit could indicate a delayed presentation of glaucoma to the ophthalmologist.

\section{Results}

\section{Population characteristics}

Descriptive statistics of the 122 included patients are described in Table 1. The mean age at death was 79.6 and 84.0 years for the men and women in our study population respectively. The mean follow-up period was 9.2 years. No patients were lost to followup due to referral to another hospital or academic centre. Seventy-three percent of all patients had their last visit in the year before they died. One hundred fifteen patients (94\%) were treated with glaucoma medication and five patients (4\%) underwent filtration surgery (Table1). We divided the population into patients initially diagnosed as having OAG (i.e. group $1, n=61$ ) and patients initially suspect of having glaucoma or had OHT (i.e. group 2, $n=61$ ). 
Table 1: Descriptive statistics of the study population

\begin{tabular}{|c|c|c|}
\hline Descriptive variables & $\begin{array}{l}\text { Open-angle glaucoma } \\
(n=61)\end{array}$ & $\begin{array}{l}\text { OHT or glaucoma suspect } \\
(n=61)\end{array}$ \\
\hline \multicolumn{3}{|l|}{ General patient characteristics } \\
\hline Female sex & $29(48 \%)$ & $32(53 \%)$ \\
\hline Age at first visit (years) & $73.8( \pm 10.0)$ & $69.5( \pm 11.2)$ \\
\hline Age at death (years) & $82.9( \pm 8.4)$ & $80.7( \pm 9.6)$ \\
\hline Follow-up period (years) & $8.3( \pm 7.0)$ & $10.1( \pm 7.2)$ \\
\hline Time between last visit and death (years) & $0.8( \pm 0.9)$ & $1.0( \pm 1.0)$ \\
\hline \multicolumn{3}{|l|}{ Treatment } \\
\hline Topical glaucoma medication during life & $59(97 \%)$ & $56(92 \%)$ \\
\hline Cataract extraction during life & $36(59 \%)$ & $36(59 \%)$ \\
\hline Laser trabeculoplasty performed during life & $15(25 \%)$ & $7(12 \%)$ \\
\hline Trabeculectomy performed during life & $5(8 \%)$ & $0(0 \%)$ \\
\hline \multicolumn{3}{|l|}{ Visual field } \\
\hline Patients with VFs available & $55(90 \%)$ & $42(69 \%)$ \\
\hline Time between last VF and death (years) ${ }^{a}$ & $3.9( \pm 3.4)$ & $6.5( \pm 3.4)$ \\
\hline Patients with reliable standard VFs available & $51(84 \%)$ & $38(62 \%)$ \\
\hline End $M D$ value of better eye $(d B)^{b}$ & $-6.9( \pm 6.4)$ & $-2.1( \pm 2.8)$ \\
\hline End MD value of worse eye $(\mathrm{dB})^{\mathrm{c}}$ & $-14.9( \pm 8.6)$ & $-5.1( \pm 6.1)$ \\
\hline \multicolumn{3}{|l|}{ Visual acuity } \\
\hline Patients with VA available & $61(100 \%)$ & $61(100 \%)$ \\
\hline Time between last VA and death (years) & $1.3( \pm 1.1)$ & $1.3( \pm 1.1)$ \\
\hline Starting VA of better eye (Snellen) & $0.64( \pm 0.23)$ & $0.73( \pm 0.23)$ \\
\hline Starting VA of worse eye (Snellen) ${ }^{d}$ & $0.28( \pm 0.44)$ & $0.51( \pm 0.32)$ \\
\hline End VA of better eye (Snellen) & $0.47( \pm 0.40)$ & $0.61( \pm 0.28)$ \\
\hline End VA of worse eye (Snellen) ${ }^{d}$ & $0.11( \pm 0.22)$ & $0.36( \pm 0.36)$ \\
\hline
\end{tabular}

Values are displayed as means and standard deviations or as frequencies and percentages.

${ }^{a}$ For the 97 patients with visual fields available.

${ }^{\mathrm{b}}$ For the 89 patients with available reliable standard 24-2 or 30-2 Humphrey visual fields.

${ }^{c}$ For the 80 patients with bilateral reliable standard 24-2 or 30-2 Humphrey visual fields available.

${ }^{d}$ Excluding two totally blind eyes at the start of the follow-up period and three at the last visit.

$\mathrm{OHT}=$ ocular hypertension, $\mathrm{VF}=$ visual field, $\mathrm{VA}=$ visual acuity, $\mathrm{MD}=$ Mean Deviation, $\mathrm{dB}=$ decibels .

Visual fields were available from 55 OAG patients (90\%) and 42 OHT patients or glaucoma suspects (69\%). No visual fields were obtained from the remaining 25 patients due to various reasons. Advanced patient's age and a short follow-up period were the main reasons for not performing visual field tests. Patients with at least one visual field available had a mean initial age of 70.4 years and a mean follow-up period of 10.7 years, compared with 76.6 years and 3.3 years in patients without visual fields respectively. 
Eighty-nine patients had reliable standard HFA visual fields available. Because the mean number of reliable standard visual fields per eye was 2.6, a rate of visual field progression could not be estimated reliably. All 122 patients had visual acuity available for both eyes. Two eyes of two patients were totally blind at the start and three eyes of three patients were totally blind (i.e. with ot without light perception) at the end of the follow-up period. None of them were caused by glaucoma.

\section{Visual impairment}

Sixteen OAG patients (26\%) and five patients with OHT or suspect for glaucoma (8\%) had an end-of-life visual impairment. All 21 visually impaired cases were confirmed by studying their patient records. Moreover, 11 of the 21 patients were referred to a multidisciplinary low vision rehabilitation centre to help them cope with their visual impairment.

Eight (15\%) out of all 55 OAG patients with visual fields available had an end-of-life visual impairment due to visual field loss (Table 2A). In two of these patients, visual impairment was based on screening or Peritest visual fields (i.e. patients 2 and 3 in Table 2A). None of the 42 patients with fields available in group 2 had visual impairment due to visual field loss. Using the WHO criterion of visual loss within $10^{\circ}$ in radius around central fixation, 29 (53\%) out of 55 patients in group 1 and 8 (19\%) out of 42 patients in group 2 were visually impaired based on visual fields at the end of life. All impaired patients based on the MD criterion were also impaired based on the WHO criterion.

Ten (16\%) out of all OAG patients had an end-of-life visual impairment due to loss in visual acuity (Table 2B). Two of them were also visually impaired based on visual fields (i.e. patients 1 and 2 in Table 2A and Table 2B). Five (8\%) patients in group 2 were impaired based on visual acuity. Using the more liberal cut-off point of an end-of-life visual acuity $<0.5(20 / 40)$ of the better eye, we found 17 patients $(28 \%)$ and 8 patients (13\%) with a visual impairment based on visual acuity in groups 1 and 2 respectively. The distribution of end-of-life MD and visual acuity values of the better eyes is shown for both diagnostic groups in Fig. 2.

\section{Explanations of visual impairment}

The individual explanations of end-of-life visual impairment are shown in the last column of Table 2. Among the OAG patients, nine (15\%) impaired cases were caused by glaucomatous disease (Table 2). Eight of them had substantial visual loss at the initial visit. Seven of these cases also had glaucoma-related visual complaints at the initial visit. Six (10\%) impaired OAG cases were mainly explained by ocular comorbidity. Comorbidi- 
ty and glaucoma contributed equally to the visual impairment in one case (i.e. case 10 in Table 2B). All impaired cases in group 2 were explained by ocular comorbidity, mainly age-related macular degeneration (AMD). Overall, all end-of-life visual impairments based on visual fields were explained by glaucomatous disease (Table 2A). When based on visual acuity, only three out of 15 impaired cases were explained by glaucoma (Table 2B).
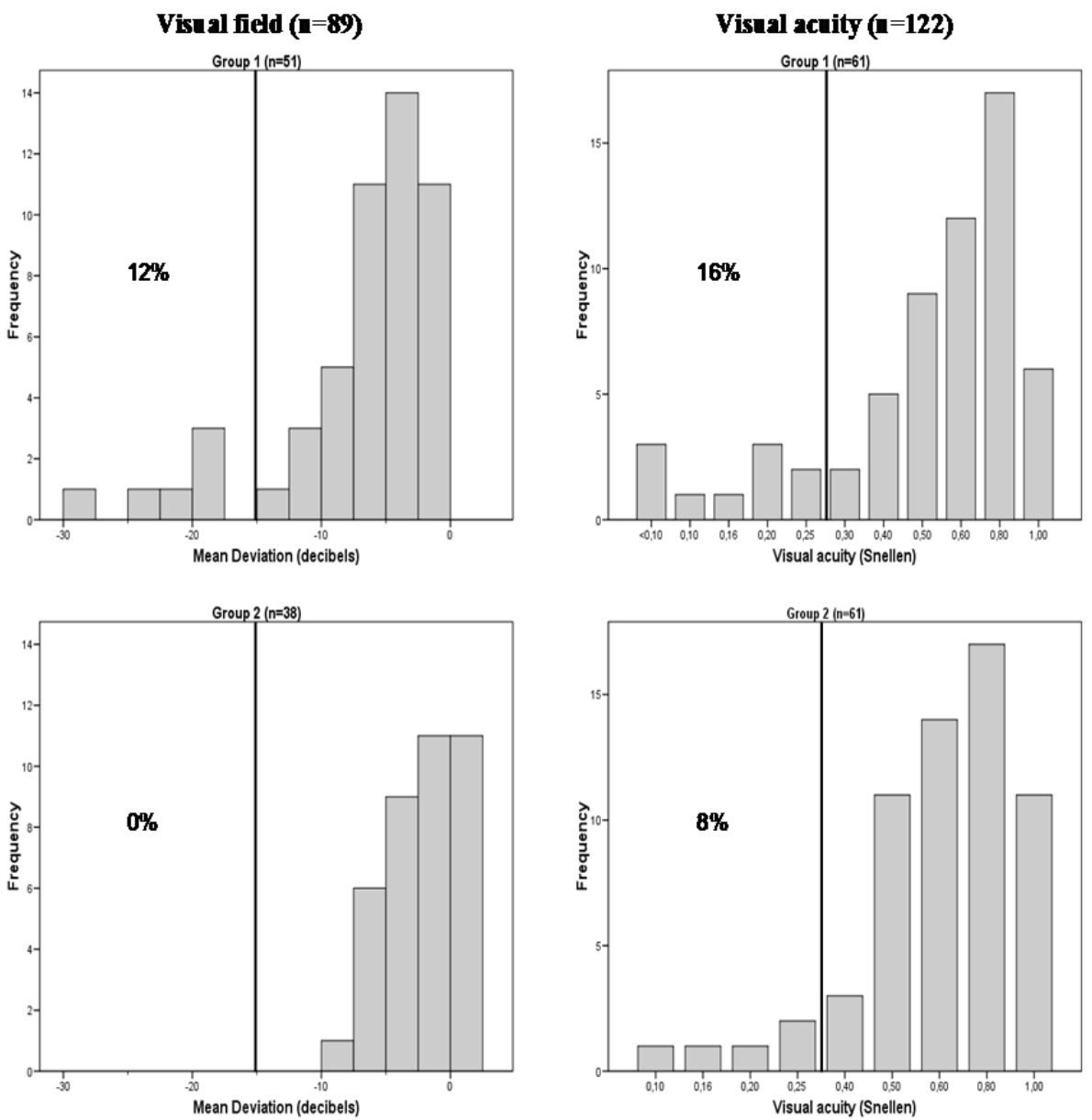

Figure 2 Histograms of the visual fields and visual acuities of the better eye before death

The vertical lines indicate the cut-off points which are used in this study to define visual impairment. The percentage of patients in each diagnostic group falling beneath this cut-off point is shown in the histograms. Two additional patients, who are not shown in this figure, had an end-of-life visual impairment based on screening or Peritest visual fields.

Group 1 = open-angle glaucoma, group 2 = ocular hypertension or glaucoma suspect. 


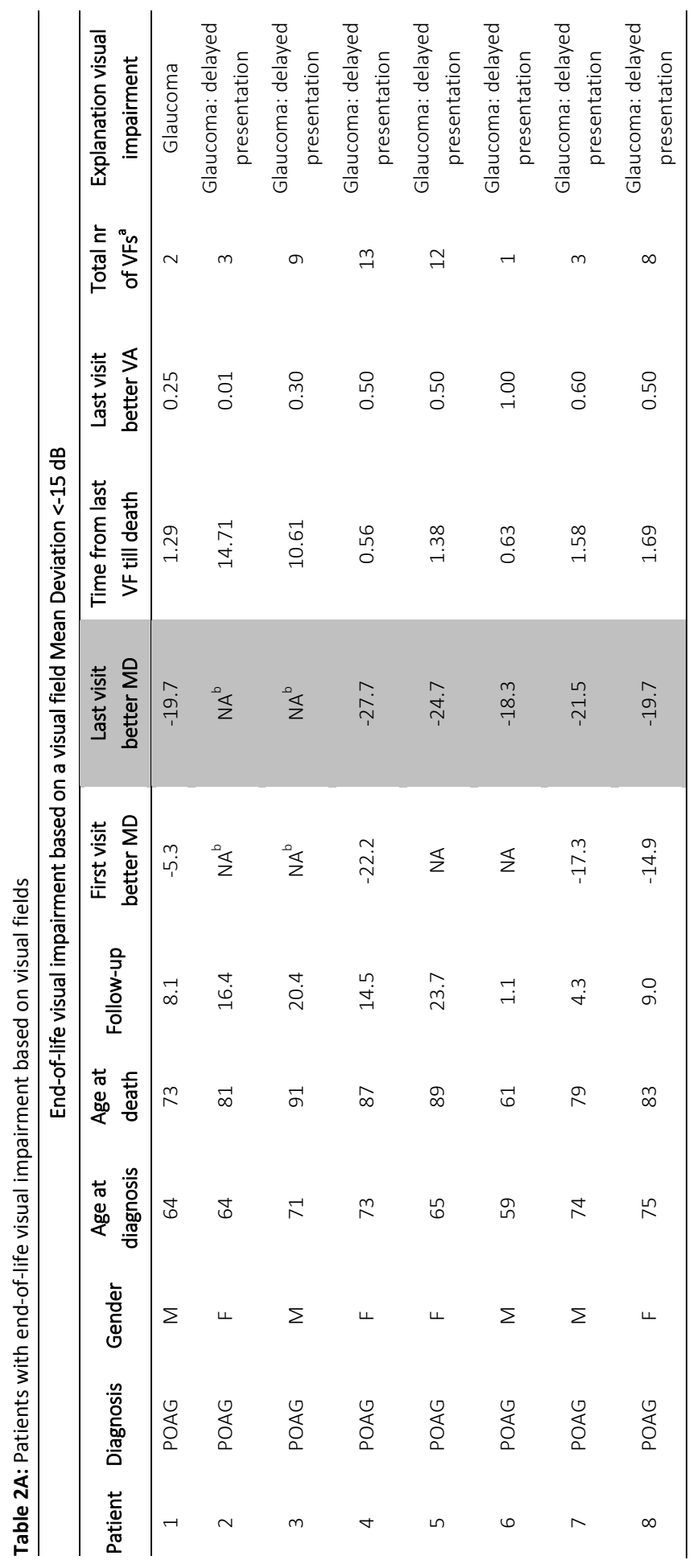




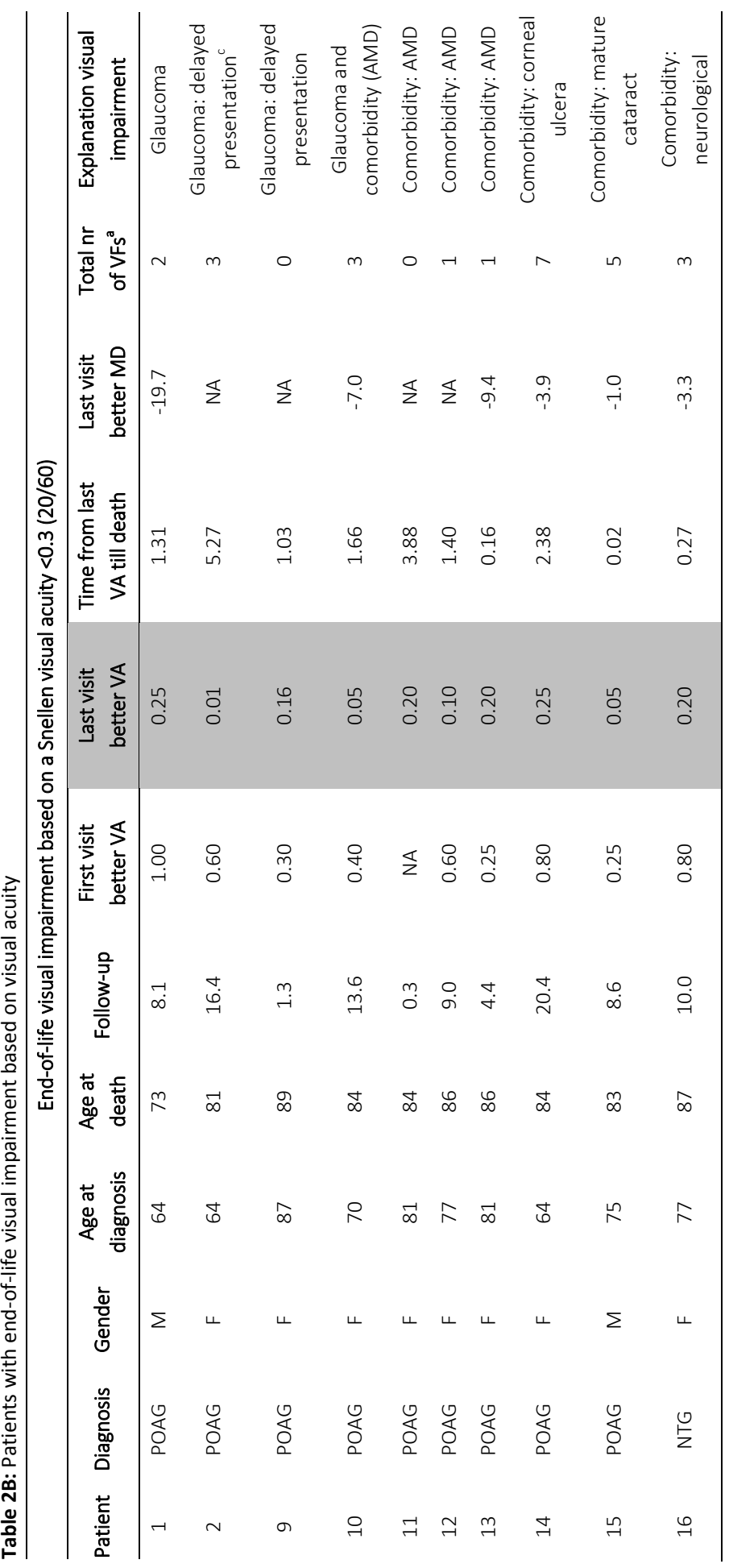




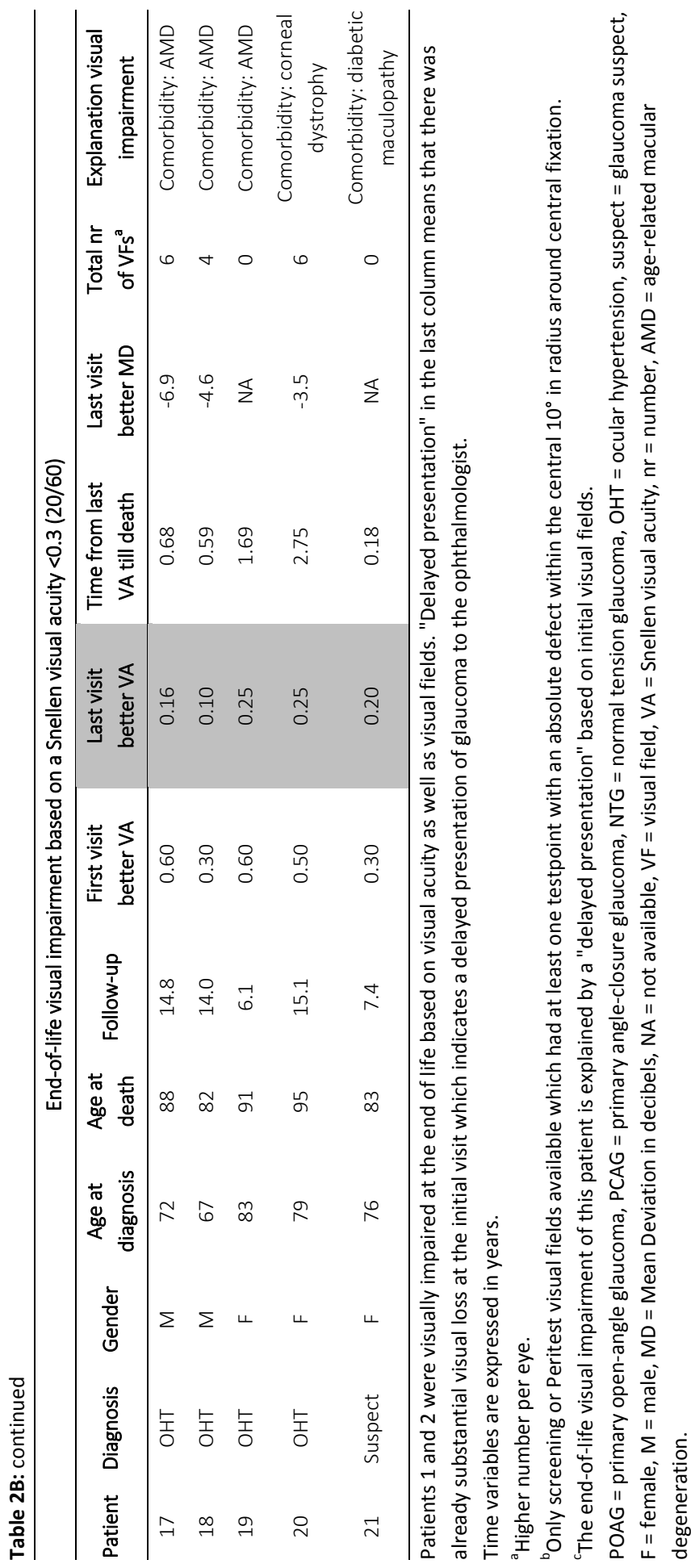




\section{Discussion}

Few studies previously investigated end-of-life visual impairment in glaucoma patients. ${ }^{19,} 20$ Forsman and colleagues studied 66 OAG patients and 40 OHT patients who had died between 1991 and 2002 of which sixteen percent were visually impaired at the last visit. ${ }^{19}$ However, visual field results were not used in their definition of visual impairment, which was defined as a Snellen visual acuity $<20 / 60$ of the better eye. ${ }^{19}$ Since we found that visual fields were the most sensitive in tracing glaucoma-related visual impairment, Forsman and co-workers are likely to have underestimated the number of patients with visual impairment. The higher prevalence of exfoliation syndrome $(42 \%)$ in their study may be one of the explaining factors for their higher true percentage of end-of-life visual impairment. ${ }^{19}$ In another study, end-of-life visual function of OAG patients was defined by binocular criteria derived from driving license criteria (i.e. Snellen visual acuity $\leq 20 / 40$ and no significant loss within the central visual field) and partial sight certifications (i.e. Snellen visual acuity $<20 / 200$, or $<20 / 60$ with a gross field defect). ${ }^{20}$ Based on these definitions, $18.2 \%$ and $6.6 \%$ of patients had an endof-life visual impairment caused by glaucoma alone, respectively. The $15 \%$ of OAG patients with end-of-life visual impairment caused by glaucoma in our study lies between their more liberal and more conservative criteria of visual impairment. ${ }^{20}$

Most cases of end-of-life visual impairments in this study were caused by ocular comorbidity. Not surprisingly, AMD was found in this study as the most important ocular comorbidity causing a visual acuity $<0.3(20 / 60)$. Using a mathematical model, a recent study estimated AMD as the most important cause of a visual acuity $<20 / 60$ of the better eye in the Dutch elderly in 2008. ${ }^{21}$ Glaucomatous disease followed on the sixth place. The prevalence of visual impairment for persons above 50 years from Western descent in the Netherlands was estimated in the same paper at $3.8 \%{ }^{21}$ We found a much higher lifetime prevalence of visual impairment, because our population had an older age and all patients visited the ophthalmologist for ocular disease. We also judged visual impairment based on visual fields which may have resulted in extra visually impaired cases.

This study shows that the eventual risk for visual impairment is considerable for patients followed for glaucoma. Most patients with an end-of-life visual impairment caused by glaucoma had already substantial visual loss at the initial visit. This indicates a delayed presentation of glaucoma to the ophthalmologist with a subsequent delay in glaucoma treatment. Evaluation of the last visual field before death detected more cases of impairment due to glaucoma than visual acuity. From 25 patients, no visual fields were available. We found that these patients were older than patients with available visual 
fields. Older age is one of the most important factors that is associated with conversion to glaucoma and glaucoma progression. ${ }^{22}$ For these reasons, the current estimation of visually impaired patients is a lower limit. 


\section{References}

1. European Glaucoma Society. Terminology and Guidelines for Glaucoma (3rd ed.). Savona, Italy: Dogma, 2008.

2. Topouzis F, Coleman AL, Harris A, Koskosas A, Founti P, Gong G, et al. Factors associated with undiagnosed open-angle glaucoma: the Thessaloniki Eye Study. Am J Ophthalmol 2008;145:327-35.

3. Wong EY, Keeffe JE, Rait JL, Vu HT, Le A, McCarty Ph DC, et al. Detection of undiagnosed glaucoma by eye health professionals. Ophthalmology 2004;111:1508-14.

4. Hatt S, Wormald R, Burr J. Screening for prevention of optic nerve damage due to chronic open angle glaucoma. Cochrane Database Syst Rev 2006:Cd006129.

5. Stoutenbeek R, Jansonius NM. Glaucoma screening during regular optician visits: can the population at risk of developing glaucoma be reached? Br J Ophthalmol 2006;90:1242-4.

6. Oliver JE, Hattenhauer MG, Herman D, Hodge DO, Kennedy R, Fang Yen M, et al. Blindness and glaucoma: a comparison of patients progressing to blindness from glaucoma with patients maintaining vision. Am J Ophthalmol 2002;133:764-72.

7. Rossetti L, Goni F, Denis P, Bengtsson B, Martinez A, Heijl A. Focusing on glaucoma progression and the clinical importance of progression rate measurement: a review. Eye 2010;24 Suppl 1:S1-7.

8. Centraal Bureau voor de Statistiek. Onverzekerden; tegen ziektekosten naar leeftijd en geslacht. Available at: http://statline.cbs.nl/StatWeb/publication/?VW=T\&DM=SLNL\&PA=71433ned\&D1=a\&D2=0\&D3=a\&$\mathrm{D} 4=\mathrm{a} \& H D=080513-1429 \& H D R=T$ GS, . Accessed May 12, 2011.

9. Kobelt G, Jonsson B, Bergstrom A, Chen E, Linden C, Alm A. Cost-effectiveness analysis in glaucoma: what drives utility? Results from a pilot study in Sweden. Acta Ophthalmol Scand 2006;84:363-71.

10. van Gestel A, Webers CA, Beckers HJ, van Dongen MC, Severens JL, Hendrikse F, et al. The relationship between visual field loss in glaucoma and health-related quality-of-life. Eye 2010;24:1759-69.

11. World Health Organization. International Classification of Diseases. Available at: http://apps.who.int/classifications/apps/icd/icd10online/index.htm?gh53.htm. Accessed April 7, 2012.

12. Sharma S, Brown GC, Brown MM, Shah GK, Snow K, Brown H, et al. Converting visual acuity to utilities. Can J Ophthalmol 2000;35:267-72.

13. Brown GC, Sharma S, Brown MM, Kistler J. Utility values and age-related macular degeneration. Arch Ophthalmol 2000;118:47-51.

14. Ferris FL, 3rd, Kassoff A, Bresnick GH, Bailey I. New visual acuity charts for clinical research. Am J Ophthalmol 1982;94:91-6.

15. Heijl A, Aspberg J, Bengtsson B. The effect of different criteria on the number of patients blind from openangle glaucoma. BMC Ophthalmol 2011;11:31.

16. Gutierrez P, Wilson MR, Johnson C, Gordon M, Cioffi GA, Ritch R, et al. Influence of glaucomatous visual field loss on health-related quality of life. Arch Ophthalmol 1997;115:777-84.

17. McKean Cowdin R, Wang Y, Wu J, Azen SP, Varma R. Impact of visual field loss on health-related quality of life in glaucoma: the Los Angeles Latino Eye Study. Ophthalmology 2008;115:941-8.

18. Rein DB, Wirth KE, Johnson CA, Lee PP. Estimating quality-adjusted life year losses associated with visual field deficits using methodological approaches. Ophthalmic Epidemiol 2007;14:258-64.

19. Forsman E, Kivela T, Vesti E. Lifetime visual disability in open-angle glaucoma and ocular hypertension. J Glaucoma 2007;16:313-9.

20. Ang GS, Eke T. Lifetime visual prognosis for patients with primary open-angle glaucoma. Eye 2007;21:6048.

21. Limburg H, Keunen JE. Blindness and low vision in The Netherlands from 2000 to 2020-modeling as a tool for focused intervention. Ophthalmic Epidemiol 2009;16:362-9.

22. Coleman AL, Miglior S. Risk factors for glaucoma onset and progression. Surv Ophthalmol 2008;53 Suppl1:S3-10. 

CHAPTER 8

Summarizing discussion 


\section{Context of glaucomatous visual field progression}

The central topic of the research presented in this doctoral thesis is visual field progression in patients with open-angle glaucoma (OAG). OAG is a lifelong disease with usually slow visual field progression over months to years. ${ }^{1}$ The goal of glaucoma treatment is to maintain the patient's visual function and related quality-of-life, at a sustainable cost. $^{2}$ Cure of lost nerve fibers is not possible. The treatment of OAG patients (i.e. lowering of intraocular pressure (IOP)) appears to be cost-effective compared with no treatment. $^{3}$

Treatment decisions should be made with regard to the patient's individual characteristics, taking into account baseline stage of disease and the (expected) rate of disease progression in the light of age-adjusted life expectancy. ${ }^{4}$ Clinical judgment leads to the decision to initiate (aggressive) treatment (i.e. mostly lowering of IOP) when current disease stage is advanced, a high disease progression is expected, and/or when life expectancy is long. In contrast, the decision to refrain from treatment, or certainly not treat aggressively, is made when current disease stage is only mild, predicted disease progression is low, and/or life expectancy is short. Personalized care is the result of the best balance between benefits and adverse events of treatment and monitoring. Recent research has shown that especially the rate of disease progression and the duration of life are of importance in making cost-effective treatment decisions for individual patients (i.e. determinants of the expected value of individualized care). ${ }^{5}$

Assessment of visual field progression is thus a central aspect of glaucoma management. The ability to identify rapidly progressing patients enables ophthalmologists to direct more intensive treatment to those who are at a high risk of becoming blind, and to refrain from intensifying potentially harmful treatment in those who have no progression. In clinical practice this can be achieved either by predicting progression or by monitoring progression. This thesis is focused on both approaches.

\section{Prediction of glaucomatous visual field progression}

If a patient is diagnosed with glaucoma, it has to be decided whether to treat, how to treat and how intense to treat. The prognosis of a patient forms the point of departure for these aspects of patient management. ${ }^{6}$ Prediction is focused on visual field progression in glaucoma management, since it gives the opportunity to intervene at an earlier stage instead of a wait-and-see strategy, thus preventing further visual field loss. Furthermore, intensive treatment can thus be avoided in patients who are unlikely to 
progress. We therefore wanted to know if and how progression can be predicted. We summarized the results from 85 studies about factors that predict glaucomatous visual field progression and ranked all factors according to their likelihood of being a prognostic factor in Chapter 2. All studied factors were classified as definite, possible and probable prognostic factors with use of a semi-quantitative approach by masked assessors. Based on the results, we advise to use the following eight factors when making decisions about treatment and monitoring strategies in glaucoma patients: age, disc hemorrhages (only for the subgroup of patients with normal-tension glaucoma (NTG)), baseline visual field loss, baseline IOP, exfoliation syndrome, central corneal thickness, peripapillary atrophy (only for the subgroup of patients with NTG), and proven previous visual field progression.

Knowing the most important prognostic factors, it would be interesting to know how accurately glaucomatous visual field progression can be predicted. Only a few prognostic models for glaucomatous visual field progression have been described. ${ }^{7,8}$ We tested the predictive ability of a combination of prognostic factors in our own population of patients with OAG in Chapter 3. A clinical prediction model was developed to predict the rate of visual field progression with the use of baseline age, IOP, and visual field status. The accuracy of a prediction model is described as explained variance (i.e. $R^{2}$ ) or as the area under the receiver operating characteristic (ROC) curve (AUC). ${ }^{9}$ During model development the $R^{2}$ of our prediction model was $10.3 \%$, which decreased to $5.3 \%$ at external validation. Our model and those of others have a mediocre accuracy in predicting an exact rate of visual field progression for individual patients. For example, the $R^{2}$ statistic of the model of De Moraes and co-workers was $14 \%$ at external validation. $^{7}$ As can be seen from their calibration plot and the calibration plots of our prediction model in Chapter 3, only a small amount of the variance in visual field progression can be explained by these models. ${ }^{10}$

This approach may not be appropriate to improve glaucoma care. Although a prediction model does not give a high $R^{2}$, it may still be possible to distinguish two or more groups in which monitoring frequency and treatment intensity can be differentiated with more benefits than universally monitoring and/or treating all patients.

The AUC of the model of De Moraes at external validation was $0.78 .^{7}$ In comparison, the AUC of our prediction model was 0.76 when the model was used to detect a VFI rate $\leq-$ $3 \%$ per year, which decreased to 0.71 at external validation. In clinical terms, the pretest probability of a high rate of visual field progression was $22.5 \%$ (i.e. the prevalence) in the population described in Chapter 3. This increased to $61 \%$ after a positive result of our prediction model and decreased to $19 \%$ after a negative test result, respectively. 
Hence, a positive result of our prediction model gives a clinical significant change in a patient's risk of future rapid progression. Prediction of progression may thus have additional value in identifying subgroups of patients with high and low rates of visual field progression. Groups of rapidly progressing patients who are detected by using the model should thus receive more intensive treatment compared with standard treatment regimes. For example, their target IOP could be estimated at lower levels than would usually have been done.

\section{Monitoring of glaucomatous visual field progression}

For the second approach to identify rapidly progressing glaucoma patients (i.e. monitoring progression), it was firstly studied how the chosen method to assess glaucomatous visual field progression influences the incidence of visual progression in patients diagnosed with OAG in the literature (see Chapter 4). This has direct consequences for the number of patients that need to be treated more intensively. We used a relatively new statistical approach (i.e. a network meta-analysis) with which it is possible to combine direct and indirect comparisons of methods from different studies to study the influence of a method on the incidence of progression. Corrected for baseline visual field loss and mean follow-up time, the mean incidence of progression of the 30 methods was $21 \%$ in six years. This ranged from $2 \%$ to $62 \%$, depending on the method used to assess progression. The chosen method accounted for nearly all heterogeneity in the incidence of progression (i.e. 82\%), and therefore strongly determines the number of glaucoma patients that are reported to have progressive disease. As expected, the cutoff point to decide whether progression had occurred also had a strong influence on the incidence of progression.

Although the method influences the number of patients reported with progression of visual field loss, it may not be true that a method with a high incidence is better in identifying patients with true progression or to differentiate better between those with and those without progression. The incidence may be unrelated to the validity of a method. To address this issue, a systematic review was conducted to identify all the methods and to show what is known about these methods, especially its validity (Chapter 5). We found 301 different methods in the literature to monitor patients for visual field progression. We found no evidence for advising one method over all others to assess visual field progression in all circumstances. However, the evidence seems to be fair for twelve methods that have been validated to some extent: the Advanced Glaucoma Intervention Study (AGIS), Collaborative Initial Glaucoma Treatment Study, pointwise linear regression analysis (PLR), mean deviation (MD), Glaucoma Change 
Probability, Early Manifest Glaucoma Treatment, Visual Field Index (VFI), Threshold Noiseless Trend, Werner, clinical scoring system, Collaborative Normal-Tension Glaucoma Study, and subjective methods. Based on these findings, a selection from one of these methods would therefore be preferable with little need to develop new methods to assess visual field progression. A difficulty in a study on the validity of a method to assess glaucomatous progression, however, is the lack of a gold standard.

To overcome this problem, the construct validity of the methods to assess glaucomatous visual field progression was investigated for ten commonly used methods (see Chapter 6). Construct validity requires a construct known to be valid. ${ }^{11}$ For example, the relation between a certain definition of glaucomatous visual field progression and IOP can be investigated. High IOP is among the most consistent prognostic factors for progression of glaucoma and is one of the few factors that can be modified by medical or surgical treatment to slow down progression. ${ }^{12,13}$ Therefore, a method that is assumed to assess glaucomatous visual field progression should find a relation between progression and IOP. When No relation between IOP and glaucomatous progression is found, one should question the validity of the assessment method. In Chapter 6, the method that had the highest association with the combination of five well-known prognostic factors in a multivariable logistic regression model was presumed to be the most valid method to assess glaucomatous visual field progression. All methods showed some degree of construct validity, although to a different extent. Expressed as AUC, the value ranged from 0.57 to 0.72 . Three methods had higher construct validity compared with the other methods: blinded subjective assessment of visual fields by "assessor C", the AGIS method, and PLR analysis using total deviation values. Within the same method, stricter progression criteria (i.e. a higher cut-off point) resulted in a stronger association with the combination of prognostic factors. This implies that in this study stricter methods are more valid methods to assess glaucomatous visual field progression, likely because the progressive patients according to highly specific methods were more "really" progressing patients. A better construct validity can therefore not only be achieved by choosing another method but also by changing the criteria for progression.

Beside the differences between progression methods in the incidence of progression and the validity, the agreement between different methods has shown to be disappointing. ${ }^{14-16}$ In Chapter 6 we confirmed this finding by reporting a mean kappa value of 0.35 (SD \pm 0.15 ) as a measure of agreement between ten different methods to assess visual field progression.

In recent literature (Chapter 5), many studies stress the importance of determining a rate of visual field progression instead of treating glaucomatous visual field progression 
as a dichotomous event. ${ }^{17}$ This is important because the rate of progression is more suitable for personalized care, as explained above. However, most studies that were included in our reviews as described in Chapter 4 and Chapter 5 treated progression as a dichotomous event (i.e. yes or no). This can be explained by the fact that, when a rate of progression is used to decide on changing therapy in clinical practice, it has to be decided which cut-off value for the rate leads to the decision to change therapy. Therefore, even a rate method leads to a dichotomisation of the population in those who progressed and need more intense treatment and those who did not. Nevertheless, it is of clinical importance to also incorporate a rate component in a dichotomous method to assess progression. Addition of a rate of progression criterion will indicate the clinical relevance of progression. This is demonstrated in the research described in Chapter 6 , where the addition of a rate component to the MD or the VFI methods increased the AUC (i.e. a measure of the presumed validity of a method) to much higher levels.

The rate of visual field progression is highly variable among patients with OAG. ${ }^{18}$ Our empirical studies as described in Chapters 3 and 6 confirmed this finding. The rate of visual field progression according to the VFI rate ranged from $-22.10 \%$ to $16.51 \%$ per year in Chapter 3 and $-13.96 \%$ to $1.98 \%$ per year in Chapter 6. For the monitoring of patients, the high variability of progression among patients improves the possibility for progression methods to discriminate between the risk profile of patients, and thus enabling different treatment modalities. ${ }^{19}$

\section{End-of-life visual impairment in glaucoma}

Finally, does it all matter? The ultimate goal of glaucoma management is to prevent visual impairment and to preserve the patient's quality-of-life. In the last decades, glaucoma-related blindness has decreased, ${ }^{20}$ but despite current treatment modalities, the prevalence of visual impairment in patients with glaucoma at the end of their life is still considerable. We assessed the prevalence of severe end-of-life visual impairment in a small population of deceased glaucoma patients in Chapter 7. In OAG, 26\% of patients had a severe end-of-life visual impairment, which was defined as a MD value <-15 decibels or a Snellen visual acuity $<0.3(20 / 60)$ of the better eye. Fifteen percent of the OAG patients had an end-of-life visual impairment that was caused by glaucoma. When also taking other studies into account, the prevalence of end-of-life visual impairment caused by glaucoma varies from $9 \%$ to $15 \%{ }^{21-23}$

Our study also gave the opportunity to study whether disease progression was the cause of end-of-life visual disability. However, we found that an initial late presentation 
of these glaucoma patients to the ophthalmologist was the most common contributing factor for their loss of vision, and age-related macular degeneration was the second most common cause. Disease progression after diagnosis was the cause of end-of-life visual impairment in only one glaucoma patient. This patient did not visit the ophthalmologist and had not used his glaucoma medication for years. One large recent study systematically investigated prognostic factors associated with the lifetime risk of OAG blindness. IOP at diagnosis, worse visual field status at baseline and age at death were the most important risk factors for end-of-life blindness in this study. ${ }^{24}$ Similar to our study, visual field progression was not taken into account in the analysis.

Although the study described in Chapter 7 is small, its concept and results are relevant. This finding may mitigate the need to search for a perfect method to assess glaucomatous visual field progression and it shows that further improvements would be desirable for earlier detection of patients with glaucoma. Ophthalmologists are able to prevent glaucoma blindness to a large extent if the patients is diagnosed at an early stage. ${ }^{24}$ Current practice with a limited frequency of visual field examination and subjective judgement of progression seems to suffice to prevent blindness to a large extent. The need to monitor is further limited if treatment is intense from diagnosis. This gives a maximal effect when applied over an extended period of time. Monitoring progression will then still be necessary to determine the need for surgery, which has potentially more serious adverse events. This approach has earlier been quantified in a costeffectiveness model. This discrete event simulation model showed that the most costeffective strategy was to aim for an IOP below $15 \mathrm{mmHg}$ in all glaucoma patients from the moment of diagnosis as compared with waiting to intensify treatment until progression has occurred. ${ }^{25,26}$ This strategy may be at the expense of individual patients who experience adverse events of treatment despite a low risk of end-of-life visual impairment. Ultimately, personalized care should be implemented in dialogue with the individual patient.

\section{Clinical recommendations}

This thesis was focused on prediction and monitoring of progression of glaucoma. Treatment decisions to prevent severe visual field loss, glaucoma-related impairment and related loss of quality-of-life should be based on their estimated risks. This risk is determined by the baseline visual field loss, rate of visual field progression and life expectancy. The reduction of this risk is affected by the intensity of treatment and factors that modify the effect of treatment. 
Treatment needs to be started in every glaucoma patient. Its intensity depends on the risk of future severity of visual field loss. Current drug and laser treatment have few adverse events. Irrespective of the future risk it can thus be intensive. Monitoring is then reserved to determine the timing of surgery. A far as visual field monitoring is concerned, subjective assessment of progression combined with changes in VFI and MD seem to be valid enough to be used.

Summarizing, from our studies several practical advices for clinicians can be given:

- Choose one of the methods to assess glaucomatous visual field progression that have been validated in the literature and know the properties of the specific method used.

- Incorporate the rate of visual field progression into your methods to assess progression, because this information is needed to estimate the individual risk of end-of-life visual impairment from glaucoma.

- Use a combination of available prognostic factors for glaucomatous visual field progression to estimate the individual risk of accelerated visual field progression and modify follow-up frequency and treatment accordingly.

- When evaluating studies on diagnostic methods, critically evaluate the gold standard to which different methods have been compared. Search for studies that investigated the construct validity of a method when the choice of a gold standard is arbitrary.

- Prevention of end-of-life visual impairment due to glaucoma, taking into account current practice and opportunities for treatment, should focus on earlier detection of patients with glaucoma to achieve less visual impairment.

\section{Future research}

Current methods of diagnosing, monitoring and treating patients with OAG have a large impact on the prevention of glaucoma-related impairment. However, not all glaucoma blindness can currently be prevented. A further reduction in blindness could be achieved if patients with glaucoma are detected at an earlier stage. General populationbased screening has not shown to be cost-effective, whereas targeting specific high-risk groups may be more effective. The best way to achieve this goal needs to be investigated. $^{27}$

Further research on causes of glaucoma-related impairment is needed. This can be achieved by studying blind glaucoma patients or those with fast progression of the disease. A larger study of deceased glaucoma patients can give more accurate estimates of the contribution of baseline visual field loss, progression of glaucoma and ocular 
comorbidity that lead to the occurrence of blindness. Clinical variables that can be improved to prevent blindness could be identified.

In the end, visual field progression is an indirect outcome measure of the loss of retinal ganglion cells, which is the pathogenic mechanism of glaucomatous damage. The relation between visual field loss and retinal ganglion cell loss is probably nonlinear due to the logarithmic transformation of visual field sensitivities. ${ }^{28}$ This can be an explanation for baseline visual field loss to be an important prognostic factor for glaucomatous visual field progression, as we found in the studies described in Chapter 2, 3, 4 and 6. In eyes with more advanced damage, a smaller number of retinal ganglion cells losses would produce relatively larger changes in MD compared with eyes with early damage. ${ }^{28}$ Therefore, it is also important to measure structural changes in OAG patients, especially in early disease. The interest in using retinal nerve fiber layer thickness for assessing disease progression has recently been increased. Optical coherence tomography is one of the most promising tools in this respect. ${ }^{29}$ Unfortunately, agreement between structural and functional disease progression has shown to be poor. ${ }^{29,} 30$ Therefore, the structure-function debate should be fuelled with more research on the construct validity of methods to assess structural changes of the optic nerve. Both structure and function should be studied over time to assess the conditional risk of disease progression (e.g. structural loss as a prognostic factor for visual field loss). It may also reveal at what point in time progressive patients are diagnosed as such, after it had been decided at an earlier time point that they did not show progression. A randomised controlled trial to compare different monitoring strategies may further optimize clinical practice. 


\section{References}

1. Heijl A, Bengtsson B, Hyman L, Leske MC. Natural history of open-angle glaucoma. Ophthalmology 2009;116:2271-6.

2. European Glaucoma Society. Terminology and Guidelines for Glaucoma (3rd ed.). Savona, Italy: Dogma, 2008.

3. Tuulonen A. Economic considerations of the diagnosis and management for glaucoma in the developed world. Curr Opin Ophthalmol 2011;22:102-9.

4. Wesselink C, Stoutenbeek R, Jansonius NM. Incorporating life expectancy in glaucoma care. Eye (Lond) 2011;25:1575-80.

5. van Gestel A, Grutters J, Schouten J, Webers C, Beckers H, Joore M, et al. The role of the expected value of individualized care in cost-effectiveness analyses and decision making. Value Health 2012;15:13-21.

6. Grobbee DE, Hoes AW. Clinical epidemiology : principles, methods, and applications for clinical research. Sudbury, Mass.: Jones and Bartlett Publishers, 2009.

7. De Moraes CG, Sehi M, Greenfield DS, Chung YS, Ritch R, Liebmann JM. A validated risk calculator to assess risk and rate of visual field progression in treated glaucoma patients. Invest Ophthalmol Vis Sci 2012;53:2702-7.

8. Nouri Mahdavi K, Hoffman D, Gaasterland D, Caprioli J. Prediction of visual field progression in glaucoma. Invest Ophthalmol Vis Sci 2004;45:4346-51.

9. Steyerberg EW. Clinical prediction models : a practical approach to development, validation, and updating. New York, N.Y.: Springer, 2009.

10. Saunders LJ, Russell RA, Crabb DP. The coefficient of determination: what determines a useful $R(2)$ statistic? Invest Ophthalmol Vis Sci 2012;53:6830-2.

11. Streiner DL, Norman GR. Health measurement scales: a practical guide to their development and use. Oxford: Oxford University Press, 2008.

12. Bahrami H. Causal inference in primary open angle glaucoma: specific discussion on intraocular pressure. Ophthalmic Epidemiol 2006;13:283-9.

13. Heijl A, Leske MC, Bengtsson B, Hyman L, Bengtsson B, Hussein M. Reduction of intraocular pressure and glaucoma progression: results from the Early Manifest Glaucoma Trial. Archives of Ophthalmology 2002;120:1268-79.

14. Arnalich Montiel F, Casas Llera P, Munoz Negrete FJ, Rebolleda G. Performance of glaucoma progression analysis software in a glaucoma population. Graefes Arch Clin Exp Ophthalmol 2009;247:391-7.

15. Katz J, Congdon N, Friedman DS. Methodological variations in estimating apparent progressive visual field loss in clinical trials of glaucoma treatment. Arch Ophthalmol 1999;117:1137-42.

16. Nouri Mahdavi K, Hoffman D, Ralli M, Caprioli J. Comparison of methods to predict visual field progression in glaucoma. Arch Ophthalmol 2007;125:1176-81.

17. Rossetti L, Goni F, Denis P, Bengtsson B, Martinez A, Heijl A. Focusing on glaucoma progression and the clinical importance of progression rate measurement: a review. Eye 2010;24 Suppl 1:S1-7.

18. Heijl A, Buchholz P, Norrgren G, Bengtsson B. Rates of visual field progression in clinical glaucoma care. Acta Ophthalmol 2013;91:406-12.

19. Ernest PJ. Signal/noise ratios to compare tests for measuring visual field progression. Invest Ophthalmol Vis Sci 2010;51:6893; author reply -4.

20. Malihi M, Moura Filho ER, Hodge DO, Sit AJ. Long-Term Trends in Glaucoma-Related Blindness in Olmsted County, Minnesota. Ophthalmology 2014;121:134-41.

21. Forsman E, Kivela T, Vesti E. Lifetime visual disability in open-angle glaucoma and ocular hypertension. J Glaucoma 2007;16:313-9.

22. Peters D, Bengtsson B, Heijl A. Lifetime risk of blindness in open-angle glaucoma. Am J Ophthalmol 2013;156:724-30. 
23. Saunders LJ, Russell RA, Kirwan JF, McNaught Al, Crabb DP. Examining visual field loss in patients in glaucoma clinics during their predicted remaining lifetime. Invest Ophthalmol Vis Sci 2014;55:102-9.

24. Peters D, Bengtsson B, Heijl A. Factors associated with lifetime risk of open-angle glaucoma blindness. Acta Ophthalmol 2014;92:421-5.

25. van Gestel A, Severens JL, Webers CA, Beckers HJ, Jansonius NM, Schouten JS. Modeling complex treatment strategies: construction and validation of a discrete event simulation model for glaucoma. Value Health 2010;13:358-67.

26. van Gestel A, Webers CA, Severens JL, Beckers HJ, Jansonius NM, Hendrikse F, et al. The long-term outcomes of four alternative treatment strategies for primary open-angle glaucoma. Acta Ophthalmol 2012;90:20-31.

27. McManus JR, Netland PA. Screening for glaucoma: rationale and strategies. Curr Opin Ophthalmol 2013;24:144-9.

28. Medeiros FA, Zangwill LM, Bowd C, Mansouri K, Weinreb RN. The structure and function relationship in glaucoma: implications for detection of progression and measurement of rates of change. Invest Ophthalmol Vis Sci 2012;53:6939-46.

29. Xu G, Weinreb RN, Leung CK. Retinal Nerve Fiber Layer Progression in Glaucoma: A Comparison between Retinal Nerve Fiber Layer Thickness and Retardance. Ophthalmology 2013;120:2493-500.

30. Xin D, Greenstein VC, Ritch R, Liebmann JM, De Moraes CG, Hood DC. A comparison of functional and structural measures for identifying progression of glaucoma. Invest Ophthalmol Vis Sci 2011;52:519-26. 
CHAPTER 9

Valorization addendum 
Valorization is the process of creating value from knowledge, by making knowledge suitable or available for social or economic use and by making knowledge suitable for translation into competitive products, services, processes and new commercial activities.

Adding value to what has value is therefore the starting point to reflect on the valorization of the knowledge gained in this thesis, and additionally pointing out the ways to improve clinical practice for ophthalmologists but, above all, for our patients.

For those who need to care about finances, it also has economic value. It is well known that many ophthalmic interventions are cost-effective. In a recent paper by Brown and co-workers, it was estimated that taking into account all direct medical costs saved and expended, direct nonmedical costs saved and indirect medical costs saved, the returnon-investment of the treatment of glaucoma with a simple drug as timolol is $3957 \%{ }^{1}$ The yearly savings of treating glaucoma patients in a usual care scenario saves at least over 180 million Euros in the Netherlands when compared to not treating these patients. ${ }^{2,3}$ Improving glaucoma care and preventing glaucoma blindness will further increase the savings and return-on-investment.

To improve clinical practice, we have formulated five practical advices for clinicians in the Summarizing discussion of this thesis. In the Summarizing discussion we also suggest directions of future research based on the research in this thesis.

In this chapter about valorization, we further discuss the societal perspective and practical implications of the presented research. Medical management of glaucoma is based on monitoring of glaucoma patients for many years. A change in treatment is based on predicted disease progression or monitored disease progression (see Summarizing discussion).

Monitoring of glaucoma patients encompasses a large burden of the work at the outpatients' department. It is undertaken to assess the effect of treatment, adverse events of treatment, or complications of the disease. Its aim is to timely initiate or change treatment to improve the outcome of the disease. Monitoring requires answers to the questions of what to monitor, how to monitor, and how frequent to monitor. A basic assumption is that the outcome is improved when monitoring is undertaken as compared to no monitoring. However, "despite the considerable staff time and resources involved, monitoring is a surprisingly understudied area." 4 This thesis adds value to this neglected area of research. 
Monitoring is also about individualizing or even personalizing care (see also Summarizing discussion). This individualization of health care or personalized medicine is inevitable given the increase in diagnostic possibilities, prognostic factors and new interventions. Moreover, efficiency is improved if choices between management strategies are individualized. ${ }^{5}$ Such an approach fits the needed reform in health care to lower cost and improve outcome by improving quality of care. As formulated in an article in Harvard Business review: "It's time for a fundamentally new strategy. At its core is maximizing value for patients: that is, achieving the best outcomes at the lowest cost." This guiding principle has shown to account for already a great deal of current glaucoma care that saves sight as well as money. ${ }^{2}$

This thesis contributes to the societal debate on personalized medicine. It shows that the selection of subjects who need more intensive treatment to reduce their risk of glaucoma blindness based on the occurrence of progression is strongly influenced by the choice of the method to detect progression. Its implications for clinical practice and patients are enormous.

The percentage of patients needing additional treatment in glaucoma ranges from $2 \%$ to $62 \%$ depending on the method used to assess progression. This implies a wide range in the number of patients who need a change in therapy. If these are to be surgical interventions an enormous number of extra operations need to be conducted. Moreover, the choice of the criterion or cut-off point for one method strongly influences the number of patients who need a change in therapy.

Also with regard to the costs of glaucoma treatment, the aim of current health policy in the Netherlands is to promote the influence of health care insurance companies. They may request transparency of the care process comprising the number of interventions (e.g. operations), before reimbursement of the ophthalmological practice is initiated. In this context, ophthalmologists may be conservative in their decisions on the number of operations and the criteria to decide on an operation. Both need to know the impact of criteria on progression in this regard.

The situation is even worse than suggested by the wide range of $2 \%$ tot $62 \%$ that show progression. Chapter 5 in this thesis shows that there are many more methods, 301 to be exact, with which glaucomatous visual field progression can be assessed. These methods have been used in research or clinical practice despite that only 48 (16\%) have been validated to some extent. However, the most important question as to what extent the method predicts blindness is not known. Moreover, reproducibility studies 
have not been conducted; implying that one moment a patient may be classified as having progressive glaucoma and on another moment as not.

Ophthalmologists and patients are therefore left with methods to assess progression of which the consequences are not exactly known. The impact on the budget, in a time of budget constraints, is also unknown.

Monitoring is a form of screening and clinicians may not have viewed examinations of their patients in this way. This is, however, important. The criteria of Wilson and Jungner are used to justify screening in the general population. ${ }^{7}$ In clinical practice no such criteria are used. When we apply these criteria to the monitoring of glaucoma patients, all criteria are fulfilled except that there are no clear criteria to assess glaucomatous progression.

Screening should be compared with no screening. In the latter case patients are sent home and asked to return when they notice a change. In glaucoma this will lead to a considerably number of cases with advanced visual field loss, since patients do not notice any change until a late stage of the disease. Based on this thesis, monitoring should be addressed in a more systematic way in daily practice by viewing it as a form of screening.

Eight prognostic factors were found to be clearly associated with glaucomatous visual field progression. Clinicians could directly use these factors to make their own risk estimations. These factors could also serve as starting point for the development of new clinical prediction models. Chapter 3 presents a prediction model for visual field progression. This model can already be used to identify a group of patients with a high rate of future visual field progression. It can also serve as a starting point for the development of more accurate clinical prediction models. For example, such a prediction model can be put online to provide access for all ophthalmologists. It can automatically calculate the risk of future progression for an individual patient, after the values of the predictors have been filled out.

Quality of care is another societal issue that is addressed in this thesis. In this thesis an "epidemiological autopsy" is described in Chapter 7. Deceased glaucoma patients were identified and their visual field loss and visual acuity before death registered from their medical records. These data provide the risk of glaucoma patients to become severely visually impaired. Moreover, the causes of visual impairment can be identified. These can be separated in three major elements: the presence and amount of visual field loss at the start of treatment, progression of visual field loss, and life expectancy. Disease 
progression partly reflects the quality of care. It was shown, however, that a considerable number of the impaired glaucoma patients had already substantial visual loss at the initial visit. This indicates a delayed presentation of glaucoma to the ophthalmologist with a subsequent delay in glaucoma treatment. If the intraocular pressure is not lowered enough, patients are more at risk of becoming blind.

These findings have implications for those who have the opportunity to detect glaucoma at an early stage. Opticians and optometrist have the knowledge and equipment to do so. Another implication is in the assessment of the quality of care. An "epidemiological autopsy" should be obligatory for an ophthalmologist for all his deceased patients. It will reveal process characteristics that need improvement: the successive process of screening for glaucoma, diagnosis, indication for treatment, quality of treatment, and compliance with the treatment. It will also demonstrate which diseases have most impact on end-of-life visual impairment.

In summary, this thesis on monitoring of glaucoma patients contributes to the debate on personalized medicine and shows the need to improve on the criteria to decide on personalizing care. This needs to be known by ophthalmologists, patients, health care insurance companies and health policy makers. It has consequences for effectiveness and efficiency of healthcare.

Monitoring should be seen from a new perspective. It is in fact a kind of screening and ophthalmologists and other clinicians should be aware of that. Every glaucoma control patient needs to be observed from this perspective in order to improve effectiveness, efficiency and alleviate the large burden on the work at the outpatients' department.

An "epidemiological autopsy" is a must for every ophthalmologist to show his excellent results or improve his practice.

Finally, guidelines are guiding and, like other research from the University Eye Clinic Maastricht, this thesis will contribute to the new guidelines of the European Glaucoma Society. 


\section{References}

1. Brown MM, Brown GC, Lieske HB, Lieske PA. Financial return-on-investment of ophthalmic interventions: a new paradigm. Curr Opin Ophthalmol 2014;25:171-6

2. van Gestel A, Webers CA, Severens JL, Beckers HJ, Jansonius NM, Hendrikse F, et al. The long-term outcomes of four alternative treatment strategies for primary open-angle glaucoma. Acta Ophthalmol 2012;90:20-31.

3. Webers CA and Schouten JS. Hoe vaak komen gezichtsstoornissen voor? In: Volksgezondheid Toekomst Verkenning, Nationaal Kompas Volksgezondheid. Bilthoven: RIVM, 2014

4. Glasziou P, Irwig L, Mant D. Monitoring in chronic disease: a rational approach. BMJ 2005;330:644-8.

5. van Gestel A, Grutters J, Schouten J, Webers C, Beckers H, Joore M, et al. The role of the expected value of individualized care in cost-effectiveness analyses and decision making. Value Health 2012;15:13-21.

6. Porter ME, Lee TH. The Strategy That Will Fix Health Care. Harvard Business Review, October 2013. Available at: http://hbr.org/2013/10/the-strategy-that-will-fix-health-care/ar/1

7. Wilson JMG and Jungner G. Principles and practice of screening for disease. WHO, Public Health Papers, No. 34, 1968. 

CHAPTER 10

Samenvatting 
Het onderwerp van dit proefschrift is gezichtsveldprogressie bij patiënten met openkamerhoekglaucoom (hierna te noemen 'glaucoom'). Glaucoom is een levenslange, chronische oogziekte waarbij de functie van de oogzenuw in meer of mindere mate wordt aangetast.

Het gevolg hiervan is uitval van de randen van het zien, ofwel een beperking van het gezichtsveld (tunnelvisus). Hierdoor ziet iemand bijvoorbeeld een auto van opzij niet aankomen. Deze gezichtsvelduitval neemt -in de meeste gevallen langzaam- toe in de loop van het leven en is onherstelbaar. Dit wordt ook wel gezichtsveldprogressie genoemd. In ernstige gevallen kan de gezichtsvelduitval leiden tot slechtziendheid of blindheid.

Het belangrijkste doel van de behandeling van glaucoom is het voorkomen van blindheid en slechtziendheid en daarmee gerelateerd verlies van kwaliteit van leven. Tot op heden is de enige manier om glaucoom te behandelen het verlagen van de oogdruk, waardoor de gezichtsveldprogressie meestal kan worden afgeremd. Dit kan met behulp van medicatie (meestal in de vorm van oogdruppels), laserbehandelingen of operatieve ingrepen. In de praktijk krijgen vrijwel alle glaucoompatiënten een behandeling gericht op de verlaging van de oogdruk.

Hoe intensief een glaucoompatiënt behandeld moet worden, hangt af van zijn prognose. Met andere woorden, hoe snel het gezichtsveld van een glaucoompatiënt in de komende jaren achteruit zal gaan en wat de kans op uiteindelijke blindheid zal zijn. De kans op gezichtsveldprogressie bij glaucoom kan worden voorspeld met behulp van prognostische factoren. Prognostische factoren zijn specifieke kenmerken van een patiënt van wie het gezichtsveld in de toekomst sneller achteruit zal gaan. In hoofdstuk 2 wordt beschreven hoe in de literatuur 103 verschillende prognostische factoren werden onderzocht. Middels een systematisch literatuuronderzoek hebben we de belangrijkste factoren hieruit bepaald, te weten: hoge leeftijd, bloedinkjes op de rand van de oogzenuw, reeds veel gezichtsvelduitval als de diagnose gesteld wordt, hoge oogdruk, pseudo-exfoliatie (aanwezigheid van wit schilferig materiaal in het oog), centrale hoornvliesdikte, aanwezigheid van een gebied waarin het netvlies zich heeft teruggetrokken rondom de oogzenuw en veel gezichtsveldprogressie in het verleden.

In hoofdstuk 3 hebben we vervolgens onderzocht hoe gezichtsveldprogressie accuraat kan worden voorspeld. Met behulp van gegevens van een groep van 613 glaucoompatienten hebben we een rekenkundige methode ontwikkeld waarmee met leeftijd, oogdruk en mate van gezichtsvelduitval, de progressie voorspeld kan worden (een predictiemodel). Met deze methode kan voorspeld worden of een patiënt bij de snel 
progressieve groep glaucoompatiënten behoort. De voorspelling van de individuele mate van progressie bleek echter niet nauwkeurig genoeg.

Naast het voorspellen van gezichtsveldprogressie wordt er in de praktijk gemeten hoe snel die progressie daadwerkelijk is. Dit noemen we monitoren van gezichtsveldprogressie. Wanneer dan een te snelle progressie wordt vastgesteld, kan de behandeling geïntensiveerd worden. Over de manier (de methode) waarop gezichtsveldprogressie gemeten moet worden bestaat echter geen overeenstemming. In hoofdstuk 4 is voor elk van dertig verschillende methoden onderzocht hoeveel glaucoompatiënten gezichtsveldprogressie hadden. Hiervoor hebben we onderzoeken (studies) bestudeerd waarin meerdere methoden met elkaar worden vergeleken. Vervolgens zijn alle resultaten van de verschillende studies middels een rekenkundige methode (een netwerk meta-analyse) samengevoegd en met elkaar vergeleken. Het gemiddeld optreden van progressie (de incidentie) was 21 procent in zes jaar, variërend van twee procent tot 62 procent, afhankelijk van de gekozen methode. De gekozen methode voor het meten van gezichtsveldprogressie is bovendien de meest belangrijke factor die bepaalt hoeveel patiënten in een studie gezichtsveldprogressie hebben.

In hoofdstuk 5 hebben we middels een systematisch literatuuronderzoek beschreven wat bekend is over de beste methode om gezichtsveldprogressie te meten. De validiteit van een methode is de mate van waarschijnlijkheid waarmee de uitkomst van een methode overeenkomt met de werkelijkheid. Met andere woorden, is de gemeten gezichtsveldprogressie ook werkelijke progressie van glaucoom? In de literatuur zijn 301 verschillende methoden beschreven, waarvan slechts enkele zijn onderzocht op hun kwaliteit (gevalideerd). Sommige hiervan zijn meerdere keren onderzocht. Er bleek geen methode te bestaan voor het meten van progressie die duidelijk beter was dan alle andere methoden (geen gouden standaard).

Om het probleem van het ontbreken van een gouden standaard te omzeilen hebben we in hoofdstuk 6 de constructvaliditeit gebruikt om tien methoden voor het meten van gezichtsveldprogressie bij glaucoom verder te valideren. Constructvaliditeit houdt in dat de validiteit van een methode wordt getest met behulp van andere verbanden waarvan bekend is dat ze bestaan (een achterliggend concept). Een voorbeeld van een bekend verband, is het verband tussen oogdruk en gezichtsveldprogressie. Een methode die gezichtsveldprogressie meet zou theoretisch een relatie moeten hebben met de oogdruk, omdat we weten dat oogdruk een belangrijke prognostische factor is voor gezichtsveldprogressie. Met het verband tussen vijf bekende prognostische factoren en gezichtsveldprogressie in onze eigen populatie glaucoompatiënten, hebben we de constructvaliditeit van tien methoden onderzocht. We hebben hierbij aangenomen dat 
een methode meer valide is als er een sterker verband bestaat met de combinatie van deze vijf prognostische factoren. Hieruit is gebleken dat er verschillen zijn in constructvaliditeit.

Ten slotte hebben we in hoofdstuk 7 bestudeerd in welke mate, onder de huidige omstandigheden van voorspellen, monitoren en behandelen van gezichtsveldprogressie, blindheid en slechtziendheid worden voorkomen. Hiervoor hebben we een groep van 61 overleden glaucoompatiënten nader bestudeerd en bekeken hoeveel patiënten uiteindelijk gestorven zijn met een ernstige visuele beperking. Van de bestudeerde groep glaucoompatiënten, bleek 26 procent te zijn overleden met een visuele beperking, bij vijftien procent van de totale groep werd dit veroorzaakt door glaucoom. Over het algemeen lieten deze laatste patiënten zich pas voor het eerst door een oogarts onderzoeken als er al veel schade aan het gezichtsveld opgetreden was. De mate van uitval van het gezichtsveld aan het begin van het behandeltraject lijkt dus de belangrijkste verklaring te zijn voor een visuele beperking bij overlijden. Nader onderzoek in een grotere groep moet dit bevestigen. 

CHAPTER 11

Dankwoord 
Graag wil ik iedereen bedanken die heeft bijgedragen aan de totstandkoming van mijn proefschrift. Ook mijn (schoon)familie en vrienden die tijdens het promotietraject steeds met mij hebben meegeleefd, wil ik ontzettend bedanken voor hun voortdurende interesse en motiverende woorden.

Een aantal personen wil ik bij dezen graag in het bijzonder bedanken.

Op de eerste plaats natuurlijk het projectteam, bestaande uit mijn eerste promotor prof. dr. Carroll Webers en mijn copromotoren dr. Jan Schouten en dr. Henny Beckers, voor de prettige en waardevolle samenwerking. We hebben door de jaren heen zeer frequent overleg gevoerd en gedetailleerd de artikelen besproken die hebben geleid tot dit proefschrift. Onze bijeenkomsten heb ik als zeer positief en constructief ervaren. Ik ben erg dankbaar voor de begeleiding en het gegunde vertrouwen.

Mijn tweede promotor, prof. dr. Martin Prins, voor de ondersteuning van mijn onderzoek op de afdeling Epidemiologie. Ik vond het zeer nuttig dat hij als niet-oogarts een kritische blik wilde werpen op mijn onderzoek.

Het voormalig hoofd van de Universiteitskliniek voor Oogheelkunde Maastricht, prof. dr. Fred Hendrikse, die mij voor de opleiding tot oogarts heeft aangenomen en mij alle mogelijkheden heeft gegeven om mijn onderzoek uit te voeren.

Dr. Wolfgang Viechtbauer en dr. Michiel Busch voor hun grote hulp als medeauteur van een van de hoofdstukken van dit proefschrift.

De beoordelingscommissie voor het bestuderen van het manuscript: prof. dr. A.E.R.C.H. Boonen, prof. dr. J.M.P. Kleijnen, prof. dr. R.J. van Oostenbrugge, prof. dr. G.H.M.B. van Rens en Dr. L.J. van Rijn.

Het Glaucoomfonds en het Oogfonds voor hun financiële bijdrage waardoor dit proefschrift mede mogelijk is gemaakt. Ook wil ik mevrouw Greidanus van het Glaucoomfonds bedanken voor de feedback vanuit de patiëntenvereniging op ons onderzoek.

Alle niet-academische ziekenhuizen en oogartsen die hun medewerking hebben verleend aan onze dataverzameling: Catharina Ziekenhuis Eindhoven, Jeroen Bosch Ziekenhuis Den Bosch, Groene Hart Ziekenhuis Gouda en Ziekenhuis Amstelland Amstelveen. 
De Maastrichtse afdeling Epidemiologie wil ik graag danken voor de totaal andere invalshoek die ik me eigen heb kunnen maken tijdens mijn werkzame periode aldaar van medio 2009 tot medio 2011. Ook wil ik in het bijzonder de docenten van de master epidemiologie bedanken voor de inspirerende colleges. Ik heb deze masteropleiding met veel plezier doorlopen gedurende twee jaar van mijn promotie onderzoek.

De verschillende kamergenoten bij epidemiologie en oogheelkunde voor de kleine dingen van het promotie onderzoek. Ook wil ik de promovendi bij oogheelkunde bedanken voor alle gezellige koffiepauzes, lunches en borrels (na werktijd).

De staf van de Universiteitskliniek voor Oogheelkunde voor de blijken van waardering en hun interesse in mijn onderzoek. In het bijzonder ing. Astrid Hacking voor haar ondersteuning tijdens mijn promotieonderzoek en mijn opleiding tot oogarts.

De oogartsen in opleiding voor hun begrip voor mijn onderzoeksactiviteiten en de geweldige werksfeer in de huidige assistentengroep.

Harry van Montfort en Jos Slangen voor alle hulp bij computer gerelateerde zaken, Mireille van Helden voor de praktische hulp bij het uitvoeren van het onderzoek en Yvonne Leenders voor haar hulp bij het opmaken van dit proefschrift.

Het secretariaat van de Universiteitskliniek voor Oogheelkunde voor hun belangrijke ondersteuning bij de administratieve kant van mijn promotieonderzoek.

Mijn paranimfen, mijn zusje Marijke Ernest en goede vriendin (dr.) Linda Kerkmeijer, voor hun steun bij het afronden van dit proefschrift en het organiseren van de activiteiten rondom de promotieplechtigheid.

Mijn lieve ouders voor het vertrouwen en de motivatie die ik vanaf mijn vroege jeugd heb gekregen en die me tot hier hebben gebracht.

Liefste Thijs, jij bent door de jaren heen mijn grootste steun en toeverlaat geweest. Vanaf het moment dat ik solliciteerde naar deze promotieplaats en jij voor mijn baan vanuit onze studentenstad Nijmegen mee naar het Zuiden bent verhuisd, tot het meeleven met het al dan niet geaccepteerd krijgen van mijn artikelen, de vormgeving van dit proefschrift en jouw hulp bij de organisatie van de dag. 
Curriculum vitae 
Paul J.G. Ernest werd geboren op 24 september 1983 te Halsteren. Op het Roncalli te Bergen op Zoom heeft hij in 2001 zijn atheneumdiploma behaald. In 2001 begon hij met zijn studie geneeskunde aan de Radboud Universiteit Nijmegen, alwaar hij afstudeerde op 14 augustus 2007. Tijdens zijn wetenschappelijke stage deed hij onderzoek op de afdeling Oogheelkunde van het Radboud UMC, wat resulteerde in een wetenschappelijke publicatie. Tot 1 februari 2008 werkte hij als arts-assistent (niet in opleiding tot specialist) op de afdeling Chirurgie van Ziekenhuis Rivierenland in Tiel. Hierna begon hij bij de Universiteitskliniek voor Oogheelkunde Maastricht en de afdeling Epidemiologie van de Universiteit Maastricht met het promotieonderzoek waarvan de resultaten zijn beschreven in dit proefschrift. Tijdens het promotieonderzoek heeft hij op 31 januari 2011 cum laude de master opleiding "public health" (afstudeerrichting epidemiologie) aan de Universiteit Maastricht afgerond. Vanaf 1 september 2011 is hij verbonden aan de Universiteitskliniek voor Oogheelkunde Maastricht als arts-assistent in opleiding tot oogarts. Naar verwachting zal hij oogarts zijn vanaf 1 maart 2016. 
Presentaties

26 maart 2009:

Jaarvergadering Nederlands Oogheelkundig Gezelschap, Groningen, mondeling

12 juni 2009:

Werkgroep Epidemiologisch Onderzoek Nederland, Amsterdam, mondeling

6 november 2009: Nederlandse afdeling van "The Association for Research in Vision and Ophthalmology", Rotterdam, mondeling

3 maart 2010:

Refereeravond Universiteitskliniek voor Oogheelkunde Maastricht, Beek, mondeling

26 maart 2010: Jaarvergadering Nederlands Oogheelkundig Gezelschap, Maastricht, mondeling

12-17 september 2010: Congres van de "European Glaucoma Society", Madrid, poster

3 maart 2011: $\quad$ Refereren afdeling Epidemiologie, Universiteit Maastricht, Maastricht, mondeling

2 april 2011: Jaarvergadering Nederlands Oogheelkundig Gezelschap, Maastricht, mondeling

16 juni 2011: Glaucoomavond Merck Sharp \& Dohme, Heeze, mondeling

29 juni - 2 juli 2011: "World Glaucoma Congress", Parijs, poster

7 december 2011:

Refereeravond Universiteitskliniek voor Oogheelkunde Maastricht, Beek, mondeling

29 maart 2012: Jaarvergadering Nederlands Oogheelkundig Gezelschap, Groningen, mondeling

17-22 juni 2012: Congres van de "European Glaucoma Society", Kopenhagen, poster

31 oktober 2012: $\quad$ Glaucoomavond Allergan, Maastricht, mondeling

6 maart 2013: Wetenschapsmiddag Arts-Assistenten Vereniging MUMC, Maastricht, poster

21 maart 2013: Jaarvergadering Nederlands Oogheelkundig Gezelschap, Groningen, mondeling

11 april 2014: Vergadering Nederlandse Glaucoom Groep, Utrecht, mondeling 


\section{Publicaties}

Ernest P.J.G., Boon C.J., Klevering B.J., Hoefsloot L.H., Hoyng C.B. Outcome of ABCA4 microarray screening in routine clinical practice. Molecular Vision 2009;15:2841-7.

Ernest P.J.G., Viechtbauer W., Schouten J.S.A.G., Beckers H.J.M., Hendrikse F., Prins M.H., Webers C.A.B. The influence of the assessment method on the incidence of visual field progression in glaucoma: a network meta-analysis. Acta Ophthalmologica 2012;90:10-9.

Ernest P.J.G. Signal/noise ratios to compare tests for measuring visual field progression (letter). Investigative Ophthalmology and Visual Science 2010;51:6893

Ernest P.J.G., Schouten J.S.A.G., Beckers H.J.M., Hendrikse F., Prins M.H., Webers C.A.B. The evidence base to select a method for assessing glaucomatous visual field progression. Acta Ophthalmologica 2012;90:101-8.

Ernest P.J.G., Schouten J.S.A.G., Beckers H.J.M., Hendrikse F., Prins M.H., Webers C.A.B. An evidence-based review of prognostic factors for glaucomatous visual field progression. Ophthalmology 2013;120:512-9.

Ernest P.J.G., Busch M.J.W.M., Webers C.A.B., Beckers H.J.M., Hendrikse F., Prins M.H., Schouten J.S.A.G. Prevalence of end-of-life visual impairment in patients followed for glaucoma. Acta Ophthalmologica 2013;91:738-43.

Ernest P.J.G., Schouten J.S.A.G., Beckers H.J.M., Hendrikse F., Prins M.H., Webers C.A.B. Prediction of glaucomatous visual field progression using baseline clinical data. Journal of Glaucoma 2014, published ahead-of-print, doi: 10.1097/IJG.0000000000000146 
